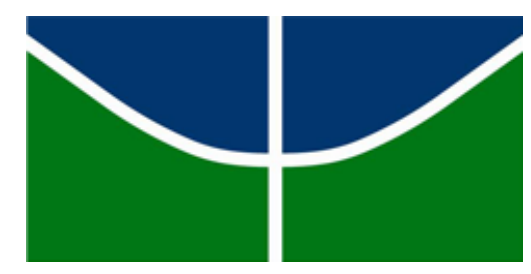

UNIVERSIDADE DE BRASÍLIA INSTITUTO DE GEOCIÊNCIAS

\title{
"ESTUDOS GEOQUÍMICOS E GEOCRONOLÓGICOS APLICADOS ÀS ROCHAS GRANÍTICAS DO GARIMPO TRAIRÃO - MT"
}

MARA LUIZA BARROS PITA ROCHA

Tese de Doutorado $\mathbf{N}^{\circ} 130$

Brasília-DF, junho de 2016 


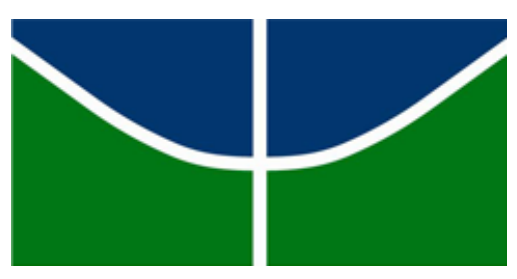

UNIVERSIDADE DE BRASÍLIA

INSTITUTO DE GEOCIÊNCIAS

\section{"ESTUDOS GEOQUÍMICOS E GEOCRONOLÓGICOS APLICADOS ÀS ROCHAS GRANÍTICAS DO GARIMPO TRAIRÃO - MT"}

TESE DE DOUTORADO

\section{Autora: MARA LUIZA BARROS PITA ROCHA}

\section{Orientador: Prof. Dr. Farid Chemale Junior}

Tese de Doutorado elaborada junto ao Programa de Pós-Graduação em Geologia (Área de Concentração Prospecção e Geologia Econômica), do Instituto de Geociências (IG) da Universidade de Brasília (UnB) para obtenção do Título de Doutor em Geologia. 


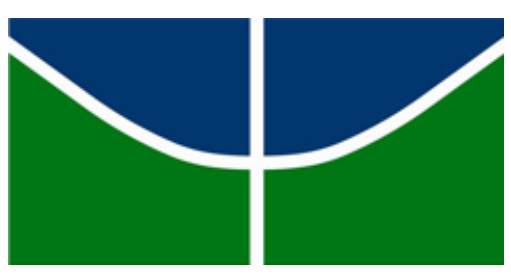

UNIVERSIDADE DE BRASÍLIA

INSTITUTO DE GEOCIÊNCIAS

\section{"ESTUDOS GEOQUÍMICOS E GEOCRONOLÓGICOS APLICADOS ÀS ROCHAS GRANÍTICAS DO GARIMPO TRAIRÃO - MT"}

MARA LUIZA BARROS PITA ROCHA

Tese de Doutorado

Orientador: Prof. Dr. FARID CHEMALE JUNIOR

Banca examinadora:

Prof. Dr. Amarildo Salina Ruiz (UFMT)

Prof. a. Dra. Márcia Abrahão Moura (UnB)

Prof. Dr. Nilson Francisquini Botelho (UnB)

Prof. Dr. Valmir da Silva Souza (UnB) 


\section{AGRADECIMENTOS}

Agradeço principalmente ao professor e orientador Dr. Farid Chemale Junior, pela enorme paciência e respeito tido comigo no decorrer dos quase quatro anos de trabalho em conjunto.

A minha família, quero agradecer pelo apoio e compreensão.

Ao meu marido Renato Costa Sala, que sempre esteve ao meu lado durante todo esse trabalho me apoiando e incentivando, inclusive nas escolhas geológicas.

A minha querida orientadora e amiga Márcia Barros, quero agradecer pelo estimulo ao desenvolvimento dessa pesquisa.

Ao professor João Orestes Schneider Santos, que me apresentou o incrível mundo da geocronologia, gostaria de agradecer também pelo auxílio durante meu período de estágio na Austrália.

Agradeço a todos os membros do grupo de pesquisa da PAAF, especialmente aos

professores da UFMT, Francisco Pinho, Paulo Cesar Corrêa da Costa, Élzio Barbosa, a amiga e companheira de longas conversas geológicas Fernanda Rodrigues da Silva.

Ao CNPQ pela concessão da Bolsa de doutorado e pelos auxílios à pesquisa concedidos tanto durante as etapas e usos de laboratórios, ao Projeto de Pesquisa PROCAD - UFMT UNICAMP, ao Instituto de Geociências da USP, a University of Western Australia e ao laboratório de geocronologia da Curtin University.

A todos os professores do Instituto de Geociências da UnB por todo o apoio e oportunidades acadêmicas, profissionais e pessoais oferecidas que contribuíram na minha formação.

Enfim, agradeço a todas as pessoas que contribuíram direta ou indiretamente na preparação dessa Tese de Doutorado. 


\section{SUMÁRIO}

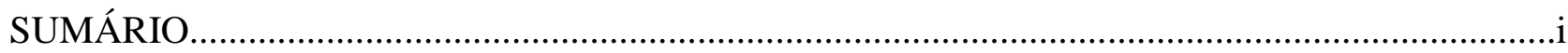

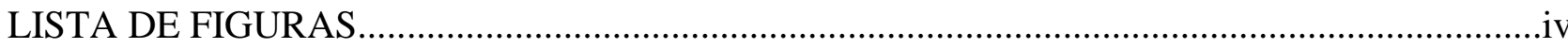

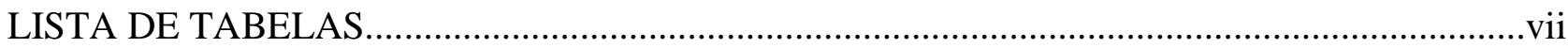

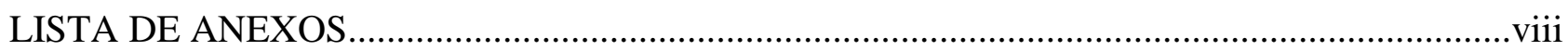

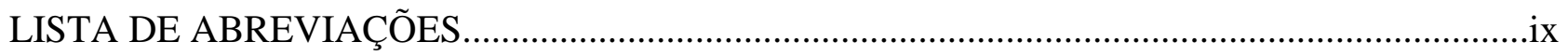

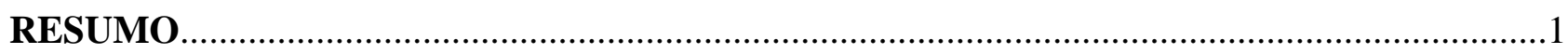

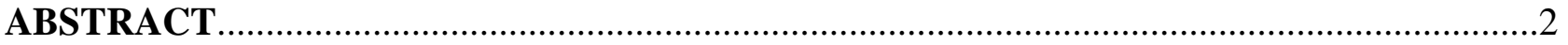

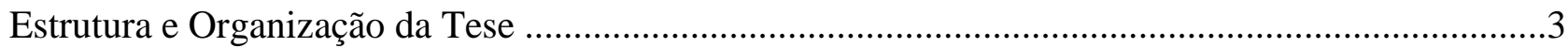

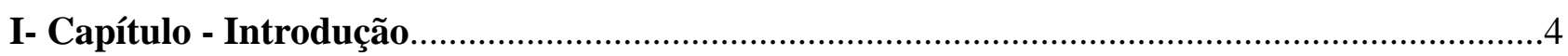

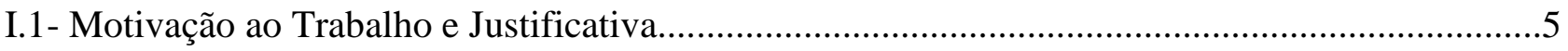

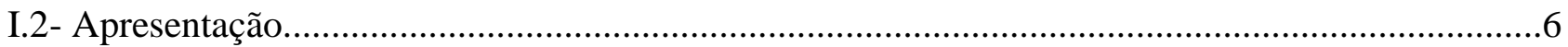

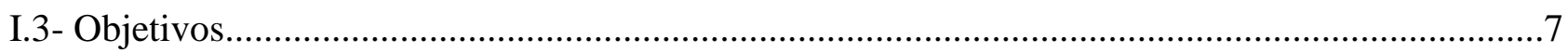

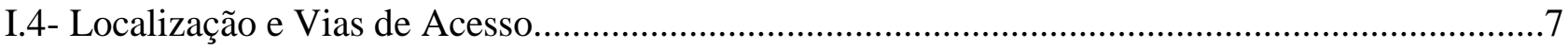

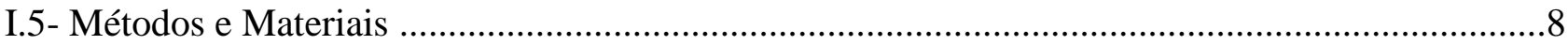

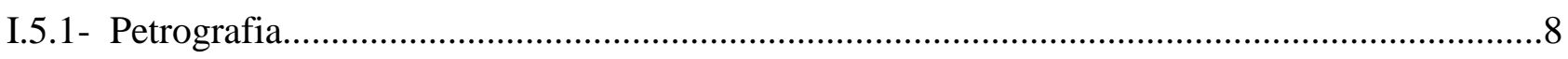

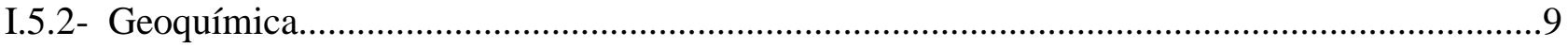

I.5.3- Microscopia Eletrônica - Química Mineral em Monazita .....................................................9

I.5.4- Microscopia Eletrônica - Química Mineral em Zircão..........................................................10

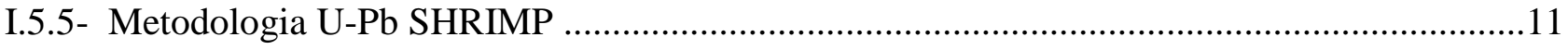

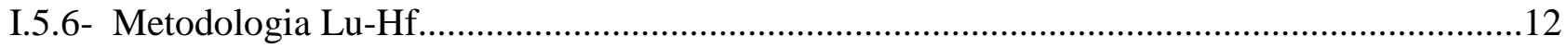

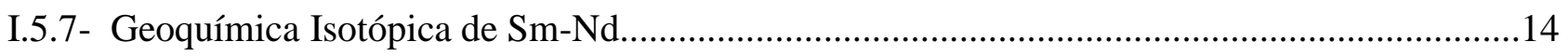

II - Capítulo - Contexto Geológico.....................................................................................

II.1- Contexto Geológico Geotectônico....................................................................................... 16

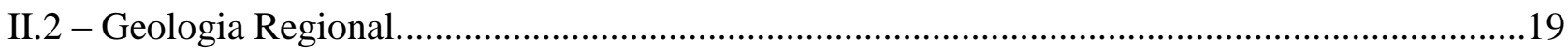

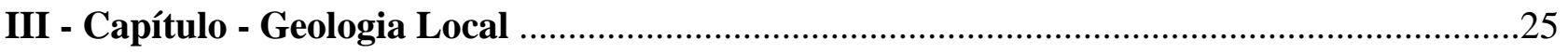

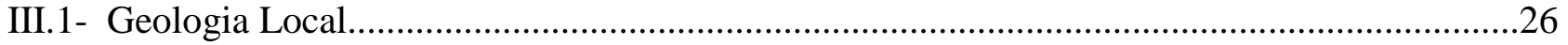

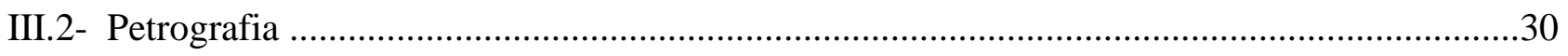

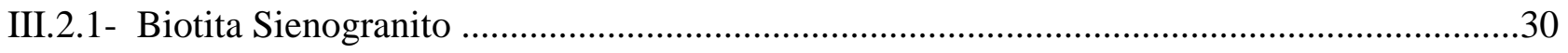

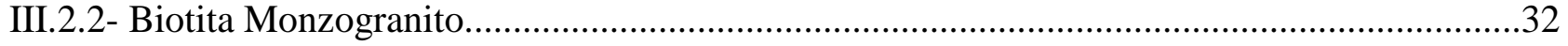

III.2.3- Enclaves microgranulares félsicos e intermediários..........................................................34

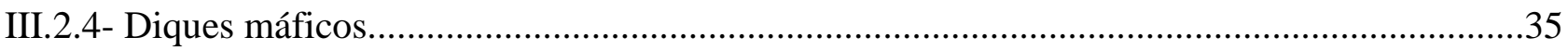

Referências da Tese 
IV."Isotope Geology of the Granitic Rocks From Trairão Region - Alta Floresta Gold Province -

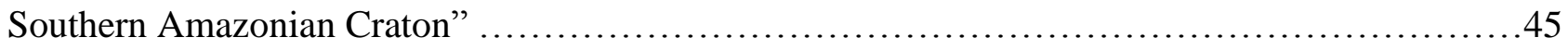

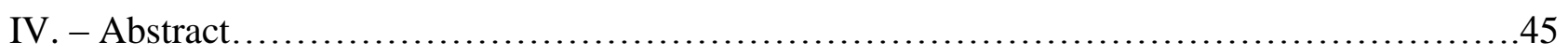

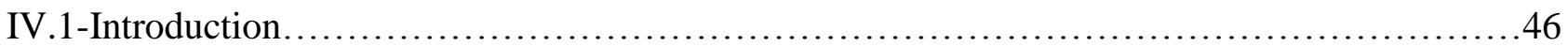

IV.2- Geotectonic Context of the Amazonian Craton.......................................46

IV.2.1- Alta Floresta Region Geotectonic Setting..........................................49

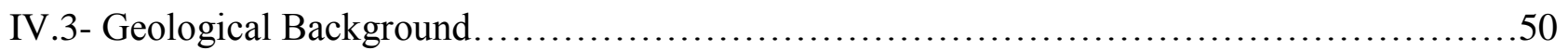

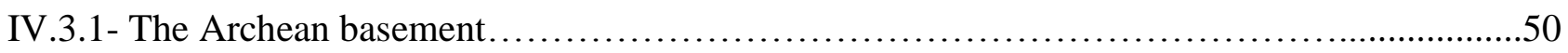

IV.3.2- Nhandu Intrusive Suite.................................................. 50

IV.3.3- Matupá Suite................................................................ 51

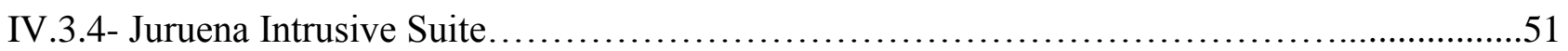

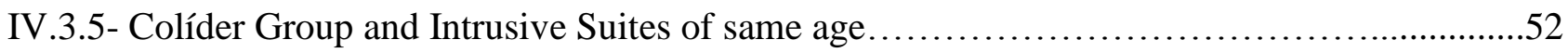

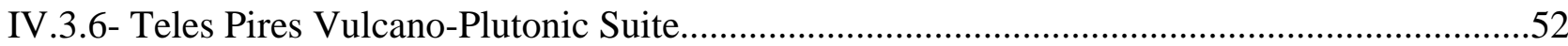

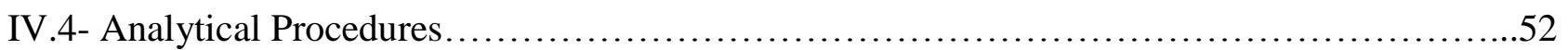

IV.4.1- Petrography and Lithogeochemistry........................................... 52

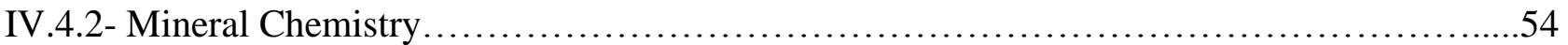

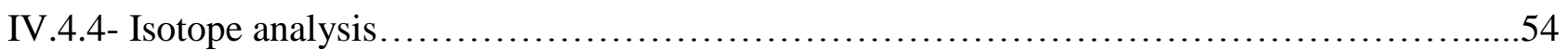

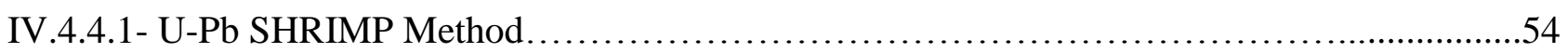

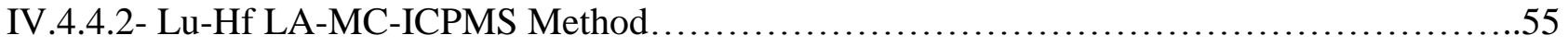

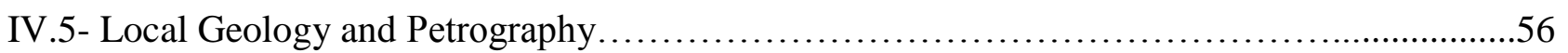

IV.5.1- Biotite Monzogranite (Nhandu Intrusive Suite) ....................................56

IV.5.2- Biotite Syenogranite (Matupá Intrusive Suite) ...................................57

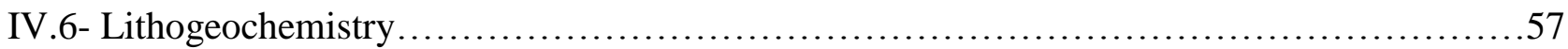

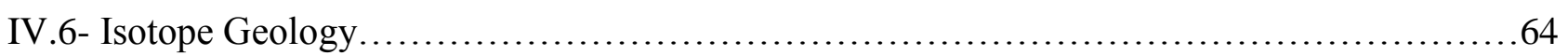

IV.6.1- Zircon Characteristics.....................................................64

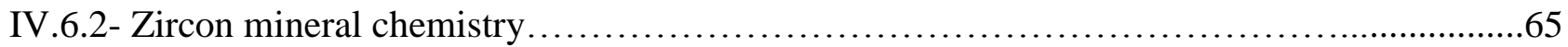

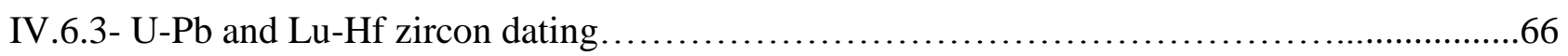

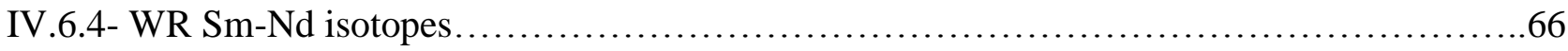

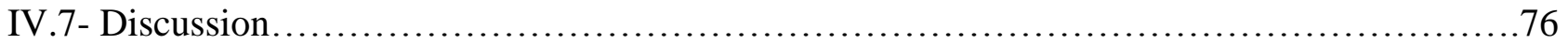

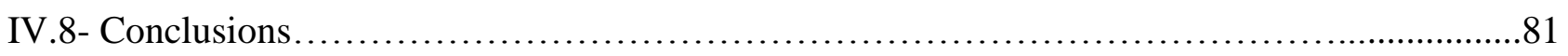

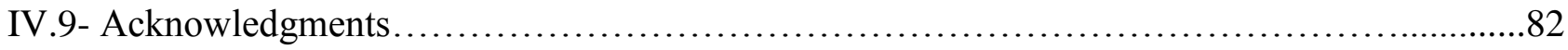

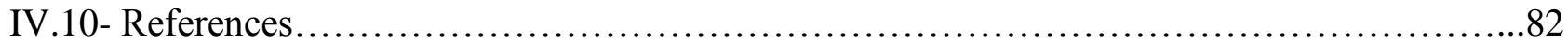


V- Capítulo - II Artigo.

V -“The Age of Gold Mineralization Hosted by Orosirian Granite at Trairão Deposit - Alta Floresta Gold Province (Amazonian Craton): Constraints from U-Th-Pb SHRIMP Dating of Hydrothermal Monazite" ........................................................91

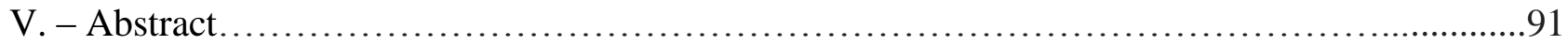

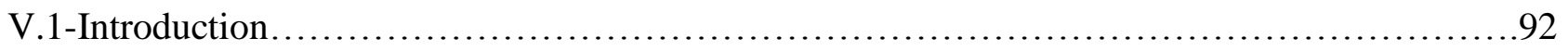

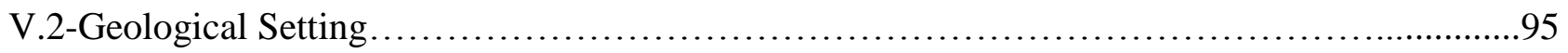

V.2.1- Caracterization of the Analysed Samples.........................................96

V.2.2- Hydrothermal Alteration and Breccia Type.........................................97

V.3-Analitical Procedures.......................................................... 99

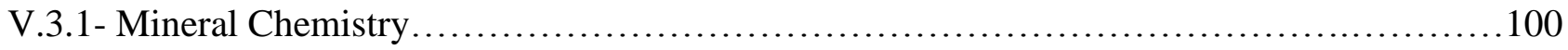

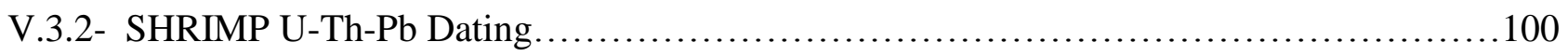

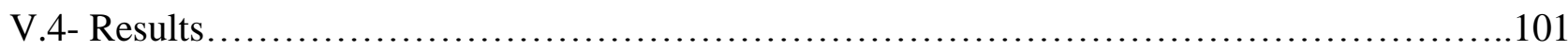

V.4.1-U-Pb-Th Results........................................................... 107

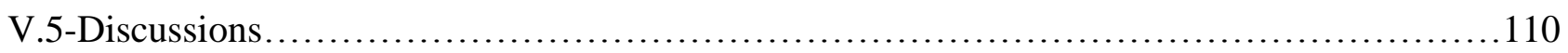

V.6-Conclusions.................................................................. 112

V.7 - Acknowledgments........................................................ 113

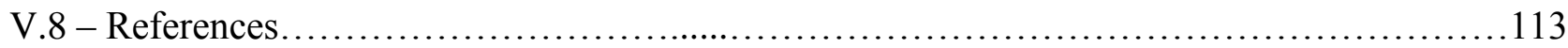

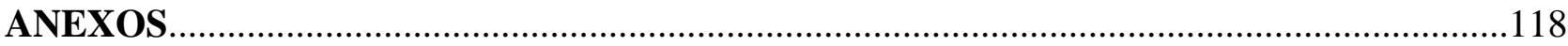




\section{LISTA DE FIGURAS}

Figura 01- Mapa de localização e vias de acesso, com detalhe da área de estudo. .08

Figura 02 - Mapa Geotectônico do Cráton Amazônico. (A) Modelo geotectônico do Cráton Amazônico de Santos et al. (2004); (B) Subdivisão da Província Tapajós-Parima em diferentes domínios metalogenético, Santos et al. (2008).

Figura 03 - Mapa Geológico das Províncias Auríferas de Alta Floresta e Tapajós, limitadas por bacias sedimentares ao norte e sul (extraído de Paes de Barros, 2007). Na legenda encontam-se os principais domínios tectônicos e seus repectivos domínios geológicos.

Figura 04 - Mapa Geológico da região da Província Aurífera de Alta Floresta. Adaptado de Lacerda Filho et. al. (2004), Souza et. al. (2005), Vasques \& Rosa-Costa (2008), Silva \& Abram (2008) e Alves et. al. (2010). Destaca-se que os locais de ocorrência de depósitos de ouro estão alinhados N80WS80E, segundo lineamento Peru-Trairão (LPT).

Figura 05 - Mapa geológico regional da parte leste da PAAF, com localização da área de pesquisa, modificado de Miguel Jr. 2011......

Figura 06 - A - Forma de ocorrência dos afloramentos, formando colina suave; B- Dique de aplito cortando fácies de granulação média do biotita sienogranito; C- Granito rosado, granulação média; DDetalhe do biotita monzogranito isotrópico.

Figura 07 - A- Afloramento de biotita sienogranito de granulação fina a média; B- Enclaves de composição intermediária a ácida; C- Dique de diabásio; D- Sulfetos associados a veios de quartzo, próximo à zona mineralizada.

Figura 08 - Esboço geológico regional, modificado de Miguel Jr (2011), com a área de pesquisa. Dados $\mathrm{U}-\mathrm{Pb}$ obtidos no presente trabalho.

Figura 09- (A) fenocristal de feldspato potássico com intercrescimento pertítico (nicóis cruzados); (B) fenocristal de feldspato potássico com textura micropertítica em matriz fina composta por cristais de quartzo recristalizados (nicóis paralelos); (C) agregado de biotita, clorita secundária e minerias opacos (nicóiss paralelos); (D) fenocristal de microclínio com vestígio de geminação xadre em matriz composta por cristais de quartzo com contatos irregulares (nicóis paralelos).

Figura 10- Rocha com textura ígnea granular com cristais de plagioclásio (oligoclásio), feldspato potássico com aspecto turvo, quartzo e biotita intensamente alterada para clorita; (B,C) Cristais de plagioclásio com geminação polissintética e saussuritizados, biotita intersticial e epidoto secundário; (D) cristais de plagioclásio saussuritizados com múltiplas geminações e com inclusões, textura poiquilítica. (todas as fotomicrografias em nicóis cruzados).

Figura 11 - (A,B); cristal sub-édrico de hornblenda com bordas corroídas (nicóis paralelos). .34 
Figura 12- A - fotomicrografia de dique de diábasio com textura sub-ofítica, cristais tabulares de plagioclásio geminados (nicóis paralelos); B- clinopiroxênico (augita) com macla simples, plagioclásio e minerais opacos (nicóis cruzados).

Figura 13 - (A - B) Os cristais cúbicos e sub-angulares de pirita, cor amarelo claro, intensamente alterada; (C) pequenos cristais de pirita cúbica disseminadas na rocha; (D) cristal sub-édricos de ilmenita, cor rosa pálido a amarelado.

Figura 14 - (A-B) Alteração secundária pervasiva em minerais de plagioclásio, caracterizada por clorita + sericita + carbonato + epidoto \pm pirita. (nicóis cruzados).

IV Figure 01 - Amazonian Craton geotectonic maps. (A) Geotectonic model by Santos et al. (2003); (B) Different metallogenic sub- domains of the Tapajós-Parima Province, Santos et al. (2008)....

IV Figure 02 - Regional geological map of the eastern part of the AFGP, with location of the study area, modified Miguel Jr. et al. 2011

IV Figure 03 - (A) Exposures of granites blocks in the field; (B) Fine-grained syenogranite; (C) biotite syenogranite; (D) Aplite Dyke cutting the biotite monzogranite; (E) Hyddrothermal Breccia in the gold deposit; (F) Gold-digging deactivated. ...

IV Figure 04 -Geochemical classification diagram and magmatic series. (A) $\mathrm{SiO}_{2}$ versus $\mathrm{K}_{2} \mathrm{O}$ (Peccerillo \& Taylor 1976, 1989); (B) ANK versus ACNK diagram of Maniar \& Piccoli (1989) with peraluminous index of Shand (1943). Nhandu I. S. (blue) and Matupá I. S. (red).

IV Figure 05 - (A-B) Multielement diagram for analyzed granite samples normalized to chondrite (Thompson, 1982). (C-D) REE patterns normalized to chondrite after Nakamura *1974). Nhandu Intrusive Suite (blue) and Matupá Intrusive Suite (red).

IV Figure 06- (A) Tectonic setting discrimination diagram with $\mathrm{Rb}$ versus $\mathrm{Y}+\mathrm{Nb}$ (Pearce et al, 1984, Pearce 1996 ); (B) Tectonic discrimination diagram, Harris et al. (1986). Nhandu Intrusive Suite (blue) and Matupá Intrusive Suite (red).

IV Figure 07- BSE image of first population of zircon crystals, TRBY- 32 and TRBY-08 samples, showing their morphology. Red points indicate the locations analyzed by WDS... . .64

IV Figura 08 - BSE image of seconf population of zircon crystals, TRBY- 20 and TRBY- 25 samples, showing their morphology. Red points indicate the locations analyzed by WDS. . .65

IV Figure 09 -Concordia diagram U/Pb (SHRIMP) for Nhandu Intrusive Suite, (A) sample TRBY -32, showing a crystallization age of $1946 \pm 21 \mathrm{Ma}$; (B) Concordia diagram U/Pb (SHRIMP) from sample TRBY -08, revealing a concordante age by the superior intercept of $1969 \pm 7.3 \mathrm{Ma}$; (C) Concordia diagram U / Pb (SHRIMP) sample TRBY -32F, 1954.5 $\pm 4.5 \mathrm{Ma}$

IV Figure 10 -Concordia diagram U/Pb (SHRIMP) for Matupá Intrusive Suite, (A) sample TRBY-25, crystallization age of $1837 \pm 13 \mathrm{Ma}$ and heritage with age $1960 \pm 18 \mathrm{Ma}$; (B) Concordia diagram U/Pb (SHRIMP) of TRBY -20 sample showing crystallization age of $1851 \pm 12 \mathrm{Ma}$; (C) Concordia diagram 
$\mathrm{U} / \mathrm{Pb}$ (SHRIMP) sample TRBY-27 crystallization age of $1878.4 \pm 7.5 \mathrm{Ma}$ and heritage with age 1923 $\pm 17 \mathrm{Ma}$.

IV Figure 11- A) Variation of Th versus U ratios for zircons from Trairão region; (B) Th/U versus $\mathrm{Zr} / \mathrm{Hf}$ ratios; (C) U/Yb versus $\mathrm{Y}$, (D) U/Yb versus Hf diagrams with the fields of Grimes et al. (2007) to discriminate between continental and oceanic crust zircon. Heavy lines Indicate the lower limit of zircons from continental crust.

IV Figure 12- Diagram of $\varepsilon H f$ values ( $\mathrm{t}$ ) and Hf isotopic evolution, where blue $=$ Transmazonian crust evolution, green $=$ Archean crust evolution . 78

IV Figure 13- ENd diagram for granitic rocks of the Matupá Intrusive Suites (red) and Nhandu (blue) in the Trairão region, Mundo Novo, MT.

V Figure 01 - Location of the geochronological and tectonic provinces of the Amazonian Craton according the: (A) Tassinari \& Macambira (1999) and (B) Santos et al. (2008) models

V Figure 02 - Geological map of part of Alta Floresta Gold Province. The insert is the study area where the Trairão Au-deposits, modified from Miguel Jr. (2011)

V Figure 03 - (A) Panoramic view of the Trairão deposit region; (B) Mineralized veins crosscut the host rock, hydrothermal breccia in biotite syenogranite; (C) comb structure quartz-vein, that crosscut the granite Trairão; (D) detail of mineralized sample. .98

V Figure 04 - (A-B) secondary pervasive alteration (sericitic) in plagioclase minerals (sample TR-08 $\mathrm{S})$, characterized by chlorite + sericite + carbonate + epidote \pm pyrite. (crossed nicols). .99

V Figure 05 - Representative backscattered electron images of thin section, sample TR-20T and TR$18 \mathrm{~S}$, showing monazite in apparent textural equilibrium with other minerals as barite, chalcopyrite and ilmenite, in the matrix of ore-bearing zone of Trairão and Chumbo Grosso deposit..... 102

V Figure 06 - Scanning electron microscope backscattered electron (BSE) photomicrographs imagens of sample TR-20T and TR-18, of cross-sections of representative monazites. SHRIMP analytical areas are indicated by circles and are approximately $10 \mu \mathrm{m}$ in diameter

V Figure 07 - Representative rare earth element (REE) distribution patterns for monazites from

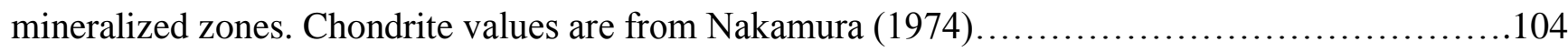

V Figure 08 - Bivariate diagrams for trace elements for monazites from mineralized zones

V Figure 09 - Concordia diagram of SHRIMP II data for monazite from the Au-mineralized veins at the Trairão deposit. Shown in the inset, is $1812 \pm 18 \mathrm{Ma}(\mathrm{MSWD}=2.5) .$. 108

V Figure 10 - Concordia diagram of SHRIMP II data for magmatic monazite from the ore rock. Shown in the inset, is $1825 \pm 27 \mathrm{Ma}(\mathrm{MSWD}=0.2)$ .108

V Figure 11 - Concordia diagram of SHRIMP II data for magmatic zircon sample TR-20. Shown in the inset, is $1851 \pm 12 \mathrm{Ma}$ interpreted as the crystallization age of the granitic massive (MSWD =2.4)...109 


\section{LISTA DE TABELAS}

IV Table 01 - Geochemical analyses of rocks from Nhandu Intrusive Suite and Matupá Intrusive Suite at the Novo Mundo region (MT). Oxides in \% and trace and rare earth elements in ppm

IV Table 02 - Chemical mineral analyses of zircon grains from Nhandu Intrusive Suite at the Novo Mundo region (MT).

IV Table 03 - Chemical mineral analyses of zircon grains from Matupá Intrusive Suite at the Novo

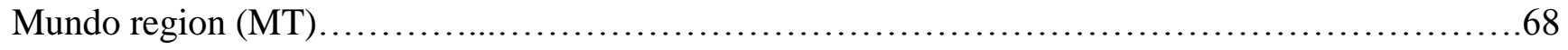

IV Table 04- SHRIMP U-Pb zircon data of Nhandu granitic rocks............................71

IV Table 05- SHRIMP U-Pb zircon data of Matupá granitic rocks.............................72

IV Table 06 - Lu-Hf isotopic data of zircons from Nhandu granitic rocks, Novo Mundo, Mato Grosso, Brazil.

IV Table 07 - Lu-Hf isotopic data of zircons from Matupá granitic rocks, Novo Mundo, Mato Grosso, Brazil.

IV Table 08 - WR Sm-Nd isotopes for Nhandu and Matupá Intrusive Suite samples, Novo Mundo, Mato Grosso, Brazil..... .75

$\mathrm{V}$ Table 01 - Microprobe analyses and oxide formula from the grains of monazite, samples TR - 20T and TR $-18 \mathrm{~S}$, value are present in percentage. 105

V Table 02 - SHRIMP II, U-Th-Pb isotopic data for monazites from, samples TR - 20T (Trairão Deposit) and TR - 18S (Chumbo Grosso Deposit).

V Table 03 - Description of samples from Trairão and Chumbo Grosso Deposits. Minerals selected for SHRIMP II analyses. 


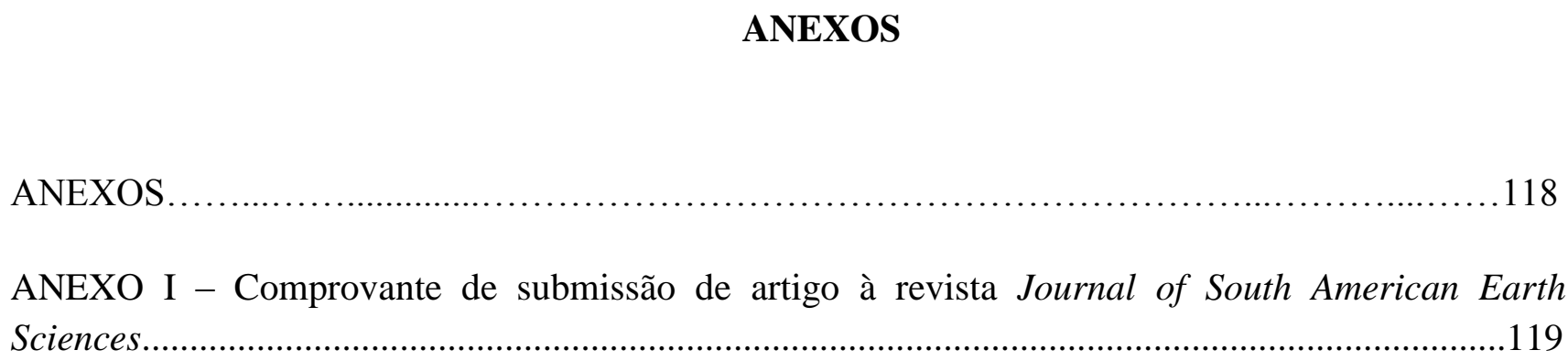

ANEXO II - Comprovante de submissão de artigo à revista Chemical Mineralogy..... 120

ANEXO III - Mapa de Localização das amostras datadas.

ANEXO IV - Diagrama de densidade de probabilidade para $\varepsilon$ Hf (T) em zircão do Suíte Intrusiva Nhandu e da Suíte Intrusiva Matupá.

ANEXO V - Diagrama de densidade de probabilidade para $\mathrm{T}_{\mathrm{DM}}(\mathrm{Ma})$ em zircão da Suíte Intrusiva Nhandu e da Suíte Intrusiva Matupá. 
LISTA DE ABREVIATURAS

\begin{tabular}{|c|c|c|c|}
\hline PAC & Província Amazônia Central & PAAF & Província Aurífera de Alta Floresta \\
\hline PMI & Província Maroni-Itacaiúnas & $\mathrm{CA}$ & Cráton Amazonas \\
\hline PVT & Província Ventuari-Tapajós & BSE & Elétrons Retroespalhados \\
\hline PRNJ & Provínca Rio Negro-Juruena & PPM & Porcentagem por milhão \\
\hline PRDI & Rondonian-San Ignácio & MT & Mato Grosso \\
\hline PS & Província Sunsás & ETR & Elementos terras raras \\
\hline PCI & Província Carajás-Imatacá & $\mathrm{Ga}$ & Giga anos \\
\hline PT & Província Tranzamazônica & $\mathrm{Ma}$ & Milhões de anos \\
\hline PTP & Província Tapajós-Parima & GPS & Global Positioning System \\
\hline PAC & Província Amazônia Central & UWA & University of Western Australia \\
\hline PRN & Província Rio Negro & $\mathrm{Km}$ & Kilômetros \\
\hline PRJ & Província Rondônia-Juruena & $\mathrm{mm}$ & Milímetros \\
\hline PKM & Província K’Mudku & $\mathrm{cm}$ & Centímetros \\
\hline DJ & Domínio Jamari & $\mathrm{N}$ & Norte \\
\hline DAF & Domínio Alta Floresta & $\mathrm{NE}$ & Nordeste \\
\hline DAJ & Domínio Alto Jauru & NW & Noroeste \\
\hline $\mathrm{Ta}$ & Tântalo & $S$ & Sul \\
\hline Hf & Háfnio & SE & Sudeste \\
\hline $\mathrm{La}$ & Lantânio & SW & Sudoeste \\
\hline $\mathrm{Ce}$ & Cério & $\mathrm{U}-\mathrm{Pb}$ & Urânio-chumbo \\
\hline $\mathrm{Nd}$ & Neodímio & $\mathrm{Pb}-\mathrm{Pb}$ & Chumbo-chumbo \\
\hline $\mathrm{Sm}$ & Samário & $\mathrm{SiO}_{2}$ & Dióxido de sílica \\
\hline $\mathrm{Eu}$ & Európio & $\mathrm{Al}_{2} \mathrm{O}_{3}$ & Óxido de alumínio \\
\hline $\mathrm{Gd}$ & Gadolínio & $\mathrm{Fe}_{2} \mathrm{O}_{3}$ & Óxido de ferro \\
\hline Dy & Disprósio & $\mathrm{CaO}$ & Óxido de cálcio \\
\hline Ho & Hólmio & $\mathrm{TiO}_{2}$ & Dióxido de titânio \\
\hline $\mathrm{Er}$ & Érbio & $\mathrm{P}_{2} \mathrm{O}_{5}$ & Óxido de fósforo \\
\hline $\mathrm{Yb}$ & Itérbio & WR & Whole rock \\
\hline
\end{tabular}




\begin{tabular}{|l|l|l|l|} 
Lu & Lutécio & $\mathrm{MgO}$ & Óxido de magnésio \\
$\mathrm{SIM}$ & Energy Dispersive System & $\mathrm{Pb}$ & Chumbo \\
$\mathrm{SIN}$ & Suíte Intrusiva Matupá & $\mathrm{Cu}$ & Cobre \\
$\mathrm{BC}$ & Bacia do Caiabis & LA-ICP-MS & Laser ablation inductively coupled \\
DJR & Domínio Juruena & $\mathrm{Nx}$ & Sensitive high resolution ion micro-probe \\
DT & Domínio Tapajós & $\mathrm{Np}$ & Nicóis cruzados paralelos \\
CL & Cátodo Luminescência & AFGP & Alta Floresta Gold Province \\
LPT & Lineamento Peru-Tairão & CMCA & $\begin{array}{l}\text { Center of de Microscopy, Characterization } \\
\text { and Microanalysis }\end{array}$ \\
\hline
\end{tabular}




\section{RESUMO}

Os garimpos Trairão e Chumbo Grosso, situados na Província Aurífera de Alta Floresta, consistem em depósitos de ouro que ocorrem em veios de quartzo e brechas vulcânicas associados a intrusões félsicas. Estudos petrográficos, geoquímicos e isotópicos foram realizados nas rochas encaixantes e mineralizadas para estabelecer a idade dos processos magmáticos e hidrotermais do depósito aurífero. As rochas magmáticas contêm características petrográficas e afinidade geoquímica da série cálcico-alcalina com alto potássio. Idades U-Pb em zircão pelo método SHRIMP permitem estabelecer ciclos magmáticos de $2000 \mathrm{Ma}, 1,969$ - 1,923Ma e 1,878 - 1,837Ma na região do garimpo, correlacionado ao Complexo Cuiú-Cuiú e às Suítes Intrusivas Nhandu e Matupá, respectivamente. A assinatura isotópica obtida por Sm-Nd e Lu-Hf, com valores de épsilon $\mathrm{Nd}(\mathrm{t}=$ tempo de cristalização) variando de $+0,05$ a $-6,42$ sugerem fontes mantélicas para rocha total com participação de material crustal em diferentes proportions, enquanto valores de épsilon Hf de $-3,9$ a -9,5 em zircão apontam que este tenha um maior tempo de residência para estes minerais (ou maior participação de componente crustal em sua composição). Os resultados de estudos químicos nas monazitas ocorrentes nas zonas de mineralizações auríferas associadas a granitos mostram teores variáveis de $\mathrm{Th}$ e $\mathrm{U}$ e com maior enriquecimento de $\mathrm{Ce}$ com respeito à La, assim como morfologia compatível com monazitas hidrotermais. Análises isotópicas de $\mathrm{U}-\mathrm{Th}-\mathrm{Pb}$ em monazitas hidrotermais permitem estabelecer valores de 1812 \pm 18 Ma e $1825 \pm 13$ Ma para os depósitos de Trairão e Chumbo Grosso, que sugerem que parte das mineralizações estudadas estão encaixadas em corpos graníticos mais antigos e resultam da intrusão de magmas mais jovem gerados em torno de 1,81 - 1,82 $\mathrm{Ga}$. Consequentemente, as idades obtidas a partir de dados U-Th-Pb em monazita hidrotermal provenientes do minério brechado podem ser consideradas uma boa referência para a formação de minério de $\mathrm{Au}$ nos depósitos do Trairão, associada a processos pós-magmáticos e contemporâneos com a cristalização da sericita nas brechas mineralizadas.Com os dados obtidos até o momento, propõe-se que as rochas magmáticas estudadas neste trabalho tenham sido formadas em um ambiente de arco magmático continental e que as mineralizações auríferas da PAAF na região do Garimpo Tairão foram geradas por pelo menos duas fases hidrotermais distintas. A mais antiga formou-se durante a intrusão do granito Matupá (1860 Ma) e mais jovem no período entre 1812 e 1825 Ma. O processo deve ter sido gerado durante a ascenção de pluma mantélica que forneceu calor para fusões crustais e ascensão de granitos 
epizonais mineralizados associados à fase magmática dos granitos da Suíte Intrusiva Juruena, mais antigos que o Grupo Colíder e Teles Pires.

PALAVRAS-CHAVE: zircão, monazita, datação $U-P b$, depósito aurífero hidrotermal, Província Alta Floresta, Cráton Amazônico.

\section{ABSTRACT}

The Trairão and Chumbo Grosso deposits, located in Alta Floresta Gold Province, consists of a gold mineralization in quartz veins and volcanic breccias associated with felsic intrusions. Petrographic, geochemical and isotopic studies in the mineralized and wall rocks were carried out to establish the age of the magmatic and hydrothermal processes of the gold deposit. The host magmatic rocks of the Au-mineralization are representd by the Matupá Intrusive Suite, which it intrudes at the Trairão region the Nhandu Intrusive Suite. These suites contain petrographic features and geochemical affinity of the calc-alkaline series with high potassium. SHRIMP U-Pb zircon dating on these granitcs rocks allow to establish magmatic cycles of $\sim 2000 \mathrm{Ma}, 1.969-1.923 \mathrm{Ma}$ and $1.878-1.837 \mathrm{Ma}$ at the Trairão region correlated to the Cuiú-Cuiú complex and Matupá and Nhandu Intrusive Suite. The isotopic signature obtained by Sm-Nd and Lu$\mathrm{Hf}$, with epsilon $\mathrm{Nd}(\mathrm{t})$ values $(\mathrm{t}=$ crystallization time) ranging from +0.05 to -6.42 suggest mantle sources for whole rock with some participation of crustal material, while epsilon $\mathrm{Hf}(\mathrm{t})$ values on zircon from -3.9 to -9.5 point to a longer residence time (or greater participation of crustal component in its composition). The results of chemical studies in the monazite occurring in auriferous mineralization deposits associated with granites show variable Th and $\mathrm{U}$ values and higher concentration of $\mathrm{Ce}$ with respect to La, as well as morphology compatible with hydrothermal monazite. Isotopic analysis of U-Th-Pb hydrothermal monazite of the Trairão and Chumbo Grosso deposits yielded ages of $1812 \pm 18 \mathrm{Ma}$ e $1825 \pm 13 \mathrm{Ma}$ respectively, suggesting that part of the mineralization studied hosted in the older granitic bodies and are probable connected with younger magmas intrusion generated around 1.81-1.82 Ga. . Consequently, the ages obtained from $\mathrm{U}$ data $-\mathrm{Th}-\mathrm{Pb}$ hydrothermal monazite from the breccia ore can be considered a good reference for Au ore formation in Trairão deposits, associated with post-magmatic processes and contemporary with crystallization sericite in the breccia. With the data obtained so far, it is proposed that the magmatic rocks studied in this work have been formed in a continental magmatic arc environment and the auriferous mineralizations in Trairão mine were generated by at least two distinct hydrothermal 
stages. One associated with intrusion of Matupá Granite (1860 Ma) and another (1812$1825 \mathrm{Ma})$ associated with mineralized epizone granites of Intrusive Suite Juruena, older than the Colíder Group and Teles Pires.

KEYWORDS: zircon, monazite, U-Pb dating, hydrothermal Au-deposit, Alta Floresta Province, Amazonian Craton.

\section{ESTRUTURA E ORGANIZAÇÃO DA TESE}

O presente trabalho consiste de Tese do curso de Doutorado junto ao Programa de Pós-graduação em Geologia do Instituto de Geociências da Universidade de Brasília. A apresentação da tese segue o modelo de integração de artigos científicos, subdivididos em cinco capítulos. O Capítulo I é introdutório, no qual estão informações sobre a localização da área de estudo, os objetivos e as justificativas para a escolha do objeto de estudo e a descrição dos materiais e métodos empregados no desenvolvimento da tese; O Capítulo II apresenta uma revisão da Geologia Regional e Contexto Geológico do Cráton Amazônico, no qual são definidas as suas províncias com ênfase na Província Aurífera de Alta Floresta. O Capítulo III apresenta a Geologia Local da área do Garimpo Trairão e as descrições petrográficas, ao final desse capítulo estão às referências bibliográficas citadas anteriormente. O Capítulo IV é constituído pelo primeiro artigo submetido à revista científica Journal of South American Earth Sciences, intitulado "Isotope Geology of the Granitic Rocks from Trairão Deposit-Alta Floresta Gold Domain - Southern Amazonian Craton" e o Capítulo V apresenta o segundo artigo desenvolvido durante a pesquisa que será submetido à revista científica Chemical Geology, intitulado "The Age of Gold Mineralization Hosted by Orosirian Granite at Trairão Deposit - Alta Floresta Gold Province (Amazonian Craton): Constraints From U-Th-Pb SHRIMP Dating of Hydrothermal Monazite”. (Os anexos são colocados ao final da Tese). 


\section{CAPÍTULO - I INTRODUCÃ̃}




\section{I.1 - MOTIVAÇÃO AO TRABALHO E JUSTIFICATIVA}

A escolha da área de estudo, dentro da Província Aurífera Alta Floresta (PAAF), deve-se ao fato que a PAAF foi uma das mais importantes províncias metalogenéticas do Brasil, especialmente na produção de ouro. Estima-se que foram produzidos entre 1980 e 1998, cerca de 160 toneladas de ouro, colocando Mato Grosso entre um dos maiores produtores de ouro no Brasil. O entendimento geológico atual da área aponta para uma evolução por acresção de arcos e magmatismo pós-orogênico que iniciou sua evolução em 2,0 Ga e se estendeu até $1,75 \mathrm{Ga}$. As mineralizações que nas décadas de 80 e 90 foram exploradas em aluviões passaram a ser extraídas in situ, como de origem magmática, ligadas a vários eventos vulcano-plutônicos, principalmente félsicos e de afinidade cálcico-alcalino. Essas observações reforçam a similaridade da PAAF com ambientes de margem continental ativa tipoAndina. Entretanto, devido à série de dificuldades para desenvolver investigações científicas na região amazônica, o entendimento dos processos geológicos associados às mineralizações auríferas e suas hospedeiras da província tem ainda uma série de lacunas. Assim, a necessidade de obter mais dados para uma região tão importante em termos geológicos e metalogenéticos motivou a autora deste trabalho a escolher a área extensivamente minerada denominada de região do Garimpo Trairão. Estudos em detalhe sobre o ponto de vista de petrografia e geoquímica mineral e das rochas graníticas da área, com auxílio de ferramentas como geoquímica isotópica de Lu-Hf em zircão e Sm - Nd em rocha total são aplicados para obter melhor compreensão da afinidade geoquímica, da fonte dos magmas e do tempo de residência crustal. Associados a estes, o estudo geocronológico de $\mathrm{U}-\mathrm{Pb}-\mathrm{Th}$ em zircão e monazita, magmáticas e hidrotermais é utilizado no presente trabalho para determinar a idade das rochas graníticas e dos depósitos auríferos. (A integração de todos os dados é, portanto, realizada para obter um melhor entendimento da idade da mineralização e de geração das rochas encaixantes). 


\section{I.2 - APRESENTAÇÃO}

A Província Aurífera de Alta Floresta - PAAF (Dardene \& Schobbenhaus 2001), Província Aurífera Juruena - Teles Pires (Silva \& Abram 2008), Domínio Alta Floresta (Santos et al. 2001), ou Província Mineral de Alta Floresta (Souza et al., 2005), está inserida na porção sul do Cráton Amazônico, configurando uma área alongada na direção NWSE limitada a norte pelo gráben do Cachimbo, que a separa da Província Aurífera do Tapajós, e a sul pelo gráben dos Caiabis.

É considerada como parte de diferentes províncias geocronológicas, no modelo de Santos et al. (2000) está inserida entre as Províncias Tapajós - Parima (2,03-1,88 Ga) e Rondônia - Juruena (1,82-1,54 Ga), ou de acordo com Tassinari \& Macambira (1999), entre as Províncias Ventuari - Tapajós (1,95-1,8 Ga) e Rio Negro - Juruena (1,8-1,55 Ga). Independente do modelo adotado, as províncias são compostas por unidades plutono-vulcânicas que foram geradas em ambiente de arcos magmáticos, que se desenvolveram e se agregaram progressivamente ao protocráton formado pela Província Amazônia Central no Paleoproterozóico (Tassinari \& Macambira 1999; Souza et al. 2005; Santos et al. 2006; Silva \& Abram 2008).

A Província Aurífera de Alta Floresta apresenta um potencial aurífero demonstrado por sua produção de ouro, na ordem de 160 t, extraído entre os períodos de 1980 e 1999, sendo considerada uma das principais regiões produtoras do metal no país, principalmente por atividade garimpeira em depósitos aluvionares e posteriormente por depósitos primários (Paes de Barros, 2007). A história magmática dos granitóides e sua mineralização associada, tem sido tema frequente de diversas investigações. Hipóteses de depósitos de ouro relacionados a granitos têm sido propostas como de origem do tipo Lode orogenético mesotermais (Paes de Barros, 1994; Santos et al., 2001; Teixeira, 2015); depósitos epizonais - intrusion-related gold systems (Santos et al., 2001; Paes de Barros, 2007; Abreu, 2004; Bizotto, 2004); ouro pórfiro (Moura et al.1998; Moura et al., 2006; Assis, 2011; Trevisan, 2012; Trevisan, 2015) e mineralizações epitermais (Assis, 2011; Trevisan, 2015). A compreensão da evolução geotectônica do cráton amazônico é de fundamental importância para o entendimento dos processos metalogenéticos, sendo fundamental a caracterização petrológica e isotópica das rochas magmáticas que hospedam tais mineralizações.

Nesse contexto, este trabalho enfatiza a caracterização petrográfica, geoquímica e geocronológica dos corpos graníticos associados à ocorrência de ouro na região do 
garimpo Trairão e Chumbo Grosso, município de Novo Mundo, oeste da Província Aurífera de Alta Floresta. Idades U-Pb em zircão e monazita (SHRIMP) e dados isotópicos de $\mathrm{Lu}-\mathrm{Hf}$ e $\mathrm{Sm}-\mathrm{Nd}$ inéditos são apresentados para amostras da região estudada.

\section{I.3 - OBJETIVOS}

O presente trabalho tem como objetivo estabelecer as idades dos depósitos auríferos Trairão e Chumbo Grosso, que tem como rochas hospedeiras as rochas sienograníticas da Suite Intrusiva Matupá, para definir melhor o(s) período(s) de mineralização na porção Leste da Província Aurífera de Alta Floresta (PAAF), MT, e sua relação com as rochas encaixantes graníticas. Estudos de petrografia, geoquímica e geologia isotópica nas monazitas hifrotermais associadas às mineralizações auríferas e nas rochas graníticas encaixantes, representadas pela suites intrusivas Nhandu e Matupá, são apresentados para caracterizar o seu ambiente tectônico e os processos de acresção crustais na PAAF.

\section{I.4 - LOCALIZAÇÃO E VIAS DE ACESSO}

A área de estudo (Figura 1) está localizada na porção Centro-Norte do estado de Mato Grosso, município de Novo Mundo, na região do curso do rio Trairão afluente do rio Teles Pires. O acesso é realizado partindo de Cuiabá, capital de Mato Grosso (Figura 1), pela BR - 163 (Cuiabá-Santarém) por aproximadamente $720 \mathrm{~km}$, passando pelos municípios de Nobres, Nova Mutum, Lucas do Rio Verde, Sorriso, Sinop, Peixoto de Azevedo, Guarantã do Norte até o Município de Novo Mundo-MT. O acesso ao garimpo do Trairão é realizado por estradas vicinais não pavimentadas, distando $32 \mathrm{Km}$ da área urbana do município de Novo Mundo. 


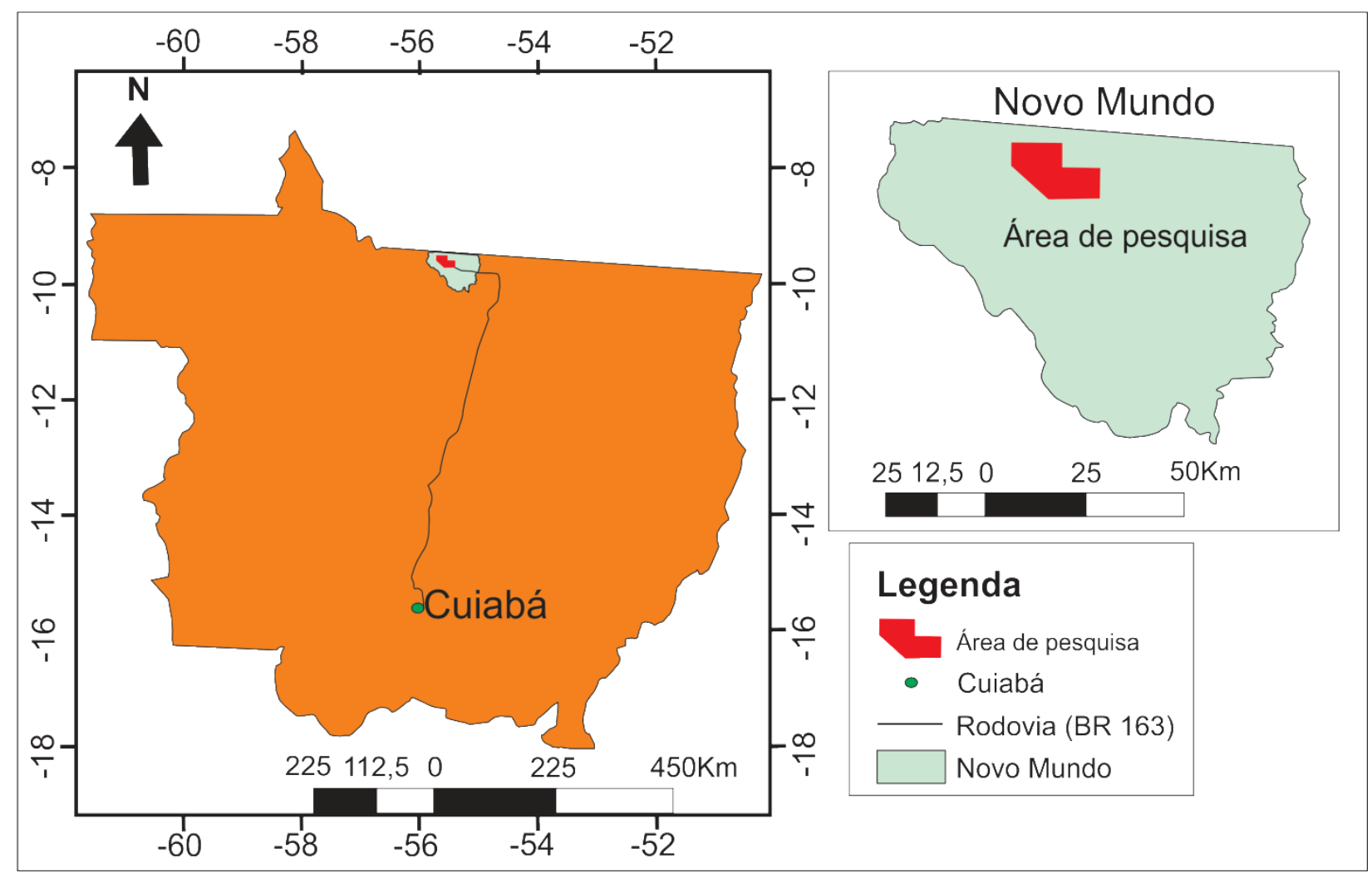

Figura 01- Mapa de localização e vias de acesso, com detalhe da área de estudo.

\section{I.5 - MÉTODOS E MATERIAIS}

Na primeira etapa deste trabalho foi feita uma revisão bibliográfica de teses e artigos científicos com a finalidade de conhecer e entender melhor os ambientes e sistemas evolutivos relacionados aos granitos dessa parte do Cráton Amazônico.

Com o auxílio de imagens de satélite foi realizado um reconhecimento geológico em escala 1:100.000, de uma área de aproximadamente $80 \mathrm{Km}^{2}$ na região do Munícipio de Novo Mundo -MT, com o objetivo de reconhecer as principais unidades litológicas e estruturais da área. A classificação e descrição petrográfica das fácies individualizadas, foi efetuada com o auxílio de lâminas delgadas e polidas e análises de geoquímica em rocha total das amostras mais representativas da região do Garimpo Trairão.

\section{I.5.1 - Petrografia}

A partir das amostras coletadas nas etapas de campo foram confeccionadas 30 lâminas e 20 seções polidas, produzidas no Laboratório de Laminação do Instituto de 
Geociências da UnB - IG/UnB e no Laboratório de Laminação da University of Western Australia (UWA).

O estudo petrográfico das lâminas e seções foi realizado utilizando microscópios petrográficos Zeiss Imager A2m e Olympus BX60. Este estudo proporcionou a observação dos minerais, textura, relações de contato entre os grãos, e alteração dos minerais. Ainda nesta etapa foram tiradas fotomicrografia das lâminas.

\section{I.5.2 - Geoquímica}

As amostras destinadas a análise geoquímica dos elementos maiores, menores, traços, Elementos Terras Raras (ETRs). Para realizar este tipo de analise as amostras foram pulverizadas em panela de ágata para evitar a contaminação por tungstênio, cobalto e cromo.

As análises químicas dos elementos maiores, menores, traços e terras raras (ETR) foram feitas no Acme Analytical Laboratories Ltd. (AcmeLabs), no Canadá. Os elementos maiores foram analisados por Inductively Coupled Plasma Atomic Emission Spectrometry (ICP-AES), após fusão com metaborato de lítio $\left(\mathrm{LiBO}_{2}\right)$. Os elementos menores e as terras raras foram analisados por Inductively Coupled Plasma Emission Mass Spectrometry (ICP-MS), após o mesmo tipo de fusão usada nos elementos maiores, exceto para os metais base e preciosos, cuja extração foi feita por digestão com água régia.

\section{I.5.3 - Microscopia Eletrônica - Química Mineral em Monazita}

Análises composicionais de monazitas de áreas com intensa alteração hidrotermal da região do Garimpo do Trairão, foram adquiridos em uma microssonda eletrônica (JEOL JXA8530F) equipado com 5 comprimentos de onda sintonizável à espectrômetros dispersivos. As condições operacionais foram, 40 graus de ângulo de decolagem, energia do feixe de $20 \mathrm{keV}$. A corrente do feixe de $130 \mathrm{nA}$, o diâmetro do feixe foi totalmente focalizado.

Elementos foram adquiridos utilizando análise de cristais LiF para Dy, Pr, Er, Nd, Gd, Sm, Yb, Eu, Peth para Th, Pb, PETJ para U, La, Y, Ce, Ca, P, Peth para Th, Pb, e TAP para Si. 
Os padrões foram Crocoite para $\mathrm{Pb}$, Monazita para $\mathrm{Th}$, Eu, Wollastonita para $\mathrm{Ca}, \mathrm{Si}$, Uraninita para $\mathrm{U}, \mathrm{YPO}_{4}$ para $\mathrm{P}, \mathrm{Y}, \mathrm{La} \mathrm{PO}_{4}$ para $\mathrm{La}, \mathrm{CePO}_{4}$ para $\mathrm{Ce}, \mathrm{PrPO} 4$ para $\mathrm{Pr}, \mathrm{NdPO}_{4}$ de Nd, $\mathrm{SmPO}_{4}$ para $\mathrm{Sm}, \mathrm{GdPO}_{4}$ para Gd, DyPO 4 para Dy, $\mathrm{ErPO}_{4}$ para Er, e $\mathrm{YbPO}_{4}$ para $\mathrm{Yb}$.

O tempo de contagem foi de 20 segundos para $\mathrm{Pr}, \mathrm{Nd}, \mathrm{Gd}, \mathrm{Sm}, \mathrm{Yb}, \mathrm{La}, \mathrm{Ce}, \mathrm{Ca}$, P; 40 segundos para Dy, Y, Er, 100 segundos para Si, e 200 segundos para $\mathrm{Pb}$, Th, U.

Os dados de intensidade de background (MAN) foram calibrados e absorção contínua corrigido para U, Si, Pb, Th, Dy, Pr, Er, Nd, Gd, Sm, Yb, La, Y, Ce, Ca, P, Eu. Intensidades desconhecidos e padrões foram corrigidos para deadtime.

Correções das interferências foram aplicados a $U$ de interferência causada por Th, Pr, Sm, Ce, e Si de interferência causada por Nd, Er, e Pb de interferência causada por Y, Th, e Th de interferência causada por Gd, Y, e Dy de interferência causada por Th, e Pr de interferência causada por La, e Er de interferência causada por Nd, e $\mathrm{Nd}$ de interferência causada por $\mathrm{Pb}, \mathrm{Ce}$, Th, e Gd de interferência causada por $\mathrm{Nd}, \mathrm{Ce}, \mathrm{La}, \mathrm{e}$ Sm de interferência por Ce, e Yb de interferência causada por Dy, e La de interferência causada por Nd.

Os limites de detecção variaram de 0,002 por cento em peso para Si, 0,016 por cento em peso para Dy e 0,023 por cento em peso para Sm. A sensibilidade analítica (no nível de confiança de $99 \%$ ) foi de 0,270 por cento para o $\mathrm{P}, 1,043$ por cento para Sm, 1.621 por cento relativo para Dy e 0,4146 para o $\mathrm{Yb}$.

\section{I.5.4 - Microssonda Eletrônica - Química Mineral em Zircão}

Os cristais de zircão estudados provêm de 6 amostras da região do Garimpo do Trairão. Essas populações foram pré-selecionadas com lupa binocular, tendo sido escolhidos de 30 a 40 grãos com características morfológicas semelhantes por amostra para serem datadas pelo método U-Pb (SHRIMP/USP e SHRIMP II/ Curtin University of Technology) e posteriormente os mesmos grãos foram estudados por microssonda eletrônica.

Os grãos foram montados em lâmina circular de vidro sobre uma camada de cola epóxi. Após um período de 12 horas a cola estava seca e iniciou-se a etapa de 
lixamento, para retirar o excesso de cola do mount, Em seguida realizou-se o polimento com pasta de diamante, até a superfície de exposição do grão ficar bem lisa, seguindo a limpeza com acetona. O procedimento final foi à deposição de um filme de carbono sobre a lâmina.

As imagens de Elétrons Retro-Espalhados (BSE) e as análises pontuais qualitativas (Energy-Dispersive Spectrometry - EDS) e quantitativas (WavelenghtDispersive Spectrometry - WDS) foram adquiridas no Laboratório de Microssonda Eletrônica do Instituto de Geociências da Universidade de Brasília, com a microssonda JEOL JXA-8230 (Electron Probe Microanalyzer). As condições analíticas para elementos maiores foram de $15,0 \mathrm{kV}$ de voltagem e corrente de feixe de $10 \mathrm{nA}$ por $10 \mathrm{~s}$ ( no pico , $5 \mathrm{~s}$ para determinação de background), com condições de vácuo de menos de $1 \times 10^{-4} \mathrm{MPa}$ para elementos óxidos .Para o elemento Hf, as condições foram alteradas: $20.0 \mathrm{kV}, 20 \mathrm{nA}$ e $20 \mathrm{~s}$ até o pico . Para elementos traço, as condições foram $20,0 \mathrm{kV}$ de voltagem e $100 \mathrm{nA}$ de corrente de feixe por $100 \mathrm{~s}$ ( no pico , 50 s determinação de background) sob as mesmas condições de vácuo.

\section{I.5.5 - Método U-Pb (SHRIMP)}

O método $\mathrm{U} / \mathrm{Pb}$ em zircão é amplamente utilizado na reconstrução da escala do tempo de corpos graníticos pelo fato da difusão do chumbo ser desprezível em temperaturas magmáticas podendo reter informações relacionadas com a idade de cristalização em diferentes estágios de evolução magmática, fonte, ascensão e alojamento (Cherniak and Watson, 2001).

Os grãos de zircão separados para análises de isótopos foram obtidos através do processamento individual de amostras representativas do granito estudado, tal separação foi realizada em duas etapas, a primeira etapa no Centro de Pesquisas Geocronológicas da Universidade de São Paulo (CPGeo - IGc - USP), e a segunda etapa no laboratório de preparação de amostras da The University od Western Australia (UWA), seguindo procedimentos habituais de cada laboratório, como britagem, moagem em disco, peneiramento (peneira de 60 mesh) posteriormente as amostras foram lavadas para remover as frações de argila e silte.

$\mathrm{O}$ material restante, correspondente a areia fina e muito fina, foi seco e processado em dois líquidos pesados, LST (tungstato de sódio e lítio, densidade $=2,85$ ) 
e, TBE (tetra-bromo-etano, densidade $=3$ ). Os concentrados de minerais pesados foram separados em quatro fracções usando um separador magnético Frantz. Grãos de zircão foram escolhidos a partir da fracção menos magnética em 1 A e 5 graus de inclinação e, em seguida, montado em um disco de epóxi de 2,5 centímetros de diâmetro em conjunto com os padrões analíticos.

Os grãos foram posteriormente selecionados e colhidos manualmente. Estes foram depois montados em conjunto com o padrão BR-266 (559 Ma, com 903 ppm de U, Stern, 2001) em epóxi e polidos até expor as texturas internas dos cristais.

A seção (mount) foi polida e revestida com uma película de carbono para geração de imagens usando Microscópio Eletrônico de Varredura (MEV) uma JEOL 6400 no Center of de Microscopy, Characterization and Microanalysis (CMCA) da The University of Western Australia (UWA), posteriormente o revestimento de carbono foi removido e substituído por um revestimento de ouro para analises U-Pb/SHRIMP II.

Análises U-Pb SHRIMP II (Sensitive High Mass Resolution Ion MicroProbe) foram realizados na Curtin University of Technology em quatro sessões spot de 20 e 25 mm. Análises individuais são compostas de medição de nove massas repetida em cinco scans. As seguintes massas foram analisadas nos grãos de zircão: $\left(\mathrm{Zr}_{2} \mathrm{O}, \mathrm{Pb}^{204}\right.$, background, $\mathrm{Pb}^{206}, \mathrm{~Pb}^{207}, \mathrm{~Pb}^{208}, \mathrm{U}^{238}, \mathrm{ThO}^{248}, \mathrm{UO}^{254}$ ). Os padrões D23 e NBS-611 foram utilizados para identificar a posição do pico de $\mathrm{Pb}^{204}$, enquanto a calibração do conteúdo de U e a razão $\mathrm{Pb} / \mathrm{U}$ foram feitas usando o padrão de zircão BR-266 (559 Ma, com 903 ppm U, Stern, 2001). Os dados foram reduzidos utilizando o software SQUID 1.03 (Ludwig, 2001) e as idades calculadas usando Isoplot 3.0 (Ludwig. 2003).

\section{I.5.6 - Método $\mathrm{Lu}-\mathrm{Hf}$}

Todos os zircões foram montados seções circulares de epóxi com 2,5 cm de diâmetro e polidos até que os grãos fossem revelados. Imagens de cátodo luminescência (CL) dos zircões foram obtidas utilizando um microscópio eletrônico (JEOL JSM 5800) no laboratório de Geocronologia da USP, sendo a metodologia descrita em Sato et al. (2008).

Os zircões foram analisados primeiro pelo método U-Th-Pb com SHRIMP e, em seguida, com LA-MC-ICP-MS para Lu-Hf. Para Lu-Hf, cuja análise pontual foi realizada preferencialemnte no mesmo site ou ao mesmo domínio de idade concordante do zircão já analisados pelo método U-Pb / SHRIMP em zircão, totalizando 30 
determinações isotópicas de Hf realizadas em 4 amostras. Medições isotópicas de Lu-Hf foram subsequentemente realizadas pelo método LA-MC-ICPMS usando um Thermo Finnigan Neptune Multicoletor-ICPMS e um sistema de microssonda a laser Photo Machine Excimer. Para este trabalho foram analisadas as massas 171, 173, 174, 175, 176, 177, 178 e 179 simultaneamente em copos de Faraday com axial copo de Faraday para a massa 176 dados isotópicos. Foram adquiridos utilizando o modo estático e determinação de 50 ciclos de 1,054 s. Todos os valores foram corrigidos para valores em branco. Condições de funcionamento a laser é a potência de saída do laser 5-6,5 $\mathrm{J} / \mathrm{cm} 2$, taxa de repetição de $10 \mathrm{~Hz}$ e diâmetro do spot de $40 \mu \mathrm{m}$. Entrada de gás com o fluxo do líquido de arrefecimento (Ar) de 15 l/min, fluxo auxiliar (Ar) de 0,7 1/min, o fluxo de transporte de amostra de 0,75 1/min (Ar) no MC-ICP-MS e 0,22 (Ar) e 0,55 1/min (He) no laser.

Razões isotópicas e de fracionamento inter-elemental foram avaliadas com o uso do padrão zircão GJ-1 (GEMOC, Macquarie University) para cada conjunto de 12 amostras de zircão, das quais foram analisadas oito amostras desconhecidas e padrões internos Mudtank e 91500. Para corrigir a interferrência isobárica de Lu e Yb isótopos de massa 176, os isótopos $\mathrm{Yb}^{171}, \mathrm{Yb}^{173}$ e $\mathrm{Lu}^{175}$ foram monitorados simultaneamente durante cada análise. $\mathrm{O} \mathrm{Lu}^{176}$ e $\mathrm{Yb}^{176}$ foram calculados utilizando razões de 0,026549 para $\mathrm{Lu}^{176} / \mathrm{Lu}^{175}$ e de 1,123456 para $\mathrm{Yb}^{173} / \mathrm{Yb}^{171}$ de, como apresentado por Chu et al. (2002) e Thirwall \& Walder (1995), respectivamente. Os dados foram normalizados com a razão de $\mathrm{Hf}^{179} / \mathrm{Hf}^{177}$ igual a 0,7325 (Patchett et al., 1981), utilizando uma correção exponencial para o viés (bias) de massa. Durante o decurso da análise do padrão GJ-1 produziu a razão $\mathrm{Hf}^{176} / \mathrm{Hf}^{177}$ de $0,281997 \pm 19(\mathrm{n}=8,2 \mathrm{DP})$, que é quase idêntico ao valor recomendado pela Morel et al. (2008) de 0,282 mil \pm 5 . Para avaliar a precisão e a exatidão dos resultados da microssonda a laser é relatado aqui as análises de dois padrões de zircão como amostra desconhecida, 91500 e Mudtank, que reproduziram as razões $\mathrm{Hf}^{176} / \mathrm{Hf}^{177}$ de $0,282291 \pm 15(\mathrm{n}=8,2 \mathrm{DP})$ e $0,282479 \pm 19(\mathrm{n}=$ 8, 2DP), semelhante aos valores sugeridos pela Woodhead et al. (2005). Chemale et al. (2011) descrevem métodos e tratamento dos dados analíticos para análises de isótopos de Lu-Hf em zircão. 


\section{I.5.7 -Geoquímica Isotópica de Sm-Nd}

Amostras graníticas representativas da região do Garimpo Trairão foram selecionadas para análises isotópicas de $\mathrm{Sm}$ e $\mathrm{Nd}$, as quais foram realizadas no Laboratório de Geocronologia do IG/UnB. Oito amostras de rocha total foram pulverizadas, que, posteriormente, foram dissolvidas em frasco de PTFE (Fluoretileno politetarédrico) com adição de traçadores isotópicos combinados de $\mathrm{Sm}^{149}-\mathrm{Nd}^{150} \mathrm{em}$ uma mistura de $\mathrm{HF}_{-} \mathrm{HNO}_{3}$ e $\mathrm{HCl}$ 6N. Após a dissolução completa das amostras, $\mathrm{Sm}$ e Nd foram separados em colunas primárias e secundárias, de PTFE. A coluna primária com resina catiônica AG-50W-X8 (200-400 mesh) foi utilizada para separar elementos terras raras (ETR) com uso de ácido $\mathrm{HCl} 2,5 \mathrm{~N}$ e 6,0 N. As amostras com ETR foram secas e misturadas com $\mathrm{HNO}_{3} 7 \mathrm{~N}$. À amostra de REE adicionou-se 0,2 ml HCL 0,18 N e esta foi passada em coluna de PFTE com resina HDEHP n HDEHP LN-B50-A (100$200 \mu \mathrm{m}$ ), separando-se Nd e Sm com uso de HCL 0,18 N e 0,5 N (segundo Goia \& Pimentel, 2000). Sm e Nd foram também secos para forma sólida, sendo analisados em um espectrômetro de massa Finnigan MAT 262 com fonte termal de ionização, no modo estático com 120 razões de $\mathrm{Nd}$ normalizadas para $\mathrm{Nd}^{146} / \mathrm{Nd}^{144}=0,7219$ e 60 razões de Sm. Todas as análises foram ajustadas para variações instrumentais com medidas do padrão interno. A correção dos brancos de $\mathrm{Sm}$ e $\mathrm{Nd}$ foi insignificante para as composições isotópicas de $\mathrm{Nd}$ e razões $\mathrm{Sm} / \mathrm{Nd}$. Idade modelos de $\mathrm{Nd}\left(\mathrm{T}_{\mathrm{DM}}\right)$ foram calculadas seguindo o modelo de manto depletado de De Paolo (1981). 


\section{CAPÍTULO - II}

\section{GEOLOGIA REGIONAL E}

\section{CONTEXTO GEOTECTÔNICO}




\section{II.1 - CONTEXTO GEOLÓGICO GEOTECTÔNICO}

O Cráton Amazônico, compreende grande parte norte e noroeste da América do Sul, é limitado por faixas orogênicas neoproterozóicos, cuja a evolução geológica é um tanto complexa. Em termos gerais, o Craton Amazônico é dividido em província geocronológicas com base em dados de U-Pb em mineral, Ar-Ar em mineral e rocha e Sm-Nd em rocha total. De acordo com Tassinari \& Macambira (1999), está dividido em seis províncias geocronológicas: Amazônia Central - PAC (> 2,3 Ga); MaroniItacaiúnas - PMI (2,2-1,95 Ga); Ventuari-Tapajós - PVT (1,95-1,80 Ga), Rio NegroJuruena - PRNJ (1,8-1,55 Ga); Rondonian-San Ignácio - PRSI (1,55-1,3 Ga) e Sunsás PS (1,3-1,0 Ga). Trabalhos de Santos (2000) e Santos et al. (2008) propõem oito províncias geocronológicas, a saber: Carajás-Imatacá (3,0- 2,50 Ga), Tranzamazônica (2,25 a 2,0 Ga), Tapajós-Parima (TPP) (2,03 a 1,88 Ga), Amazônia Central (1,88 a 1,86 Ga), Rio Negro (1,86 a 1,52 Ga), Rondônia-Juruena (RJ) (1,75 a 1,47 Ga), K'Mudku $(1,2 \mathrm{Ga})$ e Sunsás $(1,33$ a $0,99 \mathrm{Ga})$. Estas províncias formam um trend NW-SE com núcleo central Arqueano, em torno do qual as províncias geocronológicas paleo e mesoproterozóicas, sucessivamente mais jovens, se agregaram por meio de acresções de arcos magmáticos e retrabalhamento crustal (Teixeira et al., 1989; Tassinari \& Macambira, 1999; Santos et al., 2000; 2006; 2008; Tassinari et al. 2000; Cordani \& Teixeira, 2009).

Na região do Cráton Amazônico há diversas províncias metalogenéticas que são definidas pela presença de uma ou mais mineralização em uma determinada região, que ou correspondem uma parte de uma Provícia Geocronológica, como por exemplo Província Mineral Carajás que ocorre na Província Geocronológica Amazônia Central de Tassinari \& Macambira (1999), ou estão dispostas em mais de uma província geocronológica, como é o caso das províncias minerais de Tapajós e Alta Floresta. A Província Mineral do Tapajós localiza-se entre as províncias geocronológicas VentuariTapajós e Amazônia Central de Tassinari \& Macambira (op. cit.) ou de Tapajós-Parima e Amazônia Central de Santos et al. (2008).

A Província Aurífera de Alta Floresta (PAAF), que está localizada no centro-sul do Cráton Amazônico, no extremo norte do estado de Mato Grosso (Almeida, 1978; Almeida et al. 1981) e é o local onde ocorre o presente estudo, de acordo com a proposta de divisão de Santos et al. (2008) é compreendida entre as províncias geocronológicas Tapajós - Parima (2,1-1,87 Ga) e Rondônia - Juruena (1,82-1,54 Ga), 
ou de Tassinari \& Macambira (1999) entre Ventuari-Tapajós - PVT (1,95-1,80 Ga) e Rio Negro-Juruena - PRNJ (1.8-1.55 Ga).

A Província, foi sub-dividida por Santos (2003) em quatro domínios principais: Jamari, Alta Floresta, Juruena e Alto Jauru. Lacerda Filho et al. (2004) redefiniram a Província Rondônia - Juruena, reposicionando-a no intervalo de 1,85 - 1,75 Ga e definindo a existência de três domínios: Juruena $(1,85-1,75 \mathrm{Ga})$, Roosevelt-Aripuanã $(1,76$ - 1,74 Ga) e Jauru $(1,79$ - 1,72), tais autores excluíram o Domínio Jamari dessa província. Santos (2003) propõe cinco arcos magmáticos na Província RJ: Arco colisional V (Quatro Cachoeiras 1670 - $1630 \mathrm{Ma}$ ); arco continental IV (1740 Ma; Roosevelt; arco de ilhas e continental Jamari e Jauru 1740 - 1760 Ma); Arco Magmático Continental III (1770 - 1785 Ma, incluindo o grupo Colíder, São Romão, Monte Verde e Vitória); Arco Magmático II (1800 - 1820 Ma; membros: Paranaíta. Cristalino, Juruena e Vulcânicas do Moriru), Arco de Ilhas I (Bacaéri com idades mais antigas que $2,0 \mathrm{Ga}$ ).

De acordo com Lacerda Filho et al. (2004) o Domínio Juruena é constituído por rochas plutônicas e vulcânicas félsicas de arco tipo Andino, de filiação cálcico-alcalina de alto potássio, formadas entre 1760 e 1820 Ma. Nesse arco magmático Juruena (1,85 - 1,75 Ga), Lacerda Filho et al. (2004) e Souza et al. (2005) descreveram dois terrenos, um acrescionário, deformado em regime dúctil, de médio a alto grau metamórfico e outro plutonovulcânico, pós-colisional, sin a tardi-orogênico. Segundo esses autores, o terreno acrescionário é representado pelas unidades denominadas Complexos Nova Monte Verde e Bacaeri-Mogno. Entretanto, Santos (2003) sugere um desmembramento do então chamado Complexo Bacaeri - Mogno, visto que reconhece charnockitos com características de rochas pós-tectônicas na porção Mogno, tonalitos, dioritos e basaltos, com características de arco juvenil e associados à porção Bacaeri, turbiditos metamorfizados na fácies anfibolito.

Segundo Silva \& Abram (2008), a evolução tectônica para a Província Aurífera de Alta Floresta, foi estabelecida em sete fases. A primeira com geração de um conjunto de rochas formada em um ambiente de tectônica extensional (Complexo BacaeriMogno); a segunda formada em ambiente de arcos magmáticos Cuiú-Cuiú e Juruena, corresponde a rochas de afinidades cálcio-alcalinas de margem continental ativa; a terceira fase é colisional representada pelo Granito Apiacás (1784 $\pm 32 \mathrm{Ma}$ ) com uma idade herdada de $(1871 \pm 21 \mathrm{Ma})$; a quarta fase configura delaminação crustal, com ocorrência de magmatismo intraplaca (Flor da Serra); na quita fase, formação de bacia 
intra-arco na qual foi gerada a sequência vulcano-sedimentar do Grupo São MarceloCabeça; fechamento do orógeno acontece na sexta fase, acompanhado de progressiva deformação e geração de mega estruturas de cisalhamento transcorrente; finalizando com a sétima fase, tardia, com a geração do Granito Teles Pires e Suíte Nova Canaã.

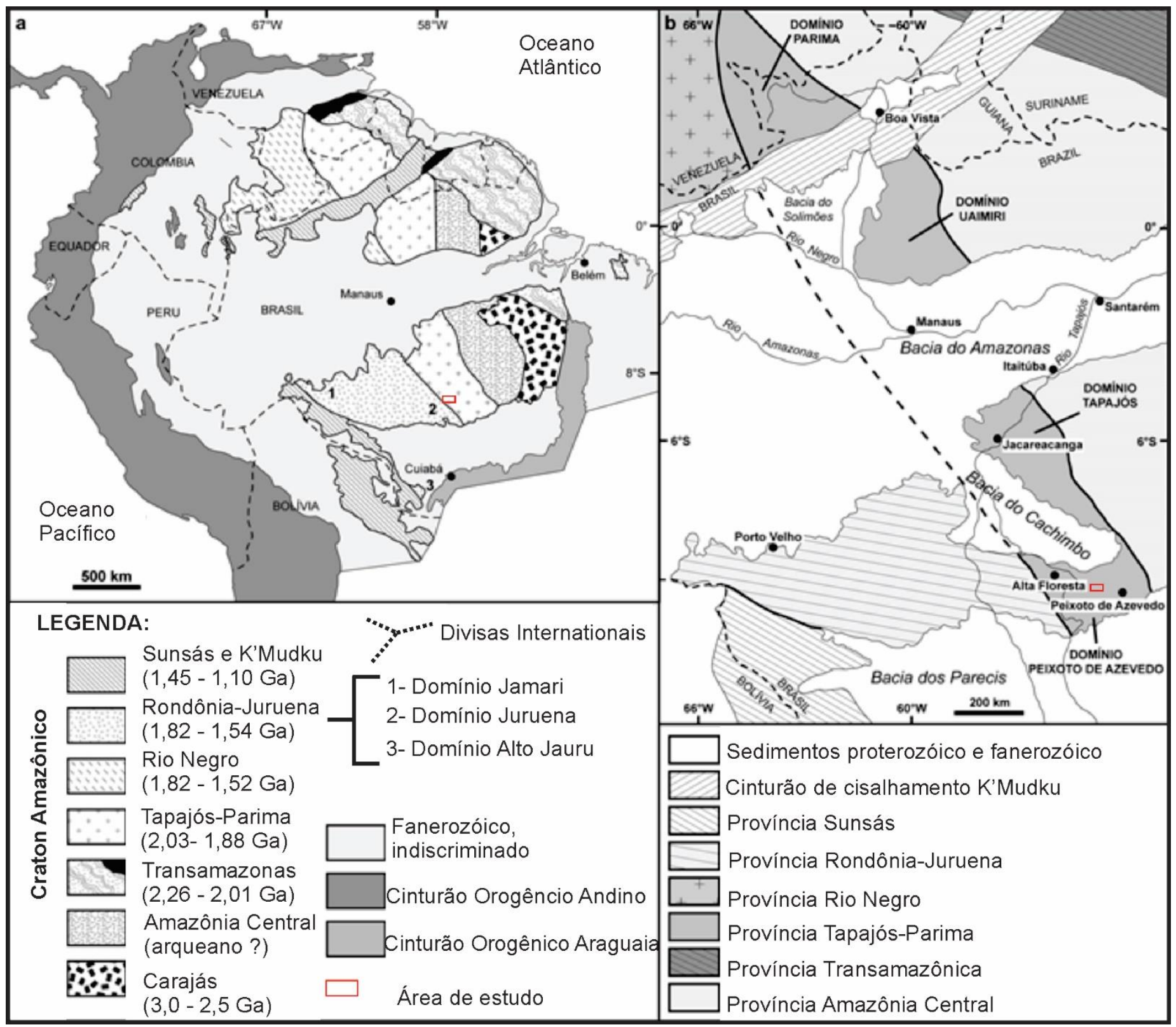

Figura 02 - Mapa Geotectônico do Cráton Amazônico. (A) Modelo geotectônico do Cráton Amazônico de Santos et al. (2004); (B) Subdivisão da Província TapajósParima em diferentes domínios metalogenético, Santos et al. (2008). 


\section{II.2 - GEOLOGIA REGIONAL}

A área de estudo, porção oeste da Província Aurífera de Alta Floresta é constituída pelas unidades: Embasamento arqueano; Complexo Cuiú-Cuiú; Suíte Intrusiva Nhandu; Suíte Intrusiva Matupá, Suíte Intrusiva Juruena, Grupo Colíder, Suíte Vulcano-Plutônica Teles Pires e coberturas sedimentares (Fig. 02).

O embasamento arqueano foi descrito por Paes de Barros (2007) para o Gnaisse Gavião, que ocorre nas proximidades da cidade de Peixoto de Azevedo. Entretanto, as idades arqueanas referem-se a um único afloramento, de dimensões muito restritas, podendo na verdade, evidenciar um embasamento heterogêneo com presença de crosta arqueana. Santos et al. (2015) reapresentaram dados U-Pb em zircão e em titanita para este afloramento. As idades obtidas para os cristais de zircão mostram duas famílias de 2,8Ga e 2,6 Ga, enquanto idades de titanita são de $1870 \mathrm{Ma}$, que podem representar idades de metamorfismo do embasamento arqueano. O Complexo Cuiú-Cuiú descrito por Pessoa et al. (1997) no Domínio Tapajós, foi mais tarde estendido para os domínios geográficos de Alta Floresta, Souza et al. (2005). Constitui-se de ortognaisses de composição monzonítica, tonalítica e granítica. Ocorrem na forma de corpos menores na forma de faixas estreitas alongadas descontinuas e esparsos, como as manifestações magmáticas mais antigas na região. Klein et al. (2000) descreve essa unidade como típica de ambiente de arco vulcânico. Datações em ortognaisse pelo método U-Pb em SHRIMP (Santos, 1999), revelaram idade convencional U-Pb entre $2005 \pm 7 \mathrm{Ma}$, realizada em gnaisse e granitóide. Dezula (2016) encontrou o mesmo resultado para gnaisses tonalíticos $(2008 \pm 3 \mathrm{Ma})$ que ocorrem a sul de Novo Mundo, nas proximidades do garimpo Aragão.

O granito Nhandu foi introduzido por Souza et al. (1979) para se referir as rochas graníticas porfiroblásticas de composição granodiorítica a tonalítica, com estrutura maciça e textura pseudo-rapakivi. Lacerda Filho (2001; 2004) caracteriza-o como de afinidade cálcio-alcalina de alto potássio, do tipo I oxidado, representado por magnetita-biotita granito de cor vermelha. Chamado inicialmente de Granito Nhandu, o termo foi mais tarde elevado à categoria de suíte por Moreton \& Martins (2003; 2005). Datações U-Pb em zircão por SHRIMP, determinou um intervalo temporal entre $1962 \pm$ 6 Ma e $1967 \pm 7$ Ma para a Suíte Intrusiva Nhandu, correlacionando-a com a Suíte Intrusiva Creporizão, dessa forma extendendo o Domínio Tapajós para o norte da Província Aurífera de Alta Floresta (Barros et al. 2015). 
A Suíte Intrusiva Matupá, termo inicialmente adotado por Moura et al. (1998) para individualizar um corpo de biotita monzogranito isotrópico que ocorre a sul da cidade de Matupá, consiste de um magmatismo oxidado, cálcico-alcalino de alto K, magnesiano e peraluminoso a ligeiramente metaluminoso. Os corpos mapeados como Granito Matupá (Paes de Barros 1994, 2007) ocorrem de forma alongada, na direção NW-SE na região do Depósito Serrinha. O granito Matupá na região de Serrinha (Moura et al. 1998) aflora como blocos não deformados e pouco fraturados, possui mineralizações de ouro em veio de quartzo e do tipo pórfiro (disseminado/stockwork) que são associados a uma importante fase hidrotermal. Uma idade $\mathrm{Pb}-\mathrm{Pb}$ em zircão de $1.872 \pm 12 \mathrm{Ma}$ foi obtida, além de idades modelo $\left(\mathrm{T}_{\mathrm{DM}}\right)$ que variam no intervalo 2,342,47 Ga, e $\varepsilon N d(\mathrm{t})$ variando de $-2,7$ a $-4,3$ (Moura et al. 1998). Silva \& Abram (2008) obtiveram idade modelo $\mathrm{Nd} \mathrm{T}_{\mathrm{DM}}$ de $2,15 \mathrm{Ga}$ com $\varepsilon \mathrm{Nd}(\mathrm{t}=1.87 \mathrm{Ga})$ de $-0,98$. Recentes datações U-Pb (SHRIMP) realizadas por Silva et al. (2014), mostraram que uma fácies de biotita monzogranito, anteriormente definida como pertencente ao Granito Peixoto, apresenta uma idade de $1869 \pm 10$ Ma, similar à Suíte Intrusiva Matupá, de biotita monzogranito.

De acordo com Silva et al. (1974) a Suíte Intrusiva Juruena é constituída por um conjunto de rochas graníticas, alinhadas segundo um trend NW-SE, frequentemente gnaissificados e elipsoidais. Dados geocronológicos usando U-Pb em zircão demonstram idade de $1817 \pm 12 \mathrm{Ma}$ (Silva \& Abram, 2008). Geoquimicamente as rochas que compõem essa suíte são de afinidade cálcioalcalinas, com alto potássio, metaluminosas a peraluminosas, não deformadas e não magnéticas, padrões enriquecidos em ETRL e com ETRP levemente fracionadas, com características semelhantes às rochas graníticas de arco magmático de margem continental ativa (Souza et al. 2004). Neste trabalho, considera-se a Suíte Intrusiva Paranaíta como parte da Suíte Intrusiva Juruena, com base nos dados isotópicos de Silva \& Abram (2008) que apresentam idades U-Pb em zircão para as rochas da Suíte Paranaíta de $1808 \pm 14 \mathrm{Ma}$.

O Grupo Colíder é representado por vulcânicas (efusivas e piroclásticas) de composição intermediária a ácida, de alto potássio, cálcio-alcalina, peraluminosa a metaluminosa e exibem afinidade geoquímica com as séries graníticas orogênicas (Moreton \& Martins 2005).

As vulcânicas do Grupo Colíder apresentam contatos tectônicos com as Suítes Matupá e Nhandu, além de possuírem idades U-Pb em zircão de $1781 \pm 8$ Ma e $1785 \pm$ 6,3 Ma, e idade modelo $\mathrm{T}_{\mathrm{DM}}$ de 2,34 Ga e ENd(t) de -3,75 (Pimentel 2001; Silva \& 
Abram 2008). Rochas graníticas de idade similar as vulcânicas Colíder tem sido descritas como Granito São Pedro (Souza et al. 2005), Granito São Romão e Suíte Intrusiva Mogno (Barros et a.l 2016).

A Suíte Intrusiva Teles Pires é composta por rochas vulcânicas ácidas do sul da Serra do Cachimbo (Bini, et al. 2015), pelo granito Terra Nova (Prado et al. 2013; Barros et al. 2013), além das unidades já descritas como parte do magmatismo teles pires (Pinho et al. 2003) e Barros et al. (2009). A Suíte Intrusiva Teles Pires, inicialmente descrita por Souza et al. (2005), compreende rochas plutônicas e subvulcânicas, constituídas majoritariamente por álcali-feldspato granito e sienogranito, contendo subordinadamente monzogranito e granodioritos. Datação U-Pb em zircão definiu uma idade de $1.757 \pm 16 \mathrm{Ma}$ e idade $\mathrm{T}_{\mathrm{DM}}$ de $2100 \mathrm{Ma}$ (Santos, 2000). Baseado em dados isotópicos de U-Pb em zircão e valores de $\mathrm{T}_{\mathrm{DM}}$, Pinho et al. (2003) englobou o vulcanismo de caráter bimodal da região de Moriru (norte de Aripuanã) ao Magmatismo Teles Pires, rochas vulcânicas félsicas com idades U-Pb (TIMs) entre $1770 \pm 6$ Ma a $1778 \pm 6$ Ma e idades modelo $\mathrm{Nd}_{\mathrm{DM}}$ de 2,0 a 2,1 Ga. Bini et al. (2015) datou riolitos do sul da Serra do Cachimbo por U-Pb em SHRIMP e obteve $1757 \pm 14$ Ma. Esta última idade é considerada a mais próxima do esperado para esse magmatismo pós-colisional a anorogênico. (Figuras 04 e 05) 


\section{CAPÍTULO II - GEOLOGIA REGIONAL E CONTEXTO GEOTECTÔNICO}

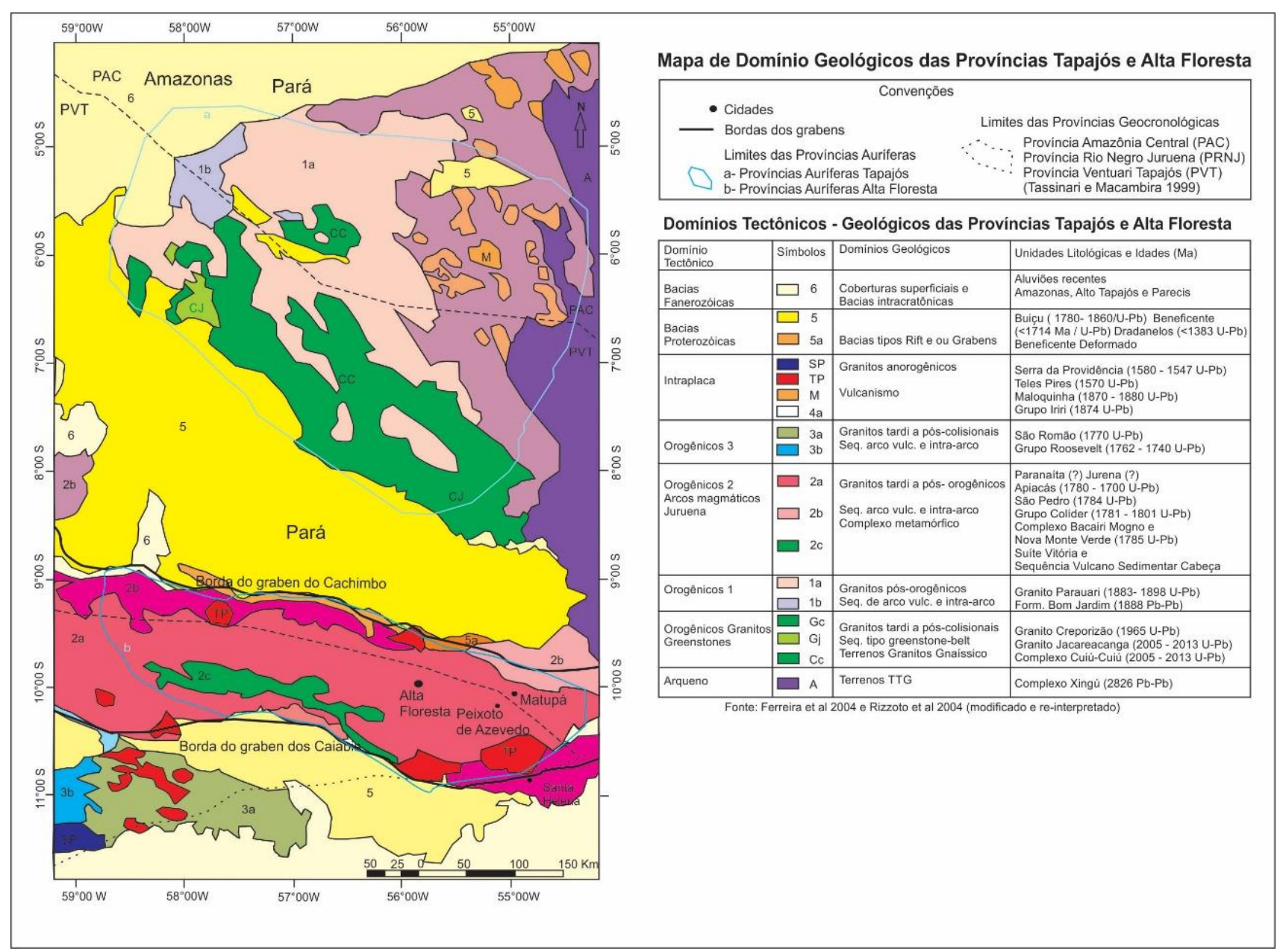

Figura 03 - Mapa Geológico das Províncias Auríferas de Alta Floresta e Tapajós, limitadas por bacias sedimentares ao norte e sul. (extraído de Paes de Barros, 2007). Na legenda encontam-se os principais domínios tectônicos e seus repectivos domínios geológicos. 


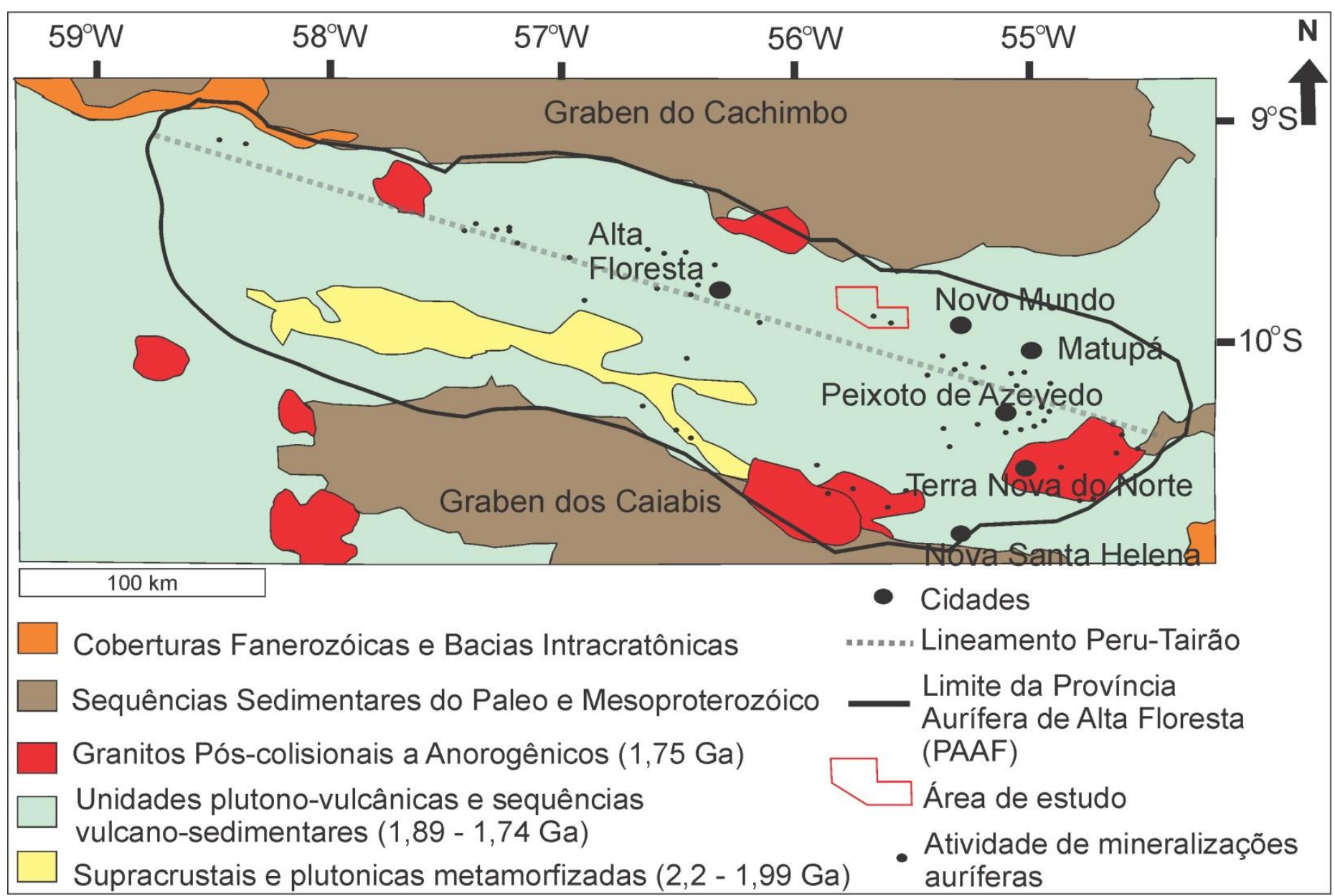

Figura 04 - Mapa Geológico da região da Província Aurífera de Alta Floresta. Adaptado de Lacerda Filho et. al. (2004), Souza et. al. (2005), Vasques \& Rosa-Costa (2008), Silva \& Abram (2008) e Alves et. al. (2010). Destaca-se que os locais de ocorrência de depósitos de ouro estão alinhados N8OW-S80E, segundo lineamento Peru-Trairão

(LPT). 


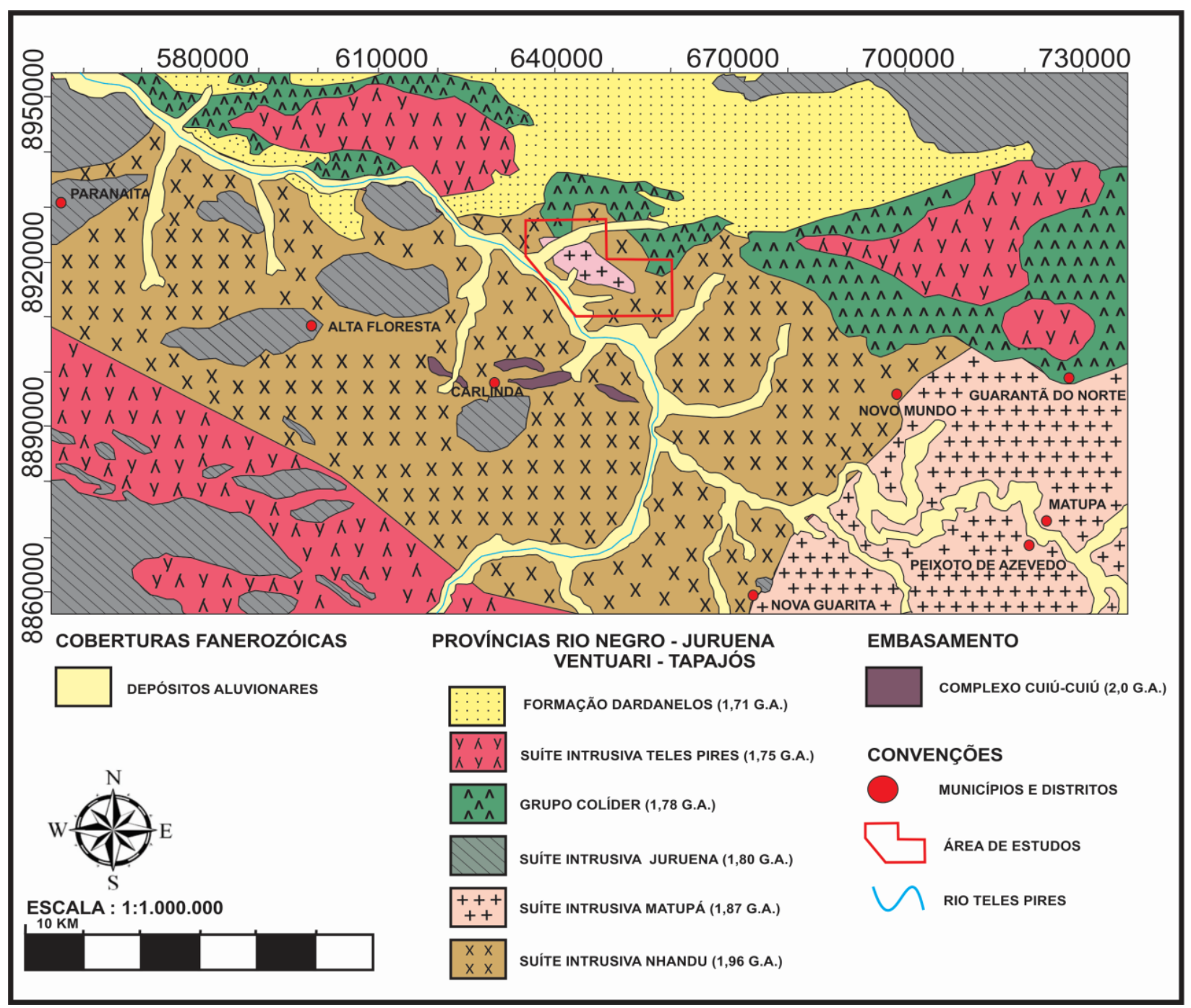

Figura 05 - Mapa geológico regional da parte leste da PAAF, com localização da área de pesquisa, modificado de Miguel Jr. 2011. 
CAPÍTULO III - GEOLOGIA LOCAL

\section{CAPÍTULO - III}

GEOLOGIA LOCAL 


\section{III.1 - GEOLOGIA LOCAL}

Os corpos mapeados apresentam dimensões da ordem de $80 \mathrm{Km}^{2}$, aflorantes em blocos e matacões, se constituindo em colinas suaves, os mehores afloramentos estão lozalizados na margem direita do Rio Teles Pires. Em campo, as rochas foram divididas em duas fácies, uma fácies de granulação fina a média (biotita sienogranito) e outra média a levemente porfirítica (biotita monzogranito). Ambas apresentam cor rosa médio a claro com tons avermelhados quando oxidados ou esbranquiçados quando argilizados. São constituídos essencialmente por feldspato potássico, quartzo, plagioclásio, com proporções variadas de minerais máficos, esses representados por biotita e/ou hornblenda. Como minerais acessórios são encontrados titanita, apatita. Como minerais secundários foram identificados epidoto, clorita, sericita e argilominerais. Entre os opacos, destaca-se a magnetita com ocorrência em praticamente toda unidade mapeada. Sulfetos estão presentes próximo a zonas mineralizadas associados a veios de quartzo e consistem principalmente de pirita e em menor proporção de calcopirita, esfalerita e ilmenita.

O biotita monzogranito preserva textura isotrópica, e apresenta localmente feições de textura primária de fluxo magmático. Corpo com textura inequigranular (cristais de até $3 \mathrm{~cm}$ ) a porfirítico, sendo que de modo restrito ocorre a textura pseudorapakivi. O biotita monzogranito situados ao norte contêm abundantes enclaves microgranulares de composição intermediária, granulação fina e formas arredondadas e algumas angulosas e subangulosas com dimensões de 10 a $50 \mathrm{~cm}$. Ocorrem na fácies de borda e de cúpula dos corpos de biotita monzogranito grosso, indicando intrusão por magmatic stoping. Os sulfetos e óxidos metálicos estão dispostos de forma disseminada na rocha em estruturas do tipo boxwork ou associados a veios de quartzo tardios. (Figura 07)

Obiotita sienogranito é cortado em sua porção central por diques microgranulares félsicos (diques de aplito) de coloração rosa-claro e de composição similar ao granito e granulação fina. Feições de campo mostram contatos nítidos do dique fino com a rocha hospedeira, apresentando formas geralmente tabulares a alongadas com espessura variando de $3-15 \mathrm{~cm}$, feições de estruturas de fluxo sugestivas de mistura de magma ocorrem localmente. (Figura 06) 
Evidências de campo sugerem que os dois granitos são formados em eventos próximos. Além dos diques félsicos foi observado diques máficos (diabásio) que cortam o granito em questão, com direção NW-SE e contato plano bem definido sem evidências de magma mingling. Ausência de contato metamórfico ou halos de alteração com as rochas adjacentes sugere resfriamento rápido para os diques, os quais mostram textura afaníticas a microporfiríticas, com fenocristais de plagioclásio de granulação fina a média.

Veios tardios de quartzo e epidoto cortam o corpo intrusivo em diferentes direções. As fraturas, associadas à zonas de cisalhamento, podem ser agrupadas em até três padrões distintos; F1 com direções variando em N85W-S80E; F2 com direção NE/SW; e F3 com direção W/E ocorrendo com menor frequência na área estudada.

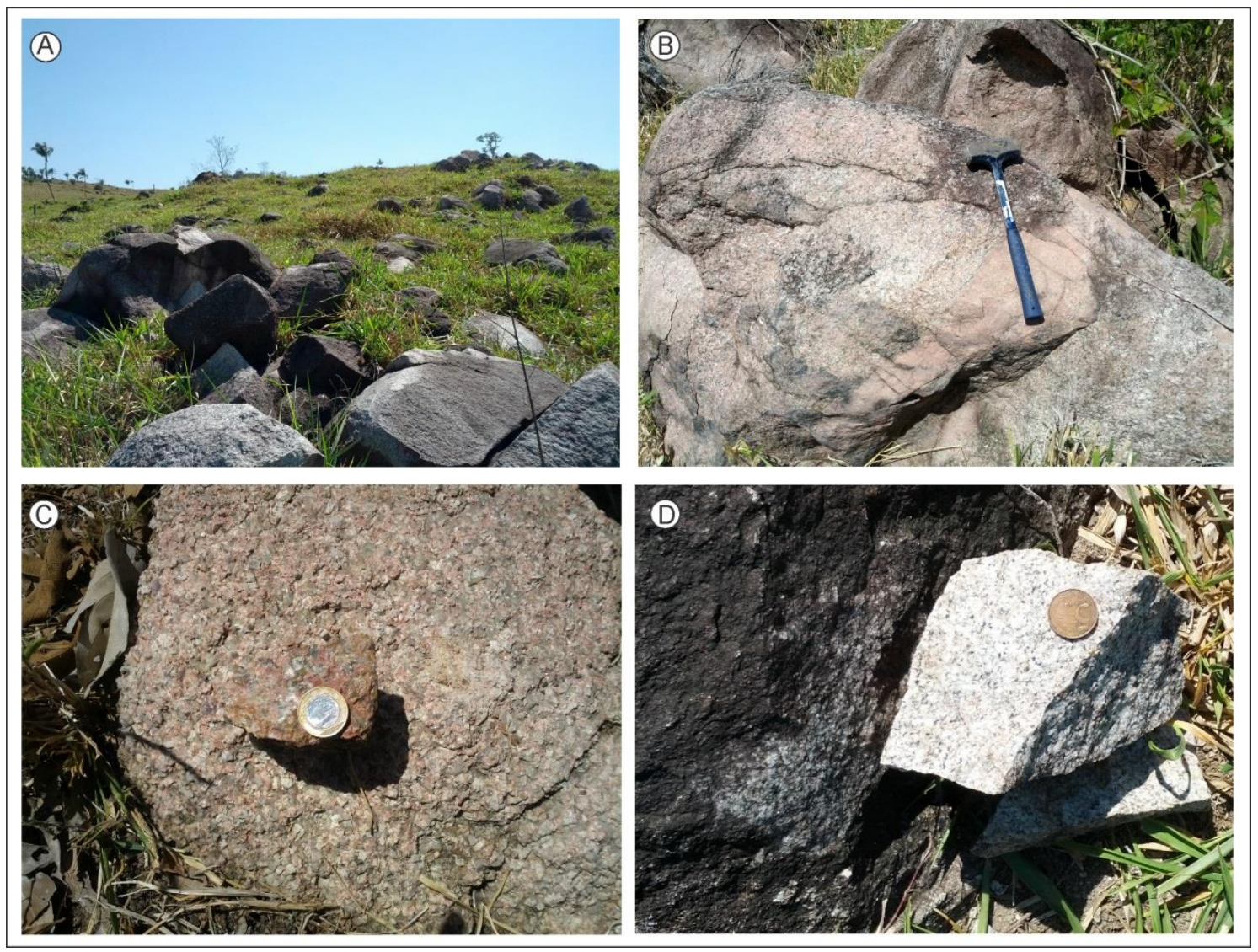

Figura 06 - A - Forma de ocorrência dos afloramentos, formando colina suave; B-

Dique de aplito cortando fácies de granulação média do biotita sienogranito; $C$ Granito rosado, granulação média; D- Detalhe do biotita monzogranito isotrópico. 


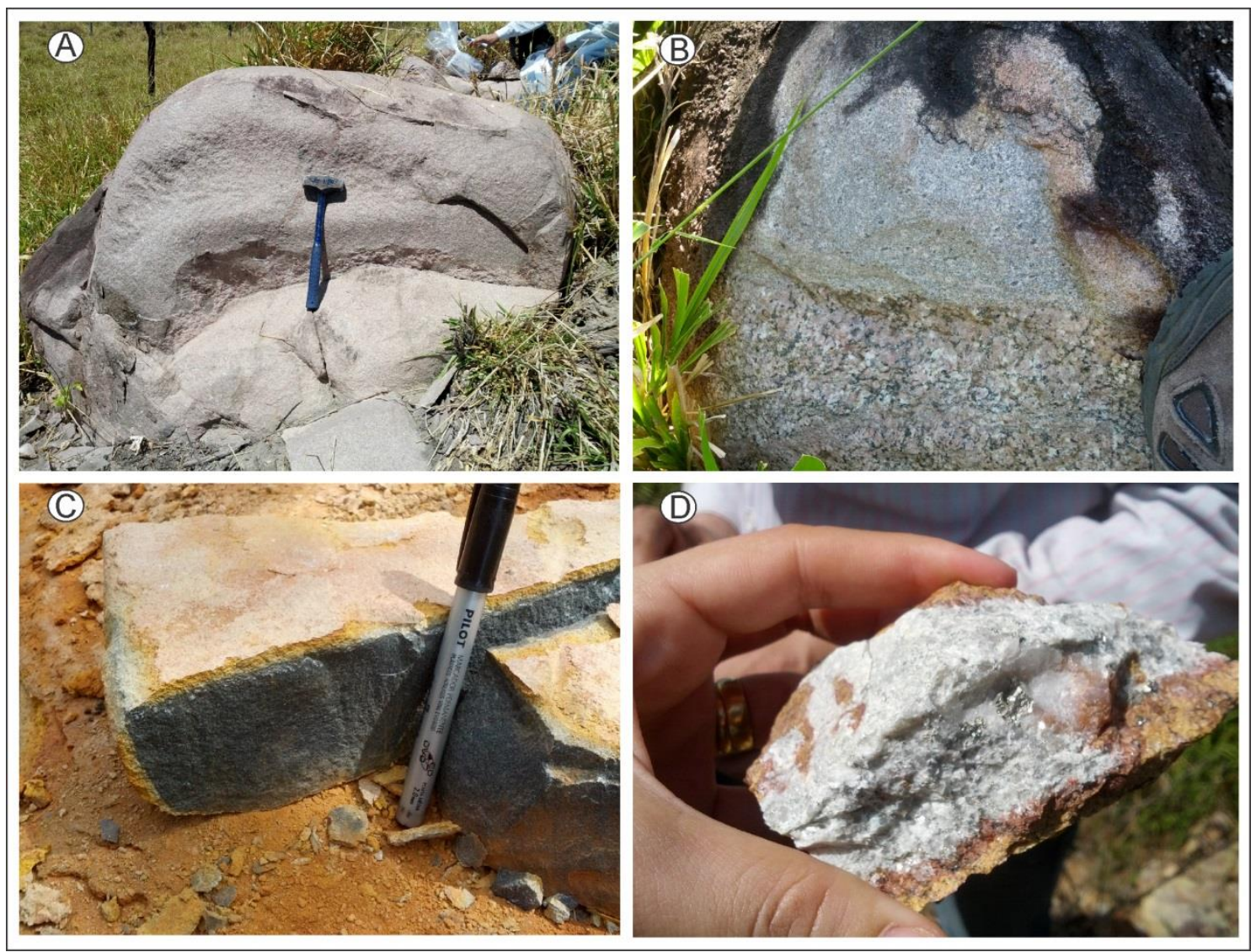

Figura 07 - A- Afloramento de biotita sienogranito de granulação fina a média; $B$ Enclaves de composição intermediária a ácida; C-Dique de diabásio; D- Sulfetos associados a veios de quartzo, próximo à zona mineralizada. 


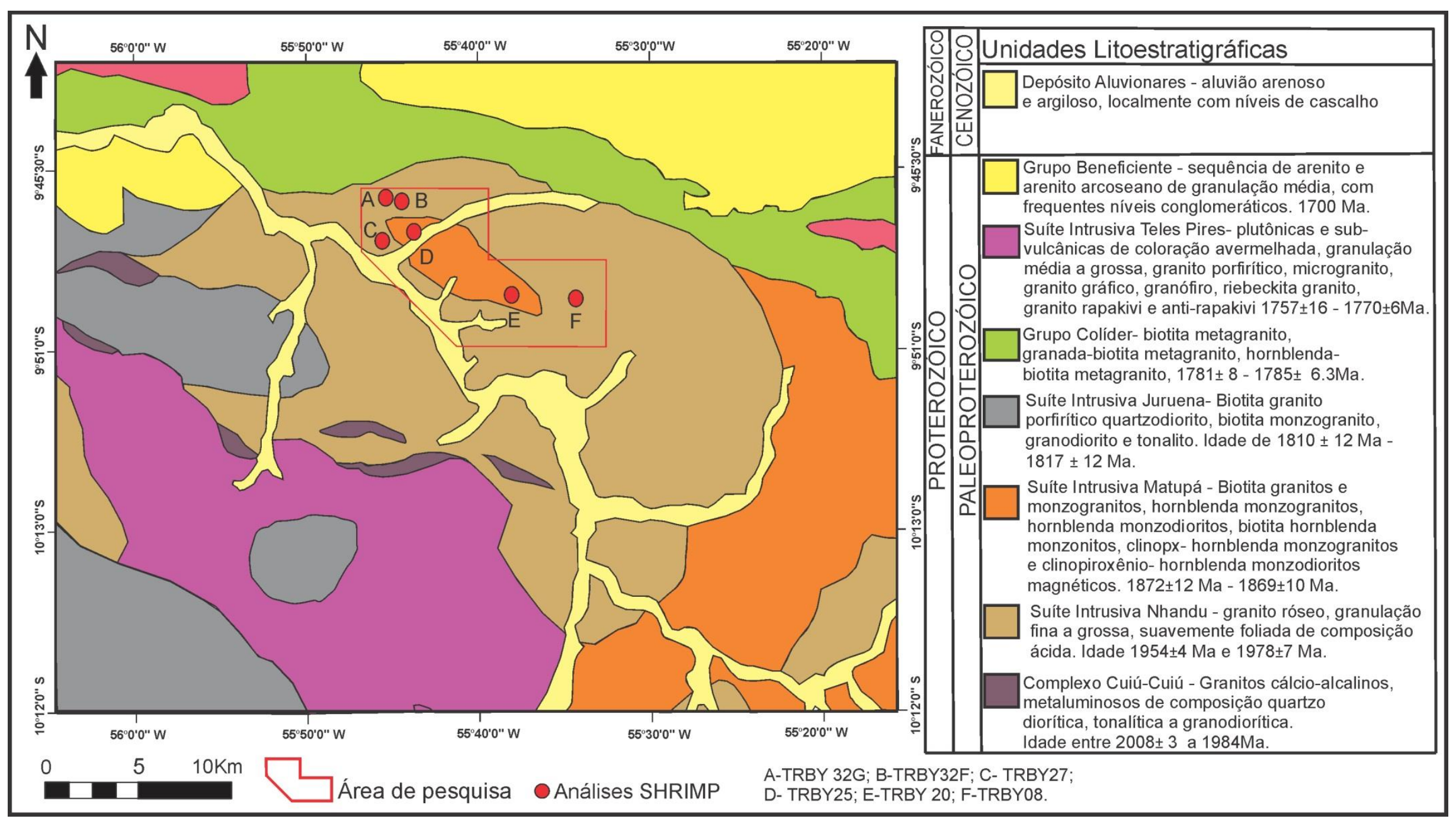

Figura 08 - Esboço geológico regional, modificado de Miguel Jr (2011), com a área de pesquisa. Dados U-Pb obtidos no presente trabalho. 


\section{III.2 - PETROGRAFIA}

\section{III.2.1 - Biotita Sienogranito}

Microscopicamente é uma rocha de granulação fina a média constituída por feldspato potássico, quartzo, plagioclásio e biotita. Sob o microscópio o biotita siogranito apresenta uma textura xenomórfica a hipidomórfica, sendo composto pelas seguintes proporções aproximadas de minerais: $45 \%$ de feldspato potássico, $25 \%$ de quartzo; $20 \%$ de plagioclásio e $10 \%$ de biotita + hornblenda + clorita + opacos, baseado em estimativa visual. Zircão, titanita, apatita e rutilo foram observados como acessórios geralmente inclusos em outros minerais. Clorita, epidoto e sericita ocorrem como minerais secundários. (Figura 09)

Os cristais de feldspato potássico, ocorre em prismas subédricos essencialmente pertítico, sendo as pertitas são do tipo strings. Geminação combinada albita + periclina (microclínio) exibe inclusões de dimensões variáveis $(0,5$ a $4 \mathrm{~mm})$, comumente com aspecto turvo devido à alteração para argilo-minerais e sericita. Localmente apresenta inclusões de minerais máficos.

Quartzo ocorre em agregados recristalizados com contatos serrilhados, com hábito anédrico e dimensões de 1 a $2 \mathrm{~mm}$, sendo que exibem fraca extinção ondulante, devido à recristalização sob tensão relacionado a zonas de cisalhamento, subgrãos e cristais fraturados com algumas feições de intercrescimento gráfico.

O plagioclásio, identificado como oligoclásio pelo método estatístico de Michel Levy (Kerr, 1959), ocorre como cristais tabulares a subédrios com tamanho de 1 a 1,5 $\mathrm{mm}$, com inclusões poiquilítica de opacos, clorita e/ou biotita. Geminados segunda a lei da albita. Comumente os cristais apresentam evidências de processos de moderada a intensa alteração, como sericitização e saussuritização com desenvolvimento de epidoto/clinozoizita, sericita e calcita

A biotita constitui a principal fase ferromagnesiana, de cor marrom escuro, pleocroísmo de marrom claro a marrom escuro. Ocorre geralmente em lamelas intersticiais ou em agregados com os outros minerais, com dimensões variando de 0,5 a $1,5 \mathrm{~mm}$ e pleocroísmo de amarelo-alaranjado a castanho-escuro. Geralmente representam a única fase máfica e estão alteradas para clorita.

A apatita corresponde a uma fase primária acessória, ocorrendo em minúsculos cristais aciculares inclusos em outros minerais principalmente no plagioclásio. Pequenos cristais esfenoidais de titanita de cor marrom avermelhada estão presentes. $\mathrm{O}$ 
zircão apresenta hábito euédrico, na forma de prismas bem desenvolvidos. A magnetita ocorre como cristais subédricos. Pequenos cristais de monazita, rutilo e barita, foram identificados com auxílio de EDS (Energy Dispersive System) em Microscópio Eletrônico de Varredura.

Os opacos ocorrem em pequena quantidade, podendo representar produtos de alteração dos minerais primários, com forma subédricas a anédricas, ocorrem disseminados por toda rocha e localmente, apresentam evidências de desopacitização com neoformação de biotita e clorita.

A clorita e epidoto ocorrem como produto de alteração na forma de agregados associado aos minerais opacos de hábito micáceo a acicular. Mica branca (fengita) oriunda de alteração secundária dos plagioclásios.

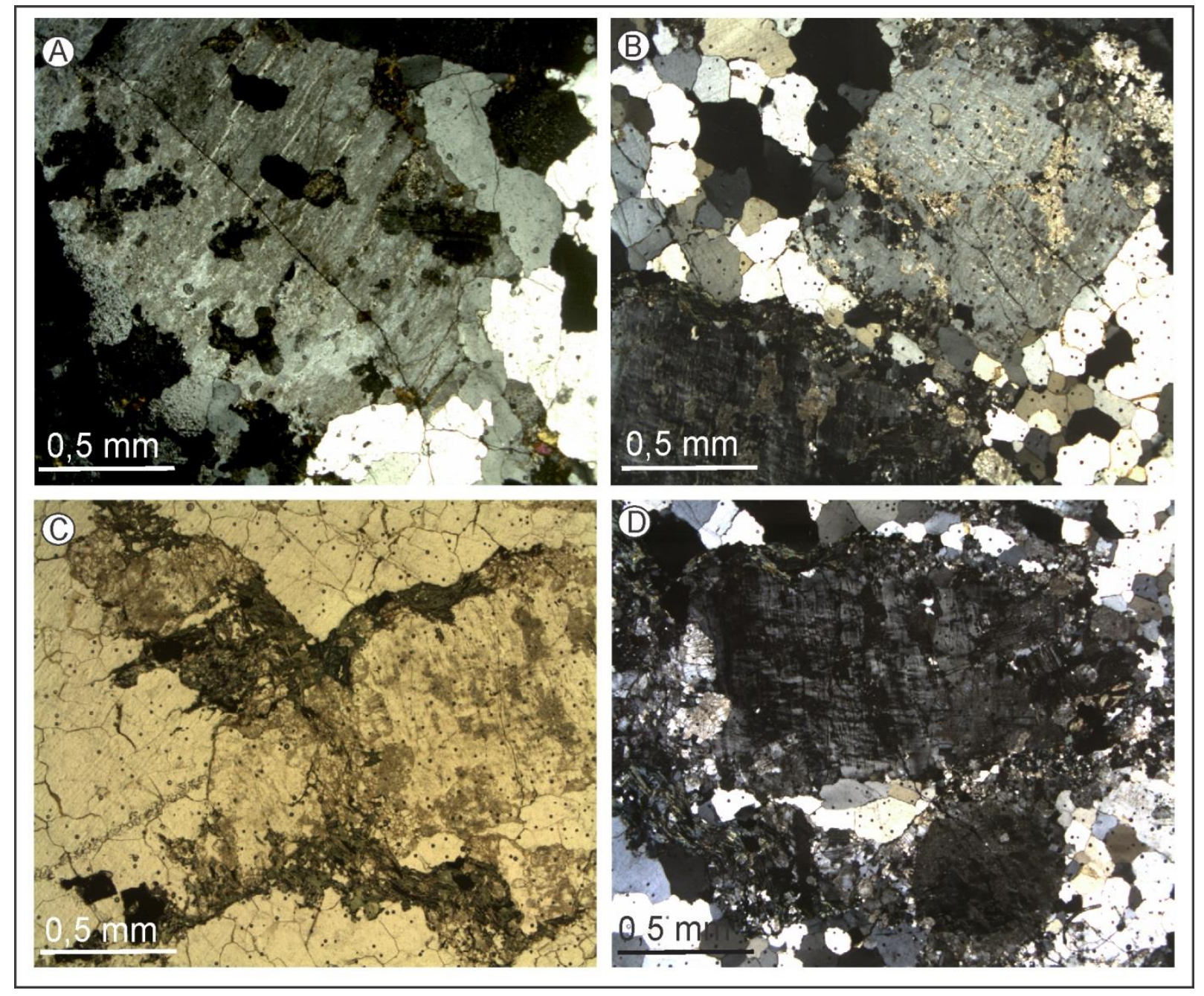

Figura 09- (A) fenocristal de feldspato potássico com intercrescimento pertítico (nicóis cruzados); (B) fenocristal de feldspato potássico com textura micropertítica em matriz fina composta por cristais de quartzo recristalizados (nicóis paralelos); (C) agregado 
de biotita, clorita secundária e minerias opacos (nicóiss paralelos); (D) fenocristal de microclínio com vestígio de geminação xadre em matriz composta por cristais de quartzo com contatos irregulares (nicóis paralelos).

\section{III.2.2 - Biotita Monzogranito}

Microscopicamente o biotita monzogranito é constituído por rochas holocristalinas de textura inequigranular com granulação média com pequeno conteúdo de minerais máficos. A assembleia mineralógica principal é constituída por plagioclásio (35\%), quartzo (35\%), feldspato alcalino (25\%) e biotita + hornblenda (5\%). Contato entre os cristais é dominantemente côncavos-convexos, por vezes com padrões irregulares. Os minerais acessórios são representados por rutilo, apatita, zircão e pequenas proporções de magnetita e assembleia mineral secundária composta por sericita, fengita, epidoto e clorita.

Plagioclásio $\left(\mathrm{An}_{10-30}\right)$, estimado pelo método estatístico de Michel Levy (Kerr, 1959), de hábito subédrico com maclas polissintéticas (segundo a lei da albita), mede de 0,5 até $1,5 \mathrm{~mm}$. Presença de cristais zonados e intensamente sericitizados e saussuritizados é comum para tais rochas. Localmente, foram observadas pequenas inclusões de minerais acessórios no núcleo do cristal (textura poiquilítica). (Figura 10)

O quartzo apresenta-se contato serrilhado com os outros minerais. Os grãos medem de $0,2 \mathrm{~mm}$ a $1,5 \mathrm{~mm}$, sendo que os menores grãos ocorrem nas porções mais recristalizadas formando subgrãos. Apresenta também feições de intercrescimento gráfico com o feldspato alcalino.

O feldspato potássico, com dimensões de 0,5 a $1,0 \mathrm{~mm}$, ocorre de forma anédrica a subédrica, representados por microclínio com geminação em grade (combinada albita + periclina), e também ortoclásio, com intercrescimento de micro-pertítico tipo filete. Comumente os cristais de feldspato potássico contém inúmeras inclusões de biotita, opacos e apatita, o que lhes conferem um aspecto poiquilítico.

A biotita representa a principal fase máfica dessas rochas, possui cor marrom, hábito lamelar (até $0,4 \mathrm{~mm}$ ) e ocorre principalmente nos interstícios dos demais minerais. Frequentemente a biotita está parcial ou totalmente transformada em clorita e minerais opacos. 
Hornblenda é um mineral varietal no corpo estudado com dimensões de 0,5. Quando aparece mostra hábito euedral a subedral, ocorre associada a diminutas lamelas de biotita e titanita magmática, com pleocroismo de verde claro a verde escuro.

A apatita ocorre como cristais idiomórficos, em pequenas agulhas. $\mathrm{O}$ zircão ocorre como cristais idiomórficos de tamanhos variados (menores que 0,01mm). A magnetita e rutilo ocorren em pequenas proporções, como cristais anédricos associados aos minerais máficos.

Os minerais opacos geralmente ocorrem como inclusões associado a biotita e hornblenda, com dimensões de até $0,5 \mathrm{~mm}$, são euédricos a subédricos exibindo contato serrilhados e, às vezes, retilíneos com os outros minerais.

Como minerais de alteração ocorrem sericita, fengita, epidoto e clorita, de forma lamelar, crescendo principalmente nos planos das maclas dos plagioclásios, no núcleo dos feldspatos potássicos e ou formando agregados. Clorita ocorre como pequenas lamelas pouco desenvolvidas preenchendo interstícios, com tamanhos de 0,4 mm.

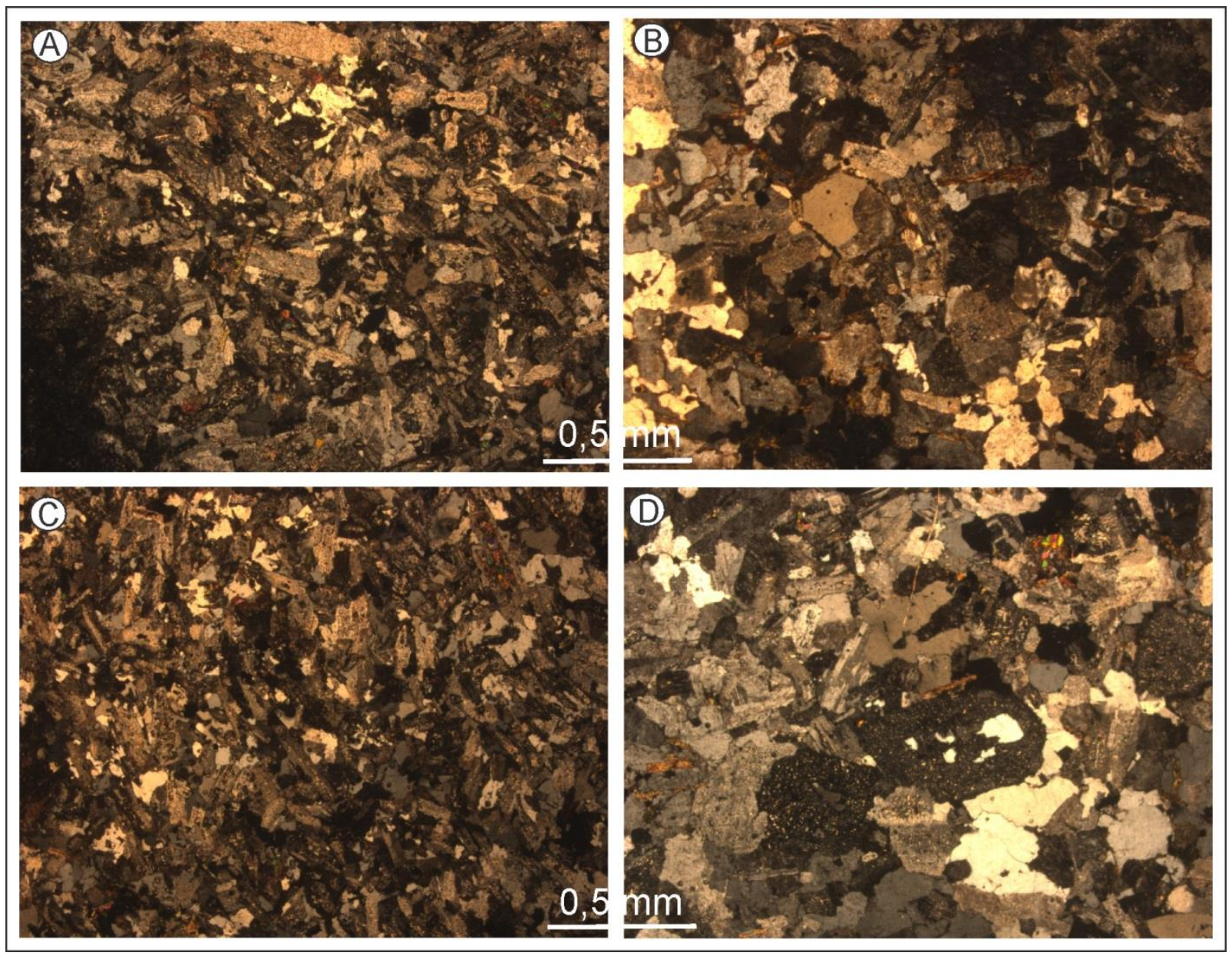


Figura 10- Rocha com textura ígnea granular com cristais de plagioclásio (oligoclásio), feldspato potássico com aspecto turvo, quartzo e biotita intensamente alterada para clorita; $(B, C)$ Cristais de plagioclásio com geminação polissintética $e$ saussuritizados, biotita intersticial e epidoto secundário; (D) cristais de plagioclásio saussuritizados com múltiplas geminações e com inclusões, textura poiquilítica. (todas as fotomicrografias em nicóis cruzados).

\section{III.2.3 - Enclaves Microgranulares Félsicos a Intermediários}

Enclaves microgranulares de composição félsica a intermediária (quartzo monzonítica) ocorrem no biotita monzogranito. Apresentam granulação fina e são compostos essencialmente por $60 \%$ de plagioclásio, $20 \%$ de quartzo, $15 \%$ de feldspato potássico e 5\% de anfibólio e minerais acessórios. Feldspato potássico (1 a 2,0 mm) comumente apresenta aspecto poiquilítico com inclusões de sericita e minerais opacos. O plagioclásio ocorre com geminação polissintética com tamanhos de até 1,5 $\mathrm{mm}$. Quartzo granular ou em subgrãos com leve extinção ondulante preenchem os interstícios entre os minerais precoces. Anfibólio (identificado por EDS como hornblenda rica em $\mathrm{Fe}$ ) apresenta dimensões de até $1,5 \mathrm{~mm}$, sendo comum feições de golfos de corrosão com pleocroísmo verde claro a verde escuro. Apatita, como fase primária acessória, em pequenos cristais aciculares inclusos principalmente nos plagioclásios e anfibólios. (Figura 11)

Outros minerais primários comuns são: apatita, magnetita, ilmenita e titanita. Argilominerais e clorita ocorrem como minerais secundários, provavelmente relacionados com alteração do anfibólio e plagioclásio.

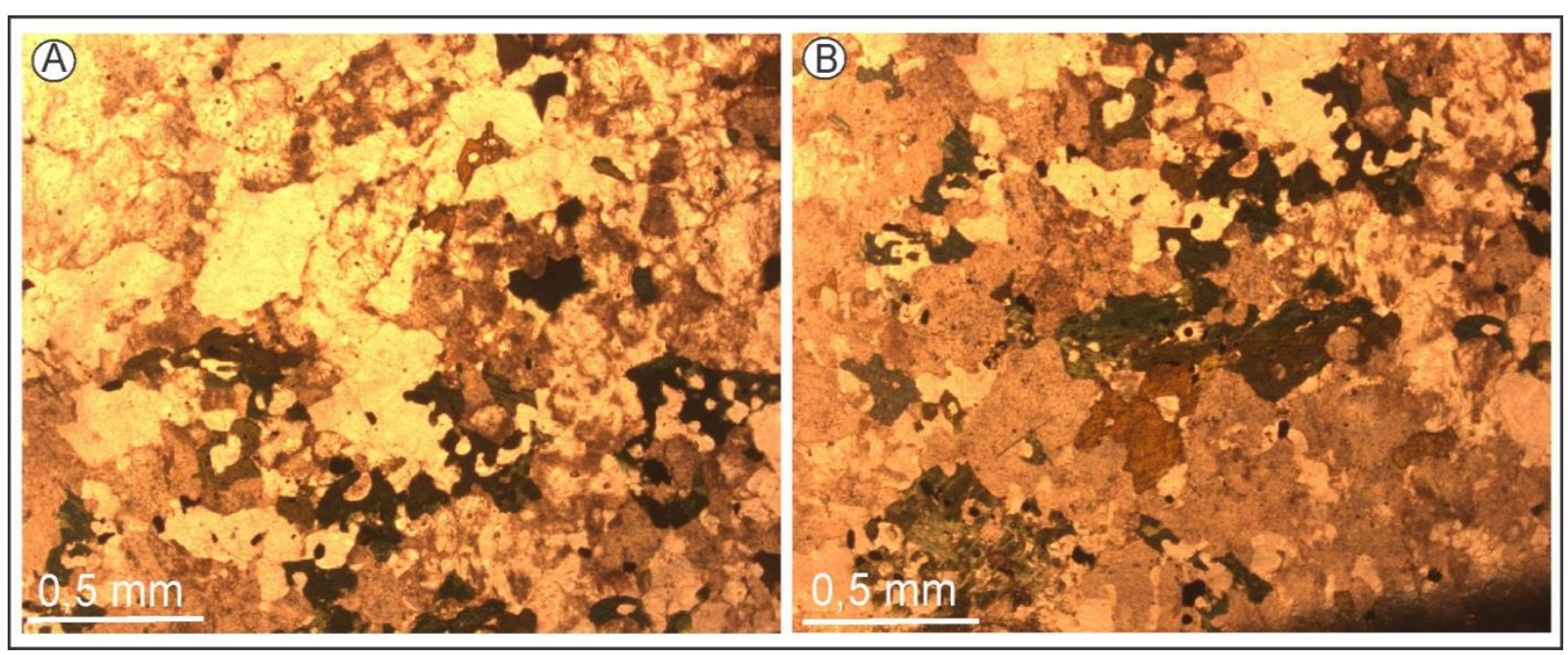


Figura $11-(A, B)$; cristal sub-édrico de hornblenda com bordas corroídas (nicóis paralelos)

\section{III.2.4 - Diques Máficos}

Diques de diabásio ocorrem com textura sub-ofítica a ofítica, holocristalino com granulação fina, por vezes com fenocristais de plagioclásio. Composto mineralógicamente segundo estimativa visual por plagioclásio $(50 \%)$, ortopiroxênio e clinopiroxênio (25 - $30 \%)$, minerais opacos (10\%), minerais secundários (5\%), e quartzo (4\%). (Figura 12)

Os cristais ripiformes de plagiocásio são prismáticos a subédricos, cujo tamanho varia de 0,1 a 1,3mm. Utilizando o método estatístico Michel Levy foram identificados como andesina $\left(\mathrm{An}_{30-50}\right)$, os cristais são zonados e com macla segundo lei da albita, raros fenocristais são observados. É comum evidências de processos de saussuritização nos plagiocásios.

Os cristais de piroxênio, identificados como augita são em geral subédricos a anédricos, com dimensões variando de 0,1 até $0,8 \mathrm{~mm}$, coloração verde-clara com pleocroísmo em tons de rosa, muitas vezes são incluídos parcialmente pelos cristais de plagioclásio, ocorrem como agregados associados aos minerais opacos. Comumente os grãos de piroxênios estão fraturados às vezes apresentam geminação setorial e/ou zonação.

Os feldspatos alcalinos ocorrem em pequena percentagem. Quartzo é pouco representativo e ocorre nos interstícios dos minerais precoces com tamanhos de 0,1 a $0,7 \mathrm{~mm}$.

Minerais opacos com hábito cúbico a subédrico, ocorrem em duas gerações como minerais primários e como alteração dos minerais máficos. Minerais acessórios perfazem 5\% da rocha, representados por pequenos grãos de zircão, badeleíta, magnetita, ilmenita, apatita e sulfetos (calcopirita), alguns desses minerais acessórios foram identificados com auxílio de EDS. Minerais de alteração pós-magmática, identificados como calcita, clorita, argilo-minerais e anfibólio produto de alteração dos piroxênios. 


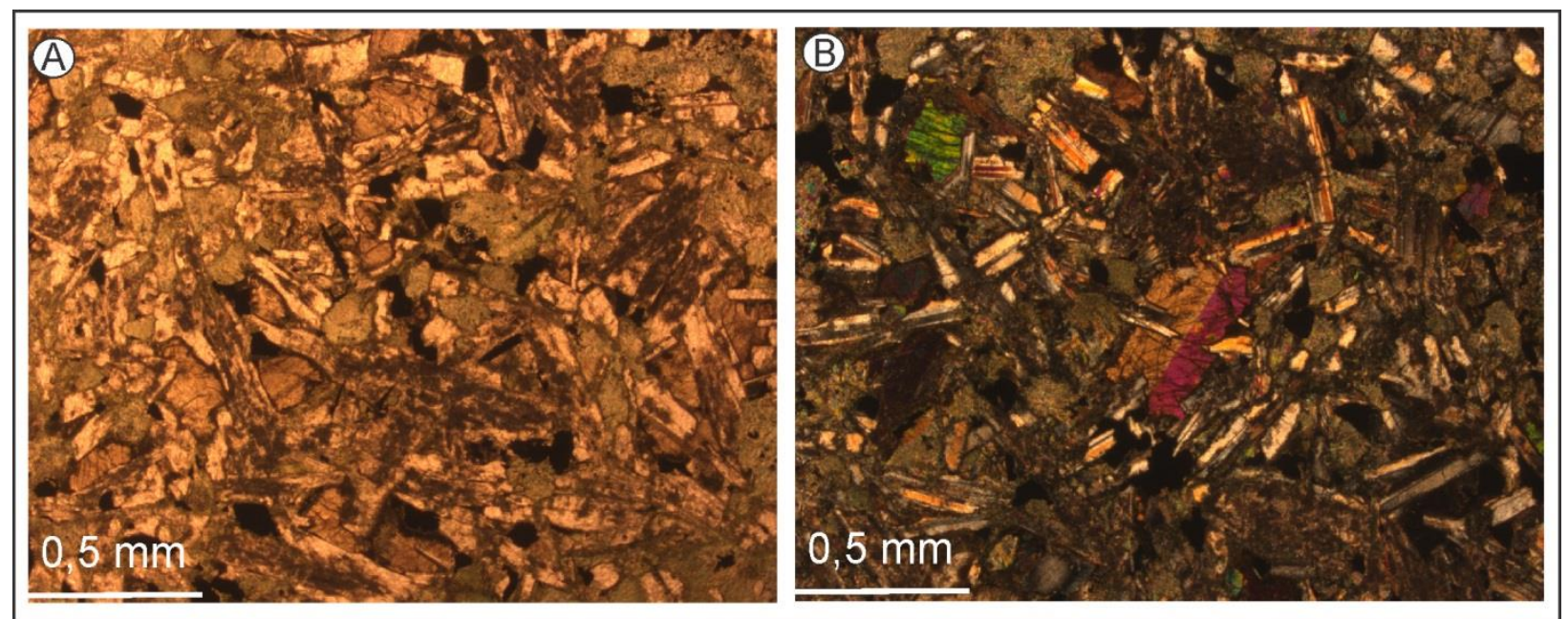

Figura 12- A - fotomicrografia de dique de diábasio com textura sub-ofítica, cristais tabulares de plagioclásio geminados (nicóis paralelos); B-clinopiroxênico (augita) com macla simples, plagioclásio e minerais opacos (nicóis cruzados).
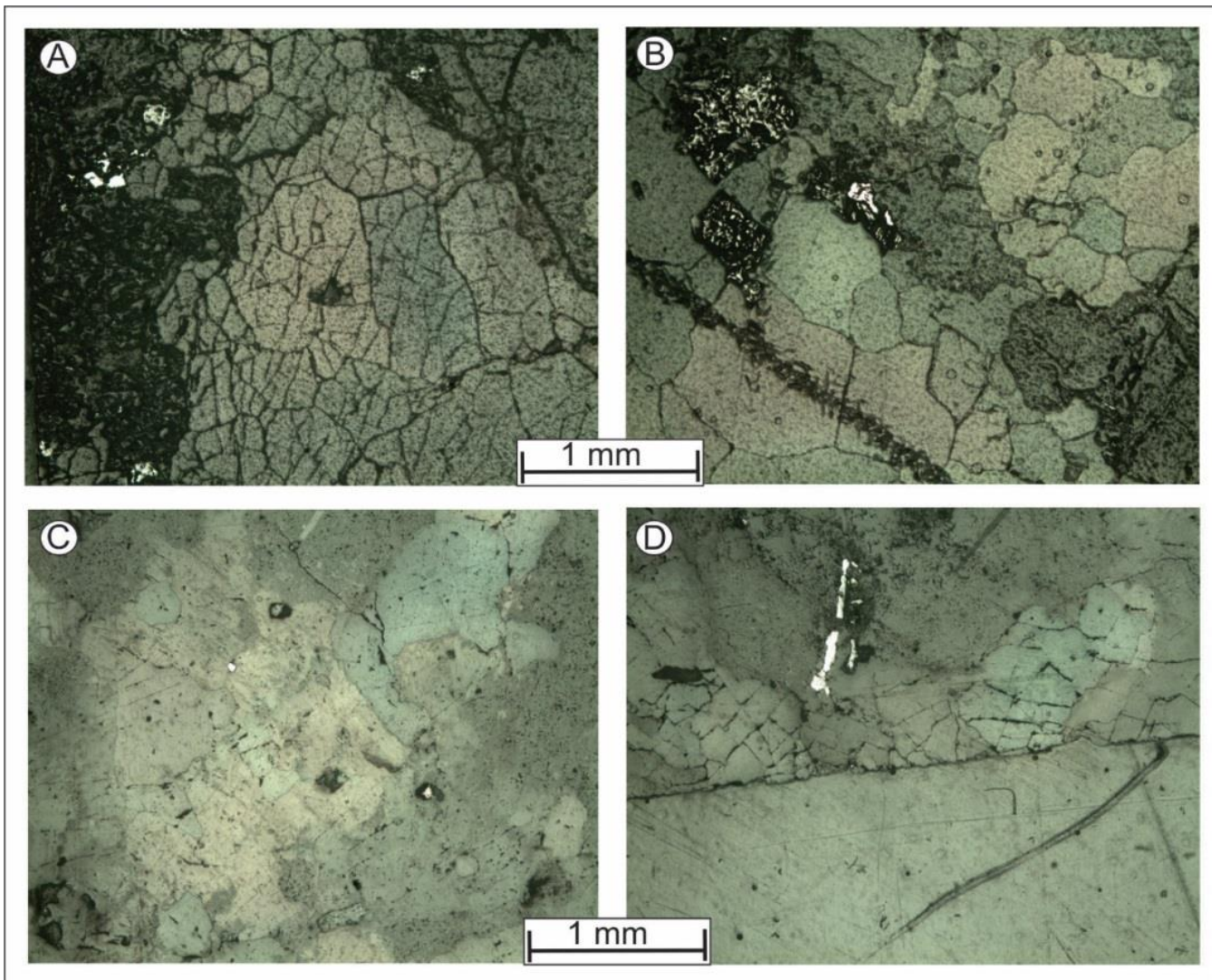

Figura 13 - (A - B) Os cristais cúbicos e sub-angulares de pirita, cor amarelo claro, intensamente alterada; $(C)$ pequenos cristais de pirita cúbica disseminadas na rocha; (D) cristal sub-édricos de ilmenita, cor rosa pálido a amarelado. 


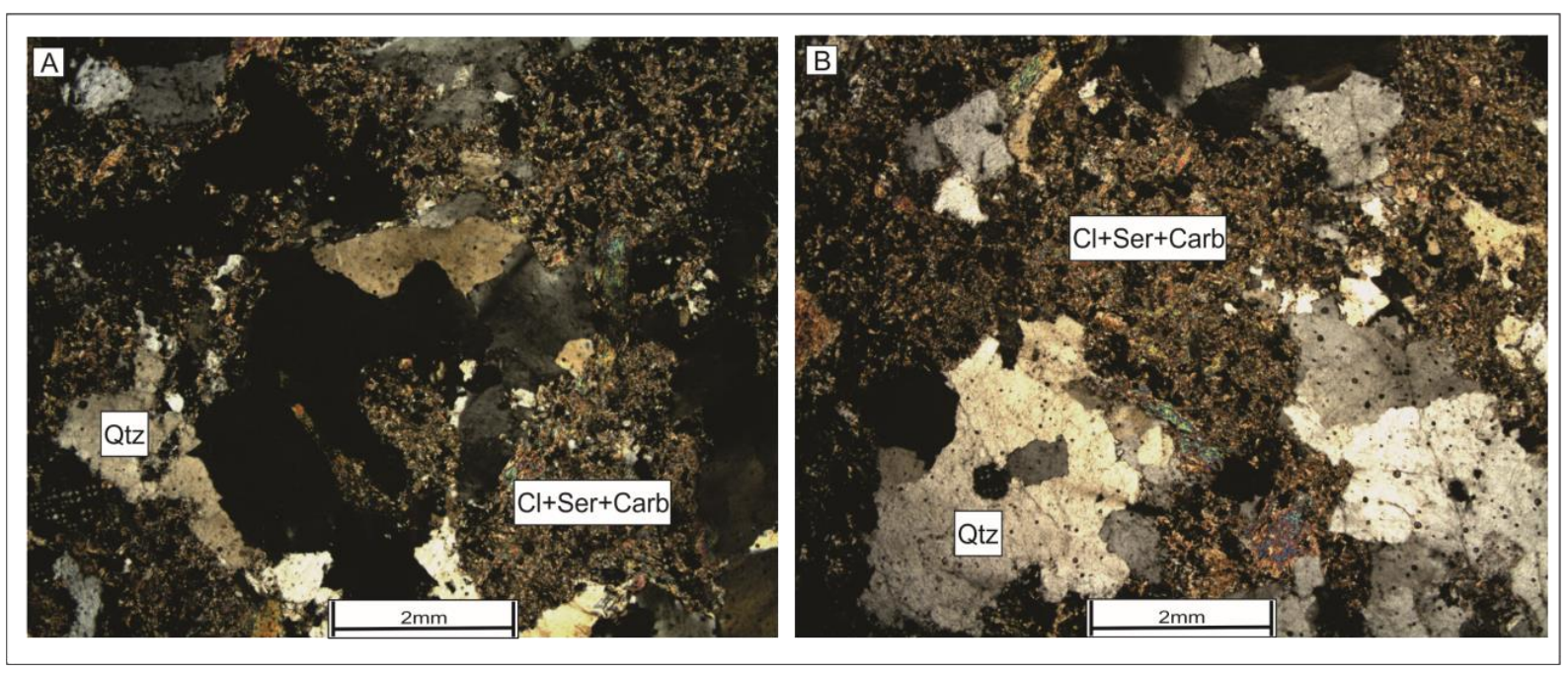

Figura 14 - (A-B) Alteração secundária pervasiva em minerais de plagioclásio, caracterizada por clorita + sericita + carbonato + epidoto \pm pirita. (nicóis cruzados). 


\section{REFERENCIAS DA TESE}

Abreu M.C. 2004. Alteração hidrotermal e mineralização aurífera do depósito de Novo Mundo, região de Teles Pires - Peixoto de Azevedo, Província de Alta Floresta (MT). Trabalho de Conclusão de Curso, IG/UNICAMP, 29p.

Almeida, F.F.M. 1978. A evolução dos crátons Amazônico e do São Francisco comparada com seus homólogos do hemisfério norte. In: CONOR. BRAS. GEOL., 30. Recife, 1978. Anais. Recife, SBO. v. 6, p 2393-2407.

Almeida F.F.M.; Hasui Y.; Brito Neves B.B.; Fuck R.A.; 1981. Brazilian structural provinces: na introduction. Earth Sci. Rev. 17, 1-29.

Alves, C. L.; Sabóia, A. M.; Martins, E. G.; Stropper, J. L.; 2010. Folhas São José do Xingu e Rio Comandante Fontoura. Escala 1:250.000. Goiânia: CPRM (Convênio CPRM/SICME-MT).

Assis, R. R. 2011. Depósitos auríferos associados ao magmatismo granítico do setor leste da Província de Alta Floresta (MT), Craton Amazônico: tipologia das mineralizações, modelos genéticos e implicações prospectivas. Instituto de Geociências, Universidade Estadual de Campinas; Dissertação de Mestrado; 456p.

Barros, M.A.S.B.; Chemale Jr, F.; Nardi, L. V. S.; Lima, E.F.; 2009. Paleoproterozoic bimodal post-collisional magmatism in the southwestern Amazonian Craton, Mato Grosso, Brazil: Geochemistry and isotopic evidence. J. of South American Earth Sciences.27:11-23.

Barros, M. A. S.; Dezula, S. E. M.; Pierosan, R.; Prado, E. S.; Silva, F. R.; 2013. Idade U-Pb SHRIMP do Granito Terra Nova, Suite Intrusiva Teles Pires - Provincia Aurifera de Alta Floresta, Cráton Amazonas. In: $13^{\circ}$ Simpósio de Geologia da Amazônia. CDROM.

Barros, M.A.S.; Barros, A.J.P.; Santos J.O.S.; Rocha, M.L.B.P.; 2015. Extension of The Tapajós Domains to The Alta Floresta Gold Province: Evidence From U-Pb SHRIMP Ages of the Nhandu Intrusive Suíte At 1962 And 1967 Ma. 14 Simpósio de Geologia da Amazônia.

Barros, M.A.S.; Silva, C. H.; Santos J.O.S.; Kenzo, B.; 2016. Reviewing the bacaeri Complex in the southern Amazon Craton using U-Pb geochronology in zircon and monazite implications for the timing of mafic and granitic magmatism during the Colíder Volcanism, In: $10^{\circ}$ SSAGI, PuertoVallarta, Mexico.

Bini, E. G.; Barros, M. A. S.; Pierosan, R.; Santos, J. O. S.; 2015. Petrography and geochronology of felsic volcanic rocks at the eastern portion of Serra do Cachimbo, south-central Amazonian Craton, Brazil. In: VI Simpósio de Vulcanismo e Ambientes Associados, 2015, São Paulo. Boletim de Resumos. v. 1, p. 67.

Bizotto A.C.; 2004. Mineralizações auríferas na região de Teles Pires - Peixoto de Azevedo (Mato Grosso), Província de Alta Floresta: O Depósito de Santa Helena. Trabalho de Conclusão de Curso, Instituto de Geociências, Universidade Estadual de Campinas, Campinas, 38p.

Cordani, U.G.; Teixeira , W.; D'Agrella-Filho, M.S.;Trindade R.I.; 2009. The position of the Amazonian Craton in supercontinents Gondwana Research 15 p396-407.

Cherniak, D.J.; Watson, E.B.; 2001. Pb diffusion in zircon. Chemical Geology 172, 5-24. 
Chu, N.C.; Taylor, R.N.; Chavagnac, V.; Nesbitt, R.W.; Boella, M.; Milton, J. A.; 2002. Hf isotope ratio analysis using multi-collector inductively coupled plasma mass spectrometry: an evaluation of isobaric interference corrections. J. Anal. At. Spectrom., v. 17 , p. $1567-1574$.

Dardene. M.A; Schobbenhaus, C.; 2001. Metalogênese do Brasil. Brasília, Ed Universidade de Brasília, 392p.

DePaolo, D.J.; 1981. A neodymium and strontium isotopic studyof the Mesozoic calcalkaline granitic batholiths of the Sierra Nevada and Peninsular Ranges, California. J. Geophys. Res. 86, 10470-10488.

Dezula, S. E. M.; 2016. Geoquímica e Geocronologia do Granito Aragão - Província Aurífera de Alta Floresta - Craton Amazonas - MT. Dissertação de Mestrado. Universidade Federal de Mato Grosso, 46p.

Goia, S.M.C.L. \& Pimentel, M. M. 2000. The Sm-Nd Isotopic Method in the Geochronology Laboratory of the University of Brasília. An. Acad. Bras. De Cienc., 72(2), 219-245.

Kerr, P.F. 1959. Optical Mineralogy. Mcgraw-Hill. New York, 3ed. 442p.

Klein, E. L., and Koppe, J. C., 2000. Chlorite geothermometry and physicochemical conditions of gold mineralization in the Paleoproterozoic Caxias deposit, São Luís Craton, northern Brazil: Geochimica Brasiliensis, v. 14, p. 219-232.

Lacerda Filho, J.V.; Souza, J.O.; Oliveira, C.C.; Ribeiro, P.S.E.; Boas P.F.V.; Albuquerque, M.C.; Frasca, A.A.S.; Borges, F.R.; Moreton, L.C.; Martins, E.G.; Camargo, M.A; Valente, C.R.; Pimentel, M.M.; Botelho, N.F. 2001, Geologia e evolução tectônica da região norte do Mato Grosso (Projeto Promin-Alta Floresta). In: Simpósio de Geologia da Amazônia, 7, Bélem. Sessão Temática. Belém: SBG.

Lacerda Filho, J.V.; Abreu Filho, W.; Valente, C.R., Oliveira C.C.; Albuquerque, M.C.; 2004. Geologia e Recursos Minerais do Estado de Mato Grosso. Escala 1:1.000.000. Goiânia. CPRM. CD-ROM.

Miguel Júnior, M.; 2011. Mineralizações auríferas do lineamento Peru-Trairão Província Auríifera de Alta Floresta-MT: controle estrutural e idade U-Pb das rochas hospedeiras. Dissertação de Mestrado, Instituto de Geociências, Universidade Estadual de Campinas.

Morel, M.L.A.; Nebel, O.; Y.J. Nebel-Jacobsen, Y.J.; Miller, J.S.; Vroon, P.Z.; 2008. Hafnium isotope characterization of the GJ-1 zircon reference material by solution and laser-ablation MC-ICPMS. Chemical Geology 255, 231-235.

Moreton, L.C. \& Martins, E.G. 2003. Geologia e Recursos Minerais da Folha Vila Guarita Folha SC.21-Z-B. Estado de Mato Grosso. Escala 1:250.000. Brasília: CPRM.

Moreton, L.C. \& Martins, E.G. 2005. Geologia e Recursos Minerais de Alta Floresta. Vila Guarita. Escala 1:250.000. Brasília, Serviço Geológico do Brasil, CPRM, 68 p.

Moura, M.A. 1998. O maçiço granítico Matupá no depósito de ouro Serrinha (MT): petrologia, alteração hidrometal e metalogenia. Tese de Doutorado, Instituto de Geociências, Universidade de Brasília. 238 p.

Moura, M.A.; Botelho N.F.; Olívio G.R.; Kyser T.K.; 2006. Granite-related Paleoproterozoic, Serrinha gold deposit, Southern Amazonia, Brazil: hydrothermal alteration, fluid inclusion and stable isotope constraints on genesis and evolution. Econ. Geol., 101:585-605. 
Paes de Barros, A.J.; 1994. Contribuição a geologia e controle das mineralizações auríferas de Peixoto de Azevedo - MT. Instituto de Geociências, Universidade de São Paulo. São Paulo-SP. Dissertação (Mestrado em Geologia Econômica e Hidrogeologia), 145 p.

Paes Barros, A.J.P.; 2007. Granitos da região de Peixoto de Azevedo - Novo Mundo e mineralizações auríferas relacionadas - Província Aurífera Alta Floresta (MT). Instituto de Geociências, Universidade Estadual de Campinas, Tese de Doutorado, 154p.

Patchett, P.J.; Kuovo, O.; Hedge, C.E.; Tatsumoto, M.; 1981. Evolution of continental crust and mantle heterogeneity: evidence from Hf isotopes. Contrib. Mineral. Petrol. 78, 279297.

Pimentel, M.M. \& Botelho, N.F. 2001. Geologia e Evolução Tectônica da Região Norte do Mato Grosso (Projeto Promin-Alta Floresta). In: Simpósio de Geologia da Amazônia, 7, Belém. Resumos Expandidos... Belém: SBG - Núcleo Norte. Cd-Rom.

Pinho, M.A.S.; Chemale Jr. F.; Schmus W.R.V.; Pinho F.E.C. 2003. U-Pb and Sm-Nd evidence for 1.76-1.77 Ga magmatism in the Moriru region, Mato Grosso, Brazil: implications for province boundaries in the SW Amazon Cráton. Prec. Res., 126: 1-25.

Prado, E.S.; Barros M.A.S.; Pinho F.E.C.; Pierosan R.; 2013. Granito Terra Nova petrologia e geocronologia: um granito tipo-A da Província Aurífera Alta Floresta Cráton Amazônico. Rev. Bras. Geoc. 43(1): 101-116.

Sato, K., Basei M.A.S. \& Siga O.J. 2008. Novas técnicas aplicadas as método U-Pb no CPGeo - IGc/USP: avanços na digestão química, espectrometria de massa $\left(\mathrm{TIM}_{\mathrm{S}}\right)$ e exemplos da aplicação integrada com SHRIMP. Geologia USP - série Científica, 8: 7799.

Santos, J.O.S. 2000. Os terrenos Paleoproterozóicos da Província do Tapajós e as mineralizações de ouro associadas. Tese de Doutorado, Universidade Federal do Rio Grande do Sul, Porto Alegre, v.1, 208p.

Santos, J.O.S.; Groves, D.I.; Hartmann, A.; Moura, M.A.; McNaughton, N.J.; 2001. Gold deposits of the Tapajós and Alta Floresta domains, Tapajós-Parima orogenic belt, Amazon Craton, Brazil. Mineralium Deposita, 36:278-299.

Santos J.O.S. 2003. Geotectônica dos escudos das Guianas e Brasil- Central. In: Bizzi L.A., Schobbenhaus C., Vidotti R.M., Gonçalves J.H. (eds). Geologia, tectônica e recursos minerais do Brasil. CPRM. Brasília. CD-ROM.

Santos J.O.S. 2006. A compartimentação do Cráton Amazonas em províncias: avanços ocorridos no período 2000-2006. In: SBG, Simpósio de Geologia da Amazônia, 9, 1 CD-Rom.

Santos, J. O. S.; Rizzotto, G.J.; Potter, P.E.; McNaughton, N.J.; Matos, R.S.; Hartmann, L.A.; Chemale Jr.F.; Quadros, M.E.S.; 2008. Age and autochthonous evolution of the Sunsás Orogen in West Amazon Craton based on mapping and U Pb geochronology. Precambrian Research, v. 165, p. 120-152.

Santos, J. O. S.; Barros, M. A.; Barros, A. J. P.; McNaughton N.; 2015. Datação de Titanita usando a relação $\mathrm{Pb}^{208} / \mathrm{Th}^{232}$ : caso do Gnaisse Gavião, Província Aurífera Alta Floresta In: $14^{\circ}$ Simpósio de Geologia da Amazônia.

Scherer, E.; Münker, C.; Mezger, K.; 2001. Calibration of the lutetium-hafnium clock. Science 293, $683-687$. 
Silva, G.H.; 1974. Esboço Geológico de parte da Folha Sc.21. Juruena. In: Congresso Brasileiro de Geologia, 28, 1974, Porto Alegre. Anais... Porto Alegre: SBG,, V.4, P.309-320.

Souza, A.M.M.; Faria, C.A.S.; Landin, J.P.P.; Leal, J.W.L.; 1979. Reconhecimento geológico no limite Mato Grosso - Pará, Projeto São Manuel. Brasília, DNPM/CPRM, 27p.Il.

Souza, J.O.; Frasca, A.A.S.; Oliveira, C.C.; 2004. Projeto Província Mineral de Alta Floresta. Geologia e Recursos Minerais das folhas Rio São João da Barra (SC.21-V-D), Alta Floresta (SC.21-X-C), Ilha 24 de Maio (SC.21-Z-A); Vila Guarita (SC.21-ZB), estados de Mato Grosso e Pará. Escala 1:250.000. Brasília: CPRM.

Souza J.P.; Frasca A.A.S.; Oliveira C.C.; 2005. Geologia e Recursos Minerais da Província Mineral de Alta Floresta. Relatório Integrado. Brasília, Serviço Geológico Brasileiro, CPRM,164 p.

Silva M.G. \& Abram M.B. 2008. Projeto Metalogenia da Província Aurífera JuruenaTelesPires, Mato Grosso. Goiânia, Serviço Geológico Brasileiro, CPRM, 212 p.

Stern, R. A. 2001. A new isotopic and trace element standard for the Ion Microprobe: Preliminary thermal ionization massspectrometry $\left(\mathrm{TIM}_{\mathrm{S}}\right) \mathrm{U}-\mathrm{Pb}$ and electronic microprobe data. Report 14, Geological Survey of Canadá, Current Research 2001-F1, $11 \mathrm{p}$.

Tassinari, C.C.G. \& Macambira, M.J.B. 1999. Geological provinces of the Amazonian Craton. Episodes, v. 22, p. 173-182.

Tassinari C.C.G.; Bettencourt J.S.; Geraldes M.C.; Macambira M.J.B.; Lafon J.M.; 2000. The Amazonian Craton. In: Cordani U.G., Milani E.J., Thomas Filho A., Campos D.A. Tectonic Evolution of South America. Rio de Janeiro, SBG, p. 41-95.

Teixeira, W.; Tassinari, C.C.G.; Cordani, U.G.; Kawashita, K. 1989. A Review Of The Geochronology Of The Amazonian Craton: Tectonic Implications. Precambrian Res., 42: $213-227$.

Teixeira, W.; Ávila, C.A.; Dussin, I.A.; Corrêa Neto, A.V.; Bongiolo, E.M.; Santos, J.O.; Barbosa, N.S.; 2015. A juvenile accretion episode (2.35-2.32Ga) in the Mineiro belt and its role to the Minas accretionary orogeny: Zircon U-Pb-Hf and geochemical evidences. Precambrian Research, v. 256, p. 148-169.

Thirwall, M.F.; Walder, A.J.; 1995. In situ hafnium isotope ratio analyses of zircon by inductively coupled plasma mass spectrometry. Chemical Geology 122, 241-247.

Trevisan, V. G.; Xavier, R. P.; Rodrigues, R. M.; Assis, R.R.; Paes de Barros, A. J.; 2012. Regime de fluidos em depósitos de ouro associados a intrusões paleoproterozóicas da Província Aurífera de Alta Floresta (MT), Cráton Amazônico. In: XX Congresso Interno de Iniciação Científica da Unicamp, Campinas - SP.

Trevisan, V. G.; 2015. Estudo comparativo entre mineralizações filonares de $\mathrm{Au}+\mathrm{Cu}$ e $\mathrm{Au}$ + metais base do setor leste da Província Aurífera de Alta Floresta (MT) Cráton Amazônico. Instituto de Geociências, Universidade Estadual de Campinas, Dissertação de Mestrado.

Vasquez, M. L.; Rosa-Costa, L. T.;(Org.) 2008. Geologia e recursos minerais do Estado do Pará: texto explicativo do mapa geológico e de recursos minerais do Estado do Pará. Belém: CPRM,. 328 p. il. color. escala 1:1.000.000. Programa Geologia do Brasil (PGB). 
Woodhead, J.D.; Hergt, J.M.; 2005. A preliminary appraisal of seven natural zircon reference materials for in situ hf isotope determination. Geostandards and Geoanalytical Research 29, 183-195. 


\section{CAPÍTULO - IV I ARTIGO}




\section{ARTIGO I:}

“ ISOTOPE GEOLOGY OF THE GRANITIC ROCKS FROM TRAIRÃO DEPOSIT ALTA FLORESTA GOLD PROVINCE - SOUTHERN AMAZONIAN CRATON"

\section{Authors:}

Mara Luiza Barros Pita Rocha ${ }^{1}$

Farid Chemale Junior ${ }^{1}$

João Orestes Schneider Santos ${ }^{2}$

Marcia Aparecida de Sant'Ana Barros ${ }^{3}$

Francisco Egídio Cavalcante Pinho ${ }^{3}$

Paulo Cesar Correa da Costa ${ }^{3}$

1- Universidade de Brasília - UnB, Brasília, Brazil

2- University of Western of Australia - UWA, Perth, Australia

3- Universidade Federal do Mato Grosso - UFMT, Cuiabá, Brazil

Artigo submetido à revista "Journal of South American Earth Sciences". 


\title{
“ ISOTOPE GEOLOGY OF THE GRANITIC ROCKS FROM TRAIRÃO REGION - ALTA FLORESTA GOLD PROVINCE - SOUTHERN AMAZONIAN CRATON"
}

\author{
Authors: \\ Mara Luiza Barros Pita Rocha ${ }^{1}$ - Farid Chemale Junior ${ }^{1-}$ João Orestes Schneider Santos ${ }^{2}-$ \\ Marcia Aparecida de Sant'Ana Barros ${ }^{3}$ - Francisco Egídio Cavalcante Pinho ${ }^{3}$ - Paulo César \\ Corrêa da Costa $^{3}$ \\ 1-Universidade de Brasília - UnB, Brasília, Brazil \\ 2-University of Western of Australia - UWA, Perth, Australia \\ 3-Universidade Federal do Mato Grosso - UFMT, Cuiabá, Brazil
}

\begin{abstract}
Two distinct groups of biotite granites occur in the Trairão deposit, eastern of Alta Floresta Gold Province, Amazonian Craton. The first one, represented by biotite syenogranite, have calc-alkaline geochemical affinity with high potassium series generated in a mature continental magmatic arc. These rocks have U-Pb SHRIMP crystallization ages between $1969 \pm 2$ to $1923 \pm 17$ Ma with 2030 Ma inherited zircon, attributed to the Cuiú-Cuiú Complex. Metaluminous, K-rich calc-alkaline monzogranites are intrusive in the biotite syenogranites between $1878 \pm 7$ to $1837 \pm 13$ Ma. Based on Th, U, Y, Yb and Hf content, the dated zircon grains have typical continental crust signature. Indeed, Lu-Hf isotopic studies carried out in the same zircon crystals show the following variations: 1) $1969-1923 \mathrm{Ma}$ Granites (initial epsilon Hf values range from -4.1 to -); 2) 1878 - 1837 Ma granites (initial epsilon Hf values range from -3.1 to -9.5$)$. WR $\mathrm{Nd}$ data display eNd values from +0.05 to 6.7 and $\mathrm{Nd}$ model ages of 2.25 to $2.54 \mathrm{Ga}$. The first granites group is correlated with the granites of the Nhandu Intrusive Suite while the youngest granites are correlated with the Matupá Intrusive Suite. Our studies suggest that these granites formed in a continental magmatic arc tectonic setting with two main magmatic pulses between 1960 to 1840 Ma with melting of granitic magma with contribution of crustal and juvenile material in different proportions.
\end{abstract}

Keywords: Amazonian Craton, Granites, U-Pb / SHRIMP, Lu-Hf, Alta Floresta Gold Province

Tese de Doutorado - Mara Luiza Barros Pita Rocha 


\section{1-INTRODUCTION}

The Alta Floresta Gold Province (AFGP) occurs between Ventuari - Tapajós (1.95 to $1.8 \mathrm{Ga}$ ) and Rio Negro - Juruena (1.8 to $1.55 \mathrm{Ga})$ geochronological provinces, according to Tassinari \& Macambira (1999) or Tapajós - Parima (2.03 to 1.88 Ga) and Rondônia - Juruena (1.82 to $1.54 \mathrm{Ga}$ ) provinces according to Santos et al. (2000) model. Regardless of the model adopted, the provinces are composed of plutono - volcanic units generated in magmatic arc environment, which developed and progressively added to the protocraton formed by the Central Amazonian Province during the Paleoproterozoic Tassinari \& Macambira 1999; Souza et al., 2005 ; Santos et al. 2006; Silva \& Abram 2008).

The main of gold, copper-gold and base metals deposits occurring in the Amazonian Craton are situated in the Carajas Gold Province (Archean) and the Tapajos and Alta Floresta Gold Provincies (Proterozoic) (Bettencourt, et al. 2016). The magmatic history of granites and associated mineralization, has been a frequent subject of several investigations. Different hypotheses have been proposed for gold deposits related to granites: lode type (Paes de Barros, 1994; Santos et al., 2001; Teixeira, 2015); epizonal deposits - intrusion-related gold systems (Santos et al., 2001; Paes de Barros, 2007; Abreu, 2004; Bizotto. 2004); porphyry gold (Moura 1998; Moura et al., 2006; Assis, 2011; Trevisan, 2012; Trevisan, 2015) and epithermal gold mineralization (Assis, 2011; Trevisan, 2015). Petrological and isotopic characterization of host rocks is an important key for the understanding of the Amazonian Craton tectonic evolution is importance for the understanding of the metallogenic processes.

The present work emphasizes the petrographic, geochemical and geochronological characterization of granitic bodies associated with the Trairão gold occurrence, Novo Mundo district, eastern Alta Floresta. LA-MC-ICPMS and SHRIMP U/Pb zircon ages from six samples and $\mathrm{Sm}-\mathrm{Nd}$ isotopic data from four samples are presented to discuss the magma source and tectonic environment of the granites at the Trairão region, where occur the Trairão and Chumbo Grosso gold deposits.

\section{2- GEOTECTONIC CONTEXT OF THE AMAZONIAN CRATON}

The Amazonian Craton was formerly considered by Amaral (1974) and Almeida et al. (1981) as a large Archean platform, partially reworked and reactivated during the Proterozoic, resulting in intense anorogenic felsic magmatism. During the 1970 decade new 
ideas about plate tectonics accopled with intense production of isotopic ages in the Amazonian Craton, allows to researchs a new understanding about the evolution of this craton. Cordani (1979) and Santos (2000) present an evolution model based in accrestionary arcs evolution. According with these authours, there is in the Amazonian Craton an Archean nucleous surrounded by youngest mobile arcs, from west to east accrestion.

The continuous acquisition of geochronological data $(\mathrm{Rb} / \mathrm{Sr}$ and $\mathrm{U} / \mathrm{Pb})$ designates that the Amazonian Craton grew through continued Proterozoic soft-collision/accretionary processes driven by northeasterly B-type subductions, surrounding an Archean core (Teixeira et al., 1989; Tassinari and Macambira, 2004; Cordani and Teixeira, 2007, Bettencourt, et al., 2016). These works resulted in the subdivision of Amazonian Craton into two Archean nuclei and five Proterozoic geotectonic provinces. The same tectonic structure was reinforced, with modifications of some provinces by Santos et al. (2003) and Santos (2008), based on U/Pb SHRIMP zircon and $\mathrm{TIM}_{\mathrm{S}}$ data from a few hundred samples, as well as $\mathrm{Pb} / \mathrm{Pb}$ evaporation. (Bettencourt, et. al. 2016), Fernandes et al. (2011) and Juliani et al. (2013, 2014) based on field work, geochemistry, geophysical studies and isotope geology indicate that the southern part of Amazonian Craton is essentially composed of two continental magmatic arcs that evolved between ca. 2.0 and $1.86 \mathrm{Ga}$, named Tapajos Arcs, with a possible flat subduction stage, under an Archean Paleoproterozoic continent. This propose contrast with the original model from Cordani (1979) and Santos et al. (2000) that considered the Proterozoic provinces as an island arc.

Santos (2003) defined that the Rondônia-Juruena Province consists of three domains: Jamari, Juruena and Alto Jauru. Lacerda Filho et al. (2004) redefined the Rio Negro-Juruena Province as $(1.85$ to $1.75 \mathrm{Ga}$ ) and recognized the existence of three domains (excluding Jamari Domain): Juruena (1.85 to $1.75 \mathrm{Ga}$ ), Roosevelt- Aripuana (1.76 to $1.74 \mathrm{Ga}$ ) and Jauru (1.79 to $1.72 \mathrm{Ga}$ ). Santos (2003) propose five magmatic arcs in the Rio Negro-Juruena Province: Quatro Cachoeiras collisional arc (1670-1630 Ma); Roosevelt continental arc (1740 Ma); Jamari islands arc and Jauru continental arc (1740-1760 Ma); including Colíder, Monte Verde, Vitoria, São Romão magmatic arcs (1770 - 1785 Ma); Cristalino, Paranaíta , Juruena and Moriru magmatic arcs (1800 - $1820 \mathrm{Ma}$ ); and Bacaeri island arc (older than 2.0Ga).

According to Lacerda Filho et al. (2004) the Juruena Domain consists of an Andeantype felsic plutonic and volcanic rocks, calcium alkaline nature with high potassium, formed between 1820 and $1760 \mathrm{Ma}$. In the Juruena magmatic arc (1.85 to $1.75 \mathrm{Ga}$ ), Lacerda Filho et 
al. (2004) and Souza et al. (2005) described two terrains, an accretionary, deformed in the ductile regime, medium to high metamorphic grade and another plutonic volcanic, postcollisional, sin to tardi-orogenic. According to these authors, the accretionary terrain is represented by units called Nova Monte Verde and Bacaeri-Mogno Complexes. However, Santos (2003) suggests a breakdown of the Complex, by recognizes charnockites with features of post-tectonic rocks on the Mogno Complex, and tonalites, diorite and basalt, with juvenile arc characteristics and associated to the Bacaeri portion, turbidites metamorphosed to amphibolite facies.

Santos et al. (2003) divide this portion of the Amazonian craton in two metallogenic domains: Alta Floresta and Tapajos. In this work we adopt the Santos et al. (2008) terminology (Figure 1).

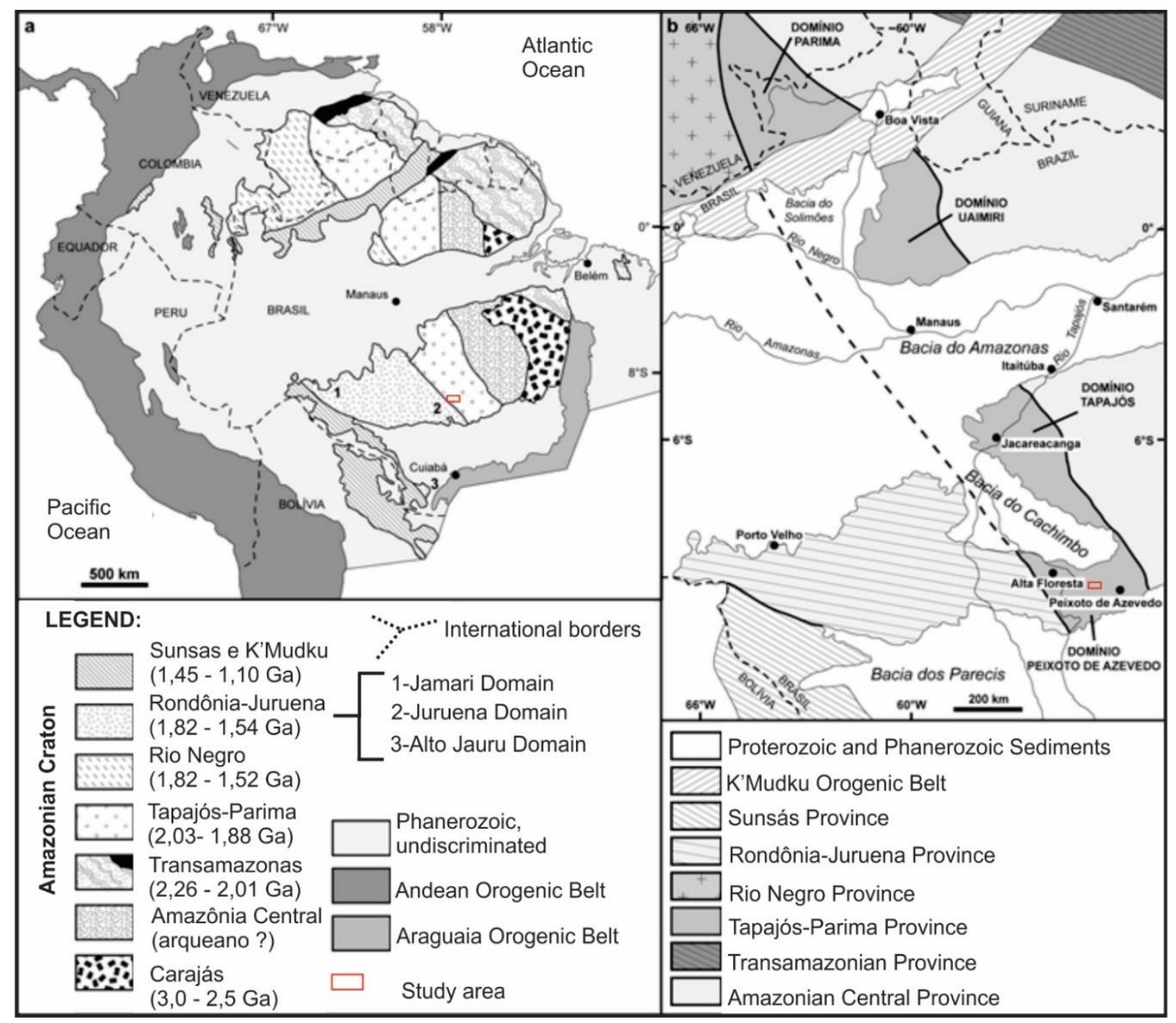

Tese de Doutorado - Mara Luiza Barros Pita Rocha 
IV Figure 01 - Amazonian Craton geotectonic maps. (A) Geotectonic model by Santos et al. (2003); (B) Different metallogenic sub-domains of the Tapajós-Parima Province, Santos et al. (2008).

\section{1- Alta Floresta Region Geotectonic Setting}

The Alta Floresta - Peixoto de Azevedo region stood out during the years 1970-1998 as one of the largest gold-producing regions of Brazil. Due to this fact several metallogenic terminology have been proposed for the region.

The Alta Floresta Gold Province (AFGP) is located in the south central portion of the Amazonian Craton (Almeida, 1978 and Almeida et al. 1981). According to the Santos et al. (2000; 2006; 2008) model the AFGP is between the tectonic provinces Tapajós - Parima (2.1 to $1.87 \mathrm{Ga}$ ) and Rondônia - Juruena $(1.82$ to $1.54 \mathrm{Ga}$ ), or by the model of Tassinari \& Macambira (1999) the AFGP is between Ventuari - Tapajós (1.95-1.80 Ga) and Rio Negro Juruena (1.8 - $1.55 \mathrm{Ga})$ provincies.

According to Silva \& Abram (2008) the tectonic evolution of the Alta Floresta Gold Province can be divided in seven phases: (I) generation of rocks set in a tectonic extensional environment (Mogno portion from the Bacaeri-Mogno complex); (II) generation of the CuiúCuiú and Juruena magmatic arcs with calcium alkaline affinity from an active continental margin; (III) collisional phase represented by ths Apiacas Granites (1784 $\pm 32 \mathrm{Ma})$ and older Apiacas Granites (1871 $\pm 21 \mathrm{Ma})$; (IV) crustal delamination and consequent generation of intraplate magmatism (Flor da Serra); (V) basin generation (intra-arc), and volcano sedimentary sequence into this basin (São Marcelo Cabeço Group); (VI) closing of the orogen, accompanied by progressive deformation, with the mega generation transcurrent shear zones and (VII) generation of the units Nova Cannaã Suite and Teles Pires Granites as a later phase.

The study area is located in the eastward portion of the Alta Floresta Gold Province. Most studies that have been carried out (Assis, 2011; Paes de Barros, 2007 and Miguel Jr., 2011). A great contribution to the stratigraphic knowledge of the region was given by Brazil's Geological Survey at the beginning of this century. Due to the regional scale mapping and difficulties to access the region, there is still much controversy in the stratigraphic position of the rocks. 
In this work is presented a proposal for a geological map based on the mapping of the Brazil's Geological Survey and database available in the literature containing geochronological results over the last fifteen years. The Figure 2 displays the geological subdivision proposed in this work to the eastward portion of the Alta Floresta Gold Province.

\section{3-GEOLOGICAL BACKGROUND}

The Alta Floresta Gold Province can be even described the moment as constituted by units: Archean Basement; Cuiu-Cuiu complex; Nhandu Intrusive Suite; Matupá Intrusive Suite, Juruena Intrusive Suite, Colíder Group, Teles Pires Volcano - Plutonic Suite and sedimentary covers.

\section{1-The Archean basement}

Described by Paes de Barros (2007) for the Gavião gneiss, which occurs near the Peixoto de Azevedo city. However, the Archaean dating refer to a single outcrop, thus becoming very restricted dimensions to be evidence of a heterogeneous basement with the presence of an Archean crust. Santos et al. (2015) presented zircon and titanite U/Pb data for this outcrop. The obtained data for zircons show two ages $2.8 \mathrm{Ga}$ and $2.6 \mathrm{Ga}$ while titanite show 1870 Ma that can represent metamorphic ages of the Archean basement.

The Cuiú-Cuiú complex was described in the Tapajós Domain and extended for Alta Floresta region by Souza et al. (2005). It consists of orthogneisses of monzonitic, tonalite and granite composition. They occur in the form of elongated narrow strips discontinuous and not always mappable. Klein et al. (2000) describes this unit as typical of root of volcanic arc environment. These orthogneisses have been data by U-Pb SHRIMP dating at $2005 \pm 7 \mathrm{Ma}$ (Santos, 2000) and at $2008 \pm 3 \mathrm{Ma}$ (Dezula, 2016). The latter rocks occur south of the Novo Mundo, near the Aragão deposit.

\section{2-Nhandu Intrusive Suite}

The term Nhandu was introduced by Souza et al. (1979) to refer to the porphiroblastic granitic of granodioritic to tonalitic composition, they present massive structure and pseudo rapakivi texture. According to Lacerda Filho (2001 and 2004) they show an alkaline calcium affinity with high potassium, I type, represented by a red color magnetite-biotite granite. 
Initially called Nhandu Granite, the term was later elevated to the category of suite by Moreton \& Martins (2003 and 2005). Zircon U/Pb (SHRIMP) dating, set a time interval between $1967 \pm 7$ and $1962 \pm 6$ Ma for Nhandu Intrusive Suite, correlating it with the Creporizão Intrusive Suite, thus, extending the Tapajós metallogenic domain to the northern portion of the Alta Floresta Gold Province (Barros et al. 2015).

\section{3-Matupá Suite}

The Matupa Intrusive Suite term was originally adopted by Moura et al. (1998) to particularize a body of isotropic biotite monzogranite which is located to the south of the Matupá city. It comprises an oxidized magmatism, calcium alkaline with high potassium, magnesium and peraluminous to slightly metaluminous. The Matupá Granite (Paes de Barros 1994, 2007) occur NW-SE elongated bodies at the Serrinha deposit region. The Matupá granite in the Serrinha region (Moura et al. 1998) outcrops as not deformed and little fractured blocks, has gold mineralization in quartz vein and porphyry type (disseminated and stockwork) that are associated with a major hydrothermal stage. A zircon $\mathrm{Pb}-\mathrm{Pb}$ age of 1,872 $\pm 12 \mathrm{Ma}$ was obtained as well as model ages $\left(\mathrm{T}_{\mathrm{DM}}\right)$ that vary in the range 2.47 to $2.34 \mathrm{Ga}$, and $\varepsilon N d(t)$ ranging from -2.7 to -4.3 (Moura et al. 1998). Silva \& Abram (2008) obtained Nd $\mathrm{T}_{\mathrm{DM}}$ model age of $2.15 \mathrm{Ga}$ with $\varepsilon \mathrm{Nd}(\mathrm{t}=1.87 \mathrm{Ga})$ of -0.98 . Recent $\mathrm{U} / \mathrm{Pb}$ (SHRIMP) datings made by Silva et al. (2014), showed that a biotite monzogranite facies previously defined as belonging to the Peixoto Granite, has an age of $1869 \pm 10 \mathrm{Ma}$, similar to the biotite monzogranites from the Matupá Intrusive Suite.

\section{4-Juruena Intrusive Suite}

The name Juruena Intrusive Suite was given by Silva et al (1974) for a set of aligned NW-SE trend granitic rocks, generally they are gneissic and ellipsoidal. Zircon U/Pb age show $1817 \pm 12 \mathrm{Ma}$ (Silva \& Abram, 2008). Geochemically the rocks that make up this suite are calcium alkaline with high potassium, metaluminous to peraluminous, undeformed and no magnetic, REE patterns are LREE enriched with a HREE slightly fractionated, similar to granitic rocks of active continental margin arc (Souza et al. 2004). In this paper, we consider the Paranaíta Intrusive Suite and Apiacás Granite as part of the Juruena Suite based on zircon $\mathrm{U} / \mathrm{Pb}$ age of $1808 \pm 14 \mathrm{Ma}$ obtained by Silva \& Abram (2008), compatible with age Juruena age. 


\section{5-Colíder Group and Intrusive Suites of same age}

This group is represented by volcanic rocks (effusive and pyroclastic) showing intermediate to acid composition, high potassium, calcium alkaline, peraluminous to metaluminous that show geochemical affinity with orogenic granitic series (Moreton \& Martin, 2005). These rocks show tectonic boundaries contacts with the Matupa and Nhandu Intrusive Suite, besides having $\mathrm{U} / \mathrm{Pb}$ zircon ages $1785 \pm 6,3$ and $1781 \pm 8 \mathrm{Ma}$ and $2.34 \mathrm{Ga}$ $\mathrm{T}_{\mathrm{DM}}$ model age and $\varepsilon N d(\mathrm{t})-3,75$ (Pimentel 2001, Silva \& Abram 2008). Granitic rocks of similar have been described as São Pedro Granite (Souza et al. 2005); São Romão Granite and Mogno Intrusive Suite (Barros et al. 2016).

\section{6-Teles Pires Vulcano-Plutonic Suite}

In this work a group of units are included in the Teles Pires Volcano-Plutonic Suite as: acidic volcanic rocks of southern Serra do Cachimbo (Bini, et al. 2015), Granite Terra Nova (Prado et al. 2013), in addition to the units already described as part of the Teles Pires magmatism (Pinho et al. 2003 and Barros et al. 2009). The Teles Pires Intrusive Suite, described by Souza et al. (2005) includes plutonic and subvolcanic rocks, consisting mostly by alkali feldspar granite and syenogranite, monzogranite and granodiorites are subordinated. Zircon $\mathrm{U} / \mathrm{Pb}$ dating set an age of $1757 \pm 16 \mathrm{Ma}$ and a $2.1 \mathrm{Ga} \mathrm{T}_{\mathrm{DM}}$ age for the Teles Pires granite (Santos, 2000). Based on U/Pb isotopic data and $\mathrm{T}_{\mathrm{DM}}$ values, Pinho et al. (2003) included the bimodal volcanism of Moriru region, north of Aripuana, volcanic felsic rocks with $\mathrm{U}-\mathrm{Pb}$ ages (TIMs) between $1770 \pm 6 \mathrm{Ma}$ to $1778 \pm 6 \mathrm{Ma}$ and $\mathrm{Nd} \mathrm{T}_{\mathrm{DM}}$ model ages 2.0 to 2.1 Ga to Teles Pires Magmatism. Bini et al. (2015) dated rhyolites from the southern of the Serra do Cachimbo by U/Pb (SHRIMP) obtained an age of $1757 \pm 14 \mathrm{Ma}$. This last age and considered the closest to the expected for this post-collisional to anorogenic magmatism.

\section{4- Analytical Procedures}

\subsection{Petrography and Lithogeochemistry}

Thin sections of 30 granites samples and polished sections of 20 samples (granites and mineralized veins and breccias) were prepared for qualitative ad quantitative petrographic analysis at the Sample preparation lab of the Institute of Geosciences of Universidade de Brasília (UnB) and Center of Microscope, Characterization and Microanalysis of University 


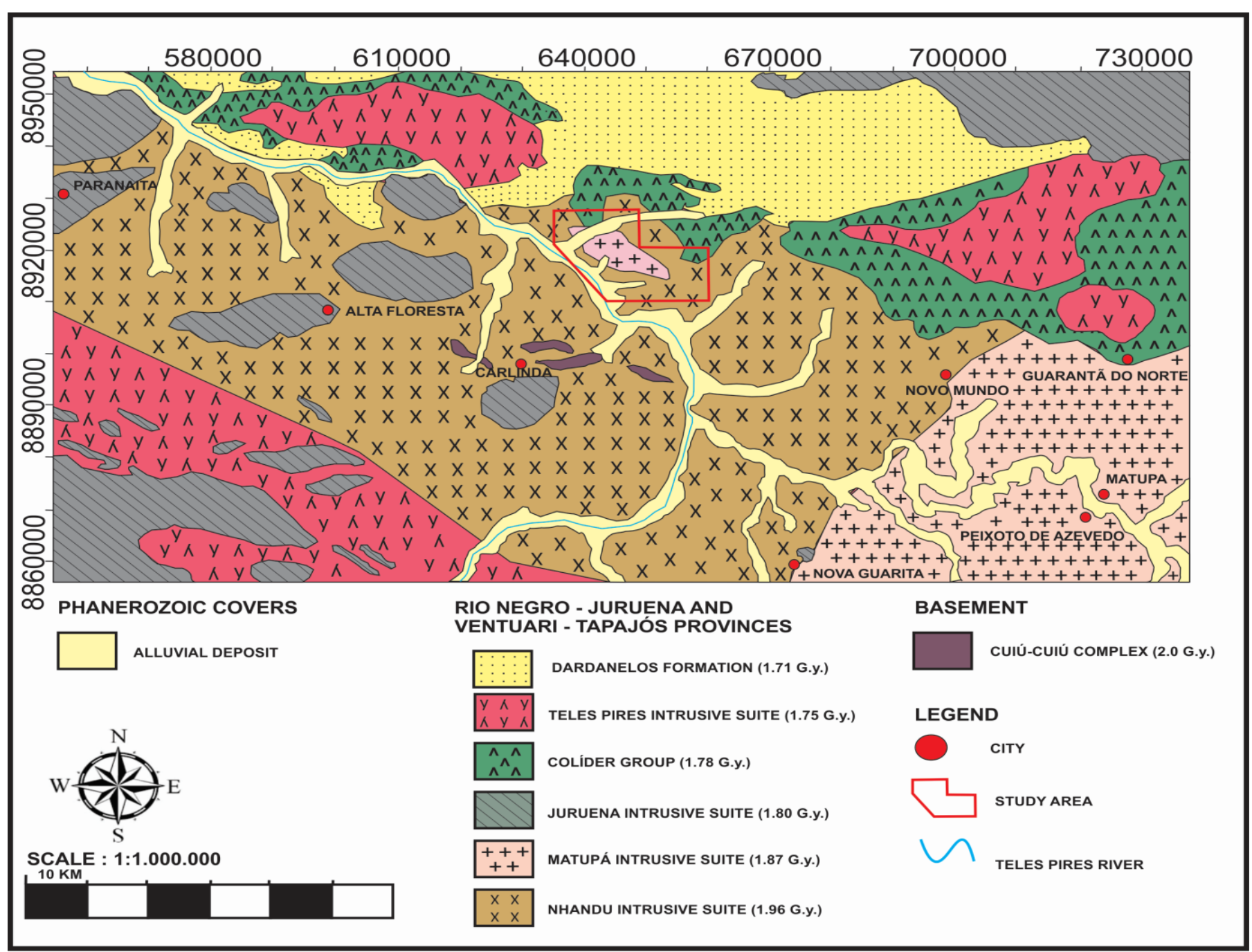

IV Figure 02 - Regional geological map of the eastern part of the AFGP, with location of the study area, modified Miguel Jr. et al. 2011. 
of Western Australia (UWA). The microscopic studies were carried out with Zeiss Imager A2m and Olympus BX60 optical microscopes to characterize the mineral composition (with mineral proportion), textural aspects, and mineral alteration.

Major, trace, and rare-earth (REE) elements were analyzed in 13 granitic samples at the Acme Analytical Laboratory Ltda (Vancouver, Canada). Major oxides were analyzed by ICP-AES (Inductively Coupled Plasma-Emission Spectrometry) and trace and REE were analyzed by ICP-MS (Inductively Coupled Plasma-Mass Spectrometry). The major, trace element and REE chemistry is reported in the table 1.

\subsection{Mineral Chemistry}

Zircons of 6 samples of the Trairão region, which were analyzed by SHRIMP U-Pb and LA-MC-ICPMS Lu-Hf methods, were examined after the textural, shape and major and trace chemistry. Zircon grains were hand picked and mounted in epoxy in 2.5-cm diameter circular grain mounts and polished until the zircons were revealed.

Backscatering eletron (BSE) images and pontual qualitative analyses by EnergyDispersive Spectrometry (EDS) and quantitave analyses by Wavelenght-Dispersive Spectrometry (WDS) were acquire at the Eletron Microprobe Labotory of UnB with a JEOL JXA-8230 Electron Probe Microanalyzer. Major elemens were analyzed with conditons of $15 \mathrm{kV}$ of voltage, $20 \mathrm{nA}$ of beam current and counting time of $10 \mathrm{~s}$ (on the peak, 5s determining background) under 1x10-4 MPa vacuum conditions to oxide element. Hf was analyzed with different conditions: $20.0 \mathrm{kV}, 20 \mathrm{nA}$ and $20 \mathrm{~s}$ to peak, while trace elements (Yb, Ta, Y, U, Th) with conditons of voltage of $20 \mathrm{kV}$, current $100 \mathrm{nA}$ and counting time of 100s. The mineral chemistry of $\mathrm{Zr}, \mathrm{Si}, \mathrm{Hf}, \mathrm{Yb}, \mathrm{Ta}, \mathrm{Y}, \mathrm{U}$, and Th are reported in the Table 2.

\subsection{Isotope analysis}

\subsection{1 - U-Pb SHRIMP Method}

CL and SEM Images of the zircons were obtained using an electron microscope (Jeol JSM 5800). The zircons were first analyzed for U-Pb with SHRIMP and then with LA-MC- 
ICP-MS for Lu-Hf. For Lu-Hf the laser spot was driven to same site or on the same zircon phase analyzed by U-Pb zircon SHRIMP method.

In situ U-Pb SHRIMP II (Sensitive High-Resolution Ion Microprobe II) zircon analyses were carried out at the John de Later Centre at Curtin University of Technology, Perth, Australia. Hand-picked zircons were mounted in an epoxy disc with chips of the TEMORA zircon standard, ground and polished, microphotographed in transmitted and reflected light, and imaged using a scanning electron microscope (backscattered electrons and charged contrast images). The mount was then cleaned and gold-coated in preparation for SHRIMP analysis. Used standard for calculation correction was the BR-266 with ${ }^{206} \mathrm{~Pb} /{ }^{238} \mathrm{U}$ age of $559 \mathrm{Ma}$. Analytical methods and data treatment follow those described by Williams (1998) and Williams and Meyer (1998). The isotope data were reduced by means of SQUID 1.00 software and concordia diagrams were calculated with $2 \sigma$ confiability level of $95 \%$. (Ludwing, 2001).

\subsection{2- Lu-Hf LA-MC-ICPMS Method}

In situ Lu-Hf isotopic measurements were subsequently performed by the LA-MCICPMS method using a Thermo Finnigan Neptune Multicollector - ICPMS and a Photonmachines excimer laser ablation system at the Multi-usuários Laboratório of the Universidade Estadual do Rio do Janeiro. For this work we analyzed the masses 171, 173, 174, 175, 176, 177, 178 and 179 simultaneously in Faraday cups with axial faraday cup at the mass 176. Isotope data were acquired using static mode and determination of 50 cycles of $1.054 \mathrm{~s}$. All values were corrected for blank values. Laser operating conditions are Laser output power 5 to $6.5 \mathrm{~J} / \mathrm{cm}^{2}$, shot repetition rate $=10 \mathrm{~Hz}$ and laser spot of $40 \mu \mathrm{m}$. Gas input are coolant flow (Ar) $15 \mathrm{l} / \mathrm{min}$, auxiliary flow (Ar) $0.7 \mathrm{l} / \mathrm{min}$, carrier (Ar) flow $0.75 \mathrm{l} / \mathrm{min}$ in the MC-ICP-MS and $0.22(\mathrm{Ar})$ and $0.55 \mathrm{l} / \mathrm{min}(\mathrm{He})$ in the Laser. Data were normalised to ${ }^{179} \mathrm{Hf} /{ }^{177} \mathrm{Hf} 50.7325$, using an exponential correction for mass bias.

Isotopic ratios and inter-element fractionation were evaluated by interspersing the GJ1 standard zircon (GEMOC, Macquarie University) on every set of 12 zircon samples (spots), eight unknow samples, two Mud Tank, and two 91500. To correct the isobaric interferences of $\mathrm{Lu}$ and $\mathrm{Yb}$ isotopes of mass 176 , the isotopes ${ }^{171} \mathrm{Yb},{ }^{173} \mathrm{Yb}$ and ${ }^{175} \mathrm{Lu}$ were simultaneously monitored during each analysis. The ${ }^{176} \mathrm{Lu}$ and ${ }^{176} \mathrm{Yb}$ were calculated using a ${ }^{176} \mathrm{Lu} /{ }^{175} \mathrm{Lu}$ ratio 
of 0.026549 and $a^{173} \mathrm{Yb} /{ }^{171} \mathrm{Yb}$ ratio of 1.123456 (Chu et. al. 2002) and Thirwall and Walder (1995), respectively. The correction for the instrumental mass bias used an exponential law and a ${ }^{179} \mathrm{Hf} /{ }^{177} \mathrm{Hf}$ value of 0.7325 (Patchett et al., 1981) to correct the Hf isotopic ratios.

During the course of the analyses the GJ-1 standard yielded ${ }^{176} \mathrm{Hf} /{ }^{177} \mathrm{Hf}$ ratio of $0.281994 \pm 17(\mathrm{n}=6.2 \mathrm{SD})$, which is almost identical to the recommended value by Morel et al. (2008) of $0.282000 \pm 5$. To evaluate the accuracy and precision of the laser-ablation results is reports here by the analyses of two zircon standards as unknown sample, 91500 and Mudtank, which yielded ${ }^{176} \mathrm{Hf} /{ }^{177} \mathrm{Hf}$ ratio of $0.282291 \pm 18$ and $0.282467 \pm 17$ similar to those suggested by Woodhead \& Hergt (2005). Chemale et al. (2011) described the detailed analytical methods and data treatment for the Lu-Hf method.

\section{5-LOCAL GEOLOGY AND PETROGRAPHY}

The mapped area covers $80 \mathrm{~km}^{2}$ and consists, in its central part, by a syenogranitic intrusion which it is gray to pale pink reaching reddish tones when oxidized or whitish when argillized. This intrusion is here included in Matupá Intrusive Suite (MIS), also informally called Matupá from Trairão. The MIS intrudes rocks of the Nhandu Intrusive Suite whereas mafic dykes and felsic cut both units. The geology of the area and the samples collected and the location of two gold mines in the off time are shown in the Figure 03 (E, F).

\section{1- Biotite Monzogranite (Nhandu Intrusive Suite)}

The biotite monzogranite outcrops in the southern and northern part of the area being cut by fine syenogranite Matupá. It occurs as pink to whitish gray, medium to coarse texture inegranular rock, composed of quartz, potassium feldspar, plagioclase, biotite and rarely hornblende. Under the microscope xenomorphic to hypidiomorphic texture is recognized and constituted by the following proportions of $35 \%$ quartz, $30 \%$ plagioclase; $25 \% \mathrm{~K}$-feldspar and $10 \%$ biotite + hornblende + opaque. Zircon and apatite occur as accessory minerals, and chorite is the most commun secondary mineral. Locally were found enclaves of intermediate composition. Under the microscope these enclaves consist of $60 \%$ plagioclase, $20 \%$ quartz, $15 \%$ alkali feldspar and $5 \%$ opaques.

Aplite dikes of granitic composition with fine graphic texture differs from granite by the size of grains of feldspar smaller than $1 \mathrm{~mm}$ of hydrothermal alteration. Dikes of diabase 
shows texture sub-ophitic to ophitic texture and are fine-grained holocrystalline, containing sometimes phenocrysts of plagioclase, orthopyroxene and clinopyroxene (augite), and as accessories occur opaque minerals and acicular apatite. The secondary minerals were identified as calcite and amphibole, alteration product of pyroxene.

\section{2- Biotite Syenogranite (Matupá Intrusive Suite)}

The $40 \mathrm{~km}^{2}$ large is represented by fine-grained granitic rocks of gray to pale pink reaching reddish tones when oxidized or whitish when argillized. In the field includes in its southwestern portion mineralization disseminated in breccias and veins, associated with hydrothermal alteration. Sulfides and metal oxides are arranged in disseminated form the rock in boxwork type or associated with late quartz veins structures. This area was extensively mined and the deposits known as Trairão and Chumbo Grosso deposits (Figure 3, E,F). Portions of preserved granite were observed under a microscope which can observe an inequigranular texture with fine-grained, low content of mafic minerals (leucogranite).

Anhedral, subedric alkali feldspar with dimensions of 0.5 to $1.0 \mathrm{~mm}$ show twinning (combining albite and pericline) and micro-perthitic intergrowth. The potassium feldspar is partially replaced by white mica.

Quartz occurs in recrystallized aggregates with slightly undulating extinction, with knurled contact with other minerals. The grains measure $1.5 \mathrm{~mm}$ to $0.2 \mathrm{~mm}$ in the recrystallized portions, generating subgrains. Graphic intergrowth texture with alkali feldspar aand quartz is also recognized.

Subhedral plagioclase with local polysynthetic twins (according to the law of albite), measuring 0.5 to $1.3 \mathrm{~mm}$, are zoned and intensely saussuritizated, locally occurring small inclusions of accessory minerals in the core of the crystal. A mimerkític texture is also recognized. The brownish biotite has lamellar habit (up to $0.4 \mathrm{~mm}$ ) and occurs mainly in the grain boundaries and is often partially or completely converted into chlorite. As accessory minerals were observed rutile, apatite, zircon and small proportions of magnetite. As a secondary mineral assembly is chlorite, sericite and epidote.

\section{6- LITHOGEOCHEMISTRY}

The chemical analyzes for major, trace, and Rare Earth Elements (RREs) are shown in Table - 01. The TRBY - 40A ; TRBY- 45; TRBY- 60A ; TRBY- 60C; TRBY -05 ; TRBY- 
32F and TRBY -32G samples belong to the Nhandu Intrusive Suite and are represented by blue cross. Matupá Intrusive Suite samples (TRBY -25, 30, 12, 26 and 27) are represented by red circles. Two samples, TRBY -13 and TRBY -17 , show geochemical anomalous patterns due to changes during the hydrothermal alteration. These samples were not used in the classification and discrimination diagrams.

In discrimination diagram (Peccerilo and Taylor, 1976), the rocks of both units display ferrous affinities, fall in the calc-alkaline field to shoshonite, while with respect to the parameter settings of Shand (1943) both samples Nhandu Granite as Matupá are metaluminous the peraluminous slightly, ferrous. A single sample (TRBY-5) is plotted in the field of peraluminous rocks with A/ACNK close to 1.4 (Figure 04).

Showing the ETR patterns for Nhandu and Matupá granites normalized to chondrite (Nakamura, 1974) with the fractionation of light REE and negative Eu anomaly. The total content of REE is moderate, which it is common to I-type, calc-alkaline granite. In the multi- 


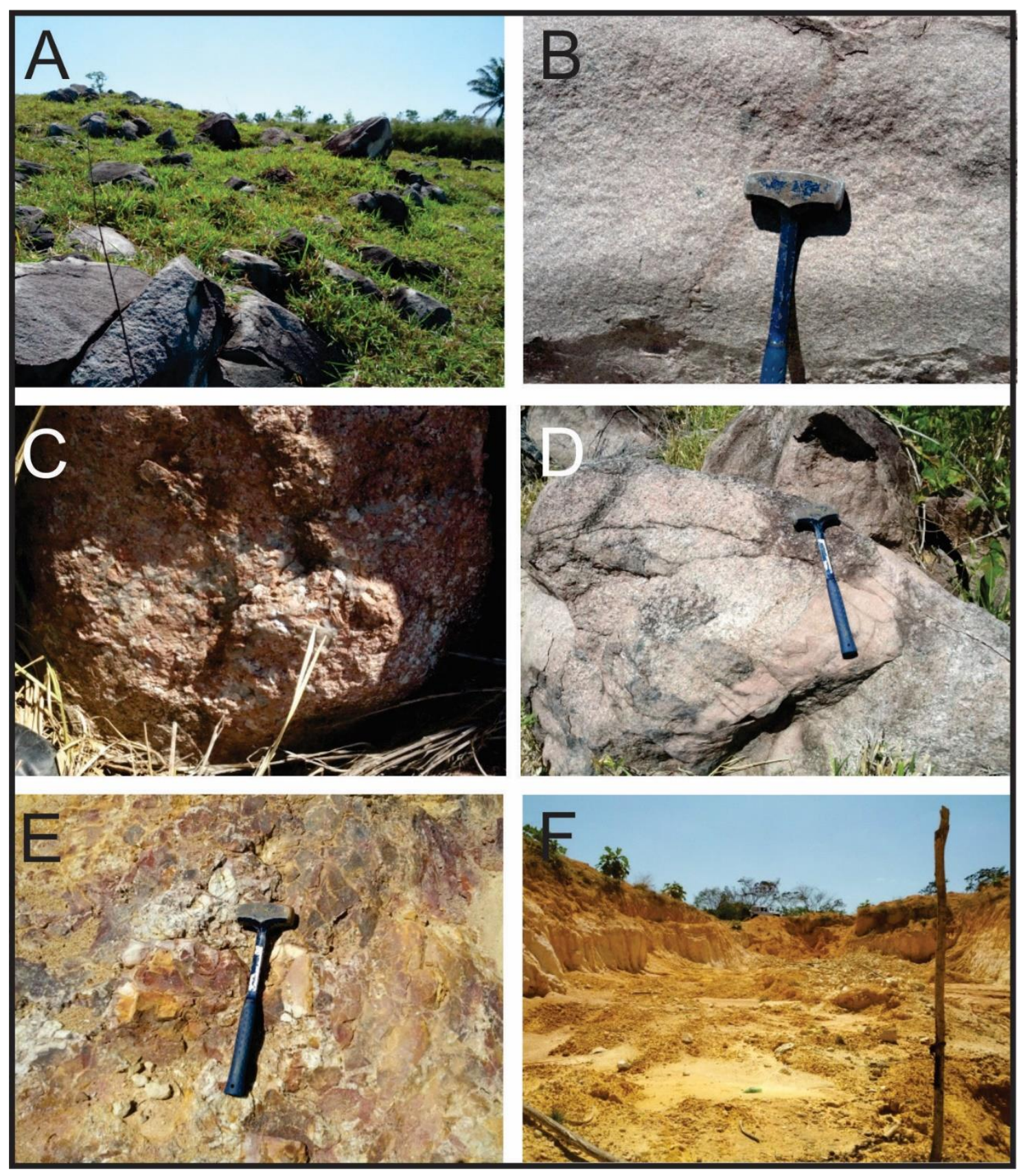

IV Figure 03 - (A) Exposures of granites blocks in the field; (B) Fine-grained syenogranite; (C) biotite syenogranite; (D) Aplite Dyke cutting the biotite monzogranite; (E) Hyddrothermal Breccia in the gold deposit; $(F)$ Gold-digging deactivated. 


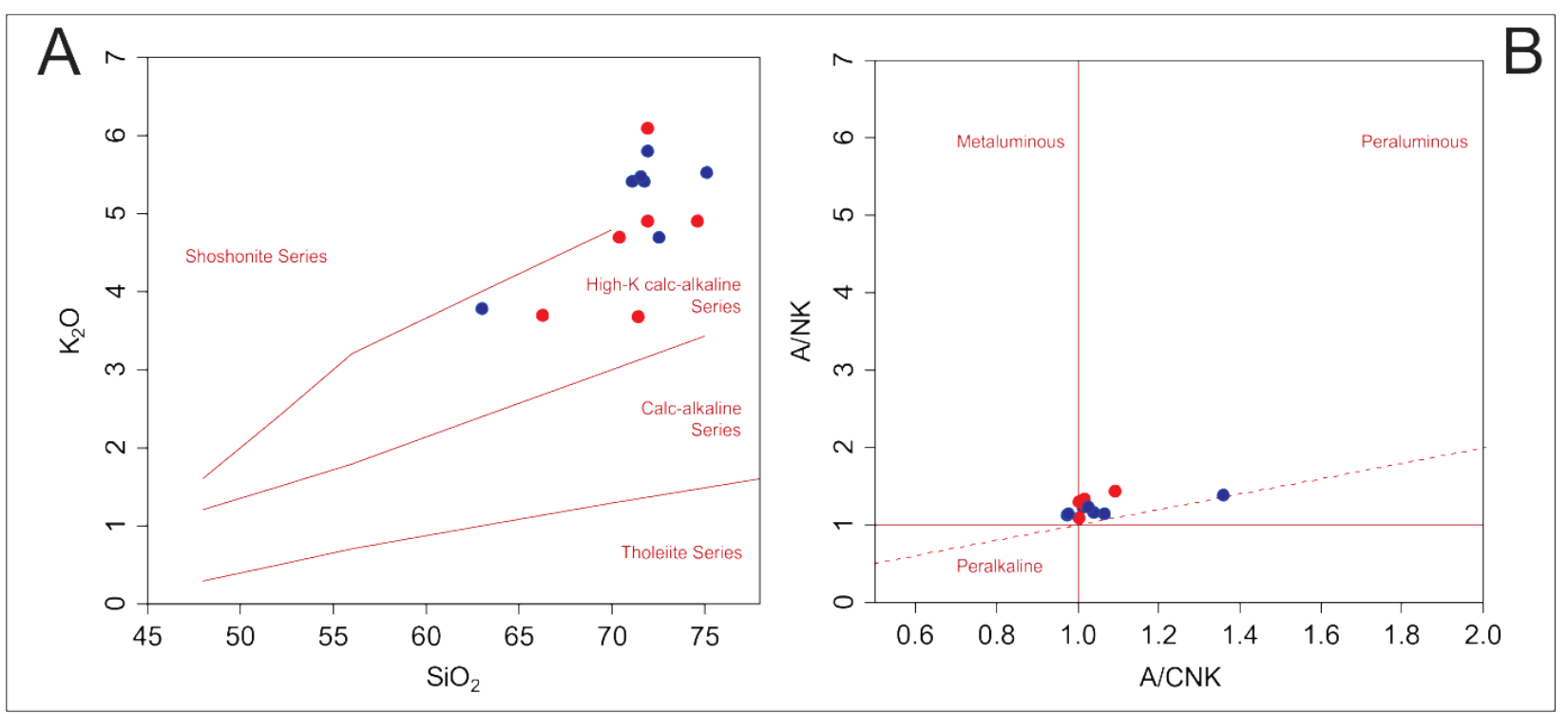

IV Figure 04 - Geochemical classification diagram and magmatic series. (A) $\mathrm{SiO}_{2}$ versus $K_{2} O$ (Peccerillo \& Taylor 1976, 1989); (B) ANK versus ACNK diagram of Maniar \& Piccoli (1989) with peraluminous index of Shand (1943). Nhandu Intrusive Suite (blue) and Matupá Intrusive Suite (red).

element diagram normalized to Chondrite (Thompson, 1982) the analysed samples display negative anomaly of $\mathrm{Ba}, \mathrm{Rb}, \mathrm{Nb}, \mathrm{Ta}, \mathrm{Sr}, \mathrm{P}, \mathrm{Th}$ and $\mathrm{Ti}$ (Figure 05).

The samples plotted on tectonic discrimination diagram proposed by Pearce et al. (1984 and Pearce 1996), Y versus Nb are not very conclusive, but show a tendency for both units similar source of magmatic arc, this trend are confirmed using diagram of Harris et al. (1986) fall in the arc volcanic field (Figure 06).

The geochemical pattern of the analyzed samples shows that both groups have similar sources, K-rich- calc- alkaline composition, probably derived from juvenile sources of magmatic arc environment with mixing of crustal material in different proportions. Negative anomalies of $\mathrm{Nb}$ and $\mathrm{Ta}$ are common in calc-alcaline granites, especially those formed in subduction environments. Plagioclase fractionation is shown in negative $\mathrm{Eu}$ anomaly and fractionation of apatite and titanomagnetite appear to have occurred during the process of magmatic crystallization 

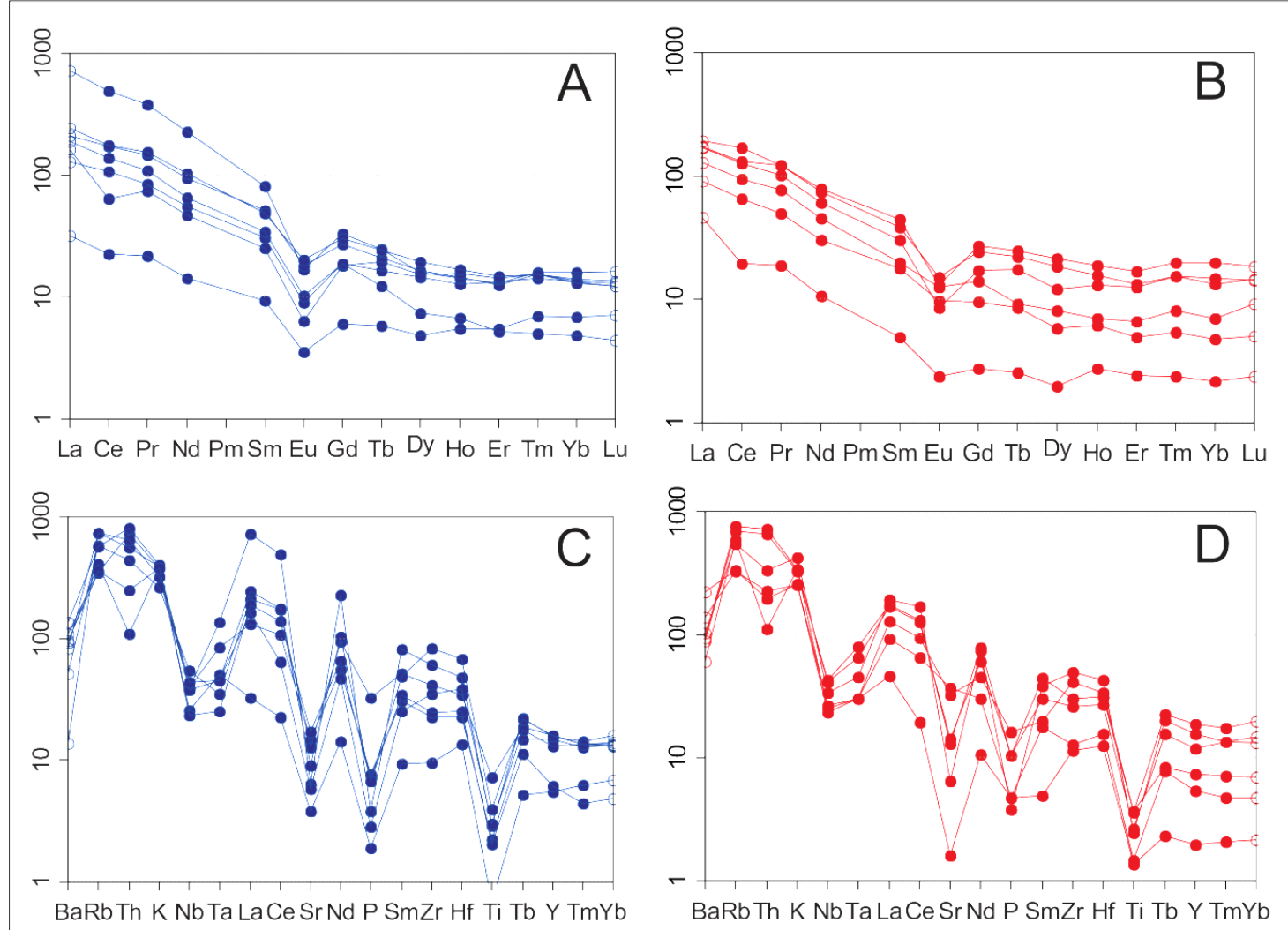

IV Figure $05-(A-B)$ Multielement diagram for analyzed granite samples normalized to chondrite (Thompson, 1982). (C-D) REE patterns normalized to chondrite after Nakamura *1974). Nhandu Intrusive Suite (blue) and Matupá Intrusive Suite (red).

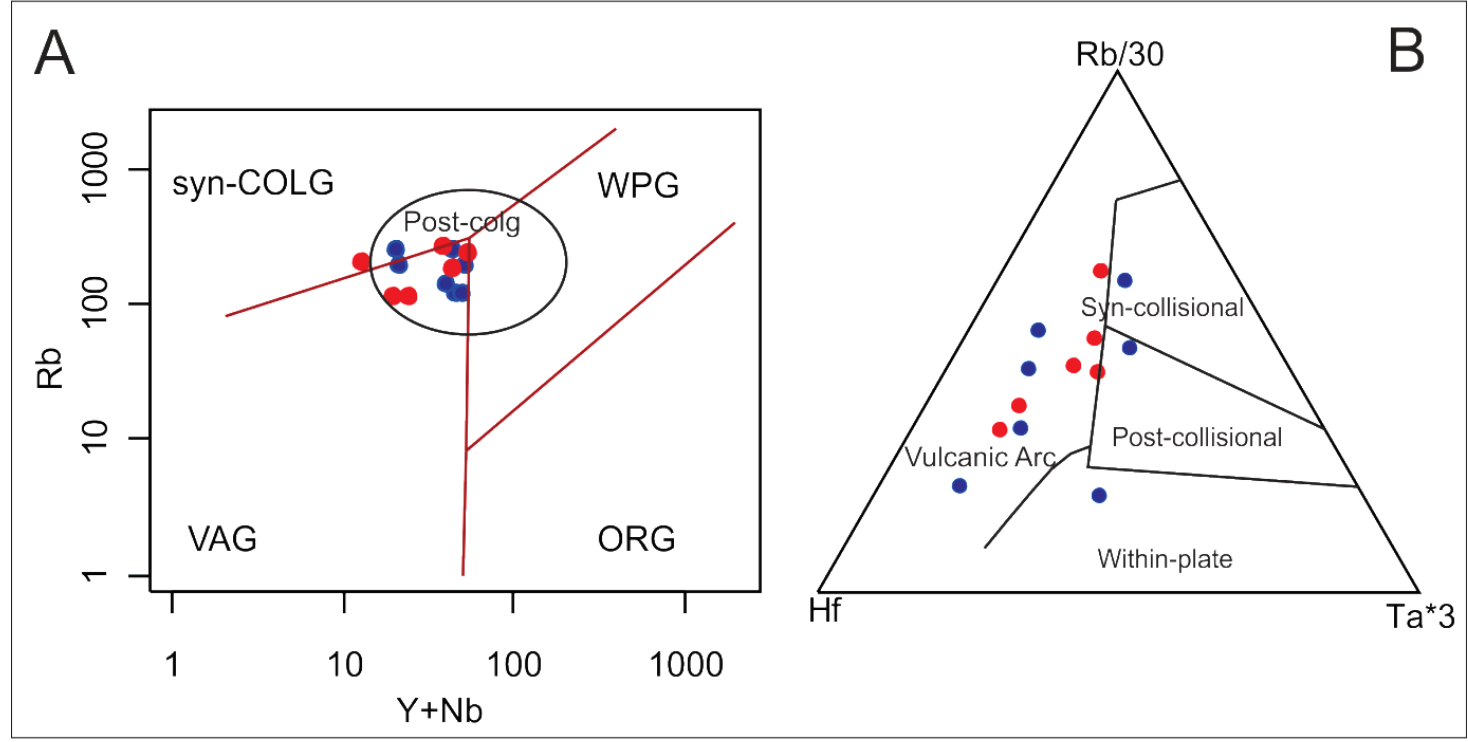

IV Figure 06- (A) Tectonic setting discrimination diagram with $\mathrm{R} b$ versus $Y+N b$ (Pearce et al, 1984, Pearce 1996 ); (B) Tectonic discrimination diagram, Harris et al. (1986). Nhandu Intrusive Suite (blue) and Matupá Intrusive Suite (red). 


\begin{tabular}{|c|c|c|c|c|c|c|c|c|c|c|c|c|c|}
\hline \multirow[b]{2}{*}{ Sample } & \multicolumn{7}{|c|}{$\begin{array}{c}\text { Nhandu Intrusive Suite } \\
\text { Biotite Monzogranite }\end{array}$} & \multicolumn{6}{|c|}{$\begin{array}{l}\text { Matupá Intrusive Suite } \\
\text { Biotite Syenogranite }\end{array}$} \\
\hline & $\begin{array}{c}\text { TRBY } \\
\text { 40A }\end{array}$ & $\begin{array}{c}\text { TRBY } \\
12\end{array}$ & $\begin{array}{c}\text { TRBY } \\
\text { 60A }\end{array}$ & $\begin{array}{c}\text { TRBY } \\
\text { 60C }\end{array}$ & $\begin{array}{l}\text { TRBY } \\
05\end{array}$ & $\begin{array}{c}\text { TRBY } \\
\text { 32G }\end{array}$ & $\begin{array}{c}\text { TRBY } \\
\text { 32F }\end{array}$ & $\begin{array}{l}\text { TRBY } \\
25\end{array}$ & $\begin{array}{l}\text { TRBY } \\
30\end{array}$ & $\begin{array}{l}\text { TRBY } \\
45\end{array}$ & $\begin{array}{c}\text { TRBY } \\
20\end{array}$ & $\begin{array}{c}\text { TRBY } \\
26\end{array}$ & $\begin{array}{c}\text { TRBY } \\
27\end{array}$ \\
\hline $\mathrm{SiO}_{2}$ & 71.7 & 66.24 & 72.63 & 63.02 & 71.43 & 71.26 & 71.97 & 71.44 & 71.88 & 75.07 & 75.96 & 71.88 & 70.38 \\
\hline $\mathrm{TiO}_{2}$ & 0.3 & 0.38 & 0.23 & 0.74 & 0.31 & 0.41 & 0.21 & 0.15 & 0.27 & 0.07 & 0.14 & 0.25 & 0.37 \\
\hline $\mathrm{Al}_{2} \mathrm{O}_{3}$ & 13.64 & 15.74 & 13.4 & 15.58 & 14.22 & 13.55 & 13.65 & 15.06 & 13.89 & 12.89 & 15.48 & 13.27 & 13.86 \\
\hline $\mathrm{Fe}_{2} \mathrm{O}_{3}$ & 3.59 & 4.4 & 3.01 & 5.59 & 2.66 & 3.61 & 2.88 & 2.2 & 2.47 & 2.19 & 1.11 & 3.54 & 3.66 \\
\hline $\mathrm{MnO}$ & 0.06 & 0.08 & 0.06 & 0.21 & 0.05 & 0.11 & 0.04 & 0.06 & 0.06 & 0.03 & 0.01 & 0.07 & 0.07 \\
\hline $\mathrm{MgO}$ & 0.28 & 0.99 & 0.4 & 2.3 & 0.4 & 0.33 & 0.46 & 0.45 & 0.48 & 0.08 & 0.31 & 0.15 & 0.58 \\
\hline $\mathrm{CaO}$ & 0.51 & 2.11 & 1.35 & 1.35 & 0.18 & 1.2 & 1.26 & 1.9 & 1.49 & 0.78 & 0.02 & 0.72 & 1.83 \\
\hline $\mathrm{Na}_{2} \mathrm{O}$ & 3.64 & 4.66 & 3.47 & 5.78 & 2.55 & 3.53 & 2.89 & 3.88 & 3.43 & 3.04 & 0.12 & 3.25 & 3.31 \\
\hline $\mathrm{K}_{2} \mathrm{O}$ & 5.44 & 3.7 & 4.69 & 3.79 & 5.5 & 5.46 & 5.81 & 3.69 & 4.92 & 5.55 & 4.75 & 6.11 & 4.7 \\
\hline $\mathrm{P}_{2} \mathrm{O}_{5}$ & 0.04 & 0.17 & 0.08 & 0.34 & 0.03 & 0.07 & 0.07 & 0.05 & 0.11 & 0.02 & 0.06 & 0.04 & 0.11 \\
\hline LOI & 0.5 & 1.2 & 0.5 & 1.1 & 2.5 & 0.2 & 0.5 & 0.9 & 0.8 & 0.2 & 2 & 0.5 & 0.9 \\
\hline SUM & 99.74 & 99.68 & 99.81 & 99.8 & 99.85 & 99.73 & 99.77 & 99.8 & 99.83 & 99.92 & 99.91 & 99.78 & 99.79 \\
\hline $\mathrm{Ba}(\mathrm{ppm})$ & 663 & 1533 & 566 & 357 & 633 & 758 & 942 & 946 & 649 & 95 & 179 & 710 & 617 \\
\hline $\mathrm{Rb}$ & 122.2 & 116.4 & 255.2 & 202.5 & 143.2 & 126.1 & 197.4 & 114.5 & 267.8 & 257.4 & 146.6 & 191.4 & 244.6 \\
\hline $\mathrm{Sr}$ & 74.4 & 381.9 & 173.4 & 151 & 67.7 & 105.9 & 202.9 & 437.9 & 168.7 & 44.6 & 14 & 75.8 & 154.3 \\
\hline $\mathrm{Zr}$ & 414.1 & 283.5 & 154.5 & 281.6 & 240.2 & 565.8 & 169.5 & 87.7 & 177 & 64.5 & 75.7 & 340.2 & 204.7 \\
\hline $\mathrm{Nd}$ & 142 & 28.5 & 34.6 & 59.4 & 29.6 & 65.1 & 41.3 & 19.1 & 38.5 & 9 & 17.9 & 46.4 & 49.4 \\
\hline $\mathrm{Y}$ & 30.9 & 14.7 & 29.4 & 31.9 & 25.7 & 31.9 & 12.2 & 10.7 & 23.7 & 11 & 6.7 & 30.9 & 37.6 \\
\hline $\mathrm{Ga}$ & 19.4 & 16.6 & 14.9 & 15.8 & 18.5 & 16 & 12.2 & 15.8 & 15.8 & 15.4 & 14.7 & 15.1 & 15.7 \\
\hline $\mathrm{Se}$ & 0 & 0.5 & 0 & 0 & 0 & 0.5 & 0.5 & 0 & 0 & 0 & 0.5 & 0.5 & 0.5 \\
\hline $\mathrm{Th}$ & 30.2 & 8.3 & 26.9 & 18.5 & 4.6 & 10.4 & 34.1 & 9.6 & 30.1 & 23.4 & 0.9 & 13.9 & 27.5 \\
\hline $\mathrm{U}$ & 3.2 & 1.7 & 8 & 5.8 & 1.6 & 1 & 1.2 & 1.4 & 7.4 & 23.2 & 0.1 & 1.9 & 4 \\
\hline $\mathrm{V}$ & 36 & 27 & 17 & 31 & 16 & 8 & 18 & 13 & 21 & 11 & 10 & 8 & 34 \\
\hline $\mathrm{Co}$ & 4.2 & 4.6 & 2.6 & 10.5 & 2.4 & 1.6 & 2.7 & 4.1 & 3.2 & 1.1 & 1 & 1.5 & 5.2 \\
\hline
\end{tabular}




\begin{tabular}{|c|c|c|c|c|c|c|c|c|c|c|c|c|c|}
\hline $\mathrm{Ni}$ & 52 & 20 & 0 & 28 & 0 & 20 & 20 & 0 & 0 & 0 & 20 & 20 & 20 \\
\hline $\mathrm{Cu}$ & 24.7 & 10.7 & 19.7 & 14.3 & 11.2 & 16.8 & 12 & 6.4 & 9.4 & 27.4 & 14 & 14.9 & 32.4 \\
\hline $\mathrm{Zn}$ & 52 & 26 & 59 & 165 & 54 & 57 & 29 & 29 & 32 & 13 & 1 & 73 & 45 \\
\hline $\mathrm{La}$ & 238.8 & 42.5 & 42.8 & 69.4 & 53.7 & 80.2 & 62.4 & 30.2 & 55.5 & 10.6 & 19.4 & 63.2 & 57.4 \\
\hline $\mathrm{Ce}$ & 424.1 & 81.5 & 91.7 & 148.7 & 55.4 & 151.3 & 118.7 & 56.4 & 108.7 & 19.6 & 50.8 & 145.3 & 112.5 \\
\hline $\mathrm{Nb}$ & 13.7 & 9.2 & 13.7 & 19 & 13.1 & 15.2 & 8.1 & 8.2 & 14.4 & 8.9 & 6.6 & 11.8 & 14.9 \\
\hline $\mathrm{Sm}$ & 16.34 & 4.03 & 6.19 & 10.47 & 5.07 & 9.81 & 6.96 & 3.61 & 6.1 & 1.89 & 2.97 & 7.8 & 8.93 \\
\hline$\overline{\mathrm{Eu}}$ & 1.29 & 0.96 & 0.69 & 1.4 & 0.49 & 1.54 & 0.79 & 0.75 & 0.66 & 0.27 & 0.75 & 1.16 & 1 \\
\hline $\mathrm{Gd}$ & 9.19 & 3.84 & 5.12 & 7.42 & 5.01 & 8.37 & 5.21 & 2.63 & 4.7 & 1.66 & 2 & 6.7 & 7.4 \\
\hline Dy & 5.48 & 2.75 & 4.94 & 5.61 & 5.23 & 6.65 & 2.52 & 1.97 & 4.15 & 1.64 & 1.45 & 6.32 & 7.35 \\
\hline $\mathrm{Er}$ & 2.89 & 1.47 & 2.96 & 2.81 & 3.17 & 3.33 & 1.16 & 1.11 & 2.81 & 1.23 & 0.6 & 2.96 & 3.76 \\
\hline $\mathrm{Yb}$ & 3.47 & 1.54 & 2.91 & 3.08 & 2.95 & 2.85 & 1.05 & 1.03 & 2.89 & 1.5 & 0.54 & 3.23 & 4.31 \\
\hline $\mathrm{Lu}$ & 0.55 & 0.31 & 0.42 & 0.46 & 0.44 & 0.42 & 0.15 & 0.17 & 0.49 & 0.24 & 0.07 & 0.48 & 0.62 \\
\hline $\begin{array}{c}\mathrm{FeO} / \mathrm{FeOt} \\
+\mathrm{MgO}\end{array}$ & 1.18 & 1.89 & 1.3 & 3.2 & 1.3 & 1.23 & 1.36 & 1.35 & 1.38 & 0.98 & 1.21 & 1.05 & 1.48 \\
\hline $\mathrm{K}_{2} \mathrm{O} / \mathrm{Na}_{2} \mathrm{O}$ & 1.49 & 0.79 & 1.35 & 0.65 & 2.15 & 1.54 & 2.01 & 0.95 & 1.43 & 1.82 & 39.58 & 1.88 & 1.41 \\
\hline $\mathrm{A} / \mathrm{CNK}$ & 1.42 & 1.5 & 1.4 & 1.42 & 1.72 & 1.32 & 1.37 & 1.59 & 1.41 & 1.37 & 3.16 & 1.31 & 1.4 \\
\hline $\mathrm{A} / \mathrm{NK}$ & 1.5 & 1.88 & 1.64 & 1.62 & 1.78 & 1.5 & 1.56 & 1.98 & 1.66 & 1.5 & 3.17 & 1.41 & 1.73 \\
\hline$(\mathrm{La} / \mathrm{Yb}) \mathrm{n}$ & 46.8 & 1.6 & 1.2 & 1.7 & 1.3 & 1.4 & 3.8 & 2.7 & 1.7 & 36.7 & 1.6 & 1.8 & 0.6 \\
\hline $\mathrm{Eu} / \mathrm{Eu}^{*}$ & 0.32 & 0.75 & 0.37 & 0.49 & 0.3 & 0.52 & 0.14 & 0.74 & 0.38 & 0.47 & 0.94 & 0.49 & 0.38 \\
\hline $\mathrm{Rb} / \mathrm{Sr}$ & 1.64 & 0.3 & 1.47 & 1.34 & 2.11 & 1.19 & 0.97 & 0.26 & 1.58 & 5.77 & 10.47 & 2.53 & 1.58 \\
\hline $\mathrm{Rb} / \mathrm{Ba}$ & 0.18 & 0.07 & 0.45 & 0.56 & 0.22 & 0.16 & 0.2 & 0.12 & 0.41 & 2.7 & 0.81 & 0.26 & 0.39 \\
\hline $\mathrm{CaO} / \mathrm{Na}_{2} \mathrm{O}$ & 0.14 & 0.45 & 0.38 & 0.23 & 0.07 & 0.33 & 0.43 & 0.48 & 0.47 & 0.25 & 0.16 & -0.22 & 0.55 \\
\hline $\mathrm{Al}_{2} \mathrm{O}_{3} / \mathrm{TiO}_{2}$ & 45.4 & 41.4 & 58.2 & 21 & 45.8 & 33 & 65 & 100.4 & 51.4 & 184.1 & 110.5 & 53 & 37.4 \\
\hline $\mathrm{T} \mathrm{Zr}\left({ }^{\circ} \mathrm{C}\right)$ & 876.8 & 823.6 & 779.9 & 808.8 & 849.4 & 896.8 & 788.4 & 738.5 & 791 & 714.8 & 789.7 & 849.9 & 800.4 \\
\hline
\end{tabular}

IV Table 01 - Geochemical analyses of rocks from Nhandu Intrusive Suite and Matupá Intrusive Suite at the Novo Mundo region (MT). Oxides in

$\%$ and trace and rare earth elements in ppm. 


\section{6- ISOTOPE GEOLOGY}

\section{1- Zircon Characteristics}

Zircon is a mineral very common accessory in granitic rocks and is commonly used to $\mathrm{U}-\mathrm{Pb}$ geochronological study due to their behavior as a substantially closed system and for introducing high amounts of $\mathrm{U}$ and $\mathrm{Th}$ and a small (or zero) amount of common $\mathrm{Pb}$. The morphology of the grains of the zircon grains of Nhandu Granite samples are shown in Figure 06 and Matupá Granite in Figure 07. The crystals are elongated, prismatic, commonly bipyramid and pale brown. Images of backscattered electrons (BSE) of the internal structures reveal concentric zonation (the core to the edge) marked by metamític change. The grains of Suite Matupá differ from Nhandu granite crystals to be more homogeneous and smaller. The metamictization process is more effective in the zircon grains of the Nhandu granite.
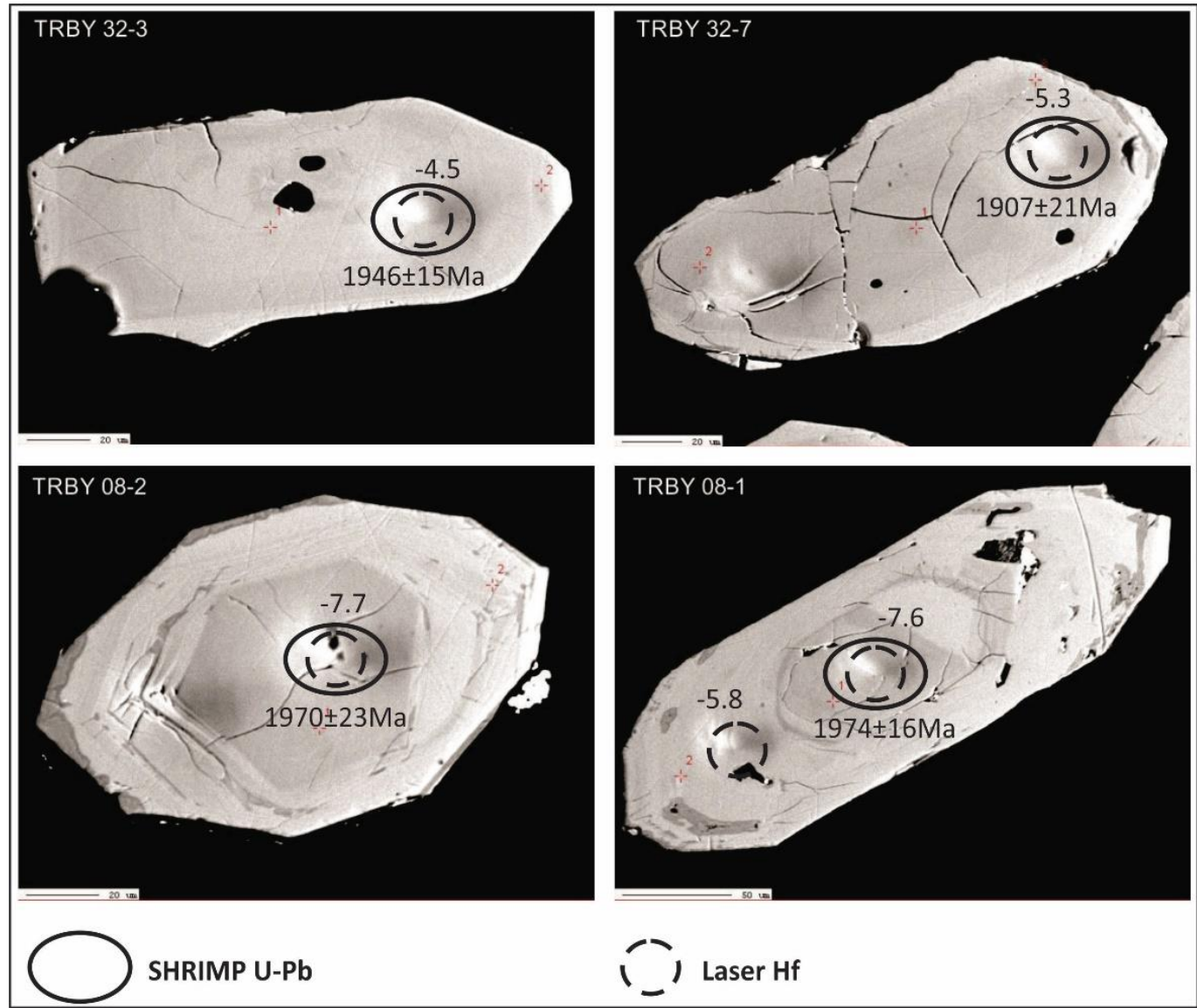

IV Figure 07- BSE image of first population of zircon crystals, TRBY- 32 and TRBY-08 samples, showing their morphology. Red points indicate the locations analyzed by WDS. 

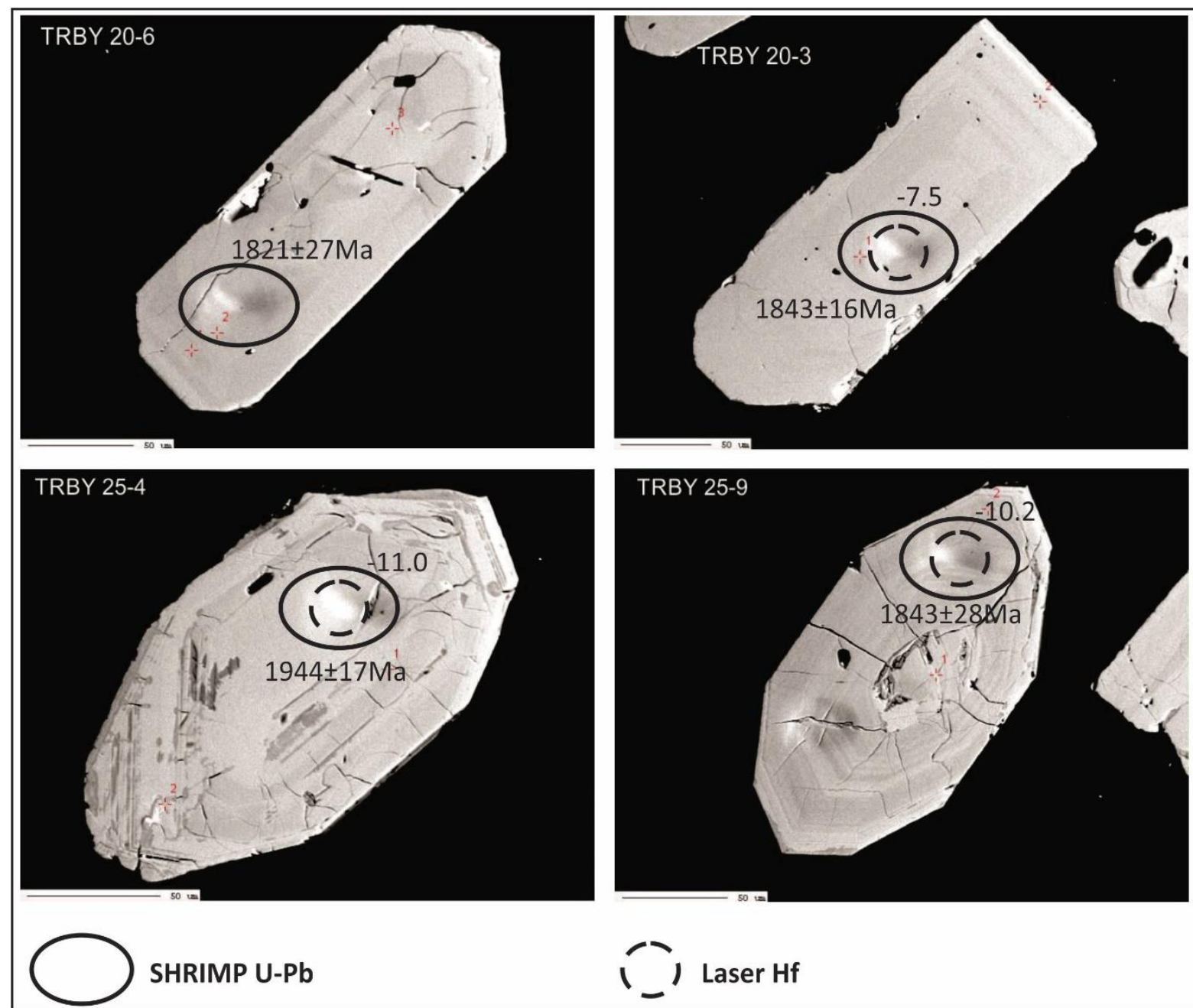

IV Figura 08 - BSE image of seconf population of zircon crystals, TRBY- 20 and TRBY- 25 samples, showing their morphology. Red points indicate the locations analyzed by WDS.

\section{2 - Zircon mineral chemistry}

Six samples have been analyzed and present some differences between the Nhandu Granite and Matupá Granite samples. The chemical analyzes for major and trace, and elements are shown in Tables 02 and 03 . The 4 analyzed samples of Nhandu Granite present $\mathrm{Th} / \mathrm{U}$ ratios ranging from 0.36-0.83 (TRBY-32), 0.34-082 (TRBY-32 F), 0.22-0.66 (TRBY-32 G) and 0.195.48 (TRBY-08). In the samples occur few zircons with very low ratio of $\mathrm{Th} / \mathrm{U}$ (0.02 to 0.16$)$, at zircon border. The Hf values are somewhat consistent for all samples ranging $0.82 \%$ to 1.72 $\%$ with mean value of $1.24 \%$. The mean values for analyzed zircons of Nhandu Granite are for $\mathrm{Ta}=920.9 \mathrm{ppm}, \mathrm{Yb}=327.6 \mathrm{ppm}, \mathrm{Y}=1537.8 \mathrm{ppm}, \mathrm{U}=431.3 \mathrm{ppm}, \mathrm{Th}=361.4, \mathrm{ZrO}_{2}=64.5 \%$ 
and $\mathrm{SiO}_{2}=32.8 \%$. The 2 analyzed samples of Matupá Granite show Th/U ratios from 0.2 to 0.87 and 1.1 to 5.2 (TRBY-25) and from 0.25 to 0.62 (TRBY-20). One zircon present ratio of 0.058 and another one of 2.32 in ths last sample. The Hf values are somewhat consistent for all samples ranging $0.90 \%$ to $1.71 \%$ with mean value of $1.04 \%$. The mean values for analyzed zircons of Nhandu Granite are for Ta=290.8 ppm, Yb=937.6 ppm, Y = 1497.4 ppm, U=228.5 ppm, $\mathrm{Th}=144.6, \mathrm{ZrO}_{2}=65.7 \%$ and $\mathrm{SiO}_{2}=32.5 \%$.

\section{3 - U-Pb and Lu-Hf zircon dating}

The U-Pb zircon geochronological results of six samples are shown in the Table 02 (Nhandu Intrusive Suite) and Table 03 (Matupá Intrusive Suite). Three samples of Nhandu Intrusive Suite have been dated. The results range from $1946 \pm 21 \mathrm{Ma}$ ( 8 zircon grains), $1969 \pm$ 7.3Ma (9 zircon grains) and 1954.5 $\pm 4.5 \mathrm{Ma}$ (6 grains), (Figure 09). Three samples of Matupá Intrusive Suite have been dated. The results range from $1837 \pm 13 \mathrm{Ma}$ ( 3 zircon grains), $1851 \pm$ $12 \mathrm{Ma}$ (11 zircon grains) and 1878.4 $\pm 7.5 \mathrm{Ma}$ (8 zircon grains) (Figure 10).

Four samples were analyzed by the Lu-Hf zircon method. The results are shown in the Tables 04 and 05. The isotopic ratios of ${ }^{176} \mathrm{Lu} /{ }^{177} \mathrm{Hf}$ and ${ }^{176} \mathrm{Hf} /{ }^{177} \mathrm{Hf}$ of four samples from Trairão region, ranging from 0.0005 to 0.0037 and 0.281291 to 0.281450 , respectively, this is evidence of low radiogenic growth of ${ }^{176} \mathrm{Hf}$. Three of the four samples display intervals with a limited number of epsilon Hf values in the crystallization time, EHf ( $\mathrm{t}$ ) values from -3.1 to -6.5 and Hf model age from 2.68 to $2.84 \mathrm{Ga}$. Only one sample has higher negative $\mathcal{E H f}(\mathrm{t})$ values between -7.9 and -9.3 and Hf model age from 2.86 to 2.93 Ga (sample TRBY-25).

\section{4-WR Sm-Nd isotopes}

Whole rock Sm-Nd analyses carried out in 5 samples of Nhandu Granite and 3 samples of Matupá Granite. The results are shown in the Table 08. The analyzed granitcs rocks have Sm-Nd fractionation compatible to the crustal values, ranging from -0.38 to -0.59 . Nhandu granitic samples display eNd (t) values grom -0.12 to -6.42 and $\mathrm{Nd}$ model ages from 2.27 to $2.54 \mathrm{Ga}$, whereas the Matupá granitic rocks show eNd (t) between +0.05 and -3.44 and $\mathrm{Nd}$ model ages 2.25 and $2.36 \mathrm{Ga}$. 


\begin{tabular}{|c|c|c|c|c|c|c|c|c|c|c|}
\hline Sample & $\begin{array}{c}\mathrm{Yb} \\
\mathrm{ppm}\end{array}$ & $\begin{array}{c}\mathrm{Ta} \\
\mathrm{ppm}\end{array}$ & $\begin{array}{c}\mathrm{Hf} \\
\mathrm{ppm}\end{array}$ & $\begin{array}{c}\mathrm{Y} \\
\mathrm{ppm}\end{array}$ & $\begin{array}{c}\mathrm{U} \\
\mathrm{ppm}\end{array}$ & $\begin{array}{c}\text { Th } \\
\mathrm{ppm}\end{array}$ & $\begin{array}{c}\mathrm{Zr} \\
\mathrm{ppm}\end{array}$ & $\begin{array}{l}\mathrm{ZrO}_{2} \\
\%\end{array}$ & $\begin{array}{l}\mathrm{SiO}_{2} \\
\%\end{array}$ & Th/U \\
\hline TRBY-08_1-N & 298.6 & 769.8 & 10226.6 & 433.1 & 114.6 & 26.4 & 483682 & 65.335 & 32.779 & 0.23 \\
\hline TRBY-08_1-B & 1826.6 & 384.9 & 12100.7 & 12378.6 & 1763.0 & 2697.9 & 450538 & 60.858 & 31.605 & 1.53 \\
\hline TRBY-08_2-N & 289.8 & 696.1 & 8242.4 & 2393.8 & 105.8 & 202.1 & 479558 & 64.778 & 32.540 & 1.91 \\
\hline TRBY-08_3-N & 500.6 & 999.1 & 11252.7 & 3110.4 & 167.5 & 632.7 & 478544 & 64.641 & 32.464 & 3.78 \\
\hline TRBY-08_6-N & 122.9 & 466.8 & 10336.9 & 771.7 & 52.9 & 105.5 & 479143 & 64.722 & 32.180 & 1.99 \\
\hline TRBY-08_6-B & 254.7 & 761.6 & 12287.2 & 204.7 & 458.4 & 105.5 & 484703 & 65.473 & 33.262 & 0.23 \\
\hline TRBY-08_4-B & 8.8 & 1244.8 & 12855.4 & 370.1 & 185.1 & 35.2 & 481883 & 65.092 & 33.301 & 0.19 \\
\hline TRBY-08_4-N & 263.5 & 851.7 & 10854.1 & 488.2 & 158.7 & 149.4 & 483904 & 65.365 & 33.124 & 0.94 \\
\hline TRBY-08_5-N & 693.8 & 1031.9 & 11507.1 & 2661.5 & 449.6 & 474.6 & 476212 & 64.326 & 32.555 & 1.06 \\
\hline TRBY-08_7-N & 219.5 & 1097.4 & 11049.2 & 944.9 & 97.0 & 43.9 & 480543 & 64.911 & 32.931 & 0.45 \\
\hline TRBY-08_7-B & 61.5 & 851.7 & 12092.2 & 165.4 & 52.9 & 290.0 & 478337 & 64.613 & 32.803 & 5.48 \\
\hline TRBY-32_1-N & 254.7 & 679.7 & 12041.3 & 1417.4 & 229.2 & 175.8 & 481239 & 65.005 & 32.987 & 0.77 \\
\hline TRBY-32_1-B & 588.4 & 614.2 & 14500.5 & 5653.8 & 1904.0 & 711.8 & 407030 & 54.981 & 29.694 & 0.37 \\
\hline TRBY-32_2-N & 228.3 & 450.4 & 13457.4 & 2094.6 & 669.9 & 325.2 & 473850 & 64.007 & 33.187 & 0.49 \\
\hline TRBY-32_2-B & 114.2 & 835.3 & 12965.6 & 456.7 & 335.0 & 87.9 & 477034 & 64.437 & 32.822 & 0.26 \\
\hline TRBY-32_3-N & 430.3 & 597.8 & 11837.8 & 2606.4 & 431.9 & 325.2 & 477270 & 64.469 & 32.875 & 0.75 \\
\hline TRBY-32_3-B & 61.5 & 712.5 & 13228.5 & 417.3 & 202.7 & 70.3 & 480365 & 64.887 & 32.613 & 0.35 \\
\hline TRBY-32_4-N & 281.0 & 843.5 & 12796.0 & 1716.6 & 608.2 & 290.0 & 476678 & 64.389 & 32.738 & 0.48 \\
\hline TRBY-32_4-B & 105.4 & 810.8 & 11201.8 & 708.7 & 44.1 & 96.7 & 480113 & 64.853 & 32.970 & 2.19 \\
\hline TRBY-32_6-B & 281.0 & 1081.0 & 10226.6 & 504.0 & 79.3 & 52.7 & 481290 & 65.012 & 32.684 & 0.66 \\
\hline TRBY-32_5-N & 289.8 & 769.8 & 8496.8 & 1779.6 & 158.7 & 105.5 & 478596 & 64.648 & 32.262 & 0.66 \\
\hline TRBY-32_9-N 1 & 219.5 & 884.5 & 12236.4 & 78.7 & 79.3 & 61.5 & 476982 & 64.430 & 32.291 & 0.78 \\
\hline TRBY-32_9-B & 474.2 & 933.6 & 14610.7 & 567.0 & 1119.5 & 26.4 & 474072 & 64.037 & 32.674 & 0.02 \\
\hline TRBY-32_7-B 2 & 316.2 & 1425.0 & 11871.7 & 866.2 & 158.7 & 131.8 & 482608 & 65.190 & 32.945 & 0.83 \\
\hline TRBY -32F_1-B 1 & 1273.4 & 753.5 & 16416.9 & 2078.8 & 722.8 & 448.2 & 485962 & 65.643 & 33.721 & 0.62 \\
\hline TRBY -32F_1-N & 368.8 & 565.1 & 17019.0 & 614.2 & 211.6 & 96.7 & 484785 & 65.484 & 33.167 & 0.46 \\
\hline TRBY-32F_1-B 2 & 289.8 & 1130.2 & 15136.4 & 976.4 & 132.2 & 105.5 & 488656 & 66.007 & 33.206 & 0.80 \\
\hline TRBY-32F_2-N & 509.4 & 1097.4 & 12821.5 & 3291.5 & 299.7 & 219.7 & 480047 & 64.844 & 32.678 & 0.73 \\
\hline TRBY -32F_2-B & 105.4 & 1269.4 & 12007.4 & 606.3 & 52.9 & 8.8 & 477359 & 64.481 & 32.833 & 0.17 \\
\hline TRBY-32F_3-N & 360.1 & 417.7 & 11464.7 & 2763.9 & 6999.1 & 8524.5 & 468712 & 63.313 & 32.620 & 1.22 \\
\hline TRBY-32F_3-B & 289.8 & 1105.6 & 14848.1 & 858.3 & 528.9 & 184.6 & 483748 & 65.344 & 32.713 & 0.35 \\
\hline TRBY-32F_4-N & 579.6 & 1564.2 & 11795.4 & 2952.9 & 1463.3 & 957.9 & 469719 & 63.449 & 32.259 & 0.65 \\
\hline TRBY-32F_4-B & 386.4 & 1040.1 & 13092.8 & 2614.3 & 246.8 & 210.9 & 474050 & 64.034 & 32.788 & 0.85 \\
\hline TRBY-32F_5-N & 412.8 & 1171.1 & 13516.8 & 1677.2 & 299.7 & 158.2 & 481350 & 65.020 & 33.293 & 0.53 \\
\hline TRBY-32F_5-B & 193.2 & 1294.0 & 12397.5 & 622.1 & 88.2 & 70.3 & 482327 & 65.152 & 33.484 & 0.80 \\
\hline TRBY-32F_6-B & 1036.3 & 941.8 & 12389.0 & 5504.2 & 1419.2 & 1168.8 & 472310 & 63.799 & 32.842 & 0.82 \\
\hline TRBY-32F_6-N & 79.0 & 1113.8 & 17281.8 & 559.1 & 211.6 & 175.8 & 481979 & 65.105 & 33.374 & 0.83 \\
\hline TRBY-32F_7-N 1 & 193.2 & 655.2 & 11914.1 & 448.8 & 185.1 & 79.1 & 482823 & 65.219 & 33.099 & 0.43 \\
\hline TRBY-32F_7-N 2 & 649.9 & 1081.0 & 12439.9 & 2378.1 & 493.6 & 290.0 & 475131 & 64.180 & 32.596 & 0.59 \\
\hline TRBY-32F_7-B & 149.3 & 753.5 & 14296.9 & 1401.6 & 669.9 & 360.3 & 469505 & 63.420 & 32.812 & 0.54 \\
\hline TRBY-32G_1-B & 193.2 & 1105.6 & 10760.9 & 763.8 & 44.1 & 43.9 & 484881 & 65.497 & 33.214 & 1.00 \\
\hline TRBY-32G_1-N & 158.1 & 1236.6 & 11083.1 & 708.7 & 79.3 & 35.2 & 484244 & 65.411 & 33.542 & 0.44 \\
\hline TRBY-32G_2-B & 307.4 & 1056.5 & 13092.8 & 1567.0 & 52.9 & 61.5 & 485688 & 65.606 & 33.317 & 1.16 \\
\hline TRBY-32G_3-N & 140.5 & 1015.5 & 10913.5 & 1196.9 & 176.3 & 105.5 & 479136 & 64.721 & 32.878 & 0.60 \\
\hline TRBY-32G_4-B 1 & 202.0 & 892.7 & 11396.9 & 881.9 & 26.4 & 26.4 & 475968 & 64.293 & 32.234 & 1.00 \\
\hline TRBY-32G_4-N & 228.3 & 1244.8 & 9421.1 & 1842.6 & 35.3 & 43.9 & 482860 & 65.224 & 33.268 & 1.25 \\
\hline TRBY-32G_4-B 2 & 281.0 & 1015.5 & 14059.5 & 811.1 & 185.1 & 79.1 & 483963 & 65.373 & 33.122 & 0.43 \\
\hline TRBY-32G_5-B & 219.5 & 696.1 & 13364.2 & 527.6 & 97.0 & 43.9 & 456379 & 61.647 & 31.748 & 0.45 \\
\hline TRBY-32G_5-N & 377.6 & 1064.7 & 11897.2 & 1614.3 & 70.5 & 87.9 & 482934 & 65.234 & 33.414 & 1.25 \\
\hline TRBY-32G_6-B & 8.8 & 933.6 & 13457.4 & 543.3 & 44.1 & 8.8 & 481505 & 65.041 & 33.293 & 0.20 \\
\hline TRBY-32G_6-N & 114.2 & 1474.1 & 11837.8 & 244.1 & 61.7 & 26.4 & 479343 & 64.749 & 32.433 & 0.43 \\
\hline TRBY-32G_7-N & 210.8 & 1408.6 & 11608.8 & 590.6 & 79.3 & 17.6 & 482704 & 65.203 & 32.636 & 0.22 \\
\hline TRBY-32G_8-B 1 & 175.6 & 712.5 & 13355.7 & 818.9 & 229.2 & 70.3 & 481653 & 65.061 & 33.154 & 0.31 \\
\hline TRBY-32G_8-N & 509.4 & 827.2 & 11880.2 & 2354.4 & 167.5 & 193.3 & 474531 & 64.099 & 32.690 & 1.15 \\
\hline TRBY-32G_8-B 2 & 149.3 & 819.0 & 13364.2 & 393.7 & 61.7 & 35.2 & 481224 & 65.003 & 32.989 & 0.57 \\
\hline TRBY-32G_9-N & 351.3 & 286.6 & 9989.2 & 1141.8 & 79.3 & 35.2 & 473754 & 63.994 & 32.207 & 0.44 \\
\hline TRBY-32G_9-B & 166.9 & 655.2 & 13237.0 & 433.1 & 70.5 & 35.2 & 473946 & 64.020 & 32.302 & 0.50 \\
\hline TRBY-32G_10-B & 149.3 & 982.8 & 12863.9 & 1078.8 & 158.7 & 35.2 & 477396 & 64.486 & 32.906 & 0.22 \\
\hline TRBY-32G_11N 2 & 324.9 & 1154.7 & 13406.6 & 1078.8 & 79.3 & 52.7 & 477485 & 64.498 & 32.368 & 0.66 \\
\hline Mean & 327.6 & 920.9 & 12408.5 & 1537.8 & 431.3 & 361.4 & 477326 & 64.5 & 32.8 & 0.8 \\
\hline
\end{tabular}

IV Table 02 - Chemical mineral analyses of zircon grains from Nhandu Intrusive Suite at the Novo Mundo region (MT). 


\begin{tabular}{|c|c|c|c|c|c|c|c|c|c|c|}
\hline Sample & $\begin{array}{c}\mathrm{Yb} \\
\mathrm{ppm}\end{array}$ & $\begin{array}{c}\mathrm{Ta} \\
\mathrm{ppm}\end{array}$ & $\begin{array}{c}\text { Hf } \\
\text { ppm }\end{array}$ & $\begin{array}{c}\mathrm{Y} \\
\mathrm{ppm}\end{array}$ & $\begin{array}{c}\mathrm{U} \\
\mathrm{ppm}\end{array}$ & $\begin{array}{c}\text { Th } \\
\text { ppm }\end{array}$ & $\begin{array}{c}\mathrm{Zr} \\
\mathrm{ppm}\end{array}$ & $\begin{array}{c}\mathrm{ZrO}_{2} \\
\%\end{array}$ & $\begin{array}{l}\mathrm{SiO}_{2} \\
\% \\
\end{array}$ & Th/U \\
\hline TRBY-25_1-B & 878.2 & 1171.1 & 17146.16 & 645.7 & 299.7 & 131.8 & 490559 & 66.264 & 32.3460 & 0.44 \\
\hline TRBY-25_1-N & 61.5 & 884.5 & 8081.25 & 716.6 & 114.6 & 96.7 & 505151 & 68.235 & 32.8090 & 0.84 \\
\hline TRBY-25_2-N & 166.9 & 1375.9 & 10209.68 & 1354.4 & 105.8 & 79.1 & 490330 & 66.233 & 32.5040 & 0.75 \\
\hline TRBY-25_3-N & 316.2 & 1318.5 & 9005.55 & 1748.1 & 35.3 & 184.6 & 501145 & 67.694 & 32.8750 & 5.23 \\
\hline TRBY-25_3-B & 263.5 & 778.0 & 9395.62 & 2126.1 & 61.7 & 281.2 & 495312 & 66.906 & 32.1360 & 4.56 \\
\hline TRBY-25_4-B & 1940.8 & 1302.2 & 16052.26 & 6937.3 & 1428.0 & 290.0 & 443016 & 59.842 & 27.0910 & 0.20 \\
\hline TRBY-25_5-N & 544.5 & 475.0 & 9454.98 & 2330.8 & 282.1 & 325.2 & 497370 & 67.184 & 32.1820 & 1.15 \\
\hline TRBY-25_5-B & 307.4 & 597.8 & 10548.87 & 716.6 & 158.7 & 175.8 & 493565 & 66.67 & 31.8920 & 1.11 \\
\hline TRBY-25_6-N & 254.7 & 1179.3 & 10972.86 & 480.3 & 105.8 & 52.7 & 497784 & 67.24 & 32.2750 & 0.50 \\
\hline TRBY-25_7-B & 263.5 & 1089.2 & 10998.30 & 3212.8 & 26.4 & 61.5 & 499213 & 67.433 & 31.6210 & 2.33 \\
\hline TRBY-25_8-N 1 & 272.2 & 868.1 & 9353.22 & 2441.1 & 44.1 & 105.5 & 494024 & 66.732 & 32.1110 & 2.39 \\
\hline TRBY-25_8-B & 26.3 & 1244.8 & 11295.09 & 464.6 & 264.5 & 114.2 & 498303 & 67.31 & 32.6560 & 0.43 \\
\hline TRBY-25_9-N & 421.5 & 1056.5 & 12796.02 & 1496.1 & 555.3 & 694.3 & 489278 & 66.091 & 31.7560 & 1.25 \\
\hline TRBY-25_9-B & 61.5 & 966.4 & 9929.85 & 1015.8 & 149.9 & 131.8 & 497784 & 67.24 & 32.2390 & 0.88 \\
\hline TRBY-25_10-N & 289.8 & 1351.3 & 9081.87 & 1834.7 & 35.3 & 158.2 & 496171 & 67.022 & 31.5930 & 4.49 \\
\hline TRBY-20_10-N & 289.8 & 720.7 & 9090.35 & 1354.4 & 132.2 & 61.5 & 485147 & 65.533 & 32.7290 & 0.47 \\
\hline TRBY-20_10-B & 140.5 & 892.7 & 9319.30 & 614.2 & 149.9 & 8.8 & 486917 & 65.772 & 33.1880 & 0.06 \\
\hline TRBY-20_9-B 1 & 412.8 & 638.8 & 8776.59 & 2433.2 & 308.5 & 140.6 & 487909 & 65.906 & 33.1790 & 0.46 \\
\hline TRBY-20_8-B & 96.6 & 556.9 & 10252.08 & 677.2 & 114.6 & 87.9 & 486006 & 65.649 & 33.0170 & 0.77 \\
\hline TRBY-20_7-N & 158.1 & 581.5 & 9726.33 & 1732.4 & 255.6 & 158.2 & 475819 & 64.273 & 32.4900 & 0.62 \\
\hline TRBY-20_7-B & 202.0 & 884.5 & 10057.04 & 1472.5 & 158.7 & 43.9 & 483430 & 65.301 & 33.0430 & 0.28 \\
\hline TRBY-20_6-B 1 & 114.2 & 884.5 & 10201.20 & 1031.5 & 176.3 & 87.9 & 478995 & 64.702 & 33.1260 & 0.50 \\
\hline TRBY-20_6-B 2 & 35.1 & 974.6 & 9404.10 & 685.1 & 185.1 & 96.7 & 486243 & 65.681 & 32.9660 & 0.52 \\
\hline TRBY-20_6-N & 649.9 & 728.9 & 8751.15 & 2071.0 & 326.2 & 237.3 & 481483 & 65.038 & 32.4370 & 0.73 \\
\hline TRBY-20_4-N & 228.3 & 1277.6 & 9158.18 & 1196.9 & 167.5 & 114.2 & 482593 & 65.188 & 32.5520 & 0.68 \\
\hline TRBY-20_5-N & 26.3 & 1048.3 & 9132.74 & 929.2 & 26.4 & 61.5 & 481868 & 65.09 & 32.8170 & 2.33 \\
\hline TRBY-20_5-B & 131.7 & 982.8 & 10379.28 & 551.2 & 52.9 & 26.4 & 483696 & 65.337 & 32.8150 & 0.50 \\
\hline TRBY-20_3-N & 175.6 & 974.6 & 9310.82 & 1456.8 & 238.0 & 149.4 & 478055 & 64.575 & 32.4070 & 0.63 \\
\hline TRBY-20_3-B & 210.8 & 1064.7 & 11948.04 & 921.3 & 238.0 & 114.2 & 481461 & 65.035 & 32.9770 & 0.48 \\
\hline TRBY-20_2-B & 17.6 & 925.4 & 9175.14 & 567.0 & 44.1 & 26.4 & 482808 & 65.217 & 33.0890 & 0.60 \\
\hline TRBY-20_2-N & 281.0 & 991.0 & 8963.15 & 1811.1 & 167.5 & 87.9 & 478655 & 64.656 & 33.0510 & 0.52 \\
\hline TRBY-20_1-N & 245.9 & 1056.5 & 10955.90 & 1055.2 & 361.4 & 184.6 & 483408 & 65.298 & 33.1680 & 0.51 \\
\hline TRBY-20_1-B & 289.8 & 835.3 & 10548.87 & 2338.7 & 317.3 & 167.0 & 477182 & 64.457 & 32.8710 & 0.53 \\
\hline TRBY-20_10-N & 447.9 & 655.2 & 11956.52 & 1606.4 & 537.7 & 307.6 & 480106 & 64.852 & 32.7590 & 0.57 \\
\hline TRBY-20_10-B & 193.2 & 647.0 & 14788.77 & 567.0 & 343.8 & 87.9 & 480098 & 64.851 & 32.6880 & 0.26 \\
\hline TRBY-20_11-N & 202.0 & 1031.9 & 8869.87 & 1842.6 & 220.4 & 96.7 & 479788 & 64.809 & 33.2380 & 0.44 \\
\hline TRBY-20_11-B & 140.5 & 679.7 & 11261.17 & 968.6 & 264.5 & 123.0 & 479366 & 64.752 & 32.5560 & 0.47 \\
\hline Mean & 290.8 & 937.6 & 10441.8 & 1497.4 & 228.5 & 144.6 & 486217 & 65.7 & 32.5 & 1.1 \\
\hline
\end{tabular}

\section{Table 03 - Chemical mineral analyses of zircon grains from Matupá Intrusive Suite at the}

\section{Novo Mundo region (MT).}




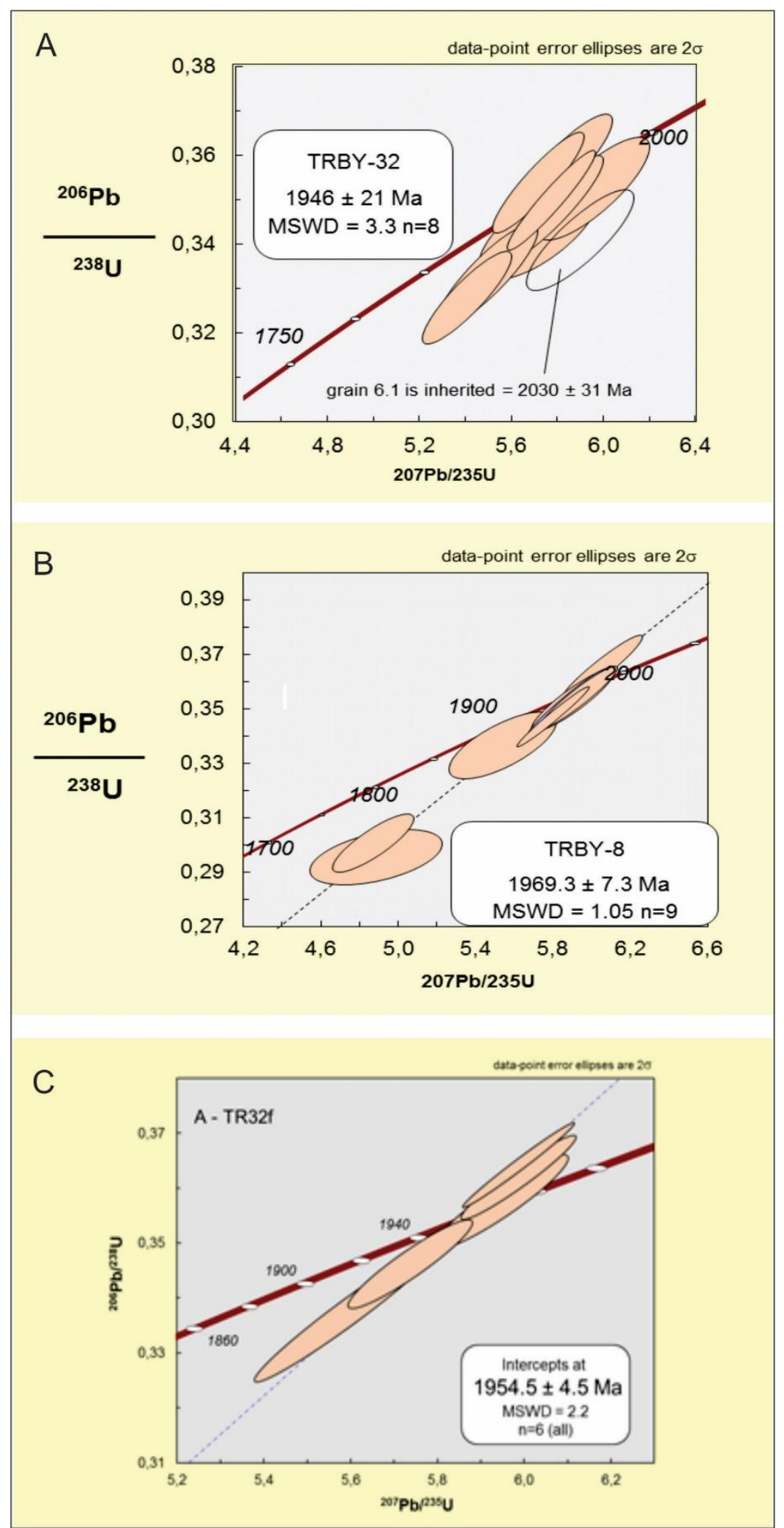

IV Figure 09 -Concordia diagram U/Pb (SHRIMP) for Nhandu Intrusive Suite, (A) sample TRBY -32, showing a crystallization age of $1946 \pm 21 \mathrm{Ma}$; (B) Concordia diagram U/Pb (SHRIMP) from sample TRBY -08, revealing a concordante age by the superior intercept of 
$1969 \pm 7.3 \mathrm{Ma} ;(\mathrm{C})$ Concordia diagram U / Pb (SHRIMP) sample TRBY $-32 F, 1954.5 \pm 4.5$ Ma.

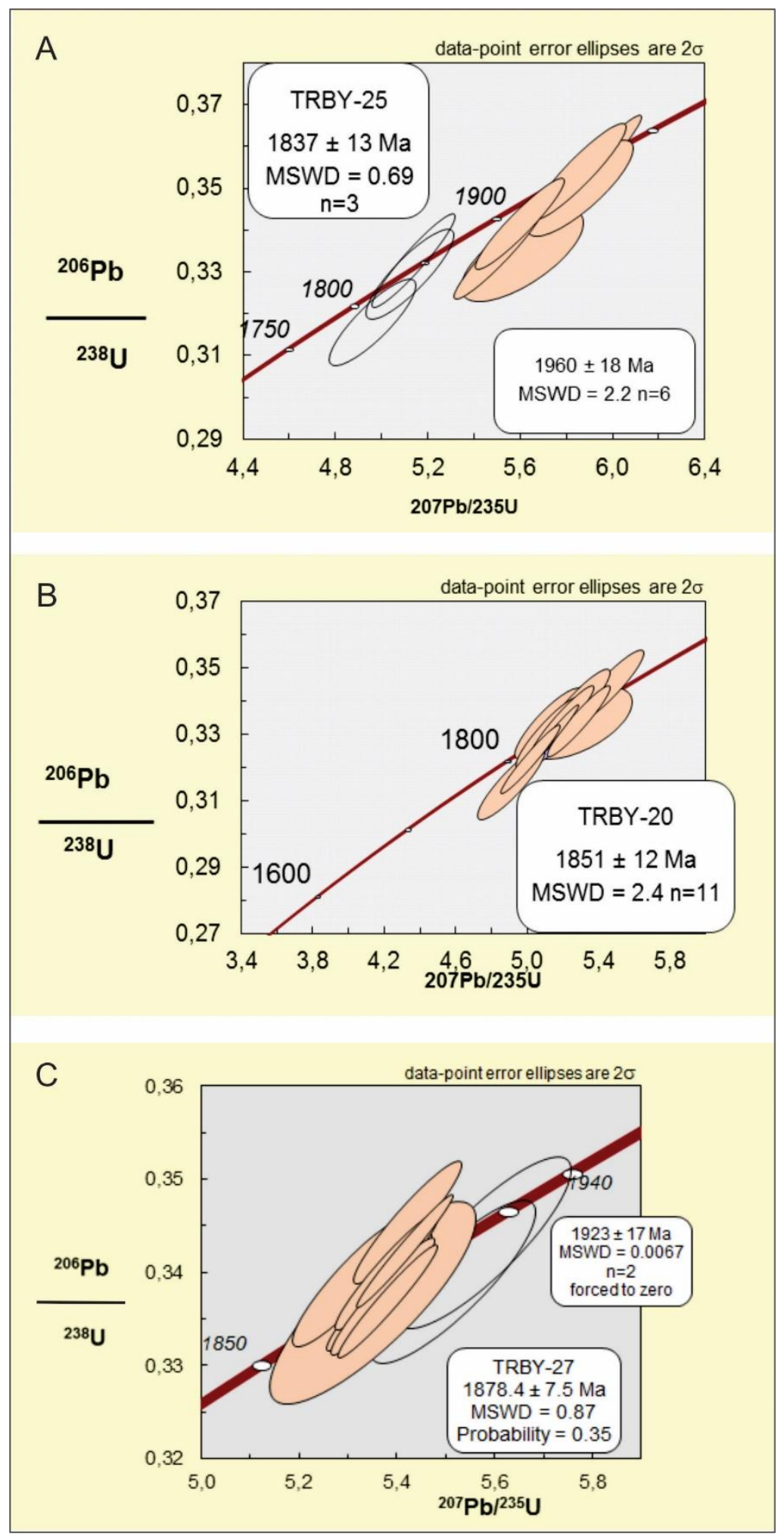

IV Figure 10 -Concordia diagram U/Pb (SHRIMP) for Matupá Intrusive Suite, (A) sample TRBY-25, crystallization age of $1837 \pm 13 \mathrm{Ma}$ and heritage with age $1960 \pm 18 \mathrm{Ma}$; (B) Concordia diagram U/Pb (SHRIMP) of TRBY -20 sample showing crystallization age of 1851 
$\pm 12 \mathrm{Ma}$; (C) Concordia diagram U/Pb (SHRIMP) sample TRBY-27 crystallization age of $1878.4 \pm 7.5 \mathrm{Ma}$ and heritage with age $1923 \pm 17 \mathrm{Ma}$.

\begin{tabular}{|c|c|c|c|c|c|c|c|c|c|c|}
\hline $\begin{array}{l}\text { SAMPLE } \\
\text { TRBY } 32\end{array}$ & $\begin{array}{r}\mathrm{ppm} \\
\mathrm{U} \\
\end{array}$ & $\begin{array}{r}\mathrm{ppm} \\
\mathrm{Th}\end{array}$ & $\begin{array}{l}{ }^{232} \mathrm{Th} \\
\rho^{238} \mathrm{U}\end{array}$ & $\begin{array}{r}204 \text { corr } \\
{ }^{206} \mathrm{~Pb} /{ }^{238} \\
\mathrm{U} \\
\mathrm{Age} \\
\end{array}$ & $\begin{array}{r}1 \mathrm{~s} \\
\text { err }\end{array}$ & $\begin{array}{r}204 \text { corr } \\
{ }^{207} \mathrm{~Pb} /{ }^{206} \mathrm{~Pb} \\
\mathrm{Age} \\
\end{array}$ & $\begin{array}{l}1 \mathrm{~s} \\
\text { err }\end{array}$ & $\begin{array}{r}204 \text { corr } \\
{ }^{208} \mathrm{~Pb} /{ }^{232} \mathrm{Th} \\
\text { Age }\end{array}$ & $\begin{array}{r}1 \mathrm{~s} \\
\mathrm{err}\end{array}$ & $\begin{array}{r}\% \\
\text { Dis- } \\
\text { cor- } \\
\text { dant }\end{array}$ \\
\hline $32 \mathrm{a}$ & 173 & 127 & 0.76 & 1619.1 & 23.6 & 1900 & 27 & 1538 & 43 & 17 \\
\hline $32 \mathrm{a}-11.1$ & 189 & 129 & 0.70 & 1870.4 & 26.6 & 1945 & 20 & 1719 & 44 & 4 \\
\hline $32 a-8.2$ & 139 & 85 & 0.63 & 1967.7 & 28.0 & 1965 & 18 & 1849 & 48 & 0 \\
\hline $32 \mathrm{a}-11.2$ & 124 & 50 & 0.41 & 1715.7 & 25.5 & 1969 & 47 & 1710 & 121 & 15 \\
\hline $32 \mathrm{a}-12.1$ & 79 & 77 & 1.00 & 1793.1 & 27.8 & 2102 & 22 & 1869 & 46 & 17 \\
\hline $32 \mathrm{a}-13.1$ & 50 & 37 & 0.77 & 1735.6 & 29.3 & 1796 & 74 & 1539 & 91 & 4 \\
\hline $32 \mathrm{a}-14.1$ & 152 & 87 & 0.59 & 1872.3 & 26.8 & 1856 & 23 & 1757 & 54 & -1 \\
\hline 32.15 .1 & 81 & 88 & 1.12 & 1867.4 & 28.7 & 1965 & 26 & 1864 & 47 & 5 \\
\hline 32.16 .1 & 180 & 136 & 0.78 & 1893.6 & 26.8 & 1971 & 16 & 1812 & 38 & 4 \\
\hline 32.16 .2 & 132 & 90 & 0.71 & 1235.6 & 23.5 & 2060 & 117 & 1602 & 141 & 67 \\
\hline 32.18 .1 & 231 & 240 & 1.08 & 1975.6 & 27.7 & 1946 & 15 & 1914 & 50 & -1 \\
\hline TRBY 08 & $\begin{array}{r}\mathrm{ppm} \\
\mathrm{U}\end{array}$ & $\begin{array}{r}\mathrm{ppm} \\
\mathrm{Th}\end{array}$ & $\begin{array}{c}{ }^{232} \mathrm{Th} \\
{ }^{238} \mathrm{U}\end{array}$ & $\begin{array}{r}204 \text { corr } \\
{ }^{206} \mathrm{~Pb} /{ }^{238} \\
\mathrm{U} \\
\text { Age }\end{array}$ & $\begin{array}{l}1 \mathrm{~s} \\
\text { err }\end{array}$ & $\begin{array}{r}204 \text { corr } \\
{ }^{207} \mathrm{~Pb} /{ }^{206} \mathrm{~Pb} \\
\mathrm{Age} \\
\end{array}$ & $\begin{array}{l}1 \mathrm{~s} \\
\text { err }\end{array}$ & $\begin{array}{r}204 \text { corr } \\
{ }^{208} \mathrm{~Pb} /{ }^{232} \mathrm{Th} \\
\text { Age }\end{array}$ & $\begin{array}{r}1 \mathrm{~s} \\
\mathrm{err}\end{array}$ & $\begin{array}{r}\% \\
\text { Dis- } \\
\text { cor- } \\
\text { dant }\end{array}$ \\
\hline $08-7.2$ & 388 & 353 & 0.94 & 1866.8 & 25.9 & 1972 & 37 & 1559 & 45 & 6 \\
\hline $08-5.2$ & 380 & 235 & 0.64 & 1719.7 & 25.3 & 2016 & 41 & 1533 & 64 & 17 \\
\hline $08-8.1$ & 86 & 118 & 1.42 & 1545.0 & 27.8 & 1880 & 131 & 837 & 79 & 22 \\
\hline $08-9.1$ & 84 & 88 & 1.09 & 1927.0 & 29.3 & 1917 & 47 & 1748 & 65 & -1 \\
\hline $08-10.1$ & 232 & 117 & 0.52 & 1763.9 & 25.5 & 2004 & 46 & 1586 & 87 & 14 \\
\hline $08-11.1$ & 146 & 159 & 1.12 & 1957.5 & 27.8 & 2047 & 16 & 1893 & 39 & 5 \\
\hline $08-13.1$ & 285 & 140 & 0.51 & 1869.4 & 26.0 & 1927 & 21 & 1718 & 41 & 3 \\
\hline $08-14.1$ & 131 & 97 & 0.77 & 1863.8 & 26.9 & 1854 & 16 & 1758 & 39 & -1 \\
\hline $08-6.2$ & 498 & 343 & 0.71 & 1338.5 & 19.2 & 1756 & 14 & 1048 & 22 & 31 \\
\hline TRBY 32F & $\begin{array}{r}\mathrm{ppm} \\
\mathrm{U}\end{array}$ & $\begin{array}{r}\mathrm{ppm} \\
\mathrm{Th}\end{array}$ & $\begin{array}{c}{ }^{232} \mathrm{Th} \\
\rho^{238} \mathrm{U}\end{array}$ & $\begin{array}{r}204 \text { corr } \\
{ }^{206} \mathrm{~Pb} /{ }^{238} \\
\mathrm{U} \\
\text { Age }\end{array}$ & $\begin{array}{l}1 \mathrm{~s} \\
\text { err }\end{array}$ & $\begin{array}{r}204 \text { corr } \\
{ }^{207} \mathrm{~Pb} /{ }^{206} \mathrm{~Pb} \\
\mathrm{Age}\end{array}$ & $\begin{array}{l}1 \mathrm{~s} \\
\text { err }\end{array}$ & $\begin{array}{r}204 \text { corr } \\
{ }^{208} \mathrm{~Pb} /{ }^{232} \mathrm{Th} \\
\text { Age }\end{array}$ & $\begin{array}{r}1 \mathrm{~s} \\
\mathrm{err}\end{array}$ & $\begin{array}{r}\% \\
\text { Dis- } \\
\text { cor- } \\
\text { dant }\end{array}$ \\
\hline 1505A.3-1 & 1322 & 79 & 0.06 & 2003 & 30 & 1944 & 5 & 2103 & 77 & -4 \\
\hline 1505A.3-2 & 192 & 235 & 1.26 & 1971 & 33 & 1966 & 14 & 1940 & 40 & -0 \\
\hline 1505A.4-1 & 1513 & 840 & 0.57 & 1992 & 30 & 1955 & 9 & 1964 & 33 & -2 \\
\hline 1505A.5-1 & 285 & 66 & 0.24 & 1875 & 50 & 1960 & 13 & 1980 & 93 & 5 \\
\hline 1505A.56-1 & 278 & 167 & 0.62 & 1917 & 32 & 1959 & 13 & 1883 & 39 & 2 \\
\hline
\end{tabular}

IV Table 04- SHRIMP U-Pb zircon data of Nhandu granitic rocks. 


\begin{tabular}{|c|c|c|c|c|c|c|c|c|c|c|}
\hline $\begin{array}{l}\text { SAMPLE } \\
\text { TRBY } 25\end{array}$ & $\begin{array}{r}\text { ppm } \\
\mathrm{U}\end{array}$ & $\begin{array}{r}\text { ppm } \\
\text { Th }\end{array}$ & $\begin{array}{l}{ }^{232} \mathrm{Th} \\
{ }^{238} \mathrm{U}\end{array}$ & $\begin{array}{r}204 \text { corr } \\
{ }^{206} \mathrm{~Pb} /{ }^{238} \mathrm{U} \\
\text { Age }\end{array}$ & $\begin{array}{l}1 \mathrm{~s} \\
\text { err }\end{array}$ & $\begin{array}{r}204 \text { corr } \\
{ }^{207} \mathrm{~Pb} /{ }^{206} \mathrm{~Pb} \\
\mathrm{Age} \\
\end{array}$ & $\begin{array}{l}1 \mathrm{~s} \\
\text { err }\end{array}$ & $\begin{array}{r}204 \text { corr } \\
{ }^{208} \mathrm{~Pb} /{ }^{232} \mathrm{Th} \\
\text { Age }\end{array}$ & $\begin{array}{l}1 \mathrm{~s} \\
\text { err }\end{array}$ & $\begin{array}{r}\% \\
\text { Dis- } \\
\text { cor- } \\
\text { dant }\end{array}$ \\
\hline $25-2.1$ & 300 & 239 & 0.82 & 1894,3 & 42.6 & 1938 & 17 & 1838 & 52 & 2 \\
\hline $25-4.1$ & 429 & 562 & 1.35 & 1862,5 & 44.1 & 1944 & 17 & 1773 & 50 & 4 \\
\hline $25-5.1$ & 274 & 295 & 1.11 & 1852,6 & 43.7 & 1831 & 17 & 1709 & 49 & -1 \\
\hline $25-7.1$ & 215 & 221 & 1.06 & 1781,3 & 40.7 & 1848 & 29 & 1619 & 49 & 4 \\
\hline $25-8.1$ & 110 & 115 & 1.08 & 1934,8 & 44.3 & 1976 & 32 & 1829 & 59 & 2 \\
\hline $25-9.1$ & 155 & 107 & 0.71 & 1837,1 & 42.2 & 1843 & 28 & 1676 & 64 & 0 \\
\hline $25-10.1$ & 96 & 96 & 1.03 & 1858,7 & 43.2 & 1981 & 49 & 1760 & 73 & 7 \\
\hline TRBY 20 & $\begin{array}{r}\mathrm{ppm} \\
\mathrm{U}\end{array}$ & $\begin{array}{r}\text { ppm } \\
\text { Th }\end{array}$ & $\begin{array}{l}{ }^{232} \mathrm{Th} \\
\rho^{238} \mathrm{U}\end{array}$ & $\begin{array}{r}204 \text { corr } \\
{ }^{206} \mathrm{~Pb} \\
{ }^{238} \mathrm{U} \\
\text { Age }\end{array}$ & $\begin{array}{l}1 \mathrm{~s} \\
\text { err }\end{array}$ & $\begin{array}{r}204 \text { corr } \\
{ }^{207} \mathrm{~Pb} \\
/^{206} \mathrm{~Pb} \\
\text { Age }\end{array}$ & $\begin{array}{l}1 \mathrm{~s} \\
\text { err }\end{array}$ & $\begin{array}{r}204 \text { corr } \\
{ }^{208} \mathrm{~Pb} \\
{ }^{232} \mathrm{Th} \\
\text { Age }\end{array}$ & $\begin{array}{l}1 \mathrm{~s} \\
\text { err }\end{array}$ & $\begin{array}{r}\% \\
\text { Dis- } \\
\text { cor- } \\
\text { dant }\end{array}$ \\
\hline 20.11 .1 & 168 & 86 & 0.53 & 1842,0 & 26.2 & 1844 & 22 & 1754 & 52 & 0 \\
\hline 20.12 .1 & 256 & 120 & 0.48 & 1789,5 & 25.5 & 1854 & 20 & 1745 & 49 & 4 \\
\hline 20.13 .1 & 254 & 143 & 0.58 & 1702,6 & 24.5 & 1875 & 56 & 1727 & 85 & 10 \\
\hline 20.14 .1 & 129 & 66 & 0.53 & 1839,7 & 27.1 & 1874 & 23 & 1696 & 56 & 2 \\
\hline 20.15 .1 & 148 & 68 & 0.48 & 1851,2 & 26.5 & 1840 & 23 & 1761 & 52 & -1 \\
\hline 20.16 .1 & 104 & 57 & 0.56 & 1856,1 & 27.3 & 1855 & 33 & 1702 & 67 & 0 \\
\hline 20.17 .1 & 323 & 184 & 0.59 & 1862,6 & 26.0 & 1865 & 14 & 1778 & 37 & 0 \\
\hline TRBY 27 & $\begin{array}{r}\text { ppm } \\
\mathrm{U}\end{array}$ & $\begin{array}{r}\text { ppm } \\
\text { Th }\end{array}$ & $\begin{array}{l}{ }^{232} \mathrm{Th} \\
{ }^{238} \mathrm{U}\end{array}$ & $\begin{array}{r}{ }^{204} \text { corr } \\
{ }^{206} \mathrm{~Pb} \\
{ }^{238} \mathrm{U} \\
\text { Age }\end{array}$ & $\begin{array}{l}1 \mathrm{~s} \\
\text { err }\end{array}$ & $\begin{array}{r}204 \text { corr } \\
{ }^{207} \mathrm{~Pb} \\
2^{206} \mathrm{~Pb} \\
\text { Age }\end{array}$ & $\begin{array}{l}1 \mathrm{~s} \\
\text { err }\end{array}$ & $\begin{array}{r}204 \text { corr } \\
{ }^{208} \mathrm{~Pb} \\
{ }^{232} \mathrm{Th} \\
\text { Age }\end{array}$ & $\begin{array}{l}1 \mathrm{~s} \\
\mathrm{err}\end{array}$ & $\begin{array}{r}\% \\
\text { Dis- } \\
\text { cor- } \\
\text { dant }\end{array}$ \\
\hline 1505G10-1 & 663 & 416 & 0.65 & 1872 & 29 & 1880 & 8 & 1855 & 56 & 0,5 \\
\hline 1505G.4-1 & 1191 & 117 & 0.10 & 1874 & 24 & 1891 & 7 & 1933 & 46 & 1,1 \\
\hline 1505G.4-2 & 74 & 83 & 1.15 & 1904 & 36 & 1922 & 24 & 1918 & 49 & 1,1 \\
\hline 1505G.7-1 & 579 & 402 & 0.72 & 1883 & 29 & 1873 & 9 & 1831 & 34 & $-0,6$ \\
\hline 1505G.1-1 & 82 & 90 & 1.14 & 1884 & 35 & 1924 & 23 & 1910 & 47 & 2,4 \\
\hline 1505G.7-2 & 37 & 41 & 1.13 & 1873 & 43 & 1881 & 32 & 1883 & 60 & 0,5 \\
\hline 1505G.6-1 & 1455 & 609 & 0.43 & 1894 & 28 & 1873 & 5 & 1854 & 32 & $-1,3$ \\
\hline 1505G.9-1 & 562 & 412 & 0.76 & 1876 & 25 & 1885 & 9 & 1827 & 30 & 0,5 \\
\hline 1505G.1-2 & 442 & 333 & 0.78 & 1915 & 26 & 1859 & 10 & 1880 & 31 & $-3,5$ \\
\hline
\end{tabular}

IV Table 05- SHRIMP U-Pb zircon data of Matupá granitic rocks. 


\begin{tabular}{|c|c|c|c|c|c|c|c|c|c|c|c|}
\hline \multirow[t]{2}{*}{ Spot } & \multirow{2}{*}{$\begin{array}{l}\mathrm{U} / \mathrm{Pb} \\
\text { Age } \\
\text { (Ma) }\end{array}$} & \multirow[b]{2}{*}{ $\pm 2 \mathrm{~s}$} & \multicolumn{4}{|c|}{ Sample (Present day ratios) } & \multicolumn{4}{|c|}{$\begin{array}{c}\text { Sample Initial } \\
\text { Ratios }\end{array}$} & \multirow{2}{*}{$\begin{array}{r}\mathrm{T}_{\mathrm{DM}} \\
(\mathrm{Ga}) \\
\end{array}$} \\
\hline & & & ${ }^{176} \mathrm{Hf} /{ }^{177} \mathrm{Hf}$ & $\pm 2 \mathrm{SE}$ & ${ }^{176} \mathrm{Lu} /{ }^{177} \mathrm{Hf}$ & $\pm 2 \mathrm{SE}$ & ${ }^{176} \mathrm{Hf} /{ }^{177} \mathrm{Hf}$ & $\mathrm{eHf}(0)$ & $\mathrm{eHf}(\mathrm{t})$ & $\pm 2 \mathrm{SE}$ & \\
\hline 05 TRBY08_5.1 & 1969 & 10 & 0.281451 & 0.000038 & 0.000773 & 0.000101 & 0.281422 & -47.2 & -3.7 & 0.50 & 2.73 \\
\hline 06 TRBY08_4.1 & 1969 & 10 & 0.281391 & 0.000035 & 0.000716 & 0.000036 & 0.281364 & -49.3 & -5.8 & 0.32 & 2.84 \\
\hline 07 TRBY08_4.2 & 1969 & 10 & 0.281409 & 0.000036 & 0.000655 & 0.000015 & 0.281385 & -48.6 & -5.0 & 0.14 & 2.80 \\
\hline BY08_6.1 & 1969 & 10 & 0.281431 & 0.000054 & 0.000607 & 0.000070 & 0.281408 & -47.9 & -4.2 & 0.51 & 2.76 \\
\hline 09 TRBY08_3.1 & 1969 & 10 & 0.281458 & 0.000065 & 0.001701 & 0.000085 & 0.281394 & -46.9 & -4.7 & 0.26 & 2.78 \\
\hline 10 & 1969 & 10 & 0.281471 & 0.000041 & 0.000824 & 0.000065 & 0.281440 & -46.5 & -3.1 & .26 & 2.69 \\
\hline 11 TRBY08_1.2 & 1969 & 10 & 0.281518 & 0.000061 & 0.002702 & 0.000121 & 0.281417 & -44.8 & -3.9 & 0.20 & 2.74 \\
\hline 12 TRBY08_2.1 & 1969 & 10 & 0.281437 & 0.000059 & 0.001232 & 0.000045 & 0.281391 & -47.7 & -4.8 & 0.20 & 2.79 \\
\hline 05 TRBY32G_1.2 & 1946 & 21 & 0.28 & 0.000027 & 0.000 & 009 & 0.281418 & -47.6 & -4.4 & 0.11 & 2.75 \\
\hline 06 TRBY32G_2.1 & 1946 & 21 & 0.281451 & 0.000053 & 0.00 & 35 & 0.281437 & -47.2 & -3.7 & 0.38 & 2.71 \\
\hline 07 TRBY32 & 1946 & 21 & 0.281446 & 0.000032 & 0.0 & 004 & 0.281422 & -47.4 & -4.3 & 0.07 & 2.74 \\
\hline 08 TRBY32G_7.1 & 1946 & 21 & 0.281412 & 0.000057 & 0.000419 & 0.000007 & 0.281396 & -48.6 & -5.2 & 0.14 & 2.79 \\
\hline 09 TRBY32G_5.1 & 1946 & 21 & 0.281428 & 0.000066 & 0.000583 & 0.000037 & 0.281406 & -48.0 & -4.8 & 0.36 & 2.77 \\
\hline 10 TRBY32G_4.1 & 1946 & 21 & 0.281448 & 0.000044 & 0.000808 & 0.000072 & 0.281418 & -47.3 & -4.4 & 0.44 & 2.75 \\
\hline 11 TRBY32G_9.1 & 2066 & 21 & 0.281468 & 0.000047 & 0.000833 & 0.000031 & 0.281435 & -46.6 & -1.0 & 0.05 & 2.65 \\
\hline 12 TRBY32G_11.1 & 1946 & 21 & 0.281427 & 0.000032 & 0.000425 & 0.000036 & 0.281411 & -48.0 & -4.6 & 0.44 & 2.76 \\
\hline
\end{tabular}

IV Table 06 - Lu-Hf isotopic data of zircons from Nhandu granitic rocks, Novo Mundo, Mato Grosso, Brazil. 


\begin{tabular}{|c|c|c|c|c|c|c|c|c|c|c|c|}
\hline \multirow[t]{2}{*}{ Spot } & \multirow{2}{*}{$\begin{array}{l}\mathrm{U} / \mathrm{Pb} \\
\text { Age } \\
\text { (Ma) }\end{array}$} & \multirow[b]{2}{*}{ $\pm 2 \mathrm{~s}$} & \multicolumn{4}{|c|}{ Sample (Present day ratios) } & \multicolumn{2}{|c|}{$\begin{array}{c}\text { Sample Initial } \\
\text { Ratios }\end{array}$} & \multirow[b]{2}{*}{$\mathrm{eHf}(\mathrm{t})$} & \multirow[b]{2}{*}{ $\pm 2 \mathrm{SE}$} & \multirow{2}{*}{$\begin{array}{l}\mathrm{T}_{\mathrm{DM}} \\
(\mathrm{Ga})\end{array}$} \\
\hline & & & ${ }^{176} \mathrm{Hf} /{ }^{177} \mathrm{Hf}$ & $\pm 2 \mathrm{SE}$ & ${ }^{176} \mathrm{Lu} /{ }^{177} \mathrm{Hf}$ & $\pm 2 \mathrm{SE}$ & ${ }^{176} \mathrm{Hf} /{ }^{177} \mathrm{Hf}$ & $\mathrm{eHf}(0)$ & & & \\
\hline 005 TRBY20_9.2 & 1851 & 12 & 0.281468 & 0.000049 & 0.001129 & 0.000032 & 0.281429 & -46.6 & -6.2 & 0.2 & 2.77 \\
\hline 006 TRBY20_8.2 & 1851 & 12 & 0.281468 & 0.000041 & 0.000490 & 0.000023 & 0.281451 & -46.6 & -5.4 & 0.3 & 2.73 \\
\hline 007 TRBY20_5.1 & 1851 & 12 & 0.281491 & 0.000039 & 0.000376 & 0.000004 & 0.281478 & -45.8 & -4.5 & 0.1 & 2.68 \\
\hline 008 TRBY20_7.1 & 1851 & 12 & 0.281450 & 0.000035 & 0.000766 & 0.000047 & 0.281423 & -47.2 & -6.4 & 0.4 & 2.79 \\
\hline 009 TRBY20_4.2 & 1851 & 12 & 0.281486 & 0.000042 & 0.000694 & 0.000046 & 0.281461 & -46.0 & -5.1 & 0.4 & 2.71 \\
\hline 010 TRBY20_3.1 & 1851 & 12 & 0.281440 & 0.000036 & 0.000573 & 0.000004 & 0.281420 & -47.6 & -6.5 & 0.1 & 2.79 \\
\hline 011 TRBY20_2.1 & 1851 & 12 & 0.281487 & 0.000040 & 0.000604 & 0.000053 & 0.281465 & -45.9 & -4.9 & 0.5 & 2.70 \\
\hline 012 TRBY20_1.1 & 1851 & 12 & 0.281467 & 0.000038 & 0.000739 & 0.000032 & 0.281441 & -46.6 & -5.8 & 0.3 & 2.75 \\
\hline 005 TRBY25 & 1837 & 13 & 0.281395 & 0.000033 & 0.000649 & 0.000078 & 0.281373 & -49.1 & -8.5 & 1.1 & 2.89 \\
\hline 006 TRBY25 & 1837 & 13 & 0.281449 & 0.000053 & 0.001694 & 0.000121 & 0.281390 & -47.2 & -7.9 & 0.6 & 2.86 \\
\hline 007 TRBY25 & 1837 & 13 & 0.281384 & 0.000045 & 0.000647 & 0.000036 & 0.281362 & -49.5 & -8.9 & 0.6 & 2.92 \\
\hline 008 TRBY25 & 1837 & 13 & 0.281393 & 0.000047 & 0.000682 & 0.000101 & 0.281369 & -49.2 & -8.7 & 1.4 & 2.90 \\
\hline 009 TRBY25 & 1837 & 13 & 0.281399 & 0.000053 & 0.000758 & 0.000046 & 0.281372 & -49.0 & -8.5 & 0.6 & 2.89 \\
\hline 010 TRBY25 & 1837 & 13 & 0.281367 & 0.000037 & 0.000601 & 0.000033 & 0.281346 & -50.1 & -9.5 & 0.6 & 2.95 \\
\hline 011 TRBY25 & 1837 & 13 & 0.281390 & 0.000046 & 0.001100 & 0.000119 & 0.281352 & -49.3 & -9.3 & 1.1 & 2.93 \\
\hline 012 TRBY25 & 1944 & 13 & 0.281406 & 0.000052 & 0.001324 & 0.000055 & 0.281357 & -48.8 & -6.6 & 0.3 & 2.87 \\
\hline
\end{tabular}

IV Table 07 - Lu-Hf isotopic data of zircons from Matupá granitic rocks, Novo Mundo, Mato Grosso, Brazil. 


\begin{tabular}{|c|c|c|c|c|c|c|c|c|c|c|c|c|}
\hline \multirow[t]{2}{*}{ Sample } & \multirow{2}{*}{$\begin{array}{c}\mathrm{Sm} \\
(\mathrm{ppm})\end{array}$} & \multirow{2}{*}{$\begin{array}{c}\mathrm{Nd} \\
\text { (ppm) }\end{array}$} & \multirow{2}{*}{$\begin{array}{l}{ }^{147} \mathrm{Sm} / \\
{ }^{144} \mathrm{Nd}\end{array}$} & \multirow{2}{*}{$\begin{array}{l}{ }^{143} \mathrm{Nd} / \\
{ }^{144} \mathrm{Nd}\end{array}$} & \multirow{2}{*}{$\begin{array}{l}\text { Error } \\
\pm 2 \mathrm{SE}\end{array}$} & \multirow{2}{*}{$\begin{array}{c}\varepsilon N d \\
0 \\
\end{array}$} & \multirow{2}{*}{$\begin{array}{c}\varepsilon \mathrm{Nd}(\mathrm{t}) \\
(\mathrm{t})\end{array}$} & \multirow{2}{*}{$\begin{array}{r}\varepsilon \mathrm{Hf} \\
(\mathrm{t})\end{array}$} & \multirow{2}{*}{$\begin{array}{c}\mathrm{T}_{\mathrm{DM}} \mathrm{Nd} \\
\text { (Ga) }\end{array}$} & \multicolumn{3}{|c|}{$\mathrm{T}_{\mathrm{DM}} \mathrm{HfCrystallization} \mathrm{fSm}-\mathrm{Nd}$} \\
\hline & & & & & & & & & & (Ga) & Age $(t)$ & \\
\hline TRBY60C Nhandu & 6.5 & 32.7 & 0.1211 & 0.511658 & 6 & -19.12 & -0.12 & & 2.27 & & $1969 \pm 7.3$ & -0.38 \\
\hline TRBY60A Nhandu & 6.9 & 37.6 & 0.1113 & 0.511482 & 14 & -22.54 & -2.53 & & 2.31 & & $1969 \pm 7.3$ & -0.43 \\
\hline TRBY40A Nhandu & 20.0 & 150.9 & 0.0801 & 0.510807 & 23 & -35.72 & -6.42 & & 2.54 & & $1969 \pm 7.3$ & -0.59 \\
\hline TRBY08 Nhandu & 3.8 & 23.8 & 0.0959 & 0.511291 & 12 & -26.27 & -0.95 & -4.4 & 2.27 & 2.62 & $1969 \pm 7.3$ & -0.51 \\
\hline TRBY32F Nhandu & 8.3 & 50.8 & 0.0992 & 0.511275 & 12 & -26.59 & -2.28 & & 2.36 & & $1954 \pm 4.5$ & -0.50 \\
\hline TRBY20 Matupa & 4.2 & 25.1 & 0.1011 & 0.511304 & 17 & -26.02 & -3.44 & -5.6 & 2.36 & 2.49 & $1851 \pm 12$ & -0.49 \\
\hline TRBY25 Matupa & 12.8 & 81.7 & 0.0949 & 0.511268 & 14 & -26.72 & -2.28 & -8.7 & 2.28 & 2.63 & $1837 \pm 13$ & -0.52 \\
\hline TRBY05 Matupa & 7.6 & 44.0 & 0.104 & 0.511417 & 8 & -23.81 & 0.05 & & 2.25 & & $1851 \pm 12$ & -0.47 \\
\hline
\end{tabular}

Table 08 - WR Sm-Nd isotopes for Nhandu and Matupá Intrusive Suite samples, Novo Mundo, Mato Grosso, Brazil. 


\section{7 - DISCUSSION}

The obtained data in this study show for the Trairão region that the Matupá and Nhandu Intrusive Suites are metaluminous to lightly peraluminous, ferrous, K-rich calc-alkaline granites. The REE patterns are compatible to calc-alkaline rocks with small fractionation of light elements, negative anomaly of $\mathrm{Eu}$, while the trace element patterns normalized to chondrite contain negative anomalies of $\mathrm{Ta}, \mathrm{Nb}, \mathrm{Sr}, \mathrm{Ti}$ and $\mathrm{P}$, compatible to subuducted-related magma origin.

$\mathrm{Th} / \mathrm{U}$ ratios zircons various rocks are generally equal to or greater than 0.5 (Hoskin and Schaltegger, 2003; Xiang et al, 2011), whereas zircon affected by metamorphic events show considerably lower ratios ( 0, 01) (Hidaka et al., 2002; Rubatto, 2002). Th/U ratios of most analyzed zircons show average higher than 0.2, confirming its igneous origin. Moreover, the relationship of $\mathrm{Th} / \mathrm{U}$ was shown to be sensitive to temperature variations (Bolhar et al, 2008; Gagnevin et al, 2010). The decrease in the magma temperature promotes high contents of $U$ in relation to Th content, resulting in minor proportions $\mathrm{Th} / \mathrm{U}$ to zircon crystallized from magma lower temperature. The incorporation of Th and $\mathrm{U}$ zircon can be highly affected by the availability of these metals during the partitioning and zircon crystallization with coexisting minerals. Th-U concentrations zircon be controlled mainly by the presence of monazite (Villaseca et al., 2003), which incorporates Th as a major structural components and may indicate crystallization in equilibrium with monazite (Figure 11A) (Hermann and Rubatto 2003). During magmatic differentiation, plenty of $\mathrm{Hf}$ zircon increases as the $\mathrm{Zr} / \mathrm{Hf}$ ratio tends to decrease (Hoskin et al, 2000; Linnen and Keppler, 2002). Therefore, the relationship of Th/U and $\mathrm{Zr} / \mathrm{Hf}$ can be used as index differentiating for zircon. Th/U versus $\mathrm{Zr} / \mathrm{Hf}$ diagram shows good positive correlation with both declining relationships as progressive magmatic evolution (Figure 11B). U/Yb rattios are used to identify zircons of crustal from oceanic origin, so that these values increase progressively from oceanic gabbros $(0.18)$ to continental granites (1.07) and kimberlites (2.1). Discrimination diagrams proposed by Grimes (2007) based on the U/Yb ratios versus $\mathrm{Y}$ and $\mathrm{Hf}$, are used to distinguish between zircons formed in continental and oceanic crust and the mantle (megacrystals kimberlite). In Figure $11 \mathrm{C}$; D, all zircon crystals of all the samples of Nhandu and Matupá granites plot in the field of continental zircons. 

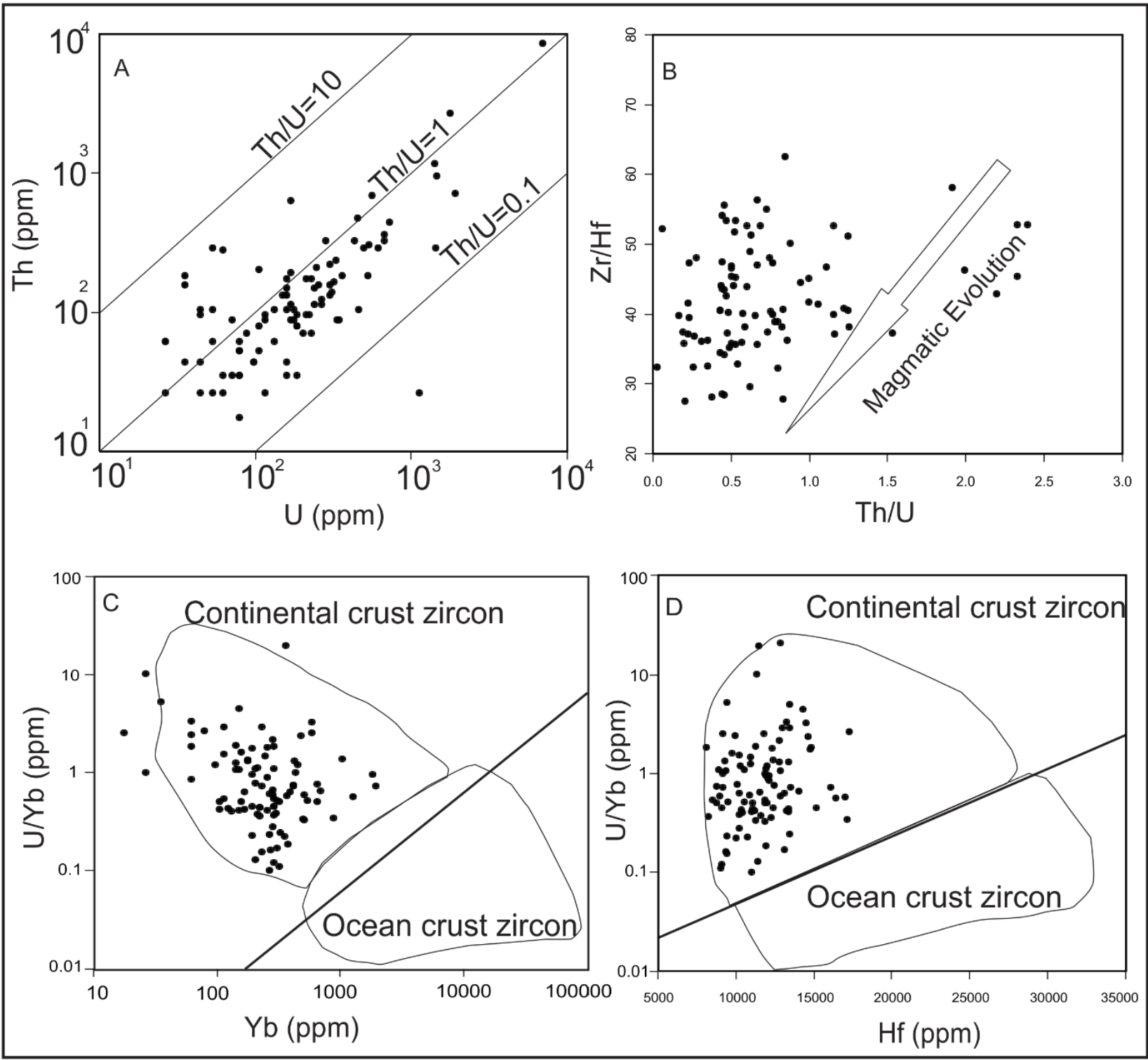

IV Figure 11- A) Variation of Th versus $U$ ratios for zircons from Trairão region; (B) Th/U versus $\mathrm{Zr} / \mathrm{Hf}$ ratios; (C) U/Yb versus $Y$, (D) U/Yb versus $H f$ diagrams with the fields of Grimes et al. (2007) to discriminate between continental and oceanic crust zircon. Heavy lines Indicate the lower limit of zircons from continental crust.

The incorporation of mature continental crust on the source of the granitic bodies of Trairão region is evidenced by the relatively low values of hafnium radiogenic isotopes found in zircons. Therefore, it was observed in this study that is required developing of more detailed studies to answer questions about the petrogenesis of the region. Shaded areas represent a trends of crustal evolution of zircons that can originate from areas of crust (CA - Archean crust and CT - Transamazonic crust). The trend of the average evolution of the Archean upper crust was 
calculated by Condie et al. (2005) assuming a crustal proportion ${ }^{176} \mathrm{Lu} /{ }^{177} \mathrm{Lu}$ of 0.013 . The samples are plotted on $\varepsilon H f$ values (t) versus Hf isotopic evolution diagram (Figure 12).

The melt of the magma may have occurred at higher depths without participation of fluids under high temperatures, low melting metassomatized lithospheric plate. The rise of molten material still warm, lived a certain period in shallower areas and assimilated crustal materials resulting in inheritance oldest zircons and slightly negative Hf signature. This procedure is considered common for granites formed in active continental margin environment and intra-plate (Clemens and Wall, 1984; Clemens and Watkins, 2001 Stevens and Clemens, 1993), where in these conditions is common the generation of granites with high silica content and low content of mafic minerals.

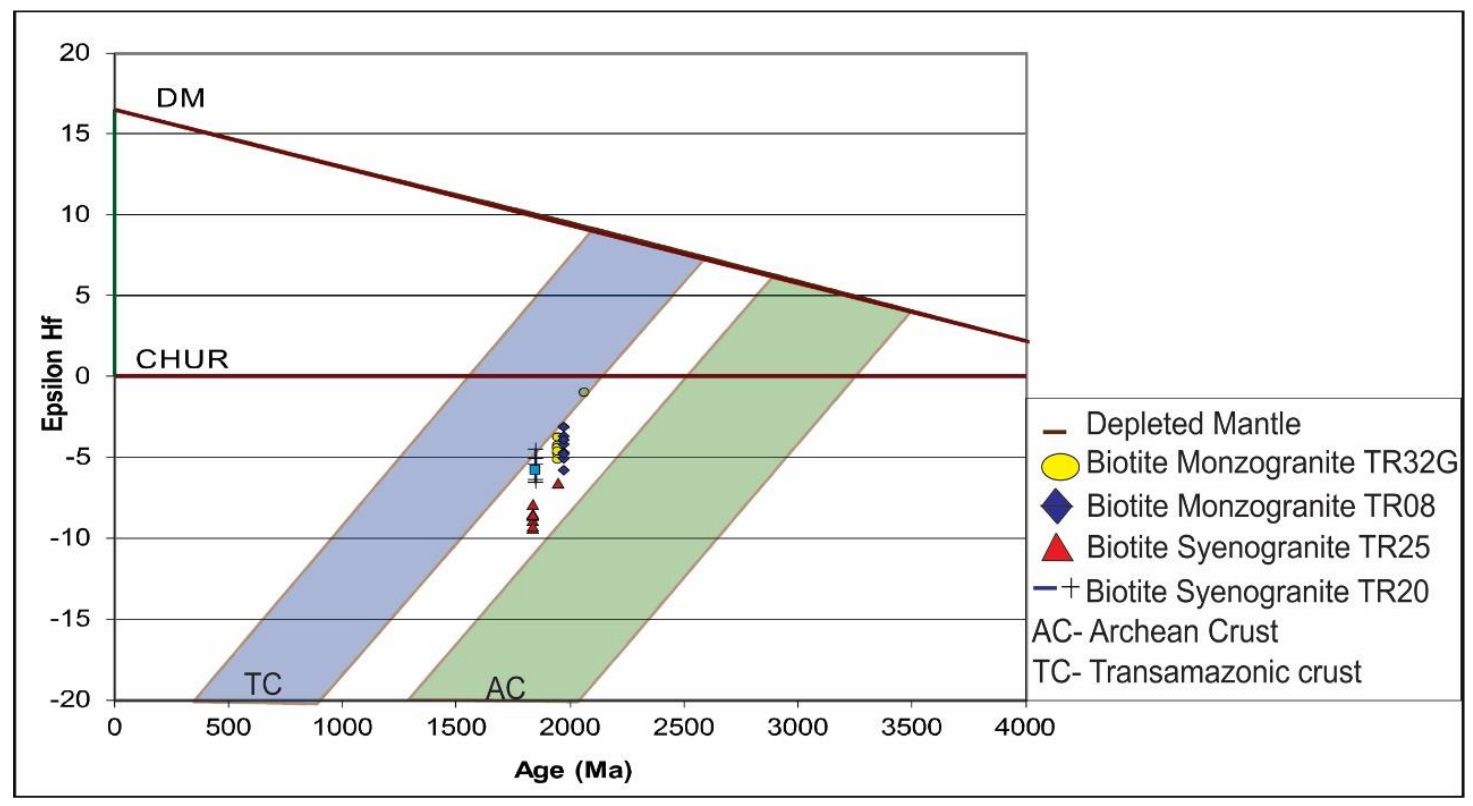

IV Figure 12-Diagram of EHf values ( $t$ ) and Hf isotopic evolution, where blue = Transmazonian crust evolution, green $=$ Archean crust evolution .

Based on SHRIMP U-Pb zircon ages, we characterized two main pulses of magmatism at the Trairão region. The first, those granites correlated to the Nhandu Intrusive Suite, are represented by $1969 \pm$ Ma intrusion (biotite-granite), $1954 \pm 4 \mathrm{Ma}$ (second intrusion of biotite-granite) and $1946 \pm 21 \mathrm{Ma}$ (third intrusion -granite biotite) (Figure 09). Samples of Nhandu Intrusive Suite have $\varepsilon \mathrm{Hf}(\mathrm{t}=1946 \mathrm{Ma})$ values from -3.1 to -5.8 and $\mathrm{Hf}$ model age between $2.65 \mathrm{Ga}$ to $2.83 \mathrm{Ga}$ (Table 6), where $\varepsilon \mathrm{Nd}(\mathrm{t})$ values are from -0.12 to -6.42 and $\mathrm{Nd}$ model ages from 2.27 to $2.54 \mathrm{Ga}$ (Table 8 ). We interpret this magma as the earlier continental magmatic arc in the Trairão region. 
After a period of quiescence, between $1940 \mathrm{Ma}$ and $1878 \mathrm{Ma}$, a new accrecional arc begins to be formed in the same tectonic environment. The form of the rise of the liquid is similar to the above with a larger contribution of crustal material, which it included the reworking of 1.94-1.96 Ga biotite granite. The crustal residence time of this second group, the Matupá Intrusive Suite, is longer than the first group and have one grantic sample with $\varepsilon \mathrm{Hf}$ $(\mathrm{t}=1856 \mathrm{Ma})$ from -4.5 to -6.5 and another sample with $\varepsilon \mathrm{Hf}(\mathrm{t}=1837 \mathrm{Ma})$ from -7.9 to -9.5 (Table 7). The Matupá granitic rocks show $\varepsilon N d(t)$ between +0.05 and -3.44 and $\mathrm{Nd}$ model ages 2.25 and $2.36 \mathrm{Ga}$ (Table 8), which can be correlated to the first granitic sample. The present $\mathrm{Nd}$ and Hf data suggest that the magma have different proportion of crustal source and mantelic source, as we can observe from the mineral chemistry and LuHf isotope data of zircons and $\varepsilon \mathrm{Hf}$ and $\varepsilon N d$ evolution diagrams (Figures 12 and 13).

At $1878 \mathrm{Ma}$, with the rise of epizonal level liquid, begins to crystallize rocks that make up the Group II, the granitic rocks of the Matupá Intrusive Suite. So occurred the second main flare-up event when the first pulse took place at $1878 \pm 7 \mathrm{Ma}$; the second pulse at $1851 \pm$ $12 \mathrm{Ma}$ and the last pulse at $1837 \pm 13 \mathrm{Ma}$ (Figure 10). Late hydrothermal fluids percolated the granites of this group, which lead to the fluid percolation and mineral precipitation, evidenced by the presence of Au mineral deposits in the deposits of Trairão Grosso and Chumbo Grosso.

Bettencourt et al. (2016), Fernandes et al. (2011) and Juliani et al. (2013, 2014) based on field work, geochemistry, geophysical studies and isotope geology indicate that the southern part of Amazonian Craton is essentially composed of two continental magmatic arcs that evolved between ca. 2.0 and $1.86 \mathrm{Ga}$, named Tapajonic Arcs, with a possible flat subduction stage, under an Archean Paleoproterozoic continent.

The conclusions of this work are partially in agreement to the above authors, however according to ours data and interpretation and based on the concepts of Bettencourt, et al. (2016), it may be considered for the study area an evolution made by the occurrence of three tapajônicos magmatic arcs with the following chronology:

Arc 1 - Cuiu-Cuiu Arc: $2.0 \mathrm{Ga}-1.98 \mathrm{Ga}$

Arc 2 - Creporizão - Nhandu Arc: 1.96 to 1.94 Ga

Arc 3 - Parauari -Matupá Arc: 1.88 to $1.84 \mathrm{Ga}$

Patiño Douce (1999) summarized clearly the current design based on experimental petrology, stating that peraluminous granites are the only granitic rocks unquestionably produced 
by pure crustal melting. This author stated that: "Except for peraluminous granites, generation of granitic magmas of every other type discussed here is associated with growth of the continental crust. This is so because basaltic components are clearly present in S-type granites, Cordilleran granites, Basalt plateau rhyolites, and, very possibly, A-type granites as well”.

Recent reviews discussing the genesis of granites also conclude that the granitic magmatism, is associated with magmatic arcs (Arndt, 2013; Brown, 2013) or A-type (Eby, 2006; Nardi \& Bettencourt, 2009), incorporates mafic magmas from the mantle. Dall'Agnol \& Oliveira (2007) demonstrated that it is possible to discriminate with geochemical parameters, the A-type oxidized granitoids and reduced; oxidized types strongly resemble the granitic calc-alkaline mature magmatic arc generally called I-type.

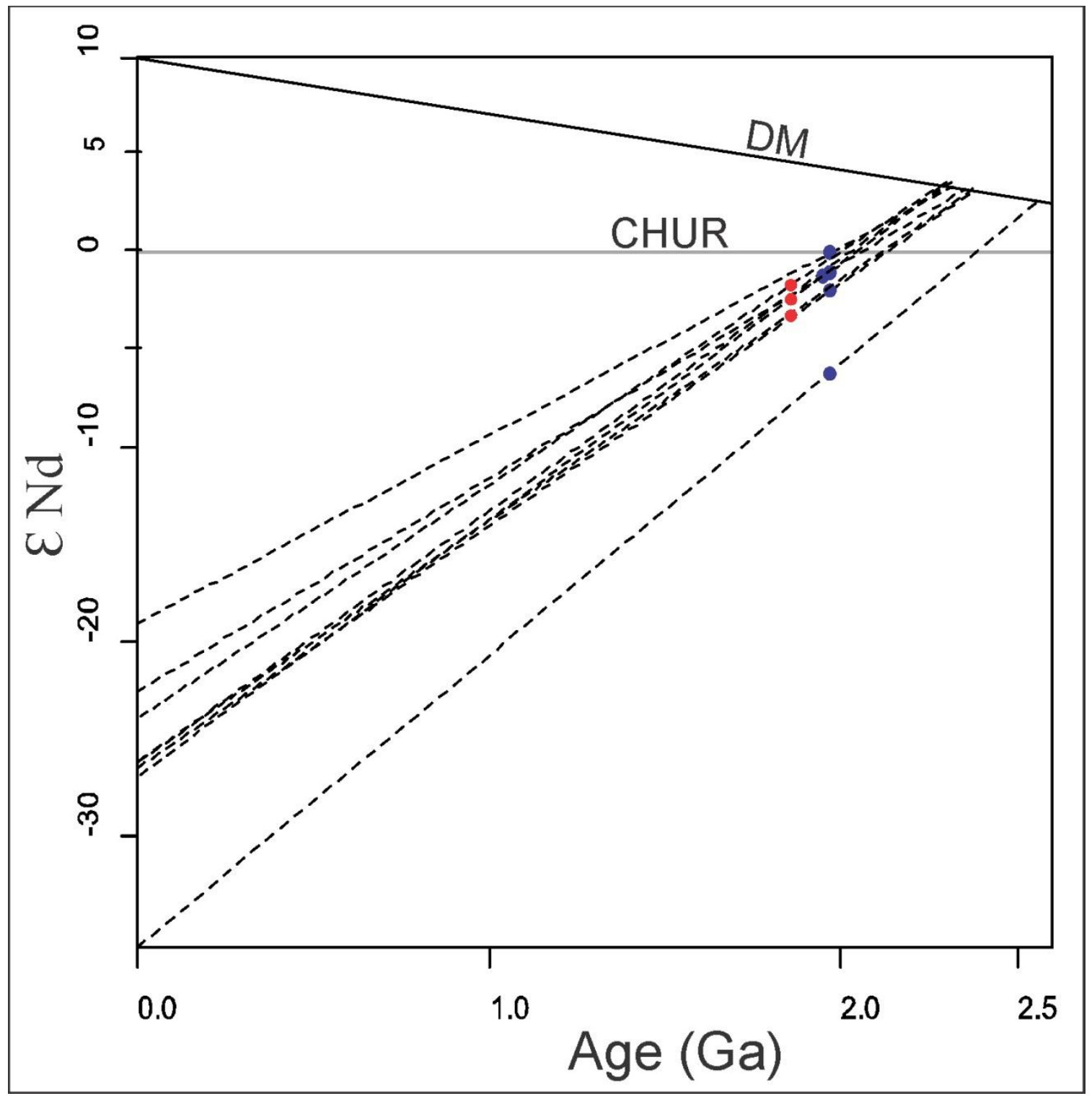

Tese de Doutorado - Mara Luiza Barros Pita Rocha 
IV Figure 13- ENd diagram for granitic rocks of the Matupá Intrusive Suites (red) and Nhandu (blue) in the Trairão region, Mundo Novo, MT.

\section{8 - CONCLUSIONS}

According on the data provided by this research, we conclude that in the Trairão Region, there are two distinct groups of granites: Group I described as Biotite Monzogranite of Matupá Intrusive Suite, geochemical characteristics of calc-alkaline to high potassium granite, metaluminous to softly peraluminous, high REE contents, with enrichment of light on heavy, negative anomaly of Eu and intrusion age of $1969 \pm \mathrm{Ma}$ (first intrusion), $1954 \pm 4 \mathrm{Ma}$ (second intrusion of biotite-granite) and $1946 \pm 21 \mathrm{Ma}$ (third intrusion of biotite granite), similar with ages described by Barros et al, 2015 to granites the Nhandu Intrusive Suite.

Group II consists of fine-grained, syenogranitic composition with geochemical pattern similar to Nhandu Granite described above. It differs in lower levels of $\mathrm{K}_{2} \mathrm{O}$, lower REE and age, ranging from $1878 \mathrm{Ma}$ to $1837 \mathrm{Ma}$, and it is here correlated to the granites of Matupá Intrusive Suite (Moura et al 1998 and Silva et al 2014). Geochemical data indicate that these rocks are of calc-alkaline series, metaluminous to lightly peraluminous and I-type granite.

Lu-Hf results show negative values for both granites (Group I and II), showing origin by crustal reworking, with significant incorporation of melts derived from melting of supracrustal rocks in the Group II, Matupá Intrusive Suite (1878 Ma - 1837 Ma). Tectonic discrimination diagrams (Pearce et al. 1984, Pearce 1996) implemented to both granites indicate mature arc environment for the evolution of these rocks, reaching the final phase of a magmatic arc.

Based on previously studies the southern part of Amazonian Craton is composed of two continental magmatic arcs, called Tapajonic Arcs, that evolved between ca. 2.0 and 1.86 Ga. Therefore, agreement with Bettencourt et al. (2016) hypothesis of and according to our interpretation, is considered for the study area an evolution following the development of three Tapajônicos Magmatic Arcs, with the respectivelly periodes of evolution; Arc 1 - Cuiu-Cuiu Arc: $2.0 \mathrm{Ga}$ - $1.98 \mathrm{Ga}$, Arc 2 - Creporizão - Nhandu Arc: 1.96 to $1.94 \mathrm{Ga}$ and finally Arc 3 Parauari -Matupá Arc: 1.88 to $1.84 \mathrm{Ga}$. 
Hydrothermal events are responsible for late fluids that percolated the granites of Group II, Matupá Intrusive Suite (first pulse at $1878 \pm 7 \mathrm{Ma}$ and last one at $1837 \pm 13 \mathrm{Ma}$ ), which lead to the fluid percolation and mineral precipitation, evidenced by the presence of Au mineral deposits in mineralized hydrothermal breccias at Trairão and Chumbo Grosso deposits, that occur in these granites. The data presented in this study reinforce the hypothesis of extending the Tapajós Domain to the east portion of Alta Floresta Gold Province in the northern state of Mato Grosso.

\section{9 - ACKNOWLEDGMENTS}

We thanks to the Postgraduate Program of Geosciences - UnB, to Laboratory of Geochronology - USP - UnB, UERJ and Curtin University of Technology, Laboratory of Electronic Microprobe UnB. The first author thanks especially to CNPq.

\section{0- REFERENCES}

Abreu M.C. 2004. Alteração hidrotermal e mineralização aurífera do depósito de Novo Mundo, região de Teles Pires - Peixoto de Azevedo, Província de Alta Floresta (MT). Trabalho de Conclusão de Curso, IG/UNICAMP, 29p.

Almeida, F.F.M. 1978. A evolução dos crátons Amazônico e do São Francisco comparada com seus homólogos do hemisfério norte. In: CONOR. BRAS. GEOL., 30. Recife, 1978. Anais... Recife, SBO. v. 6, p 2393-2407.

Almeida F.F.M.; Hasui Y.; Brito Neves B.B.; Fuck R.A. 1981. Brazilian structural provinces: an introduction. Earth Sci. Rev. 17, 1-29.

Amaral, G., 1974. Geologia Pré-Cambriana da Região Amazônica. Universidade de São Paulo, Tese de Livre Docência, São Paulo, 212 pp.

Arndt, N.T. 2013. Formation and Evolution of the Continental Crust. Geochemical Perspectives, 2(3): 405- 533.

Assis R.R. 2011. Depósitos auríferos associados ao magmatismo granítico do setor leste da Província de Alta Floresta (MT), Craton Amazônico: tipologia das mineralizações, modelos genéticos e implicações prospectivas. Dissertação de Mestrado, Universidade Estadual de Campinas, Campinas, Brasil, 428p.

Barros, M.A.S.B.; Chemale Jr, F.; Nardi, L. V. S.; Lima, E.F., 2009. Paleoproterozoic bimodal post-collisional magmatism in the southwestern Amazonian Craton, Mato Grosso, Brazil: Geochemistry and isotopic evidence. Journal of South American Earth Sciences.27:11-23.

Barros, M.A.S.; Silva, C. H.; Santos J.O.S.; Kenzo, B., 2016. Reviewing the bacaeri Complex in the southern Amazon Craton using U-Pb geochronology in zircon and monazite implications for 
the timing of mafic and granitic magmatism during the Colíder Vulcanism, In: $10^{\circ}$ SSAGI, PuertoVallarta, Mexico.

Barros M. A. S.; Barros, A. J. P.; Santos, J. O. S.; Rocha, M. L. B. P.; 2015. Extension of the Tapajós Domains to the Alta Floresta Gold Province: Evidence from U-Pb Shrimp ages of the Nhandu Intrusive Suite at 1962 and 1967 Ma In: 14 Simpósio de Geologia da Amazônia.

Bettencourt, J. S.; Juliani, C.; Xavier, R. P.; Monteiro, L. V. S.; Bastos Neto, A. C.; Kleind, E. L.; Assis, R. R.; Leite Jr, W. B.; Moreto, C. P. N.; Fernandes, C. M. D.; Pereira, V. P., 2016. Metallogenetic systems associated with granitoid magmatism in the Amazonian Craton: an overview of the present level of understanding and exploration significance. Journal of South America.

Bini, E. G.; Barros, M. A. S.; Pierosan, R.; Santos, J. O. S., 2015. Petrography and geochronology of felsic volcanic rocks at the eastern portion of Serra do Cachimbo, south-central Amazonian Craton, Brazil. In: VI Simpósio de Vulcanismo e Ambientes Associados, São Paulo. Boletim de Resumos, 2015. v. 1. p. 67.

Bizotto A.C. 2004. Mineralizações auríferas na região de Teles Pires - Peixoto de Azevedo (Mato Grosso), Província de Alta Floresta: O Depósito de Santa Helena. Trabalho de Conclusão de Curso, Instituto de Geociências, Universidade Estadual de Campinas, Campinas, 38p.

Bolhar, R.; Weaver, S.D.; Palin, J.M.; Cole, J.W.; Paterson, L.A.; 2008. Systematics of zircon crystallisation in the Cretaceous Separation Point Suite, New Zealand, using U/Pb isotopes, REE and Ti geothermometry. Contrib. Mineral. Petrol. 156, 133-160.

Brown, M. 2013. Granite: from genesis to emplacement. Geological Society of America Bulletin, 125(7-8): 1079-1113.

Chemale Jr., F.; Philipp, R.P.; Dussin, I.A.; Formoso, M.L.L.; Kawashita, K.; Bertotti, A.L.; 2011. Lu-Hf and U-Pb age determinations of Capivarita Anorthosite in the Dom Feliciano Belt, Brazil. Precambrian Research 186, 117-126.

Clemens J. D. \& Wall V. J., 1984. Origin and evolution of a peraluminous silicic ignimbrite suíte. The Violet Town Volcanics. Contributions to Mineralogy and Petrology, 88, 354-71

Clemens, J.; Watkins, J.; 2001. The fluid regime of high-temperature metamorphism during granitoid magma genesis. Contributions to Mineralogy and Petrology 140, 600-606.

Condie, K.; Beyer, E.; Belousova, E.; Griffin, W. L.; O’Reilly, S. Y.; 2005. U-Pb isotopic ages and Hf isotopic composition of single zircons: The search for juvenile Precambrian continental crust. Precambrian Research 139, 42-100.

Cordani, U. G.; Teixeira W.; 2007. Proterozoic Accretionary belts in the Amazonian Cráton. Geological Society of America, v.200, p.297-320.

Cordani U.G.; Tassinari C.C.G.; Teixeira W.; Basei M.A.S. \& Kawasita K.; 1979. Evolução Tectônica da Amazônia com base nos dados geocronológicos. In: Congresso Chileno, 2, Chile. Atas, p. 137-148.

Dall'agnol, R.; Oliveira, D. C.; 2007. Oxidized, magnetite-series, rapakivi-type granites of Carajás, Brazil: implications for classification and petrogenesis of A-type granites. Lithos, v. 93, p. 215233, 
Dezula, S. E. M.; 2016. Geoquímica e Geocronologia do Granito Aragão - Província Aurífera de Alta Floresta - Craton Amazonas - MT. Dissertação de Mestrado. Universidade Federal de Mato Grosso, 46p.

Eby, G.N. 2006. Distributions between A-type granites and petrogenetic pathways, and crustal evolution of the Amazonian Craton, Belém, PRONEX-UFPA/SBG-NO, abstracts and field trips, p.15.

Fernandes, C.M.D.; Juliani, C.; Monteiro, L.V.S.; Lagler, B.; Misas, C.M.E.; 2011. High-K calcalkaline to A-type fissure-controlled Vulcano-plutonism of the São Félix do Xingu region, Amazonian Craton, Brazil: Exclusively crustal sources or only mixed $\mathrm{Nd}$ model ages? Journal of South American Earth Sciences.

Gagnevin, D.; Daly, J.S. \& Kronz, A., 2010. Zircon texture and chemical composition as a guide to magmatic processes and mixing in a granitic environment and coeval volcanic system. Contributions to Mineralogy and Petrology, 159: 579-596.

Grimes, C.B.; Jhon, B.E.; Kelemen, P.B.; Mazdab, F.K.; Wooden, J.L.; Cheadle, M.J.; Hangoj, K.; Scwartz, J.J.; 2007. Trace element chemistry of zircons from oceanic crust: A method for distinguishing detrital zircon provance. Geology 35, 643-646.Harker A. (ed.). 1909. The natural history of the igneous rocks. NewYork, Macmillan, $384 \mathrm{p}$.

Harris, N.B.; Pearce, J.A.; Tindle, A.G.; 1986. Geochemical characteristics of collisionzone magmatism. In: Coward, M.P., Ries, A.C. (Eds.). Collision Tectonics. Geol. Soc. London, Special Publications, 19, 67-81.

Hermann, J.; Rubatto, D.; 2003. Relating zircon and monazite domains to garnet growth zones: age and duration of granulite facies metamorphism in the Val Malenco lower crust. J. Metamorph. Geol. 21, 833-852.

Hidaka, H.; Shimizu, H.; Adachi, M.; 2002. U-Pb geochronology and REE geochemistry of zircons from Palaeoproterozoic paragneiss clasts in the Mesozoic Kamiaso conglomerate, central Japan: evidence for an Archean provenance. Chem. Geol. 187, 279-293.

Hoskin, P.W.O.; Kinny, P.D.; Wyborn, D.; Chappell, B.W.; 2000. Identifying accessory mineral saturation during differentiation in granitoid magmas: an integrated approach. J. Petrol. 41, $1365-1396$.

Hoskin, P.W.O.; Schaltegger, U.; 2003. The composition of zircon and igneous and metamorphic petrogenesis. In: Hanchar, J.M., Hoskin, P.W.O. (Eds.), Zircon.: Reviews in Mineralogy and Geochemistry, 53. Mineralogical Society of America, Chantilly, Virginia, pp. 27-62.

Juliani C.; Carneiro C.C.; Carreiro-Araújo S.A.; Fernandes C.M.D.; Monteiro L.V.S.; Crósta A.P.; 2013. Estruturação dos arcos magmáticos paleoproterozóicos na porção sul do Cráton Amazônico: implicações geotectônicas e metalogenéticas. In: $13^{\circ}$ Simpósio de Geologia da Amazônia, SBG - Núcleo Norte, Belém. (CD-ROM).

Juliani, C.; Vasquez M.L.; Klein E.L.; Villas R.N.N.; Echeverri-Misas C.M.; Santiago E.S.B.; Monteiro, L.V.S.; Carneiro C. de C.; Fernandes C.M.D.; 2014. Metalogênese da Província Tapajós. In: Silva, M.G., Rocha Neto, M.B., Jost, H., Kuyumjian, R.M. (Org.) Metalogênese das Províncias Tectônicas Brasileiras, Programa Geologia do Brasil, Recursos Minerais, Serviço Geológico do Brasil, CPRM, p. 229-263. 
Klein, E. L.; and Koppe, J. C.; 2000. Chlorite geothermometry and physicochemical conditions of gold mineralization in the Paleoproterozoic Caxias deposit, São Luís Craton, northern Brazil: Geochimica Brasiliensis, v. 14, p. 219-232.

Lacerda Filho, J.V.; Souza, J.O.; Oliveira, C.C.; Ribeiro, P.S.E.; Boas P.F.V.; Albuquerque, M.C.; Frasca, A.A.S.; Borges, F.R.; Moreton, L.C.; Martins, E.G.; Camargo, M.A; Valente, C.R.; Pimentel, M.M.; Botelho, N.F. 2001. Geologia e evolução tectônica da região norte do Mato Grosso (Projeto Promin-Alta Floresta). In.: Simpósio de Geologia da Amazônia, 7, 2001, Bélem. Sessão Temática. Belém: SBG.

Lacerda Filho J.V.; Abreu Filho W.; Valente C.R.; Oliveira C.C.; Albuquerque M.C. (orgs) 2004. Geologia e Recursos Minerais do Estado de Mato Grosso. Escala 1:1.000.000. CPRM. Goiânia. 200p. CD-ROM.

Linnen, R.L., Keppler, H., 2002. Melt composition control of $\mathrm{Zr} / \mathrm{Hf}$ fractionation in magmatic processes. Geochim. Cosmochim. Acta 66, 3293-3301.

Ludwig, K.R. 2001. Using Isoplot/Ex. A geochronological toolkit for Microsoft Excel. Berkeley Geochronology Center, Special Publications No.1, Berkeley, USA.

Miguel Jr, E. 2011. Controle Estrutural das mineralizações auríferas e idades U-Pb das rochas encaixantes ao longo do Lineamento Peru-Trairão: Província Aurífera de Alta Floresta, Mato Grosso. Qualificação - Dissertação de Mestrado, Instituto de Geociências, Universidade Estadual de Campinas, 41p.

Morel, M.L.A.; Nebel, O.; Y.J. Nebel-Jacobsen, Y.J.; Miller, J.S.; Vroon, P.Z.; 2008. Hafnium isotope characterization of the GJ-1 zircon reference material by solution and laser-ablation MC-ICPMS. Chemical Geology 255, 231-235.

Moreton, L.C. \& Martins, E.G. 2003. Geologia e Recursos Minerais da Folha Vila Guarita - Folha SC.21-Z-B. Estado de Mato Grosso. Escala 1:250.000. Brasília: CPRM.

Moreton L.C. \& Martins E.G. 2005. Geologia e Recursos Minerais de Alta Floresta. Vila Guarita. Escala 1:250.000. Brasília, Serviço Geológico do Brasil, CPRM, 68 p.

Moura, M.A. 1998. O maçiço granítico Matupá no depósito de ouro Serrinha (MT): petrologia, alteração hidrometal e metalogenia. Instituto de Geociências, Universidade de. Brasília, Tese de Doutorado, 238.

Moura M.A.; Botelho N.F.; Olívio G.R.; Kyser T.K.; 2006. Granite-related Paleoproterozoic, Serrinha gold deposit, Southern Amazonia, Brazil: hydrothermal alteration, fluid inclusion and stable isotope constraints on genesis and evolution. Econ. Geol., 101:585-605.

Nakamura, N. 1974. Determination of REE, Ba, Fe, Mg, Na and K in carbonaceous and ordinary chondrites. Geochemica Acta, 38(5):757-775.

Nardi L.V.S. \& Betencourt M.F. 2009. A-type granitoids in post-collisional settings from southernmost Brazil: their classification and relationship with magmatic series. Canadian Mineralogist, 47:1493-1504.

Rubatto, D., 2002. Zircon trace element geochemistry: partitioning with garnet and the link between $\mathrm{U}-\mathrm{Pb}$ ages and metamorphism. Chem. Geol. 184, 123-138.

Paes de Barros, A.J. 1994. Contribuição à geologia e controle das mineralizações auríferas de Peixoto de Azevedo - MT. Instituto de Geociências, Universidade de São Paulo. São Paulo-SP. Dissertação (Mestrado em Geologia Econômica e Hidrogeologia), 145 p. 
Paes de Barros, A.J., 2007. Granitos da região de Peixoto de Azevedo - Novo Mundo e mineralizações auríferas relacionadas - Província Aurífera Alta Floresta (MT). Instituto de Geociências, Universidade Estadual de Campinas, Tese de Doutorado, 154p.

Patiño Douce, A.E., 1999, What do experiments tell us about the relative contributions of crust and mantle to the origin of granitic magmas?, in Castro, A., Fernandez, C., and Vigneresse, J.L., eds., Understanding Granites: Integrating New and Classical Techniques: Geological Society of London Special Publication 168, p. 77-94.

Pearce J.A.; Harris N.B.W \& Tindle A.G. 1984. Trace elements discrimination diagrams for the tectonic interpretation of granitic rocks. Journal of Petrology, 25 (4): 956-983.

Pearce, J. A. (1996). Sources and settings of granitic rocks. Episodes, 19(4), 120-125.

Peccerillo A. \& Taylor S.R. 1976. Geochemistry of Eocene calc-alkalime volcanic rocks from the Kastamonu área, northern Turkey. Contributions to Mineralogy and Petrlogy, 58: 63-81.

Pimentel, M.M. \& Botelho, N.F. 2001. Geologia e Evolução Tectônica da Região Norte do Mato Grosso (Projeto Promin-Alta Floresta). In: Simpósio de Geologia da Amazônia, 7, Belém. Resumos Expandidos... Belém: SBG - Núcleo Norte. Cd-Rom.

Pinho M.A.S.; Chemale Jr. F.; Schmus W.R.V.; Pinho F.E.C.; 2003. U-Pb and Sm-Nd evidence for 1.76-1.77 Ga magmatism in the Moriru region, Mato Grosso, Brazil: implications for province boundaries in the SW Amazon Cráton. Prec. Res., 126: 1-25.

Prado E.S.; Barros M.A.S.; Pinho F.E.C.; Pierosan R.; 2013. Granito Terra Nova - petrologia e geocronologia: um granito tipo-A da Província Aurífera Alta Floresta - Cráton Amazônico. Rev. Bras. Geoc. 43(1): 101-116.

Santos J.O.S.; Hartmann L.A.; Gaudette H.E.; Groves D.I.; Mcnaughton N.J.; Fletcher I.R.A.; 2000. A New Understanding of the Provinces of the Amazon Craton based on Integration of Field Mapping and U-Pb and Sm-Nd Geochronology. Gondwana Research, 3 (4):453-488.

Santos, J. O. S.; Groves, D. I.; Hartmann, L. A.; Moura, M. A.; Mcnaughton, N. J.; 2001. Gold deposits of the Tapajos and Alta Floresta Domains, Tapajos-Parima orogenic belt, Amazon Craton, Brazil. Mineralium Deposit, Alemanha, v. 36, n.3, p. 278-299.

Santos J.O.S. 2003. Geotectônica dos escudos das Guianas e Brasil- Central. In: Bizzi L.A., Schobbenhaus C., Vidotti R.M., Gonçalves J.H. (eds). Geologia, tectônica e recursos minerais do Brasil. CPRM. Brasília. CD-ROM.

Santos J.O.S.; Breemen O.B.V.; Groves D.I.; Hartmann L.A.; Almeida M.E.; McNaughton N.J.; Fletcher I.; 2004. Timing and evolution of multiple Paleoproterozoic magmatic arcs in the Tapajós Domain, Amazon Craton: constraints from SHRIMP and TIMS zircon, baddeleyite and titanite U-Pb geochronology. Precambrian Research, 131: (73-109).

Santos J.O.S.; Hartmann L.A.; Faria M.S.; Riker S.R.; Souza M.M.; Almeida M.E.; McNaughton N.J.; 2006. A compartimentação do Cráton Amazônico em províncias: avanços ocorridos no período de 2000-2006. In: Simpósio de Geologia da Amazônia, 9. Seção Temática III, Belém, Pará. CD-ROOM.

Santos, J. O. S.; Rizzotto, G.J.; Potter, P.E.; McNaughton, N.J.; Matos, R.S.; Hartmann, L.A.; Chemale Jr., F.; Quadros, M.E.S. 2008. Age and autochthonous evolution of the Sunsás Orogen in West Amazon Craton based on mapping and U-Pb geochronology. Precambrian Research, v. 165, p. 120-152. 
Santos, J. O. S.; Barros, M. A.; Barros,A. J. P.; McNaughton N.; 2015. Datação de Titanita usando a relação ${ }^{208} \mathrm{~Pb} /{ }^{232} \mathrm{Th}$ : Caso do Gnaisse Gavião, Província Aurífera Alta Floresta In: $14^{\circ}$ Simpósio de Geologia da Amazônia.

Shand, S.J., 1943. Eruptive Rocks.Their Genesis, Composition, Classification, and Their Relation to Ore-Deposits with a Chapter on Meteorite. New York: John Wiley \& Sons.

Silva M. G. \& Abram M.B. (orgs) 2008. Projeto Metalogenia da Província Aurífera Juruena - Teles Pires, Mato Grosso. Informe de Recursos Minerais, Série Ouro, 16. CPRM. Goiânia. 212p.

Silva, F. R.; Barros, M. A. S.; Pierosan, R.; Pinho, F. E. C.; Rocha, M. L. B. P.; Vasconcelos, B. R.; Dezula, S. E. M.; Tavares, C.; Rocha, J.; 2014. Geoquímica e geocronologia U-Pb (SHRIMP) de granitos da região de Peixoto de Azevedo: Província Aurífera Alta Floresta, MT, Brazilian Journal of Geology, 44(3): 433-455.

Silva, G.H. 1974. Esboço Geológico de parte da Folha Sc.21. Juruena. In: Congresso Brasileiro de Geologia, 28, Porto Alegre. Anais. Porto Alegre: SBG,1974, V.4, P.309-320.

Souza A.M.M., Faria C.A.S., Landim J.P.P., Leal J.W.L. 1979. Projeto São Manoel. DNPMCPRM, Relatório de progresso, Belém, 46p.

Souza, J.O.; Frasca, A.A.S.; Oliveira, C.C., 2004. Projeto Província Mineral de Alta Floresta. Geologia e Recursos Minerais das folhas Rio São João da Barra (SC.21-V-D), Alta Floresta (SC.21-X-C), Ilha 24 de Maio (SC.21-Z-A); Vila Guarita (SC.21-ZB), estados de Mato Grosso e Pará. Escala 1:250.000. Brasília: CPRM.

Souza J.O.; Frasca A.A.S.; Oliveira C.C.; (orgs.) 2005. Geologia e Recursos Minerais da Província Mineral de Alta Floresta. Relatório Integrado. Folhas SC.21-V-D, Rio São João da Barra; SC.21-X-C - Alta Floresta; SC.21-Z-A - Ilha 24 de Maio, SC.21-Z-B - Vila Guarita. Escala 1:250.000. CPRM. Brasília. CR-ROM.

Stevens, G., and Clemens, J.D., 1993, Fluid-absent melting and the roles of fluids in the lithosphere: A slanted summary?: Chemical Geology, v. 108, p. 1-17, doi: 10.1016/0009-2541 (93)903149.

Tassinari C.C.G. \& Macambira M.J.B. 1999. Geochronological Provinces of the Amazonian Cráton. Episodes, 22 (3):174-182.

Tassinari C.C.G. \& Macambira M.J.B. 2004. A evolução tectônica do Cráto Amazônico. In: Matasso-Neto V., Bartorelli A., Carneiro C.D.R, Brito-Neves B.B. (eds.) Geologia do continente sul-americano: Evolução da obra de Fernando Flávio Marques de Almeida. Beca, São Paulo, 673p.

Teixeira, W.; Ávila, C.A.; Dussin, I.A.; Corrêa Neto, A.V.; Bongiolo, E.M.; Santos, J.O.; Barbosa, N.S.; 2015. A juvenile accretion episode $(2.35-2.32 \mathrm{Ga})$ in the Mineiro belt and its role to the Minas accretionary orogeny: Zircon U-Pb-Hf and geochemical evidences. Precambrian Research , 256, p. 148-169.

Teixeira, W.; Tassinari, C.; Cordani, U.; Kawashita, K.; 1989. A review of the geochronology of the Amazonian Cráton: Tectonic implications. Precambrian Research, v. 42, n.3/4, p. 213-227.

Thompson R.N. 1982. Magmatism of British Tertiary volcanic province. Scottish Journal of Geology, 18:49-107.

Trevisan, V. G.; Xavier, R. P.; Rodrigues, R. M.; Assis, R.R.; Paes de Barros, A. J.; 2012. Regime de fluidos em depósitos de ouro associados a intrusões paleoproterozóicas da Província 
Aurífera de Alta Floresta (MT), Cráton Amazônico. In: XX Congresso Interno de Iniciação Científica da Unicamp. 2012, Campinas - SP. XX Congresso Interno de Iniciação Científica da Unicamp.

Trevisan, V. G. 2015. Estudo comparativo entre mineralizações filonares de $\mathrm{Au}+\mathrm{Cu}$ e $\mathrm{Au}+$ metais base do setor leste da Província Aurífera de Alta Floresta (MT) Cráton Amazônico. Instituto de Geociências, Universidade Estadual de Campinas, Dissertação de Mestrado.

Villaseca, C.; Martín-Romera, C.; De la Rosa, J.; Barbero, L., 2003. Residence and redistribution of $\mathrm{REE}, \mathrm{Y}, \mathrm{Zr}$, Th and $\mathrm{U}$ during granulite-facies metamorphism: behaviour of accessory and major phases in peraluminous granulites of central Spain. Chem. Geol. 200, 293-323.

Williams, I.S. 1998. U-Th-Pb geochronology by ion microprobe. In: McKibben, M.A., Shanks III,W.C., Rydley,W.I. (Eds.), Applications of Microanalytical Techniques to Understanding Mineralizing Processes, Rev. Econ. Geol. 7, 1-35.

Williams,I.S. and Meyer,C. 1998. U-Pb geochronology of zircons from lunar breccia 73217 using a sensitive high mass-resolution ion microprobe. J. Geophys. Res. 89: B525-B534.

Woodhead, J.D.; Hergt, J.M. 2005. A preliminary appraisal of seven natural zircon reference materials for in situ hf isotope determination. Geostandards and Geoanalytical Research 29, 183-195.

Xiang, W.; Griffin, W.L.; Jie, C.; Pinyon, H.; Xiang, L.; 2011. U and Th Contents and Th/U Ratios of Zircon in Felsic and Mafic Magmatic Rocks: Improved Zircon-Melt Distribution Coefficients. Acta Geol. Sin. 85, 164-174. 


\section{CAPÍTULO - V \\ II ARTIGO}




\title{
ARTIGO II:
}

"AGE OF GOLD MINERALIZATION HOSTED BY OROSIRIAN GRANITE AT THE TRAIRÃO REGION - ALTA FLORESTA GOLD PROVINCE (AMAZONIAN CRATON): CONSTRAINTS FROM U-Th-Pb SHRIMP DATING OF HYDROTHERMAL MONAZITE“"

\author{
Authors: \\ Mara Luiza Barros Pita Rocha ${ }^{1}$ \\ Farid Chemale Junior ${ }^{1}$ \\ João Orestes Schneider Santos ${ }^{2}$ \\ Marcia Aparecida de Sant'Ana Barros ${ }^{3}$ \\ Francisco Egídio Cavalcante Pinho ${ }^{3}$ \\ Paulo Cesar Correa da Costa ${ }^{3}$ \\ Élzio Barbosa ${ }^{3}$ \\ 1 - Universidade de Brasília - UnB, Brasília, Brazil \\ 2 - University of Western of Australia - UWA, Perth, Australia \\ 3 - Universidade Federal do Mato Grosso - UFMT, Cuiabá, Brazil
}

Artigo a ser submetido à revista "Journal of Brazilian Geology" 


\title{
THE AGE OF GOLD MINERALIZATION HOSTED BY OROSIRIAN GRANITE AT TRAIRÃO DEPOSIT - ALTA FLORESTA GOLD PROVINCE (AMAZONIAN CRATON): CONSTRAINTS FROM U-Th-Pb SHRIMP DATING OF HYDROTHERMAL MONAZITE
}

Mara Luiza Barros Pita Rocha ${ }^{1}$ - Farid Chemale Junior ${ }^{1,4}$ - João Orestes S. Santos ${ }^{2}$ - Marcia Aparecida de Sant'Ana Barros ${ }^{3}$ - Francisco Egídio Cavalcante Pinho ${ }^{3}$ - Paulo Cesar Correa da Costa $^{3}$ - Élzio Barbosa ${ }^{3}$

1- Programa de Pós-Graduação em Geologia, Universidade de Brasília - UnB, Brasília-DF, Brazil

2- University of Western of Australia - UWA, Perth, Australia

3- Deaprtamento de Geologia, Universidade Federal do Mato Grosso - UFMT, Cuiabá-MT, Brazil

4- Universidade do Rio dos Sinos, São Leopoldo-RS, Brazil

\begin{abstract}
The Trairão gold deposits are located in the Peixoto de Azevedo domain as part of Alta Floresta Gold Province, Amazonian Craton, which are hosted by 1960 Ma to 1840 Ma arc-related granites. Two important $\mathrm{Au}$ deposits occur in the region, the Trairão and Chumbo Grosso, that are structurally controlled by $\mathrm{N} 80^{\circ} \mathrm{W}-\mathrm{S} 80^{\circ} \mathrm{E}$, trending lineament and associated with quartz veins and disseminate sulphides in a strong phyllic alteration zone of the host granite. Two samples from the ore alteration zone and host rocks showing hydrothermal and magmatic features were chosen for isotopic studies. Hydrothermal monazite crystals, formed during the Au-mineralization event, occur as small anhedric mineral filling fractures or as isolated grains associated with $\mathrm{Ag}, \mathrm{Au}$, molybdenite, barite, pyrite, galena and sphalerite. The hydrothermal monazites contain very low $\mathrm{U}$, relatively low $\mathrm{Th}$, and moderate $\mathrm{Nd}$ and $\mathrm{La}$ contents. SHRIMP U-Th-Pb dating of these crystals yielded an age of $1812 \pm 18 \mathrm{Ma}$ for the Trairão and $1825 \pm 27 \mathrm{Ma}$ for the Chumbo Grosso Au-deposit, whereas the magmatic zircon grains of rock hosting mineralization are dated at 1851 $\pm 12 \mathrm{Ma}$. The obtained results suggest that the main metallogenetic event occurred at 1812-1825 Ma, post-dating the 1851 Ma host granite.
\end{abstract}

Keywords: Hydrothermal monazite, U-Th-Pb SHRIMP dating, Au-bearing deposits, Alta Floresta Gold Province, Amazonian Craton. 


\section{1-INTRODUCTION}

The Amazonian Craton (AC) (Figure 1), formerly considered by Amaral (1974) and Almeida et al. (1981) as a large Archean platform and partially reworked and reactivated during the Proterozoic with expressive anorogenic felsic magmatism. However, the continuous acquisition of geochronological data (mostly by $\mathrm{Rb}-\mathrm{Sr}$ and $\mathrm{U} / \mathrm{Pb}$ zircon methods) point out that the AC grew through continued Proterozoic collisional/accretionary processes driven by northeasterly B-type subductions, surrounding an Archean core (Cordani et al. 1979) Teixeira et al., 1989; Tassinari and Macambira, 2004; Cordani and Teixeira, 2007).The same tectonic structure was reinforced, with modifications of some provinces, by Santos et al. (2000) and Santos (2003), based on U/Pb SHRIMP zircon and ID-TIMS data from a few hundred samples, as well as $\mathrm{Pb} / \mathrm{Pb}$ evaporation. More recently, Fernandes et al. (2011) and Juliani et al. (2013, 2014) have suggested lack of island arc terranes in the Tapajos Domain and recognize two continental magmatic arcs that evolved between ca. 2.0 and $1.86 \mathrm{Ga}$, named Tapajonic Arcs, with a possible flat subduction stage, under an Archean Paleoproterozoic continent.

Large gold and copper-gold deposits are found within the Proterozoic Tapajos and Alta Floresta provinces, which are marked by successive arc-related magmatism with steady-state and flare-up stages similar as those stages for the Sierra Nevada Batholith, presented by Paterson and Ducea (2015). The Alta Floresta Gold Province, which is the focus of present work, is a very important gold producer province located in the north part of Mato Grosso, Brazil. This province occurs as WNW-ESE trending structure with multiple granitic intrusions many times associated with felsic volcanism as part of the Tapajos-Parima and Rondonia-Juruena arcs. The later granitic emplacement occurred at $1782-1760 \mathrm{Ma}$ (U-Pb), Pinho et al 2003, Neder et al. (2002) and these intrusions are interpreted to be post-kinematic. The other multiple granitic intrusions formed between $2000 \mathrm{Ma}$ to $1800 \mathrm{Ma}$ in three main flare-up stages (main period of crustal growth) at 1.96-1.94 Ga, 1.86-1.84 Ga and 1.82-1.80 Ga. Here is very important to clarify that the region is part of two provinces: the eastern area is the continuation of the Tapajós-Parima Province and named Peixoto Azevedo Domain (Santos et al., 2000). Here several units correlate to the Tapajós Domain such as Cuiú-Cuiú Complex (ages in the 2030-2000 Ma range); Creporizão Suite (1955$1980 \mathrm{Ma}$ ), such as the Nhandu Intrusive Suite of Mato Grosso; and the Maloquinha Suite (1870$1860 \mathrm{Ma})$, such as the Matupá granite. The western part of this gold province is formed by 
younger rocks (Statherian to Late Orosirian units) related to the Juruena Domain of the RondôniaJuruena Province. Here the basement is about 100 millions of years younger (1820-1830 Ma) than the basement of the Tapajós and Peixoto de Azevedo Domains (2030-2000 Ma). The Peixoto de Azevedo Domain has intrusions of Statherian granitoids and was affected by the major events related to the evolution of the Rondônia-Juruena orogen.

The Au-mineralization is historically mined in the Alta Floresta Province since 1970. From 1970 to 1999 more than 160 ton of gold was extracted from this province. Recently, polymetallic deposits have been discovered with some similarities to $\mathrm{Au}, \mathrm{Au} \pm \mathrm{Cu}$ porphyry systems (Moura, 2002), as those deposits of Serrinha deposit associated with Matupá Massive Granitic. Ultimately, polymetallic epithermal of intermediate sulfidation were defined in the Franscico deposit (Assis, 2012). Neder (2000) described a polymetallic deposit ( $\mathrm{Zn}, \mathrm{Pb}, \mathrm{Cu}-\mathrm{Ag}, \mathrm{Au}$ ) hosted by felsic volcanic rocks located in the northwestern portion of Mato Grosso, Aripuanã - named Expedito Deposit. $\mathrm{U}-\mathrm{Pb}$ age in zircon for this volcanic indicate an age of $1762 \pm 6 \mathrm{Ma}$ which it is interpreted as the age of the mineralization in an intra-continental Mesoproterozoic rift setting.

Many geochronological survey has been done in this area with main focus on $\mathrm{U}-\mathrm{Pb}$ zircon dating by different methods as ID-TIMS, LA-ICPMS and SHRIMP. However, few dating was carried out to figure out the hydrothermal events. Assis et al. (2015) present of ${ }^{40} \mathrm{Ar} /{ }^{39} \mathrm{Ar}$ ages from sericite samples from sericitic halo at Francisco auriferous deposit that vary from $1.779 \pm 6.2$ to $1.777 \pm 6.3 \mathrm{Ma}$. New Re - Os dating in pyrite and molybdenite suggests three auriferous events to the Alta Floresta Gold Province; the first in 1.98-195 Ga, the second in 1.87-1.85 Ga and the last one in $1.78-1.77 \mathrm{Ga}$.

Recently data obtained by Serrrato (2014) classified the Juruena deposit as an intrusionhosted gold deposit, hosted by granitic rocks of Paranaíta Intrusive Suite (1819 to $1793 \mathrm{Ma}$ ) which is crosscut by different sets of mafic intrusions. Serrato (2014) divided the hydrothermal alteration into five stages, from early to late: (1) potassic alteration with quartz + sulfides and quartz + chlorite + fluorite + sulphides veins, (2) sericitic alteration with quartz + molybdenite + pyrite veins with K-feldspar halo and quartz + calcite + chlorite veins with sericitic halo, (3) carbonatization with calcite + fluorite + sulphides veins, (4) silicification, pervasive and in veins, and (5) propylitic alteration with epidote and calcite veins, and recognized the gold deposit associated with the alteration stages 1 and 3. Isotopic data from molybdenite that coexists with Au-bearing pyrite from alteration stage 2, revelead a Re-Os model age of $1805 \pm 7 \mathrm{Ma}$. 


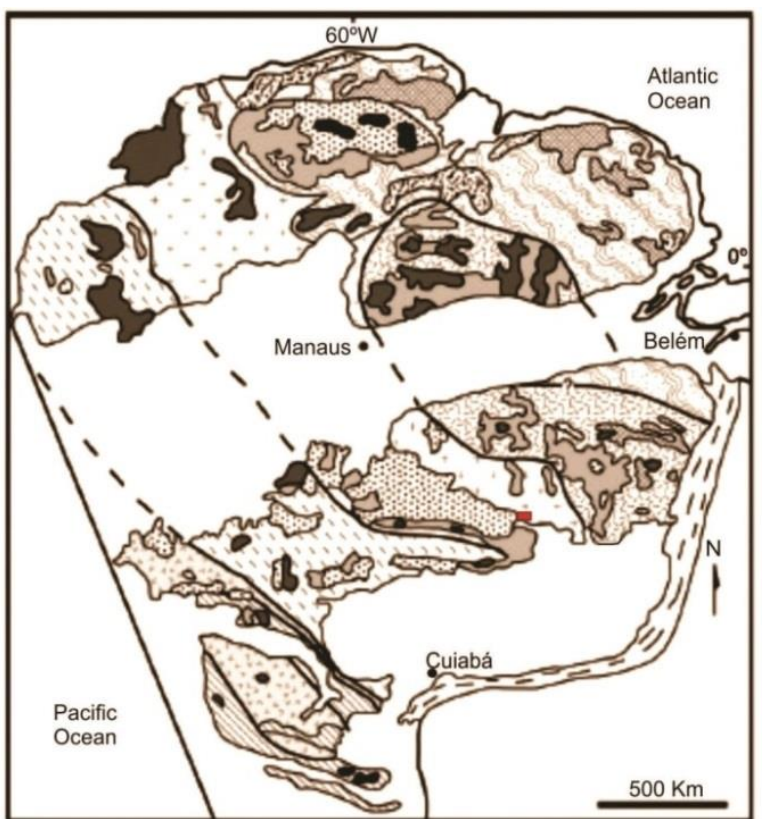

LEGEND
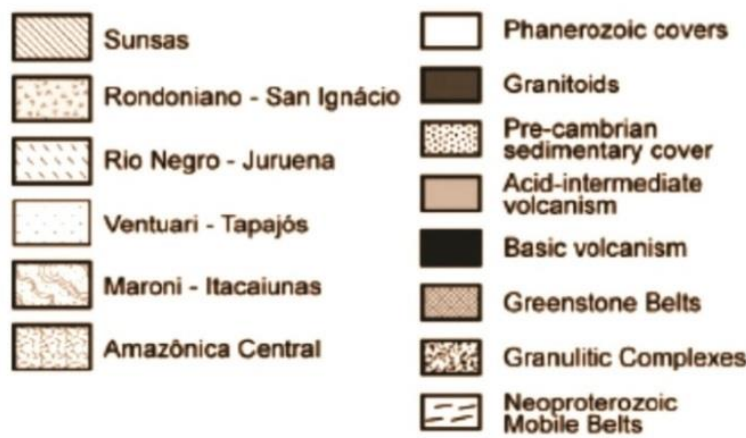

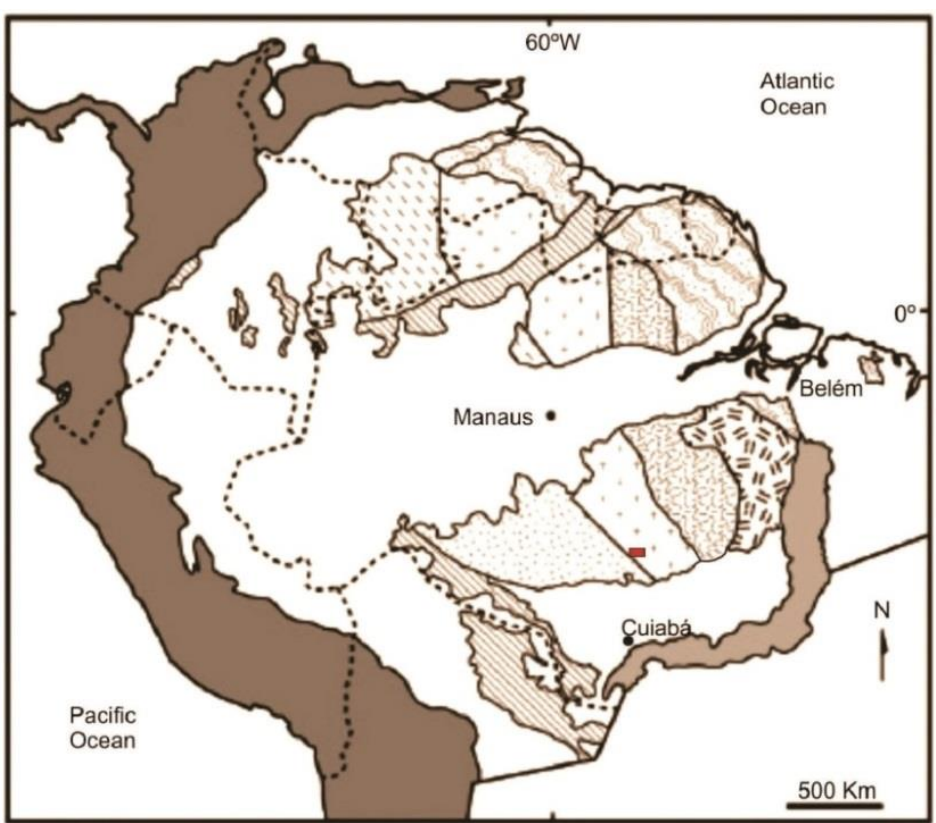

LEGEND

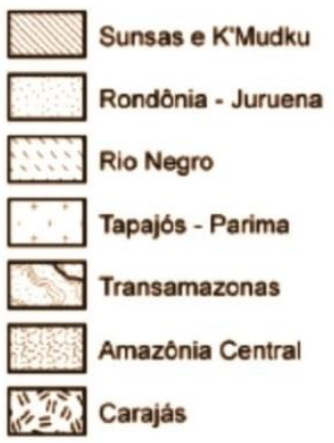

V Figure 01 - Location of the geochronological and tectonic provinces of the Amazonian Craton according the: (A) Tassinari \& Macambira (1999) and (B) Santos et al. (2008) models.

Such scarcity of age constrains of hydrothermal events and therefore the $\mathrm{Au}-\mathrm{Cu}$ polymetallic mineralization is due to lack of hydrothermal mineral formed during the mineralization process, which they yield reliable dating. So the mean goal of this paper is bring out new ages of gold deposit from Alta Floresta Gold Province (AFGP), using U-Th-Pb in hydrothermal monazite from Trairão region, NW portion of AFGP.

\section{2-GEOLOGICAL SETTING}


Alta Floresta Gold Province (Dardene and Schobenhaus 2001) also referred as the Alta Floresta Gold Province (AFGP) (Santos et al, 2004) or Juruena - Teles Pires Gold Province (Silva \& Abram, 2008) extends over $500 \mathrm{~km} \mathrm{NW-SE} \mathrm{in} \mathrm{the} \mathrm{southeast} \mathrm{portion} \mathrm{of} \mathrm{the} \mathrm{Amazonian}$ Craton (Souza et al., 2005). The province is composed of Tapajós - Parima (2.0-1.88 Ga) and Rondonia - Juruena (1.82-1.54 Ga) geochronological provinces (Santos, 2003). These provinces were interpreted as products of successive arc accretion which envolved important addition of juvenile material as well as a reworking of older continental crust (Tassinari \& Macambira, 1999; Santos et al. 2000).

The AFGP is composed mainly of high potassium calc-alkaline granites (ranging from granodiorites to alkaline- feldspar granite). Some fragments of iron formation associated with mafic rocks occurs locally and it was named as Bacaeri-Mogno Complex interpreted for some authours as an evidence of suture zone (Duarte et al. 2015). The oldest granites in the region can be grouped as Cuiú-Cuiú Complex (2.1-1.99 Ga) which is represented in the area by tonalitic to granitic gneiss (Souza et al, 2005, Santos et al, 2004). Several high-k granites are present in the area, as follow: (i) Nhandu Intrusive Suite (1.96 Ga); (ii) Matupá Intrusive Suite (1.87 Ga), (iii) Juruena Intrusive Suite (including Paranaíta, Cristalino and Apiacás Granite) (1.82 a 1.79 Ga), and (iv) large felsic volcanism included into two Groups Colíder $(1.78 \mathrm{Ga})$ and Serra do Cachimbo Formation (1.76 Ga) (Bini et al. 2015). Anorogenic Granite with $1.75 \mathrm{Ga}$ is named as Teles Pires Intrusive Suite. The figure 1 shows a geologic map of SE portion of the AFGD with insert of studied area. 


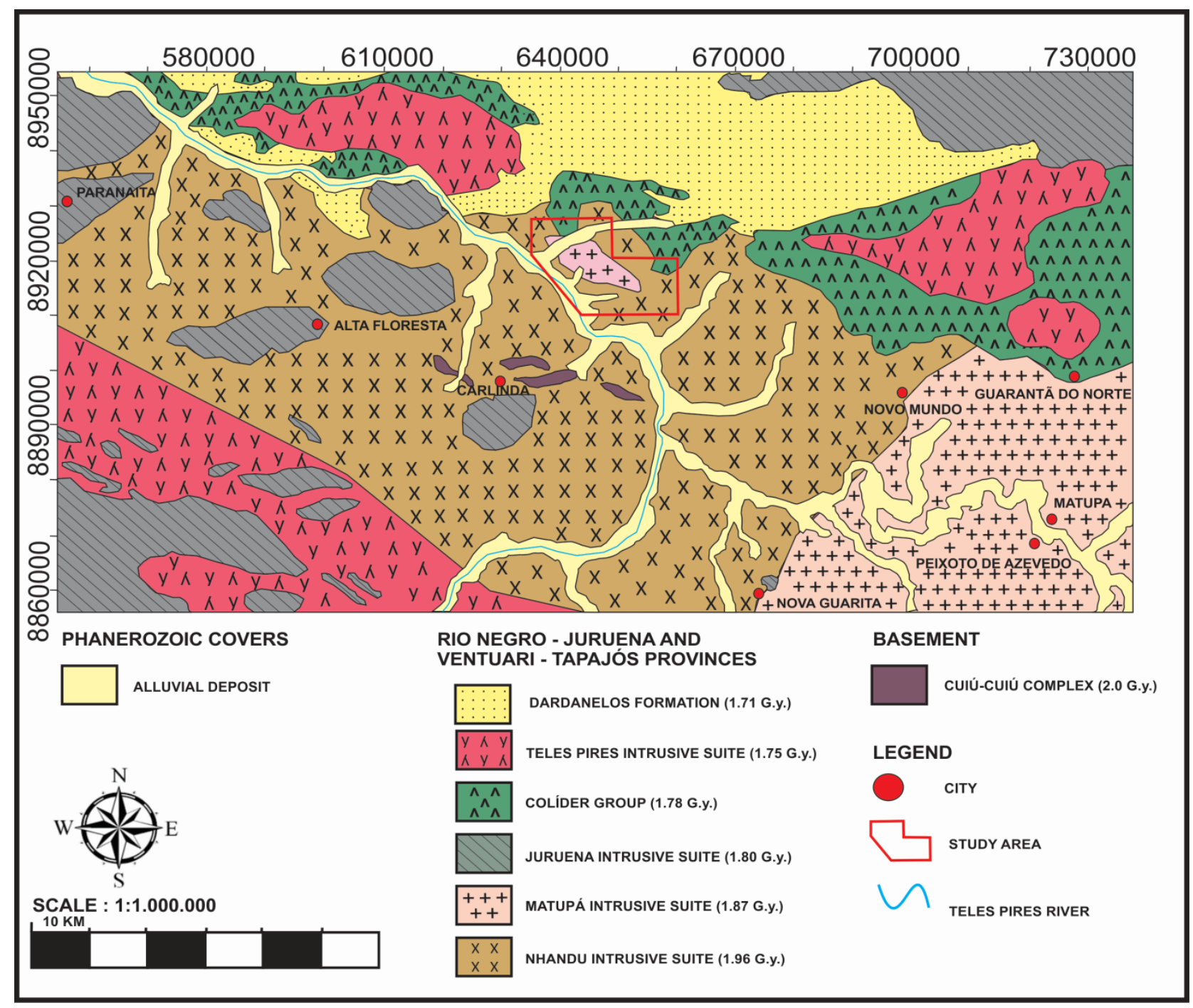

V Figure 02 - Geological map of part of Alta Floresta Gold Province. The insert is the study area where the Trairão Au-deposits, modified from Miguel Jr. (2011)

\section{1- CARACTERIZATION OF THE ANALYSED SAMPLES}

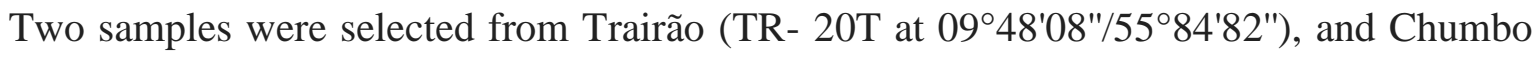
Grosso (TR- $18 \mathrm{~S}$ at $09^{\circ} 47^{\prime} 36^{\prime \prime} / 55^{\circ} 40^{\prime} 43^{\prime}$ ) deposits. The TR- 20T sample was characterized as an potassium rich granite composed of feldspar, plagioclase and quartz with advanced degree of hydrothermal alteration quite where sericitization of feldspars is the most abundant. Monazites recognized by SEM (Scanning Electron Microscope) are associated with barite, sphalerite, galena and pyrite. Some inclusions of gold and silver, sheelite and rare uraninite were observed. Magnetite has a low incidence (less than 5\%). The TR-18S sample is an granite containing large 
silicification and clasts of potassic feldspar. Titanite and magnetite were observed as accessory minerals and small crystals of molybdenite.

The contact areas are characterized by intense silica injections in various directions, strongly conditioned the porphyry contact zones. These areas are characterized by the development of dense stockwork systems, hydrothermal breccias cemented by quartz with up to 3 meters wide and preferred direction $\mathrm{N} 82^{\circ} \mathrm{E} / \mathrm{S} 77^{\circ} \mathrm{E}$, coloformes textures, milky quartz veins and boxworks sulfide, preferably pyrite and chalcopyrite.

In the silicified mineralized portions, that it corresponds to the most important hydrothermal stage, there is the occurrence of cracks related to hydraulic contact areas with subvolcanic granite, enabling percolation of hydrothermal fluid and probably the precipitation of sulfides. Based on field observations with petrographic analysis, the igneous textures suggest that the granite massif in question would be saturated in a fluid phase in shallow crustal levels (Candela 1997).

\section{2- HYDROTHERMAL ALTERATION AND BRECCIA TYPE}

The Trairão (TR-20 T) and Chumbo Grosso (TR-18 S) orebodies are hosted preferentially by breccias. These breccias are mainly polymictic and classified as quartz breccia, rich in magnetite, and sericite breccia. The angular to sub rounded rock fragments range from millimeters up to few centimeters of diameter. The fragment to matrix ratio is highly variable, reflecting ratios fluid - rock and mechanical processes that occurred during the brecciated. The fragments are compounds of quartz and wallrock (biotite syenogranite) cemented by silica and alteration minerals. At Trairão orebodies, mineralization is hosted preferentially by quartz vein breccias. These breccias exhibit a fine grained matrix of subhedral magnetite cemented by silica together with sulfides (pyrite, galena, chalcopyrite, sphalerite), monazite, barite rarely uraninite and xenotime. Several vein types crosscut the host rock however comb structure is common. Venules and / or pods filled with quartz \pm sulphide are common in this type of hydrothermal alteration. Scanning electron microscopy (SEM) of quartzo breccia has revealed pyrite, aggregate of monazite and barite and sheelite. This mineral assemblage defines the hydrothermal Au enriched zone in this granite. 


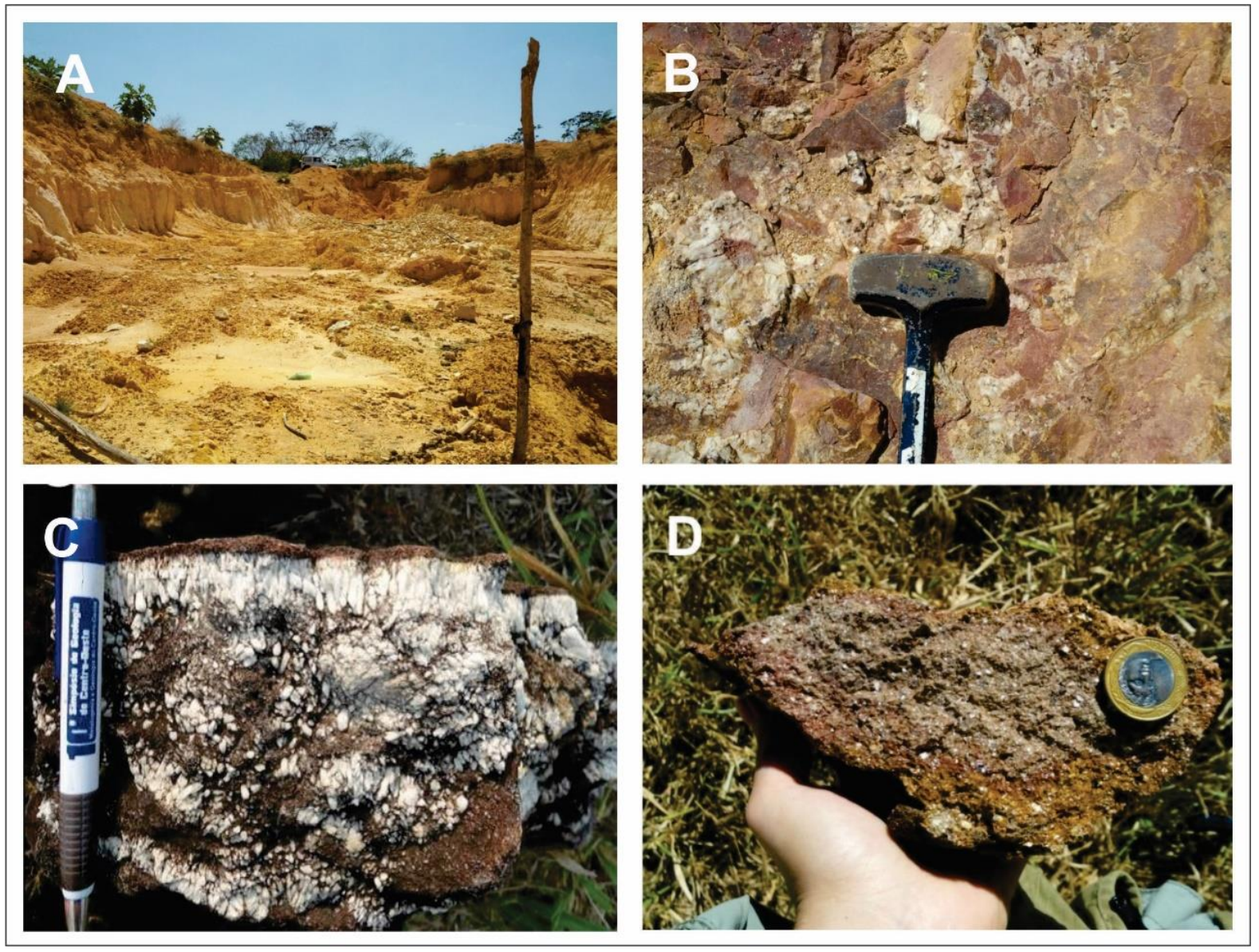

V Figure 03 - (A) Panoramic view of the Trairão deposit region; (B) Mineralized veins crosscut the host rock, hydrothermal breccia in biotite syenogranite; (C) comb structure quartz-vein, that crosscut the granite Trairão; (D) detail of mineralized sample.

At Chumbo Grosso orebodies, gold mineralization is associated with diagnostic postmagmatic alteration. Secundary alteration pervasive (sericite + chlorite + calcite + pyrite) is the most widespread hydrothermal alteration type in the Chumbo Grosso deposit and the occurrence of sulfites is clear associate with this alteration. Therefore, replacement of igneous plagioclase to carbonate (saussuritization) is frequent and breakdown of muscovite and biotite primary to fengite and well developed chlorite also occur. The breccia matrix also is report to include fine grained of quartz and siderite. Scanning electron microscopy of sericite breccia has revealed the presence of hydrothermal minerals as barite, pyrite and ilmenite and monazite include in sphalerite, plus gold disseminated (Figure 4), venules of epidote and / or calcite represent the later hydrothermal event system. 


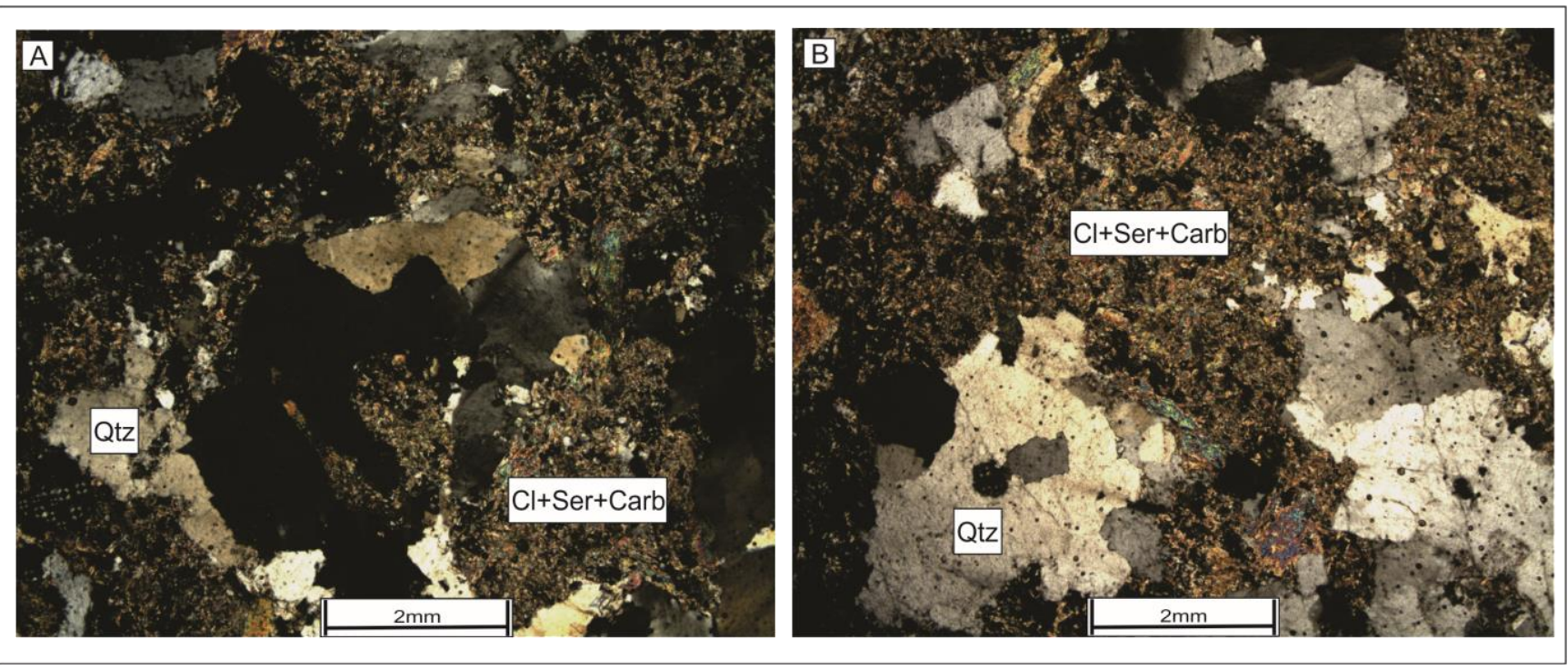

V Figure 04 - (A-B) secondary pervasive alteration (sericitic) in plagioclase minerals (sample TR-08 S), characterized by chlorite + sericite + carbonate + epidote \pm pyrite. (crossed nicols).

\section{3-ANALITICAL PROCEDURES}

Monazite (LREE, Th, U), $\mathrm{PO}_{4}$ is very resistant to radiation damage (e.g., Meldrum et al. 1998, 1999, 2000; Nasdala et al. 1999), and U-Th-Pb resetting by solid diffusion is negligible (Cherniak et al. 2004) and because of these properties represents an excellent mineral for combined ${ }^{232} \mathrm{Th} /{ }^{208} \mathrm{~Pb},{ }^{238} \mathrm{U} /{ }^{206} \mathrm{~Pb}$ and ${ }^{235} \mathrm{U} /{ }^{207} \mathrm{~Pb}$ isotopic dating of geologic processes (e.g., Parrish 1990). This dating approach is especially useful in polymetamorphic regions, where rockforming monazite generally shows complex, small-scale growth zoning (Berger et al. 2006; Krenn et al. 2011a; Schulz and Von Raumer 2011). The other positive characteristics about monazite use in ages determinations is that common $\mathrm{Pb}$ has a negligible influence on the age determination. Because hydrothermal monazite is commonly very small size in contrast to monazite from metamorphic rocks, the use of SHRIMP II is the best way to get good results, in situ U-Pb dating of monazite may become an important chronometer for hydrothermal event.

Monazites were first observed in thin section from ore bearing samples. Then they were confirmed using Tescan Eletronic Microscopy, cheking with EDS. After confirmation the samples were drilled removing the monazite from polished the thin sections and mounted in epoxy disk. The monazite $\mathrm{U}-\mathrm{Pb}$ standard $\mathrm{OX} 1\left({ }^{206} \mathrm{~Pb} /{ }^{238} \mathrm{U}\right.$ age $\left.=520 \pm 8 \mathrm{Ma}\right)$ was placed in a separate mount. 


\section{1- MINERAL CHEMISTRY}

Compositional analyses were acquired on an electron microprobe (JEOL JXA8530F) equipped with 5 tunable wavelength dispersive spectrometers. Operating conditions were 40 degrees takeoff angle, and a beam energy of $20 \mathrm{keV}$. The beam current was $130 \mathrm{nA}$, and the beam diameter was fully focussed. Elements were acquired using analyzing crystals LiF for Dy, Pr, Er, Nd, Gd, Sm, Yb, Eu, PETH for Th, Pb, PETJ for U, La, Y, Ce, Ca, P, PETH for Th, Pb, and TAP for Si.

The standards were Crocoite for $\mathrm{Pb}$ ma, Monazite for $\mathrm{Th}, \mathrm{Eu}$, Wollastonite for $\mathrm{Ca}, \mathrm{Si}$, Uraninite for $\mathrm{U}, \mathrm{YPO}_{4}$ for $\mathrm{P}, \mathrm{Y}, \mathrm{La} \mathrm{PO}_{4}$ for $\mathrm{La}, \mathrm{CePO}_{4}$ for $\mathrm{Ce}, \mathrm{PrPO}_{4}$ for $\mathrm{Pr}, \mathrm{NdPO}_{4}$ for $\mathrm{Nd}$, $\mathrm{SmPO}_{4}$ for $\mathrm{Sm}, \mathrm{GdPO}_{4}$ for $\mathrm{Gd}, \mathrm{DyPO}_{4}$ for $\mathrm{Dy}, \mathrm{ErPO}_{4}$ for $\mathrm{Er}$, and $\mathrm{YbPO}_{4}$ for $\mathrm{Yb}$.

The counting time was 20 seconds for $\mathrm{Pr}, \mathrm{Nd}, \mathrm{Gd}, \mathrm{Sm}, \mathrm{Yb}, \mathrm{La}, \mathrm{Ce}, \mathrm{Ca}, \mathrm{P}, \mathrm{Eu}, 40$ seconds for $\mathrm{Dy}, \mathrm{Y}, \mathrm{Er}, 100$ seconds for $\mathrm{Si}$, and 200 seconds for $\mathrm{Pb}$, Th, U. The MAN background intensity data was calibrated and continuum absorption corrected for $\mathrm{U}$ ma, $\mathrm{Si}, \mathrm{Pb}, \mathrm{Th}, \mathrm{Dy}, \mathrm{Pr}, \mathrm{Er}, \mathrm{Nd}, \mathrm{Gd}$, Sm, Yb, La, Y, Ce, Ca, P, Eu. Unknown and standard intensities were corrected for deadtime.

Interference corrections were applied to $\mathrm{U}$ for interference by $\mathrm{Th}, \mathrm{Pr}, \mathrm{Sm}, \mathrm{Ce}$, and to $\mathrm{Si}$ for interference by $\mathrm{Nd}, \mathrm{Er}$, and to $\mathrm{Pb}$ for interference by $\mathrm{Y}, \mathrm{Th}$, and to Th for interference by $\mathrm{Gd}, \mathrm{Y}$, and to Dy for interference by Th, and to Pr for interference by La, and to Er for interference by $\mathrm{Nd}$, and to $\mathrm{Nd}$ for interference by $\mathrm{Pb}, \mathrm{Ce}$, Th, and to $\mathrm{Gd}$ for interference by $\mathrm{Nd}, \mathrm{Ce}, \mathrm{La}$, and to $\mathrm{Sm}$ for interference by $\mathrm{Ce}$, and to $\mathrm{Yb}$ for interference by $\mathrm{Dy}$, and to $\mathrm{La}$ for interference by $\mathrm{Nd}$. Analytical results of analyzed monazite are found in Table 1.

\section{2- SHRIMP U-Th-Pb DATING}

$\mathrm{U}-\mathrm{Th}-\mathrm{Pb}$ isotope analyses of sectioned monazites were carried out on the SHRIMP II at Curtin University of Technology following the procedures described by Compston et al. (1986), Williams and Claesson (1987) and Smith et al. (1998). After detailed phothografy and BSE (backscatterd) imaging, the disc was cleaned and coated with high-purity gold. BSE images were acquired at CMCA (Center of de Microscopy, Characterization and Microanalysis). Monazite grains from two gold deposits, Trairão and Chumbo Grosso, were analyzed with $\sim 0.5 \mathrm{nA} \mathrm{O} \mathrm{O}_{2}^{-}$ 
prymary beans and $10 \mu \mathrm{m}$ spots. Analytical and data reduction methods for monazite are described by Rasmussen et al (2001).

Ages were calculated using U decay constants from Jaffey et al (1971), analytical uncertainties given in the table 2 are show in plots with 2 sigma. Plots were prepared using ISOPLOT 2.49 (Ludwig, 2001), after statistical analysis, the data were used in the construction of the concordia diagram.

\section{4- RESULTS}

Samples of hydrothermal monazite from Trairão (TR-20T) and Chumbo Grosso (TR-18S) deposits from the matrix of ore Quartz breccia rich in magnetite and Sericitic breccia, respectively, were analysed to constrain the age of ore formation. In the figure 05 are showed anhedric monazites with small size (10 to $30 \mu \mathrm{m})$ which they are commonly associated with barite and sulfides as pyrite and chalcopyrite. Similar features can be found in the figure 06. In figure 6 $\mathrm{B}$, we observe the agglomerate of monazite, whereas in the figure $6-\mathrm{D}$, is shown a monazite with thrre analysed spots of $\sim 10 \mu \mathrm{m}$.

In this paper, we document the chemical composition of hydrothermal monazites of gold ore paragenesis, which formed when the host biotite syenogranite was intensely altered by hydrothermal fluids. Using quantitative electron microprobe analysis, we discuss the element redistribution that occurred in conjunction with monazite formation. A total of 10 spots were analysed in 8 grains of monazite.

The chemical analyse results for 9 crystals of monazite, from the ore-bearing breccias of Trairão and Chumbo Grosso deposits, are presented in Table 01. The REE pattern normalized for Chondrites (Nakamura, 1974) shows high contents of La and depletion of Er. (Figure 07)

Monazites show positive correlation between $\mathrm{La}, \mathrm{Ce}, \mathrm{Eu}$ and enrichment of LREE over HREE. The values of $\mathrm{P}_{2} \mathrm{O}_{5}$ ranging from 28 to $30(\mathrm{ppm})$ and higher Ce concentrations (29.7 to $32.5 \%$ ) than $\mathrm{La}$ (15.3 to $17.6 \%$ ) defined as Ce-monazite, support the hypothesis that the low REE minerals (monazite) are part of the ore paragenesis and, therefore are suitable for dating mineralization. 

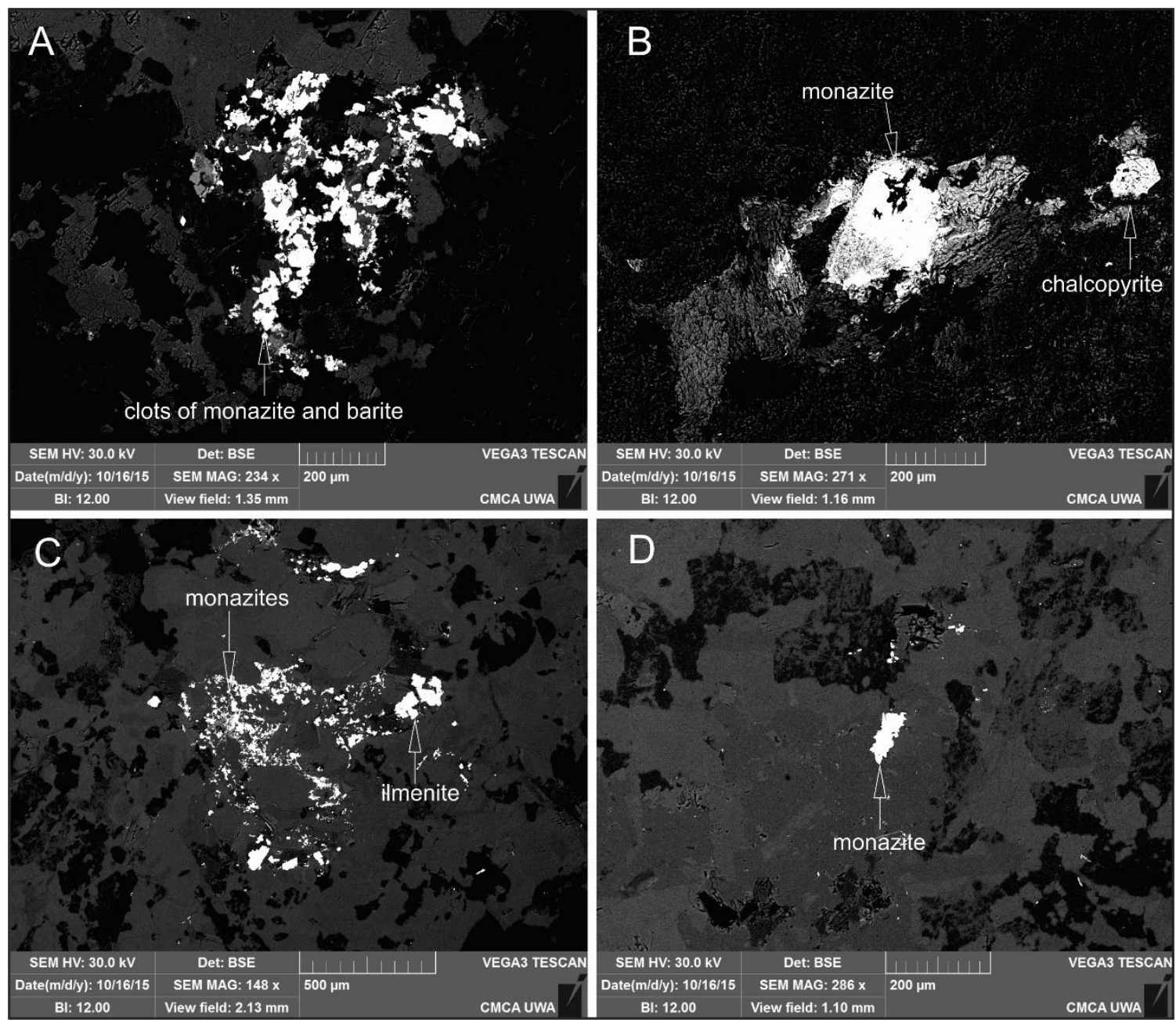

V Figure 05 - Representative backscattered electron images of thin section, sample TR-20T and TR-18S, showing monazite in apparent textural equilibrium with other minerals as barite, chalcopyrite and ilmenite, in the matrix of ore-bearing zone of Trairão and Chumbo Grosso deposit.

The mineralized breccia monazites also contain $\mathrm{Y}_{2} \mathrm{O}_{3}(0.4-1.7 \%), \mathrm{Pr}_{2} \mathrm{O}_{3}(3.4 \%), \mathrm{Nd}_{2} \mathrm{O}_{3}$ $(11.1-12.4 \%)$ and $\mathrm{Sm}_{2} \mathrm{O}_{3}(1.7-2.3 \%)$. The positive correlation between these elements suggests a common metasomatic origin. The occurrence of barite, galena, sphalerite, uraninite, monazite, xenotime as inclusion in chalcopyrite and gangue minerals in the mineralized breccia accounts for the high $\mathrm{La}, \mathrm{Ce}, \mathrm{Nd}, \mathrm{Eu}, \mathrm{Sm}, \mathrm{Pr}$ and $\mathrm{P}$.

The analyzed monazites have total values between 95.4 and $98.6 \%$, which can be attributed to the presence of unanalyzed elements such as $\mathrm{Fe}, \mathrm{F}, \mathrm{Al}$ and $\mathrm{Pb}$ radiogenic, or possible metamictization processes occurring in monazite (Kucha 1980, Förster 1998). 


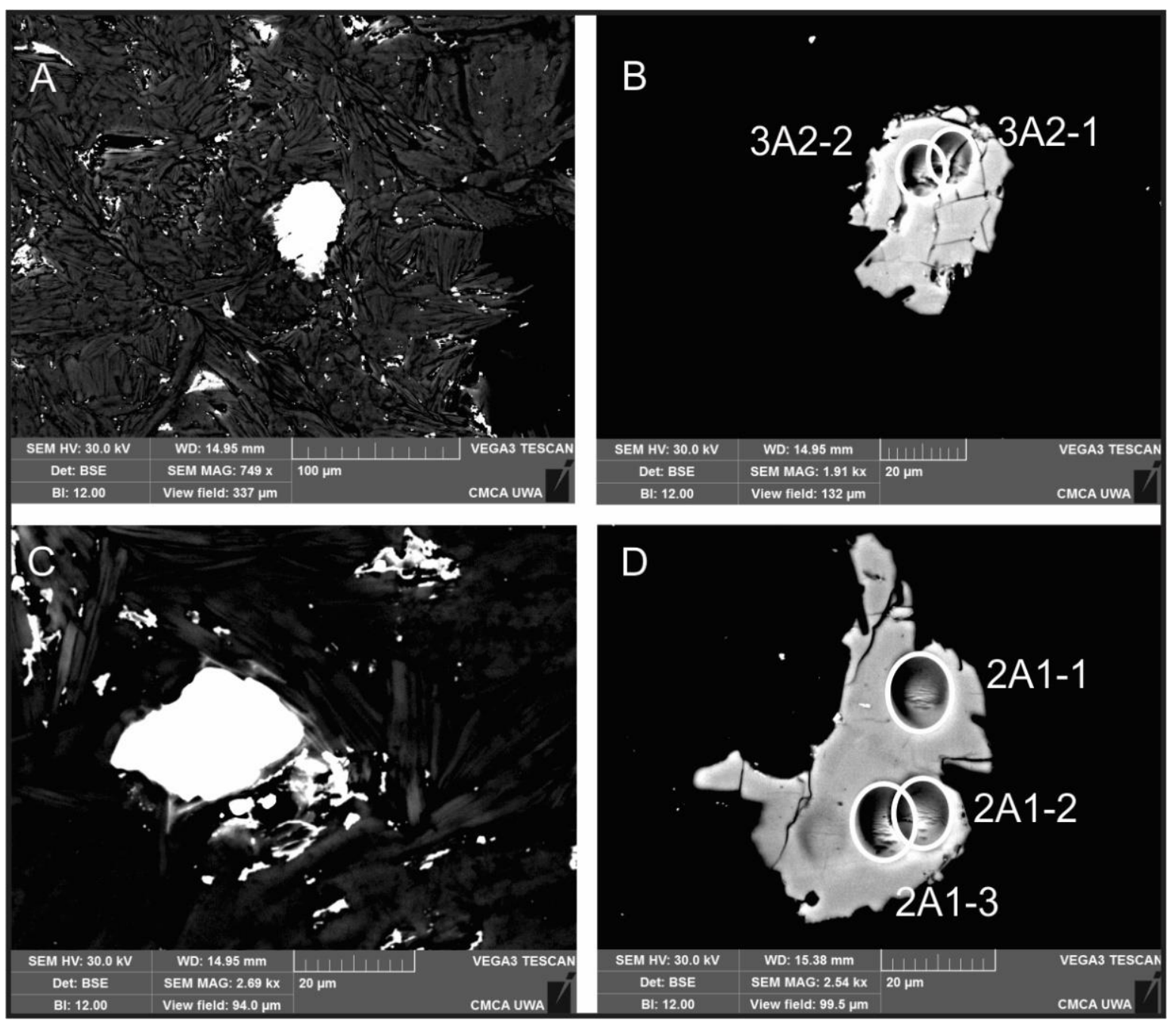

V Figure 06 - Scanning electron microscope backscattered electron (BSE) photomicrographs imagens of sample TR-20T and TR-18, of cross-sections of representative monazites. SHRIMP analytical areas are indicated by circles and are approximately $10 \mu \mathrm{m}$ in diameter.

The formation of monazite under hydrothermal fluid allowable action makes the possibility that at least part of their chemical composition, mainly concerning the U-Th- $\mathrm{Pb}$, is rebalanced after crystallization (Poitrasson et al. 1996). However, studies show that in the case of monazite generated during hydrothermal activity, the chemical imbalance between $\mathrm{U}-\mathrm{Th}-\mathrm{Pb}$, product of fluid-mineral interaction is minimal and occurs mainly along the fracture zones and mineral cleavage (Teufel \& Heinrich, 1997, Braun et al. 1998, Poitrasson et al. 2000). Thus, for chemical and SHRIMP II monazite analysis, we tried to avoid regions of the crystals with fractures and / or cavities (Figure 6). 


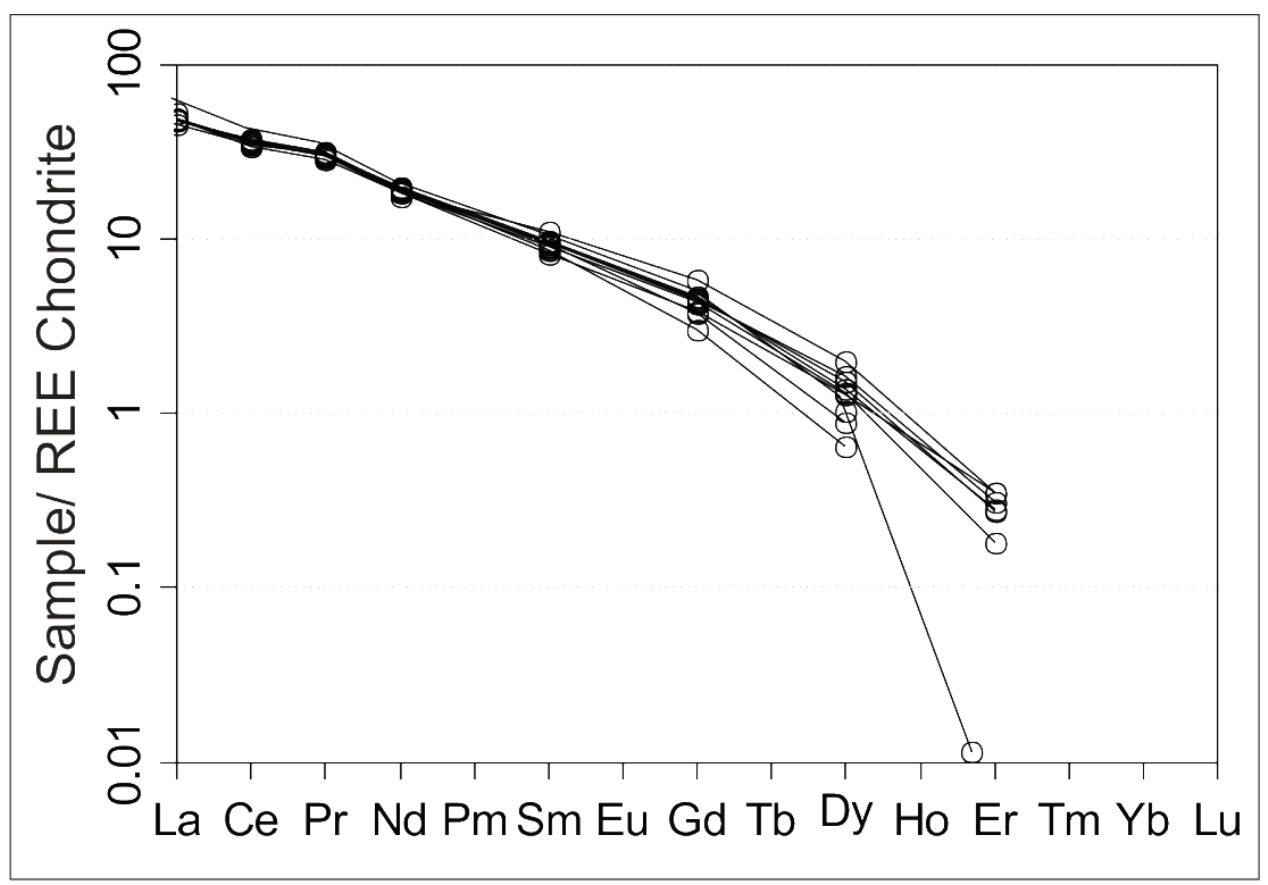

V Figure 07 - Representative rare earth element (REE) distribution patterns for monazites from mineralized zones. Chondrite values are from Nakamura (1974).

Two samples of monazite were dated, one of the Trairão and other of Chumbo Grosso gold deposits. .4 monazite grains or agglomerates have been dated by the SHRIMP method, which yielded six individual ages. Four individualconcordant analyses yielded a concordant age of Most of the U-Pb data fall in concordant clusters that provided the age of $1812 \pm 18 \mathrm{Ma}$ (2 sigma, MSDW = 2.5) (Figure 09). The two other analyses present larger errors and are discordant, so that they are not included for age calculation. The monazites of Chumbo Grosso are also concordant and three spots yielded an age of $1825 \pm 27 \mathrm{Ma}(2$ sigma, MSDW $=0.20)$ (Figure 10)

The Matupá Granite, a biotite syenogranite which hosts the two Au mineralizations at the Trairão region, has been dated by SHRIMP U-Pb zircon method. Eleven zircon grains have been analyzed and provided a concordant age of $1851 \pm 12 \mathrm{Ma}(2$ sigma, MSWD $=2.4)$ (Figure 11). 


\begin{tabular}{|c|c|c|c|c|c|c|c|c|c|}
\hline SAMPLE & $\begin{array}{l}\text { TR - 20T } \\
\text { 15-2A_001 }\end{array}$ & $\begin{array}{l}\text { TR - 20T } \\
\text { 15-2A_002 }\end{array}$ & $\begin{array}{l}\text { TR - 20T } \\
\text { 15-2A_003 }\end{array}$ & $\begin{array}{l}\text { TR -18S } \\
\text { 15-13C_001 }\end{array}$ & $\begin{array}{l}\text { TR -18S } \\
\text { 15-13B_001 }\end{array}$ & \begin{tabular}{|l} 
TR -18S \\
15-13B_002
\end{tabular} & $\begin{array}{l}\text { TR -18S } \\
\text { 15-13A_001 }\end{array}$ & $\begin{array}{l}\text { TR -18S } \\
\text { 15-13A_002 }\end{array}$ & $\begin{array}{l}\text { TR -18S } \\
\text { 15-13A_003 }\end{array}$ \\
\hline $\mathrm{SiO}_{2}$ & 0.0 & 0.1 & 0.0 & 0.9 & 0.2 & 0.3 & 0.1 & 0.1 & 0.3 \\
\hline $\mathrm{Y}_{2} \mathrm{O}_{3}$ & 1.6 & 1.6 & 1.7 & 0.7 & 1.4 & 1.0 & 1.6 & 0.5 & 0.4 \\
\hline $\mathrm{La}_{2} \mathrm{O}_{3}$ & 16.1 & 16.5 & 15.3 & 17.6 & 16.4 & 16.4 & 16.5 & 16.4 & 16.1 \\
\hline $\mathrm{Ce}_{2} \mathrm{O}_{3}$ & 31.3 & 31.5 & 30.6 & 31.2 & 31.2 & 30.8 & 29.7 & 32.2 & 32.5 \\
\hline $\mathrm{Pr}_{2} \mathrm{O}_{3}$ & 3.4 & 3.4 & 3.5 & 3.3 & 3.4 & 3.4 & 3.2 & 3.6 & 3.5 \\
\hline $\mathrm{Nd}_{2} \mathrm{O}_{3}$ & 12.1 & 11.6 & 12.4 & $\mid 11.1$ & 12.0 & 11.8 & 11.6 & 12.4 & 12.2 \\
\hline $\mathrm{Sm}_{2} \mathrm{O}_{3}$ & 2.0 & 1.7 & 2.0 & 1.8 & 1.9 & 1.8 & 2.3 & 1.9 & 1.8 \\
\hline $\mathrm{Gd}_{2} \mathrm{O}_{3}$ & 1.2 & 1.1 & 1.3 & 1.2 & 1.3 & 1.2 & 1.6 & 1.0 & 0.8 \\
\hline $\mathrm{Dy}_{2} \mathrm{O}_{3}$ & 0.6 & 0.4 & 0.5 & 0.4 & 0.5 & 0.5 & 0.7 & 0.3 & 0.2 \\
\hline $\mathrm{Er}_{2} \mathrm{O}_{3}$ & 0.1 & 0.1 & 0.1 & 0.0 & 0.1 & 0.0 & 0.1 & 0.0 & 0.0 \\
\hline $\mathrm{Yb}_{2} \mathrm{O}_{3}$ & 0.0 & 0.0 & 0.0 & 0.0 & 0.0 & 0.0 & 0.0 & 0.0 & 0.0 \\
\hline $\mathrm{CaO}$ & 0.2 & 0.2 & 0.3 & 0.3 & 0.3 & 0.3 & 0.2 & 0.1 & 0.2 \\
\hline $\mathrm{P}_{2} \mathrm{O}_{5}$ & 30.0 & 30.2 & 29.9 & 28.1 & 29.0 & 27.9 & 30.4 & 29.9 & 29.3 \\
\hline SUM & 98.6 & 98.4 & 97.6 & 96.6 & 97.7 & 95.4 & 98 & 98.4 & 97.3 \\
\hline
\end{tabular}

V Table 01 - Microprobe analyses in oxide formula from the grains of monazite, samples TR - 20T and TR - 18S, value are present in percentage. 


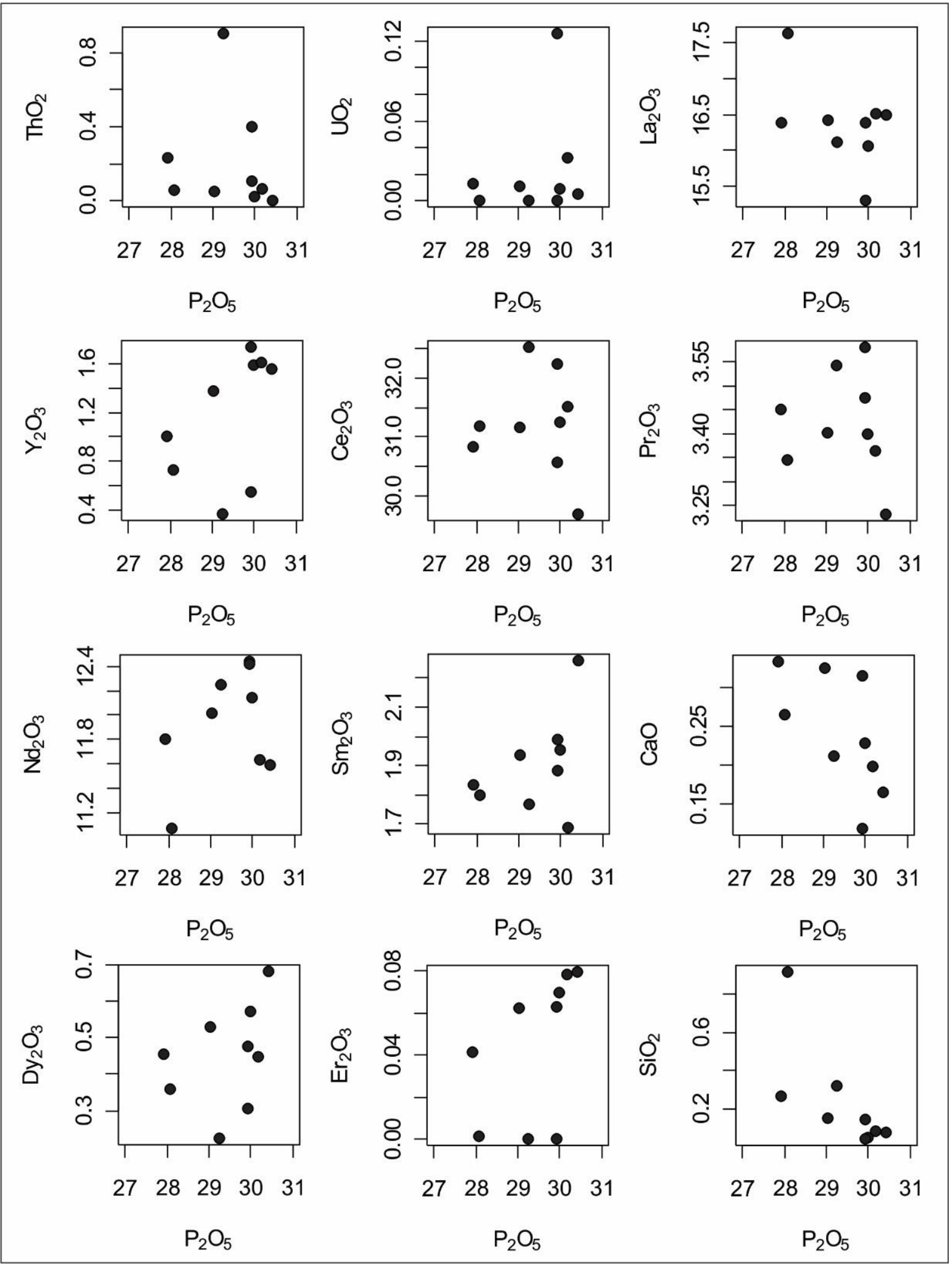

V Figure 08 - Bivariate diagrams for trace elements for monazites from mineralized zones. 


\section{1-U-Pb-Th RESULTS}

\begin{tabular}{|c|c|c|c|c|c|c|c|c|c|c|c|c|c|c|c|c|c|c|c|c|c|}
\hline $\mathrm{TR}-20 \mathrm{~T}$ & $\begin{array}{c}\text { 4-corr } \\
\% \text { com } \\
206\end{array}$ & $\begin{array}{c}\mathrm{U} \\
\mathrm{ppm}\end{array}$ & $\begin{array}{c}\text { Th } \\
\mathrm{ppm}\end{array}$ & $\begin{array}{l}\text { 4-corr } \\
\text { ppm } \\
206^{*}\end{array}$ & $\begin{array}{l}{ }^{232} \mathrm{Th} \\
{ }^{238} \mathrm{U}\end{array}$ & $\begin{array}{c}{ }^{204 \text { corr }} \\
{ }^{206} \mathrm{~Pb} \\
\rho^{238} \mathrm{U} \\
\text { Age }\end{array}$ & $\begin{array}{l}1 \mathrm{~s} \\
\text { err }\end{array}$ & $\begin{array}{c}204 \text { corr } \\
{ }^{207} \mathrm{~Pb} \\
{ }^{206} \mathrm{~Pb} \\
\text { Age }\end{array}$ & $\begin{array}{c}1 \mathrm{~s} \\
\text { err }\end{array}$ & $\begin{array}{c}204 \text { corr } \\
{ }^{208} \mathrm{~Pb} \\
{ }^{232} \mathrm{Th} \\
\text { Age }\end{array}$ & $\begin{array}{c}1 \mathrm{~s} \\
\text { err }\end{array}$ & $\begin{array}{c}\% \\
\text { Dis- } \\
\text { cor- } \\
\text { dant }\end{array}$ & $\begin{array}{l}\text { 4corr } \\
208^{*} \\
1232\end{array}$ & $\begin{array}{l}\% \\
\text { err }\end{array}$ & $\begin{array}{l}4 \text { corr } \\
207 * \\
1206^{*}\end{array}$ & $\begin{array}{l}\% \\
\text { err }\end{array}$ & $\begin{array}{l}4 \operatorname{corr} \\
207 * \\
/ 235\end{array}$ & $\begin{array}{c}\% \\
\text { err }\end{array}$ & $\begin{array}{c}4 \text { corr } \\
206^{*} \\
/ 238\end{array}$ & $\begin{array}{c}\% \\
\text { err }\end{array}$ & $\begin{array}{l}\text { err } \\
\text { corr }\end{array}$ \\
\hline 1512A.1-1 & 0.41 & 288 & 581 & 80 & 2 & 1808 & 32 & 1812 & 19 & 1864 & 28 & $\overline{0}$ & 0.0966 & 1.6 & 0.11079 & 1.0 & 4.9446 & 2.3 & 0.3237 & 2.1 & 0.894 \\
\hline 1512A.1-2 & 0.08 & 847 & 2275 & 244 & 2.8 & 1862 & 28 & 1790 & 17 & 1862 & 40 & -5 & 0.0965 & 2.3 & 0.10941 & 0.9 & 5.0508 & 2.0 & 0.3348 & 1.7 & 0.882 \\
\hline 1512A.1-3 & 0.19 & 1009 & 2044 & 287 & 2 & 1843 & 25 & 1805 & 27 & 1803 & 27 & -2 & 0.0933 & 1.6 & 0.11036 & 1.5 & 5.0362 & 2.1 & 0.3310 & 1.6 & 0.728 \\
\hline 1512C.1-1 & 3.84 & 136 & 1618 & 37 & 12.3 & 1766 & 30 & 1888 & 140 & 1865 & 37 & 7 & 0.0967 & 2.1 & 0.11552 & 7.8 & 5.0207 & 8.0 & 0.3152 & 1.9 & 0.239 \\
\hline 1512D.1-1 & 2.11 & 152 & 11236 & 42 & 76.2 & 1794 & 29 & 1794 & 61 & 1865 & 36 & $\mathbf{0}$ & 0.0967 & 2 & 0.10968 & 3.3 & 4.8532 & 3.8 & 0.3209 & 1.9 & 0.489 \\
\hline 1512E.1-1 & 1.9 & 633 & 61799 & 202 & 101 & 2039 & 49 & 1809 & 93 & 1987 & 27 & -15 & 0.1033 & 1.4 & 0.11061 & 5.1 & 5.673 & 5.8 & 0.3720 & 2.8 & 0.483 \\
\hline TR $-18 \mathrm{~S}$ & $\begin{array}{c}\text { 4-corr } \\
\% \text { com } \\
206\end{array}$ & $\begin{array}{c}\mathrm{ppm} \\
\mathrm{U}\end{array}$ & $\begin{array}{c}\text { ppm } \\
\text { Th }\end{array}$ & $\begin{array}{c}\text { 4-corr } \\
\text { ppm } \\
206^{*}\end{array}$ & $\begin{array}{l}{ }^{232} \mathrm{Th} \\
{ }^{238} \mathrm{U}\end{array}$ & $\begin{array}{c}204 \text { corr } \\
{ }^{206} \mathrm{~Pb} \\
{ }^{238} \mathrm{U} \\
\text { Age }\end{array}$ & $\begin{array}{l}1 \mathrm{~s} \\
\text { err }\end{array}$ & $\begin{array}{c}204 \text { corr } \\
{ }^{207} \mathrm{~Pb} \\
{ }^{206} \mathrm{~Pb} \\
\text { Age }\end{array}$ & $\begin{array}{l}1 \mathrm{~s} \\
\text { err }\end{array}$ & $\begin{array}{c}204 \text { corr } \\
{ }^{208} \mathrm{~Pb} \\
{ }^{232} \mathrm{Th} \\
\text { Age }\end{array}$ & $\begin{array}{c}1 \mathrm{~s} \\
\text { err }\end{array}$ & $\begin{array}{c}\% \\
\text { Dis- } \\
\text { cor- } \\
\text { dant }\end{array}$ & $\begin{array}{l}\text { 4corr } \\
208^{*} \\
/ 232\end{array}$ & $\begin{array}{l}\% \\
\text { err }\end{array}$ & $\begin{array}{l}4 \text { corr } \\
207^{*} \\
/ 206^{*}\end{array}$ & $\begin{array}{l}\% \\
\text { err }\end{array}$ & $\begin{array}{l}4 \text { corr } \\
207 * \\
/ 235\end{array}$ & $\begin{array}{c}\% \\
\text { err }\end{array}$ & $\begin{array}{l}4 \text { corr } \\
206^{*} \\
/ 238\end{array}$ & $\begin{array}{c}\% \\
\text { err }\end{array}$ & $\begin{array}{l}\text { err } \\
\text { corr }\end{array}$ \\
\hline 1513A.1-1 & 0.99 & 237 & 641 & 66 & 3 & 1816 & 32 & 1836 & 30 & 1828 & 28 & 1 & 0.0947 & 1.6 & 0.11226 & 1.7 & 5.0381 & 2.6 & 0.3255 & 2.0 & 0.774 \\
\hline 1513A.2-1 & 4.65 & 33 & 3180 & 9 & 101 & 1811 & 44 & 1889 & 147 & 1870 & 24 & 5 & 0.0969 & 1.4 & 0.11557 & 8.2 & 5.1681 & 8.6 & 0.3243 & 2.8 & 0.321 \\
\hline 1513A.2-2 & 5.3 & 64 & 7126 & 18 & 116 & 1841 & 43 & 1854 & 161 & 1863 & 24 & 1 & 0.0966 & 1.3 & 0.11334 & 8.9 & 5.165 & 9.3 & 0.3305 & 2.7 & 0.287 \\
\hline 1513B.1-1 & 3.03 & 241 & 1107 & 69 & 5 & 1847 & 29 & 1819 & 50 & 1789 & 27 & -2 & 0.0926 & 1.6 & 0.11118 & 2.8 & 5.0846 & 3.3 & 0.3317 & 1.8 & 0.542 \\
\hline 1513B.2-1 & 1.32 & 279 & 1121 & 78 & 4 & 1807 & 28 & 1818 & 40 & 1784 & 27 & 1 & 0.0923 & 1.6 & 0.11113 & 2.2 & 4.9584 & 2.8 & 0.3236 & 1.8 & 0.628 \\
\hline
\end{tabular}

V Table 02 - SHRIMP II, U-Th-Pb isotopic data for monazites from, samples TR - 20T (Trairão Deposit) and TR - 18S (Chumbo Grosso Deposit). 


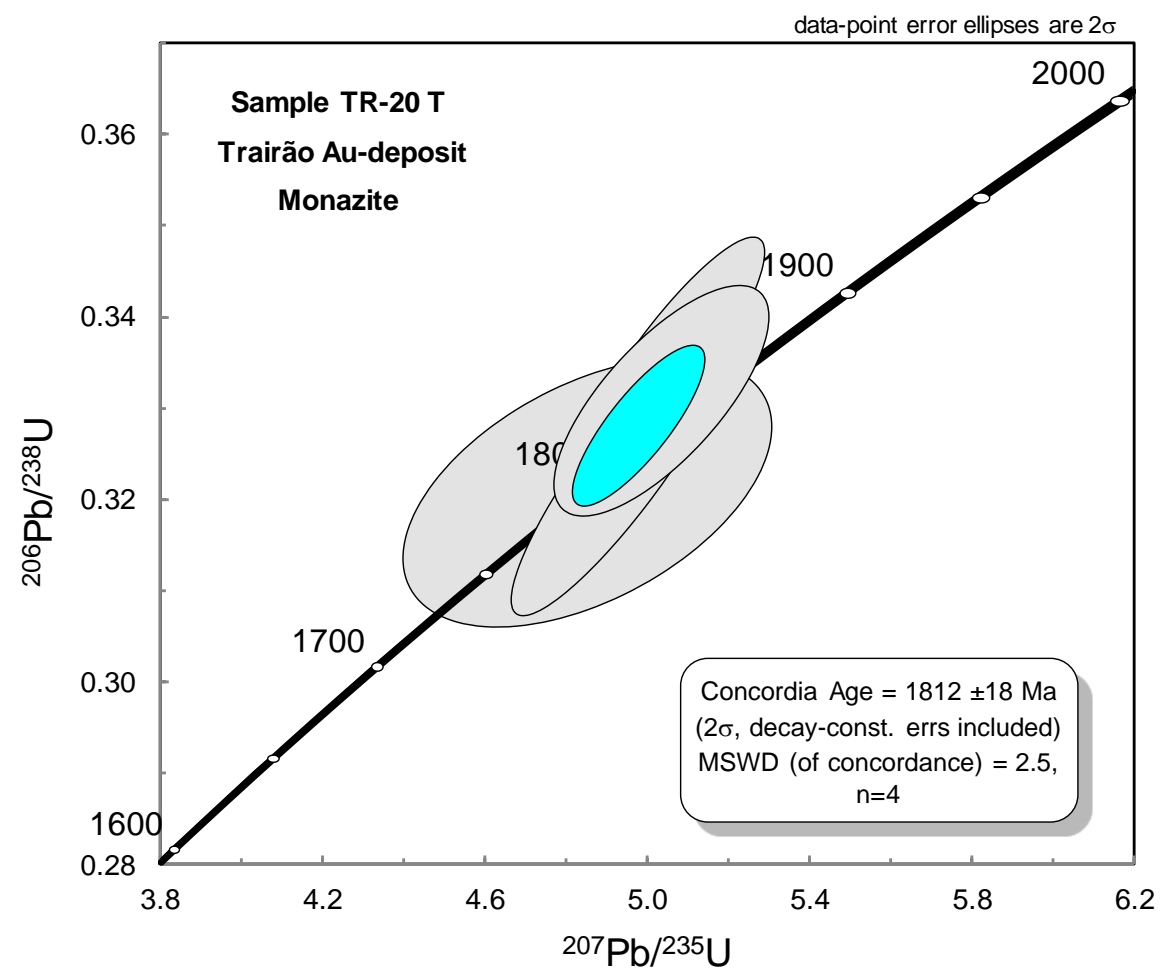

V Figure 09 - Concordia diagram of SHRIMP II data for monazite from the Aumineralized veins at the Trairão deposit. Shown in the inset, is $1812 \pm 18 \mathrm{Ma}$ $(\mathrm{MSWD}=2.5)$.

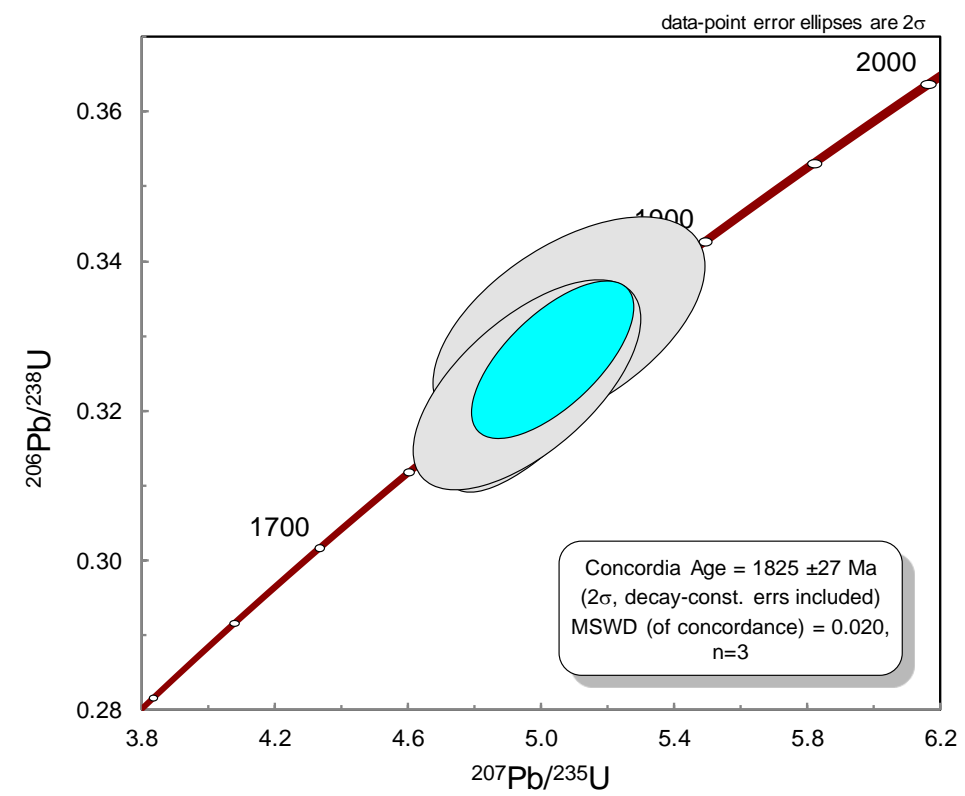

V Figure 10 - Concordia diagram of SHRIMP II data for magmatic monazite from the ore rock. Shown in the inset, is $1825 \pm 27 \mathrm{Ma}(\mathrm{MSWD}=0.2$ ). 


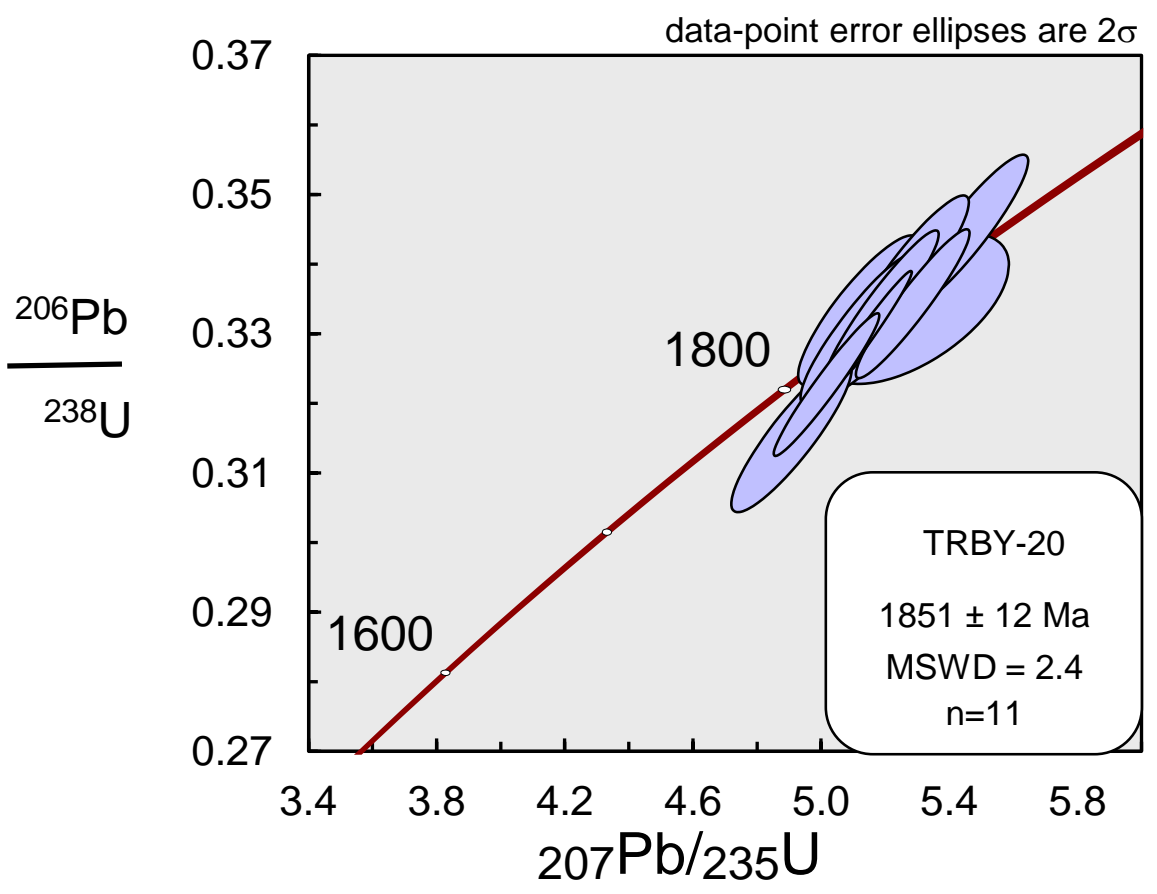

V Figure 11 - Concordia diagram of SHRIMP II data for magmatic zircon sample TR-20. Shown in the inset, is $1851 \pm 12 \mathrm{Ma}$ interpreted as the crystallization age of the granitic massive (MSWD $=2.4$ ).

\begin{tabular}{|c|c|c|c|c|c|c|}
\hline SAMPLE & ROCK TYPE & COORDINATES $*$ & $\operatorname{AGE}(\mathrm{Ma})$ & $\begin{array}{l}\mathrm{SiO} 2 \\
(\% \mathrm{wt}) \\
\end{array}$ & $\begin{array}{c}\text { ANALYZED } \\
\text { MINERAL }\end{array}$ & $\begin{array}{c}\mathrm{Zr} \\
(\mathrm{ppm})\end{array}$ \\
\hline TR-20T & $\begin{array}{c}\text { Au rich } \\
\text { quartz vein - } \\
\text { Hydrotermal rock }\end{array}$ & $\begin{array}{l}09^{\circ} 48^{\prime} 25^{\prime \prime} \\
55^{\circ} 34^{\prime} 47^{\prime \prime} \\
\end{array}$ & $\begin{array}{c}1814 \pm 9.4 \mathrm{Ma} \\
\text { Minimum age } \\
\text { of ore formation }\end{array}$ & $75 \%$ & monazite & 76 \\
\hline TR-18S & $\begin{array}{c}\text { Au - Magnetite } \\
\text { rich breccia - } \\
\text { Hydrotermal rock }\end{array}$ & $\begin{array}{l}09^{\circ} 47^{\prime} 37^{\prime \prime} \\
55^{\circ} 40^{\prime} 43^{\prime \prime}\end{array}$ & $\begin{array}{c}1825 \pm 13 \mathrm{Ma} \\
\text { Minimum age } \\
\text { of ore formation }\end{array}$ & $72 \%$ & monazite & 72 \\
\hline TR-20 & $\begin{array}{c}\text { Biotite } \\
\text { syenogranite - } \\
\text { Host rock } \\
\end{array}$ & $\begin{array}{l}09^{\circ} 48^{\prime} 25^{\prime \prime} \\
55^{\circ} 35^{\prime} 11^{\prime \prime} \\
\end{array}$ & $\begin{array}{c}1851 \pm 12 \mathrm{Ma} \\
\text { Magmatic } \\
\text { crystallization } \\
\end{array}$ & $76 \%$ & zircon & 75 \\
\hline
\end{tabular}

V Table 03 - Description of samples from Trairão and Chumbo Grosso Deposits. Minerals selected for SHRIMP II analyses.

* Coordinates are referenced to SAD 69 (South American Datum) zone 22 Southern Hemisphere. 


\section{5-DISCUSSIONS}

The present data demonstrate that hydrothermal monazites ages offer great potential in helping our understanding of hydrothermal mineralization in this part of Amazonian Craton. Souza et al. (2005) dated already hydrothermal monazites in the Sn-bearing Bom Futuro at $997 \pm 48$ Ma. Monazites has been shown to be useful for dating magmatic and high-temperature metamorphic events (Parrish, 1990), but this mineral can be available to dating hydrothermal events, once proven your hydrothermal origin using quantitative electron microprobe analysis. Usually, the Electron Microprobe Analyses (EMPA) allow determine the age of monazite crystals with high spatial resolution (up to $1 \mu \mathrm{m}$ of crystal diameter), but it is most used to date monazite with high amount of Th and U (Montel et al., 1996, Cocherie et al., 2005). However, this method has some limitation to provide precise age to hydrothermal monazite grains with low Th and U amounts, where the monazite crystals display complex, small-scale growth zoning. By using high resolution sensitive ion microprobe (SHRIMP) combined with detailed petrographic, mineral chemistry is it possible to determine an age of lowgrade metamorphism or hydrothermal event with the high precision in situ geochronology (e.g. Ramussen et al., 2006).

The Au-mineralization quartz vein of Trairão deposit is hosted in the Matupá granite. The ore paragenesis of the Trairão deposit, which is characterized by pyrite + barite + chalcopyrite + monazite, has hydrothermal monazite crystals that contain significant amounts of F, P, La and Ce. These crystals have also variable Th $(0.06$ to $6.18 \%)$ and $\mathrm{U}$ (152 to $1009 \mathrm{ppm}$ ) contents, clearly different from typical magmatic and metamorphic samples (Chang et al., 1996). The hydrothermal monazites of Trairão deposits are therefore characterized as monazite richer in Ce than La. The positive correlation between these elements suggests a common metasomatic origin. The occurrence of barite, galena, sphalerite, uraninite, monazite, xenotime as inclusion in chalcopyrite and gangue minerals in the mineralized breccia accounts for the high La, $\mathrm{Nd}, \mathrm{Sm}$ and Pr. The monazites of Chumbo Grosso gold deposit have similar textural and chemical characteristics to those monazites of the Trairão gold deposit, containing lower Th (0.064 to 0.75$)$ and $U$ (33 to $279 \mathrm{ppm})$ content.

The U-Th-Pb SHRIMP were carried out in different monazites crystals and aggregate of hydrothermal and magmatic origin in the Trairão region. The SHRIMP II zircon age 
of the host rocks, the Matupá Granite, exposed in the Trairão deposit, it is dated at 1851 \pm 12 (Table 3, Fig.11) based on the U-Pb concordia age of 11 zircon grains.

Samples of hydrothermal monazite (Trairão TR-20T and Chumbo Grosso Deposits TR-18S) from the matrix of ore Quartz breccia rich in magnetite and sericite breccias with anhedric habit and grain size from 10 to $30 \mu \mathrm{m}$ (Fig. 3) yielded an ${ }^{206} \mathrm{~Pb} /{ }^{238} \mathrm{U}$ ages of $1812 \pm 18 \mathrm{Ma}$ and $1825 \pm 27 \mathrm{Ma}$, where the monazites are commonly associated with barite and sulphites as pyrite and chalcopyrite. We conclude that hydrothermal monazites of mineralized breccia, based on mineralogical, geochemical and geochronologic data herein presented, from the Trairão and Chumbo Grosso ore deposits indicated an epigenetic hydrothermal deposit with magmatic origin.

Alta Floresta Gold Province is a continental magmatic arc with several flare-up (high volume of magmatism) and steady-state (quiescence or low volume of magmatism) as presented by several authors (eg.: Moura \& Botelho, 2002, Bettencourt et al., 2016). They are recognized several magmatig events, as follow: made by the occurrence of several tapajônicos magmatic arcs (in sense of Bettencourt et al., 2016) with the following chronology (Fig. 11): Arc 1 - Cuiú-Cuiú Arc: 2.0 Ga - 1.98 Ga; Arc 2 Creporizão - Nhandu Arc: 1.96 to $1.94 \mathrm{Ga}$; Arc 3 - Parauari -Matupá Arc: 1.88 to 1.84 $\mathrm{Ga}$, Arc 4 - Juruena Arc 1.82-1.79 Ga, and Colider Arc (volcano-plutono rocks and associated rocks) 1.79-1.78 Ga. The later magmatic pulse of this region, the Teles Pires magmatism and related rocks, is marked by expressive volcanic and plutonic activity of A-type acid rocks formed between 1.78-1.75 Ga, described as post-collisional magma type.

Hydrothermal gold and gold-base metal deposits in the AFGP mainly consist of quartz veins and hydrothermal breccias associated with the wideaspread magmatic activity and are structural controlled. Hydrothermal events are responsible for late fluids that percolated the granites of different ages. Based on Re-Os dating, Assis (2015) establish that $\mathrm{Au}$ disseminated deposits formed at 1.787 $\pm 6.2 \mathrm{Ma}$ (Luizão), $1.787 \pm 5.5 \mathrm{Ma}$ (Pé Quente), and $1.786 \pm 5 \mathrm{Ma}$, whereas the same author working with Franscico deposit, a gold-base metal similar to the Trairão deposit, establish that ${ }^{40} \mathrm{Ar} /{ }^{39} \mathrm{Ar}$ plateau ages from the sericitic alteration halo yielded ages from $1.779 \pm 6.6$ Ma and $1.777 \pm 6.4$. He concludes that the gold and gold-base metal mineralization of 
the studied deposits occurred closely associated with 1.78-1.75 Ga post-collisional magmatism of Teles Pires and related rocks.

The obtained age gold $(\mathrm{Cu})$ mineralization in the present study points to a mineralization age associated with period of the Juruena Arc magmatic activity, since the obtained monazite ages are dated at 1.812-1.825 Ga. Take into account the age of Matupá Granite, the host rocks of the gold mineralization, formed at $1850 \mathrm{Ma}$, there is not presence of younger magma magmatism at the Trairão region. So the processes occurred by fluid percolation and mineral precipitation, evidenced by the presence of Au mineral deposits in mineralized hydrothermal breccias during 1.81-1.82 Ga which cut the host Matupá Granite dated at $1.85 \mathrm{Ga}$. Therefore, gold mineralization is younger than host rocks and it is ca. 25 - 40 millions of years younger than host rocks, the Matupá Granite.

\section{6- CONCLUSIONS}

The gold deposits at the Trairão region are associated with breccias and quartz veins hosted in granites evolved in a continental magmatic arc. The age of these arcs (Rocha, et al. 2016, submitted) range from 1960 to $1946 \mathrm{Ma}$, (Nhandu Intrusive Suite) and 1878-1837 Ma (Matupá Intrusive Suite). These Au-bearing breccias exhibit a fine grained matrix of subhedral magnetite cemented by silica together with sulfides (pyrite, galena, chalcopyrite, sphalerite), monazite, barite rarely uraninite and xenotime. Based on the textural and chemical composition of the monazites with high content of $\mathrm{Ce}$, $\mathrm{P}_{2} \mathrm{O}_{5}$, and $\mathrm{La}$, they are classified as hydrothermal Ce-monazites which they are intrinsic related to hydrothermal breccias and veins of the Trairão and Chumbo Grosso gold deposits.

The U-Th-Pb in hydrothermal monazite yielded an age of $1812 \pm 18 \mathrm{Ma}$ and $1825 \pm$ $27 \mathrm{Ma}$, so that the Au-mineralization formed ca. 25-40 millions of years younger than the host Matupá Granite, dated at $1851 \pm 12 \mathrm{Ma}$. Many intrusions in the AFGP shows the same age: Paranaíta Suite, Cristalino Granite, Guarantã Granite and here they are correlated this granite with the Juruena Intrusive Suite, which they are part of one important mineralization process in Alto Floresta Gold Province.

Tese de Doutorado - Mara Luiza Barros Pita Rocha 
The styles of hydrothermal alteration, mineralogy, as well as published fluid inclusion data support a classification as a member of granitic rocks genetically related to disseminated mineralization of gold and gold-copper, similar to hypothesis proposed by Moura (2002) to Serrinha deposit (Matupá Intrusive Suite).

\section{7 - ACKNOWLEDGMENTS}

We are gratefully acknowledged to the Postgraduate Program of Geosciences of Universidade de Brasília (UnB), Curtin University of Technology and Western Australia University for support to this research. The authors thank especially to CNPq for PhD scholarship.

\section{8 - REFERENCES}

Almeida F.F.M.; Hasui Y.; Brito Neves B.B.; Fuck R.A. 1981. Brazilian structural provinces: an introduction. Earth Sci. Rev. 17, 1-29.

Amaral, G.; 1974. Geologia Pré-Cambriana da Região Amazônica. Universidade de São Paulo, Tese de Livre Docência, São Paulo, 212 pp.

Assis, R.R.; Xavier R.P.; Paes de Barros A.J.; Barbuena D.; Miguel-Jr E.; 2012. Contexto geológico e litogeoquímica das unidades plutônicas-vulcânicas da região de União do Norte, setor leste da Província Aurífera de Alta Floresta (MT). Rev. Bras. Geoc., 42:130-161.

Assis, R.R.; 2015. Depósitos Auríferos Associados ao Magmatismo Félsico da Província de Alta Floresta (MT), Craton Amazônico: Litogeoquímica, Idade das Mineralizações e Fonte dos Fluidos. Tese de doutorado. Departamento de Geologia e Recursos Naturais. Universidade de Campinas. SP.

Berger, A.; Gnos, E.; Schreurs, G.; Fernàndez, A.M.; Rakotondrazafy, M.; 2006, Late Neoproterozoic, Ordovician and Carboniferous events recorded in monazites from southem-central Madagascar. Precambrian Research 144, 278-296.

Braun, I.; Montel, J-M.; Nicollet, C.; 1998. Electron microprobe dating of monazites from high-grade gneisses and pegmatites of the Kerala Khondalite Belt, southern India. Chem. Geol., 146:65-85.

Bini, E. G.; Barros, M. A. S.; Pierosan, R.; Santos, J. O. S.; 2015. Petrography and geochronology of felsic volcanic rocks at the eastern portion of Serra do Cachimbo, south-central Amazonian Craton, Brazil. In: VI Simpósio de Vulcanismo e Ambientes Associados, 2015, São Paulo. Boletim de Resumos, v. 1. p. 67.

Candela, P.A.; 1997. A review of shallow, ore-related granites: textures, volatiles, and metals. J. Petrol.38:1619-1633. 
Chang, L.L.Y.; Howie, R.A.; Zussman, J.; 1996, Rock-forming minerals. Volume 5B: Nonsilicates: Sulphates, carbonates, phosphates and halides: Harlow, UK, Longman Group, 383 p.

Cherniak, D.J.; Watson, E.B.; Grove, M.; Harrison, T.M.; 2004. Pb diffusion in monazite: a combined RBS/SIMS study. Geoch. Cosmoch Act, 68, 829-840.

Compston, W.; Williams, I.S.; Campbell, I.H.; Gresham, J.J.; 1986. Zircon xenocrysts from the Kambalda volcanics: Age constraints and direct evidence for the older continental crust below the Kambalda-Norseman greenstones: Earth and Planetary Science Letters, v. 76, p. 299-311.

Cordani, U. G.; Teixeira W.; 2007. Proterozoic Accretionary belts in the Amazonian Cráton. Geological Society of America, v.200, p.297-320.

Cordani U.G.; Tassinari C.C.G.; Teixeira W.; Basei M.A.S. \& Kawasita K.; 1979. Evolução Tectônica da Amazônia com base nos dados geocronológicos. In: Congresso Chileno, 2, Chile. Atas, p. 137-148.

Dardene. M.A; Schobbenhaus, C.; 2001. Metalogênese do Brasil. Brasília, Ed Universidade de Brasília, 392p.

Duarte, T. B.; 2015. Geologia, Geoquímica e Geocronologia do Domínio Vulcânico do Arco Magmático Juruena, SW do Cráton Amazônico: Implicações Geotectônicas. Dissertação de Mestrado; Instituto de Geociências, Universidade Estadual de Campinas.

Fernandes, C.M.D.; Juliani, C.; Monteiro, L.V.S.; Lagler, B.; Misas, C.M.E.; 2011. High-K calc-alkaline to A-type fissure-controlled Vulcano-plutonism of the São Félix do Xingu region, Amazonian Craton, Brazil: Exclusively crustal sources or only mixed $\mathrm{Nd}$ model ages? Journal of South American Earth Sciences.

Förster, H.J.; 1998. The chemical composition of REE-Y-Th-U rich accessory minerals in peraluminous granite of the Erzgebirge- Fichtelgebirge region, Germany, Part I: the monazite - $(\mathrm{Ce})$ - brabanite solid solution series. Amer. Mineral., 83:259-272.

Jaffey, A.H.,; Flynn, K.F.; Glendenin, L.E.; Bentley, W.C.; Essling, A.M.; 1971. Precision measurement of half lives and specific activities of 235U and 238U: Physics Reviews, v. C4, p. 1889-1906.

Juliani C.; Carneiro C.C.; Carreiro-Araújo S.A.; Fernandes C.M.D.; Monteiro L.V.S.; Crósta A.P.; 2013. Estruturação dos arcos magmáticos paleoproterozóicos na porção sul do Cráton Amazônico: implicações geotectônicas e metalogenéticas. In: 13 Simpósio de Geologia da Amazônia, SBG - Núcleo Norte, Belém. (CD-ROM).

Juliani, C.; Vasquez M.L.; Klein E.L.; Villas R.N.N.; Echeverri-Misas C.M.; Santiago E.S.B.; Monteiro, L.V.S.; Carneiro C. de C.; Fernandes C.M.D.; 2014. Metalogênese da Província Tapajós. In: Silva, M.G., Rocha Neto, M.B., Jost, H., Kuyumjian, R.M. (Org.) Metalogênese das Províncias Tectônicas Brasileiras, Programa Geologia do Brasil, Recursos Minerais, Serviço Geológico do Brasil, CPRM, p. 229-263.

Krenn, E.; Putz, H.; Finger, F.; Paar, W. H.; 2011. Sulfur-rich monazite with high common $\mathrm{Pb}$ in ore-bearing schists from the Schellgaden mining district, Tauern Window, Eastern Alps. Miner Petrol 102:51-62. 
Kucha, H.; 1980. Continuity in the monazite-hyuttonite series. Mineral. Mag., 43:10311034.

Ludwig, K.R.; 2001. Using Isoplot/Ex. A geochronological toolkit for Microsoft Excel. Berkeley Geochronology Center, Special Publications No.1, Berkeley, USA.

Meldrum, A.; Boatner L. A.; Weber W. J.; Ewing R. C.; 1998. Radiation damage in zircon and monazite. Geochim. Cosmochim. Acta 62, 2509-2520

Meldrum, A.; Boatner, L.A.; Zinkle, S.J.; Wang, S.-X.; Wang, L.-M.; and Ewing, R.C.; 1999. Effects of dose rate and temperature on the crystalline-to-metamict transformation in the $\mathrm{ABO}_{4}$ orthosilicates. Canadian Mineralogist, 37, 207-221.

Meldrum, A.; Boatner, L.A.; Ewing, R.C.; 2000. A comparison of radiation effects in crystalline ABO4-type phosphates and silicates. Mineralogical Magazine, 64, 185-194.

Miguel Jr, E.; 2011. Controle Estrutural das mineralizações auríferas e idades U-Pb das rochas encaixantes ao longo do Lineamento Peru-Trairão: Província Aurífera de Alta Floresta, Mato Grosso. Qualificação - Dissertação de Mestrado, Instituto de Geociências, Universidade Estadual de Campinas, 41p.

Moura, M.A.; Botelho, N.F.; 2002. Petrologia do magmatismo associado à mineralização do tipo ouro pórfiro a província aurífera Juruena -Teles Pires (MT). Rev. Bras. Geoc., 32:377-386.

Nakamura, N. 1974. Determination of REE, Ba, Fe, $\mathrm{Mg}, \mathrm{Na}$ and $\mathrm{K}$ in carbonaceous and ordinary chondrites. Geoch. Cosmoch Acta, 38(5):757-775.

Nasdala, L.; Finger, F.; Kinny, P.; 1999. Can monazite become metamict? Eur J. Mineral 11, Beic 1:164

Neder, R. D.; Figueiredo, B. R.; Beaudry, C., Collins, C.; Leite, J. A. D.; 2000. The Expedito Massive Sulfide Deposit - Mato Grosso: Revista Brasileira de Geociências 30(2):222-225.

Neder, R.D., Leite, J.A.D., Figueiredo, B.R., McNaughton, N.J., 2002. 1.76 Ga volcanoplutonism in the southwestern Amazonian craton, Aripuanã-MT, Brazil: tectono-stratigraphic implications from SHRIMP U-Pb zircon data and rock geochemistry. Precambrian Research 119, 171-187.

Parrish, R.R.; 1990. U-Pb dating of monazite and its application to geological problems. Canadian Journal of Earth Sciences, 27, 1431-1450.

Paterson, S. R. \& Ducea, M. N.; 2015. Arc Magmatic Tempos: Gathering the Evidence. Elements, 11, 91-98.

Pinho M.A.S.; Chemale Jr. F.; Schmus W.R.V.; Pinho F.E.C. 2003. U-Pb and Sm-Nd evidence for 1.76-1.77 Ga magmatism in the Moriru region, Mato Grosso, Brazil: implications for province boundaries in the SW Amazonian Cráton. Prec. Res., 126: 125.

Poitrasson F.; Chenery S.; Bland D.J.; 1996. Contrasted monazite hydrothermal alteration mechanisms and their geochemical implications. Earth and Plan. Sc. Lett., 145:79-96.

Poitrasson F.; Chenery S.; Shepherd T.J.; 2000. Electron microprobe and LA-ICP-MS study of monazite hydrothermal alteration: implications for U-Th-Pb geochronology and nuclear ceramics. Geoch. Cosmoch. Acta, 64:3283-3297. 
Rasmussen, B.; Fletcher, I.R.; McNaughton, N.J.; 2001. Dating lowgrade metamorphism by SHRIMP U-Pb analysis of monazite in shales: Geology, v. 29, p. 963-966.

Rasmussen, B.; Sheppard, S.; Fletcher, I.R.; 2006. Testing ore deposit models using in situ $\mathrm{U}-\mathrm{Pb}$ geochronology of hydrothermal monazite: Paleoproterozoic gold mineralization in northern Australia. Geology; v. 34; no. 2; p. 77-80.

Santos, J.O.S. 2000. Os terrenos Paleoproterozóicos da Província do Tapajós e as mineralizações de ouro associadas. Tese de Doutorado, Universidade Federal do Rio Grande do Sul, Porto Alegre, v.1, 208p.

Santos J.O.S. 2003. Geotectônica dos escudos das Guianas e Brasil- Central. In: Bizzi L.A., Schobbenhaus C., Vidotti R.M., Gonçalves J.H. (eds). Geologia, tectônica e recursos minerais do Brasil. CPRM. Brasília. CD-ROM.

Santos J.O.S.; Van Breemen O.B.; Groves D.I.; Hartmann L.A.; Almeida M.E.; McNaughton N.J.; Fletcher I.R.; 2004. Timing and evolution of multiple Paleoproterozoic magmatic arcs in the Tapajós Domain, Amazon Craton: constraints from SHRIMP and $\mathrm{TIM}_{\mathrm{S}}$ zircon, baddeleyite and titanite U-Pb geochronology. Precambrian Research, 131:73-109.

Serrato, A.A.A.; 2014. Geocronologia e evolução do sistema hidrotermal do depósito aurífero de Juruena, Província Aurífera de Alta Floresta (MT), Brasil. Dissertação de mestrado, Unicamp.

Schulz, B. \& Von, Raumer, J. F. 2011. Discovery of Ordovician-Silurian metamorphic monazite in garnet metapelites of the Alpine External Aiguilles Rouges Massif. Swiss J Geosciences., 104:67-79.

Silva M.G. \& Abram M.B. 2008. Projeto Metalogenia da Província Aurífera JuruenaTelesPires, Mato Grosso. Goiânia, Serviço Geológico Brasileiro, CPRM, 212 p.

Smith, J.B.; Barley, M.E.; Groves, D.I.; Krapez, B.; McNaughton, N.J.; Bickle, M.J.; and Chapman, H.J.; 1998. The Scholl shear zone, West Pilbara: Evidence for a domain boundary structure from integrated tectonostratigraphic analyses, SHRIMP U-Pb dating and isotopic and geochemical data of granitoids: Precambrian Research, v. 88, p. 143171.

Souza J.P.; Frasca A.A.S.; Oliveira C.C.; 2005. Geologia e Recursos Minerais da Província Mineral de Alta Floresta. Relatório Integrado. Brasília, Serviço Geológico Brasileiro, CPRM,164 p.

Souza, V. S.; Teixeira, L. M.; Botelho, N. F.; 2005. Datação U-Th-Pb de Monazita Hidrotermal e sua aplicação na Geocronologia da Mineralização de Estanho em zonas de greisen do sistema Granítico Palanqueta, Depósito do Bom Futuro (RO), Revista Brasileira de Geociências, v. 35.

Tassinari, C.C.G. \& Macambira, M.J.B. 1999. Geological provinces of the Amazonian Craton. Episodes, v. 22, p. 173-182.

Tassinari C.C.G. \& Macambira M.J.B. 2004. A evolução tectônica do Cráto Amazônico. In: Matasso-Neto V., Bartorelli A., Carneiro CDR, Brito-Neves B.B. (eds.) Geologia do continente sul-americano: Evolução da obra de Fernando Flávio Marques de Almeida. Beca, São Paulo, 673p. 
Teixeira, W.; Tassinari, C.; Cordani, U.; Kawashita, K.; 1989. A review of the geochronology of the Amazonian Cráton: Tectonic implications. Precambrian Research, v. 42, n.3/4, p. 213-227.

Teufel S. \& Heinrich W. 1997. Partial resetting of the U-Pb isotope system in monazite through hydrothermal experiments: an SEM and U-Pb isotope study. Chem. Geol., 137:57-74.

Williams, I.S.; Claesson, S.; 1987. Isotopic evidence for the Precambrian provenance and Caledonian metamorphism of high grade paragneisses from the Steve Nappes, Scandanavian Caledonides, II. Ion microprobe zircon U-Th- $\mathrm{Pb}$ : Contributions to Mineralogy and Petrology, v. 97, p. 205-217. 


\section{$\underline{\text { ANEXOS }}$}




\section{ANEXO I - Comprovante de submissão de artigo à revista Journal of South}

\section{American Earth Sciences.}

Submission Confirmation faridchemale@gmail.com Gmail

kellogg@geol.sc.edu

Mover para a Caixa de Entrada

Submission Confirmation Entrada $x$

Journal of South American Earth Sciences por eesmail.elsevier.com

para mim, faridcj

Research Paper

Dear Farid,

We have received your article "ISOTOPE GEOLOGY OF THE GRANITIC ROCKS FROM TRAIRÃO REGION ALTA FLORESTA GOLD PROVINCE SOUTHERN AMAZONIAN CRATON" for consideration for publication in Journal of South American Earth Sciences.

Your manuscript will be given a reference number once an editor has been assigned.

To track the status of your paper, please do the following

1. Go to this URL: http://ees.elsevier.com/sames/

2. Enter these login details:

Your username is: faridchemale@gmail.com

If you need to retrieve password details, please go to:

http://ees.elsevier.com/sames/automail query.asp

3. Click [Author Login]

This takes you to the Author Main Menu.

4. Click [Submissions Being Processed]

Thank you for submitting your work to this journal.

Kind regards,

Elsevier Editorial System

Journal of South American Earth Sciences

Please note that the editorial process varies considerably from journal to journal. To view a sample editorial process, please click here: http://help.elsevier.com/app/answers/detail/p/7923/a id/160

For further assistance, please visit our customer support site at http://help.elsevier.com/app/

for solutions on a range of topics, find answers to frequently asked questions and learn more about EES via interactive tutorials. You will also find our $24 / 7$ support contact details should you need any further assistance from one of our customer support representatives. 


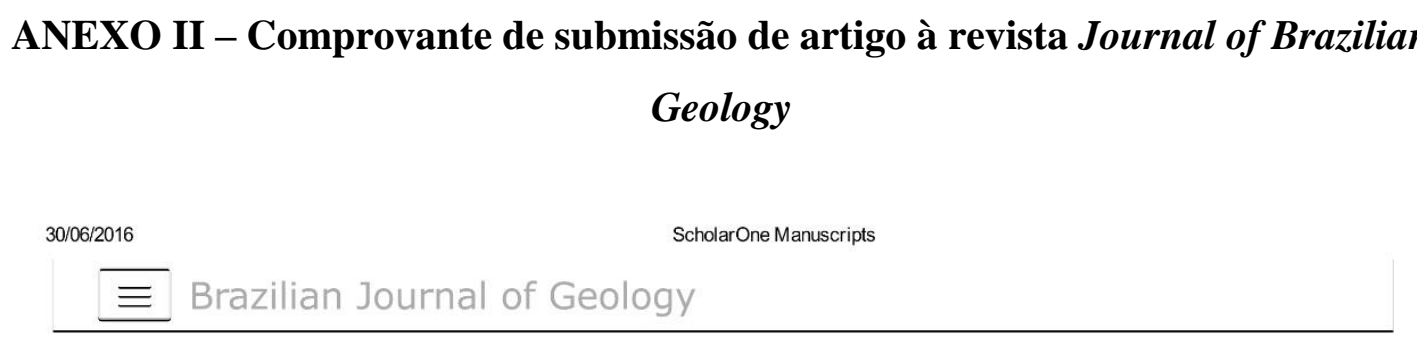

\section{Submission Confirmation}

Thank you for your submission

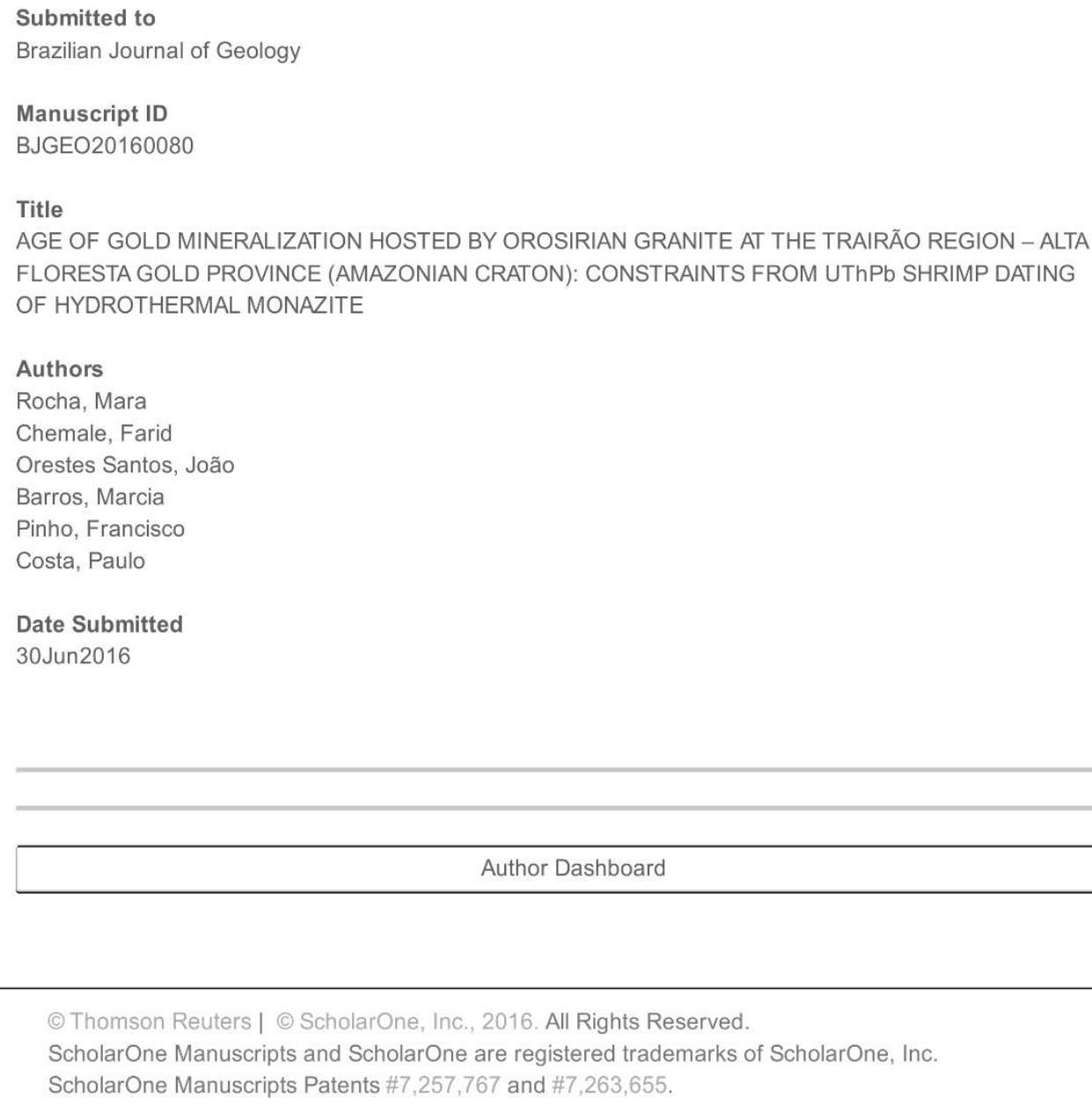




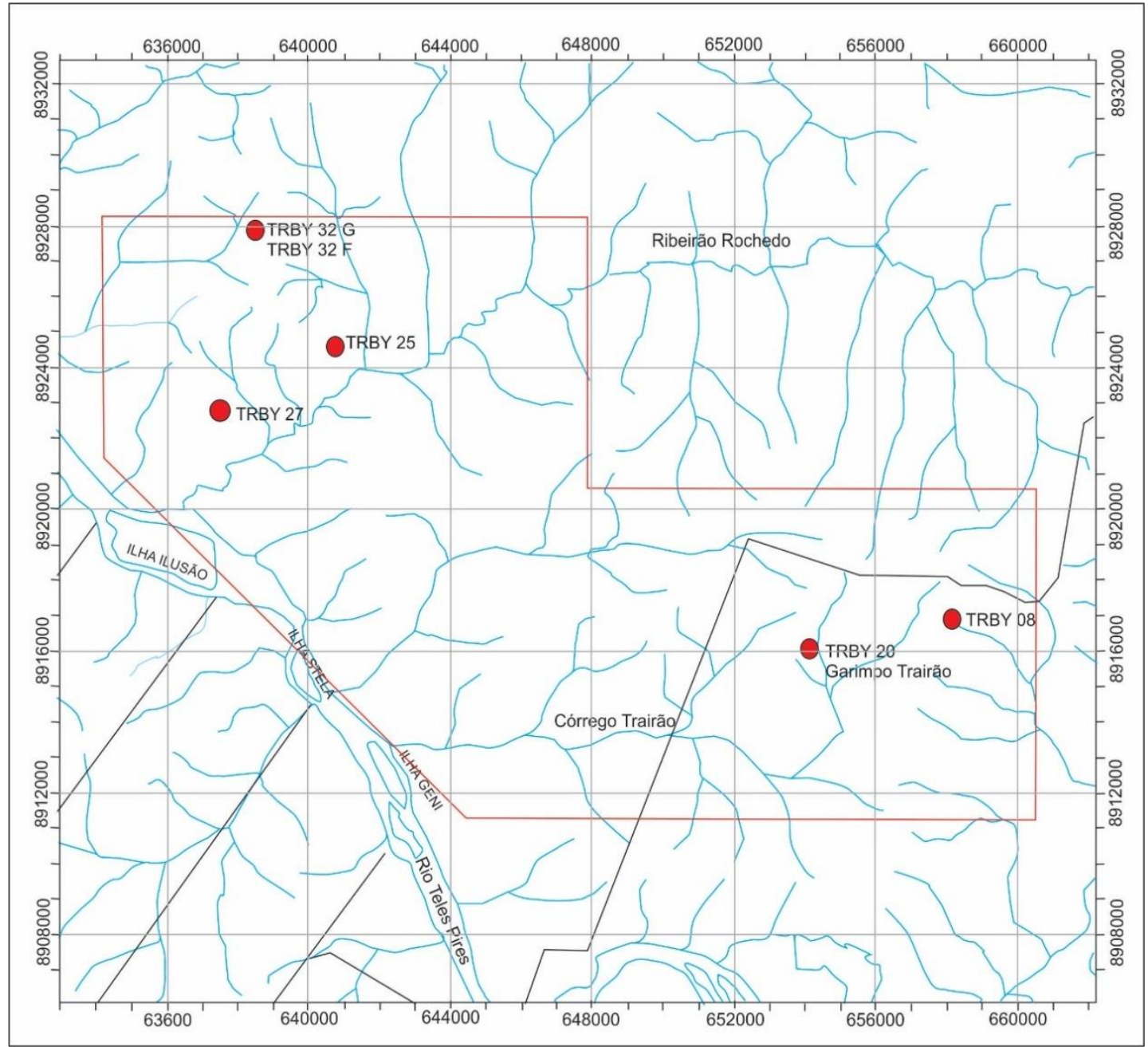

ANEXO III - Mapa de Localização das amostras datadas. 


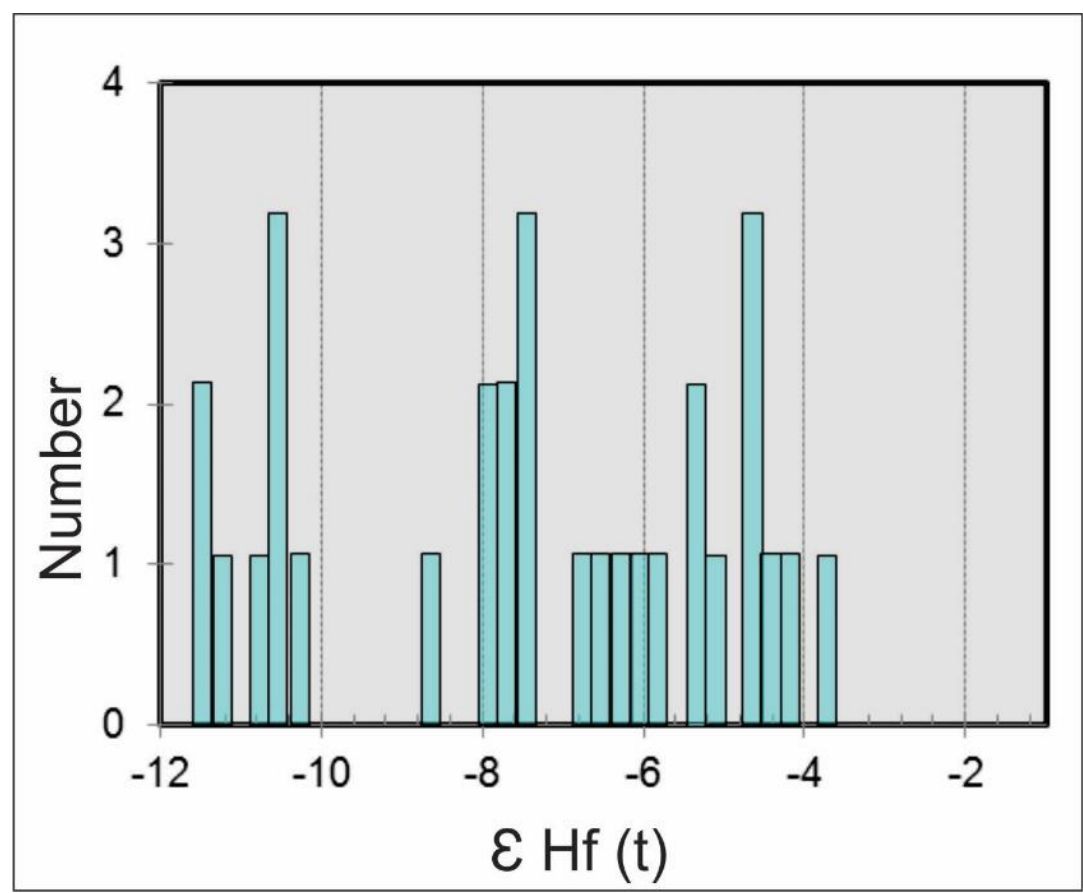

ANEXO IV - Diagrama de densidade de probabilidade para $\mathcal{E}$ Hf (T) em zircão do Suíte Intrusiva Nhandu e da Suíte Intrusiva Matupá.

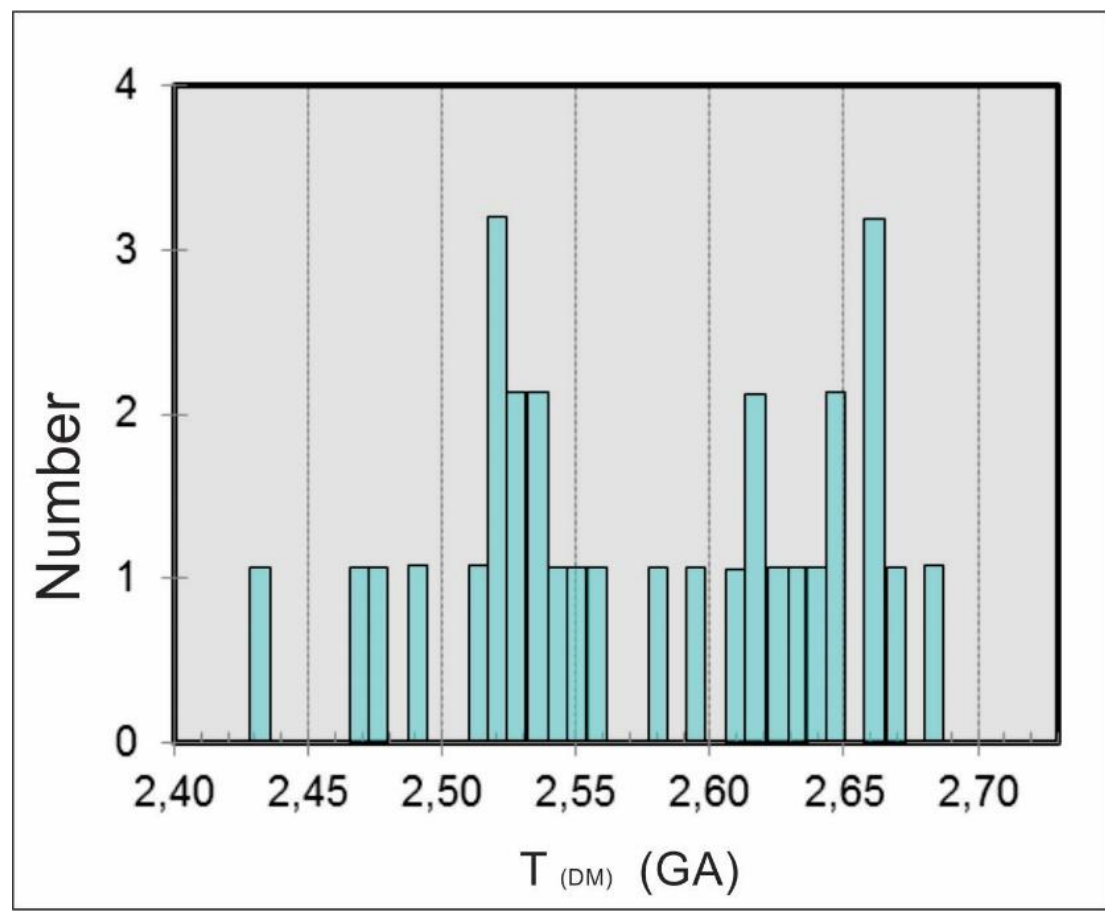

ANEXO V - Diagrama de densidade de probabilidade para $\mathrm{T}_{\mathrm{DM}}(\mathrm{Ma})$ em zircão da Suíte Intrusiva Nhandu e da Suíte Intrusiva Matupá. 